Universidade de São Paulo

Instituto de Astronomia, Geofísica e Ciências Atmosféricas

Departamento de Astronomia

Gleidson Gomes da Silva

\title{
Ressonâncias de Três Corpos: Estudo da Dinâmica da Zona Habitável do Sistema Exoplanetário GJ581
}

São Paulo 

Gleidson Gomes da Silva

\section{Ressonâncias de Três Corpos: Estudo da Dinâmica da Zona Habitável do Sistema Exoplanetário GJ581}

Tese apresentada ao Departamento de Astronomia do Instituto de Astronomia, Geofísica e Ciências Atmosféricas da Universidade de São Paulo como requisito parcial para a obtenção do título de Doutor em Ciências.

Área de Concentração: Astronomia Orientador: Prof. Dr. Sylvio Ferraz-Mello

Versão Corrigida. O original encontra-se disponível na Unidade

São Paulo 

Ao meu filho e minha mãe, Henri e Josefina! Aos meus tios, Heleno Filho e José(Duda)! À minha tia Fátima e aos meus primos Fabrício e Flávio(em memória)! 



\section{Agradecimentos}

À Deus, pela sua presença constante em minha vida.

Ao Prof. Sylvio Ferraz-Mello, pela orientação, apoio e confiança na realização deste trabalho.

À Prof. ${ }^{a}$ Tatiana Michtchenko, pelo apoio e sugestões para o trabalho.

À toda minha família, em particular aos meus irmãos, Cêça e Gleibson, pelo incentivo.

À minha mãe, pelo apoio incondicional.

À minha madrinha Lêda e família, pelo apoio e carinho.

Aos meus tios e tias: Zezé, Birino, Marivaldo, Maria, Leonor, Carminha, pelo incentivo.

Aos amigos: Hércules Lima, Mázio Ribeiro, Marcos Vinícius, Fernando Luna, George Kouzo, Airton Castro, Cláudio Teles, pelo incentivo e apoio desde do início.

Aos amigos: Cícero Alfredo, Adson Mota, Ricardo Adriano(Rico), Marcos Paulo, Roberto, Severino Horácio, Gedeon, Carlos, pelo incentivo e amizade.

Aos amigos: Maximiliano, Fabiana, Sandra(Sukita), Admilson, Wuilson e Wilson(Wa) pelo incentivo e amizade.

Aos amigos: Alan Alves, Eduardo Andrade, Adrian Colucci, pelo apoio e discussões.

Aos amigos: Ivan Rocha, Ademar Ferreira, pelo companheirismo, amizade e compreensão.

Ao Enzo Djo, pelo carinho e sorrisos de criança que muito me alegraram nos momentos difíceis.

Aos amigos: Fernando Cachucho, Raul Puebla, Marcos Tadeu pela amizade, incentivo, paciência, apoio e discussões.

Às amigas: Jane Roberta e Marina Freitas pelo carinho, atenção, incentivo, companheirismo e paciência. 
Às amigas: Filomena Nheiona, Priscila Ramos, Márcia Ribeiro, Joice Lima, Aparecida Mendes, Conceição Sano, Regina Iacovelli, pelo incentivo e amizade.

À equipe de informática: Luis Manrique, Marcos Santos, Carlos Paladino, Ulisses Manzo, pelo apoio técnico e amizade.

Aos amigos: Paulo Jeferson, Fernanda Nogueira, Nahin, pela amizade e brincadeiras.

À CAPES, pelo apoio financeiro;

Ao Instituto de Astronomia, Geofísica e Ciências Atmosféricas-IAG/USP por ter me dado todas as condições necessárias para a realização deste trabalho.

Esta tese foi escrita em LATEX com a classe IAGTESE, para teses e dissertações do IAG. 
"Quando a noite aparece, é que os olhos dos homens conseguem divisar o esplendor das estrelas."

Chico Xavier( Emmanuel ) 



\section{Resumo}

Este trabalho considera a existência de ressonâncias de três corpos na zona habitável(ZH), da estrela GJ 581 (Gliese 581), envolvendo os planetas GJ581 c, GJ581 d e um terceiro planeta dentro da ZH. Inicialmente, descreve-se o trabalho de desenvolvimento da função perturbadora devida à atração dos dois planetas considerados sobre o planeta teste. Como as perturbações mútuas envolvem apenas dois corpos (perturbado e perturbador), a obtenção do Hamiltoniano médio com termos que envolvem as longitudes médias de três corpos diferentes, exige o cálculo efetivo das perturbações de primeira ordem. Isso foi feito usando a teoria das séries de Lie, e o Hamiltoniano médio é aquele resultante de uma aplicação da teoria de perturbações, interrompida após a obtenção da média desejada(de segunda ordem nas massas). Em seguida apresentamos algumas ressonâncias de três corpos obtidas com a teoria de Lie e seus coeficientes e fazemos uma revisão de alguns conceitos básicos relativos à teoria da difusão lenta em sistemas Hamiltonianos multidimensionais sob a ação de pequenas perturbações, em particular, a teoria da difusão lenta desenvolvida por Chirikov (1979). A teoria de Chirikov foi usada para gerar um novo sistema de variáveis canônicas em que os momentos se orientam ao longo e através da ressonância. As soluções do Hamiltoniano transformado foram simuladas com a utilização do mapa simplético de Hadjidemteriou e a difusão das soluções foi estimada usando-se a fórmula usual introduzida por Chirikov. Os resultados estão mostrados no final deste trabalho. Estimamos os valores possíveis de excentricidades das órbitas dos planetas GJ581c e GJ581d, de modo a possibilitar a existência de um outro planeta em ZH. Optou-se por usar o mapa de Hadjidemetriou pois ele permite simulações muito mais rápidas do que os integradores, e assim permite a simulação das soluções em um número maior de pontos e também a consideração de diversos sistemas de massas. 



\section{Abstract}

This work considers the existence of three-body resonances in the habitable zone (HZ), of the star GJ 581(Gliese 581), involving the planets GJ581 c, GJ581 d and a third planet inside the HZ. Initially, we describe the work of the development of the disturbing function due to the attraction of the two planets considered on the test planet. As the mutual perturbations involve only two bodies (perturbed and disturbing), the obtention of the mean Hamiltonian with terms involving the three means longitudes of three different bodies, requires the calculation of the perturbations of the first order. This was done using the theory of the Lie's series, and the average Hamiltonian is that resulting from a application of the perturbations theory, stopped after the obtention of the desired average (second order in the masses). Then we present some three bodies resonances obtained with the theory of Lie and their coefficients. We review some basic concepts related to the theory of slow diffusion in the multidimensional Hamiltonian systems under action of small perturbations, in particular, the theory of slow diffusion developed by Chirikov (1979). The Chirikov's theory was used to generate a new system of canonical variables where the moments are oriented along and across the resonance. The solutions of the transformed Hamiltonian were simulated using the Hadjidemetriou's sympletic map and the diffusion of solutions was estimated using the usual formula introduced by Chrikov. The results are show at the end of this work. We estimate the possible values of the eccentricities of the orbits of planets G581 c and GJ581 d, to permit the existence of another planet inside HZ. We chose to use the Hadjidemetriou's map because it allows faster simulations than integrators, and thus the simulations of solutions for a higher number of points and considering a range of mass systems as well. 



\section{Lista de Figuras}

2.1 Vetores posição, $\mathbf{r}$ e $\mathbf{r}^{\prime}$ das massas $m$ e $m^{\prime}$ em relação a massa central $M_{c} \quad$. 26

2.2 Localização do planeta GJ581g . . . . . . . . . . . . . . . . . 34

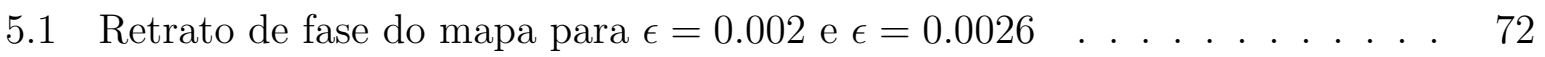

5.2 Passeio aleatório com barreira . . . . . . . . . . . . . . . . . 80

5.3 Evolução de $S(t)$ e $\bar{S}(n T)$ para 100 órbitas com $\epsilon=0.007 \ldots$. . . . . . . . 88

7.1 Superfícies ressonantes nos espaço das ações $\left(P_{1}, P_{2}, P_{3}\right) \ldots \ldots \ldots$

7.2 Superfícies ressonantes nos espaço das excentricidades $\left(e_{1}, e_{2}, e_{3}\right) \ldots \ldots$. . 110

7.3 Difusão em semieixo para $t=10^{4}$ anos. $m_{1}=m_{3}=5 M_{\otimes} \ldots \ldots$. . . . 113

7.4 Difusão em semieixo para $t=10^{5}$ anos. $m_{1}=m_{3}=5 M_{\otimes} \ldots \ldots \ldots \ldots$

7.5 Difusão em excentricidade para $t=10^{4}$ anos. $m_{1}=m_{3}=5 M_{\otimes} . \ldots . .113$

7.6 Difusão em excentricidade para $t=10^{5}$ anos. $m_{1}=m_{3}=5 M_{\otimes}$. . . . . . 113

7.7 Separatrizes paras as massas $m_{1}=m_{3}=5 M_{\otimes} \ldots \ldots \ldots \ldots \ldots$. . . . . 114

7.8 Difusão em semieixo para $t=10^{3} \cdot m_{1}=5, m_{3}=6 M_{\otimes} \ldots \ldots \ldots \ldots$

7.9 Difusão em semieixo para $t=10^{4} . m_{1}=5, m_{3}=6 M_{\otimes} \ldots \ldots \ldots \ldots$

7.10 Difusão em excentricidade para $t=10^{3} \cdot m_{1}=5 M_{\otimes}, m_{3}=6 M_{\otimes} . \ldots . . .115$

7.11 Difusão em excentricidade para $t=10^{4}$ anos. $m_{1}=5 M_{\otimes}, m_{3}=6 M_{\otimes} . \quad$. . 115

7.12 Separatrizes para as massas $m_{1}=5 M_{\otimes}, m_{3}=6 M_{\otimes} \ldots \ldots \ldots \ldots$

7.13 Difusão em semieixo para $t=10^{3}$ anos. $m_{1}=5 M_{\otimes}, m_{3}=4 M_{\otimes}$. . . . . 116

7.14 Difusão em semieixo para $t=10^{4}$ anos. $m_{1}=5 M_{\otimes}, m_{3}=4 M_{\otimes} \ldots \ldots$. . 116

7.15 Difusão em excentricidade para $t=10^{3}$ anos. $m_{1}=5 M_{\otimes}, m_{3}=4 M_{\otimes} . \ldots 116$

7.16 Difusão em excentricidade para $t=10^{4}$ anos. $m_{1}=5 M_{\otimes}, m_{3}=4 M_{\otimes} . \cdots 116$

7.17 Separatrizes para as massas $m_{1}=5 M_{\otimes}, m_{3}=4 M_{\otimes} \ldots \ldots \ldots$ 
A.1 Superfície de energia separadas em regiões isoladas . . . . . . . . . . . . 132

A.2 Superfície de energia separadas em regiões não isoladas . . . . . . . . . . 133

A.3 Teia de Arnold para um Hamiltoniano de uma partícula livre. . . . . . . . 133

A.4 Difusão ao longo e através da camada ressonante . . . . . . . . . . . . . . 134 


\section{Lista de Tabelas}

2.1 Parte secular para os planetas GJ581d e teste . . . . . . . . . . . . . 34

2.2 Parte direta para os planetas GJ581d e teste . . . . . . . . . . . 35

2.3 Parte indireta para os planetas GJ581d e teste . . . . . . . . . . . 35

2.4 Parte Indireta para os planetas GJ581c e teste . . . . . . . . . . . . . 36

2.5 Parte Direta para os planetas GJ581c e teste . . . . . . . . . . . . 36

2.6 Parte Indiretaireta para os planetas GJ581c e teste . . . . . . . . . . . 37

2.7 Parte Secular da Função perturbadora para os planetas GJ581d e GJ581c. 37

2.8 Parte Direta da Função perturbadora para os planetas GJ581d e GJ581c. 37

2.9 Parte Indireta da Função perturbadora para os planetas GJ581d e GJ581c. 37

4.1 Principais ressonâncias no intervalo considerado. . . . . . . . . . . 56

4.2 Multipletos da ressonância $\lambda_{1}-2 \lambda_{2}-2 \lambda_{3} \ldots \ldots \ldots \ldots$

4.3 Mulipletos para $e_{1}=0.05, e_{3}=0.05 \ldots \ldots \ldots \ldots$

5.1 Caminho aleatório com unidade de um passo igual $l \ldots \ldots$. . . . . . 79 



\section{Sumário}

1. Introdução . . . . . . . . . . . . . . . . . . . . . . . . . . . . . . . . 21

2. Análise de Ressonâncias . . . . . . . . . . . . . . . . . . . . . . . . . . . . 25

2.1 Importância das Ressonâncias No Estudo da Dinâmica de Corpos Celestes 25

2.2 Estudo de Ressonâncias . . . . . . . . . . . . . . . . . . . . . . 26

2.3 Ressonâncias de Três Corpos . . . . . . . . . . . . . . . . . . . . . . . 27

2.4 Expansão da Função Perturbadora . . . . . . . . . . . . . . . . . . . . . . 28

2.5 Uso da Função Perturbadora . . . . . . . . . . . . . . . . . . . . . . . . . . 32

2.6 Ressonâncias no sistema GJ581 . . . . . . . . . . . . . . . . . . . . . . . . 33

2.6.1 Ressonância 2/1 entre o planeta GJ581d e o planeta teste . . . . . . 34

2.6.2 Ressonância 3/1 Entre o Planeta GJ581c e o Planeta Teste . . . . . 36

2.6.3 Ressonância 5/1 Entre os Planetas GJ581d e GJ581c . . . . . . . 36

2.7 Variáveis de Delaunay e Poincaré . . . . . . . . . . . . . . . . . . . . 38

2.7.1 Variáveis de Delaunay . . . . . . . . . . . . . . . . . 38

2.7.2 Variáveis de Poincaré . . . . . . . . . . . . . . . . . . . 38

2.8 Conexão entre as variáveis de Poincaré e os elementos elípticos clássicos . . 39

2.9 Forma Hamiltoniana Para o Sistema Perturbado . . . . . . . . . . . . . . . 40

3. Hamiltonianos Médios Com Ressonâncias de Três Corpos . . . . . . . . . . . . . 47

3.1 Operador de Lie . . . . . . . . . . . . . . . . . . . . . . . . 47

3.2 Séries de Lie . . . . . . . . . . . . . . . . . . . . . . . . . . . . . . 48

3.3 Teoria de Perturbações com Séries de Lie . . . . . . . . . . . . . . . . . . 49

3.4 A equação Homológica . . . . . . . . . . . . . . . . . . . . . . . . . 50 
3.4.1 Regra da Média . . . . . . . . . . . . . . . 51

3.5 Solução da Equação Homológica . . . . . . . . . . . . . . . . . . . . . . . . 51

3.6 Cálculo do Hamiltoniano Médio Na Vizinhança de Ressonâncias de Três Corpos 52 3.6.1 Origem dos Termos que Envolvem Três Longitudes . . . . . . . . . 53

4. Resultados da Aplicação da Teoria de Lie ao Sistema GJ581 . . . . . . . . . . . 55

4.1 Ressonâncias Obtidas Utilizando a Teoria de Lie . . . . . . . . . . . . . . . 55

4.1.1 Coeficientes dos Multipletos da Ressonância $\lambda_{1}-2 \lambda_{2}-2 \lambda_{3} \ldots \ldots . \quad 55$

5. Alguns Tópicos da Teoria da Difusão . . . . . . . . . . . . . . . . . . . 71

5.1 Conceitos básicos . . . . . . . . . . . . . . . . . . 71

5.1.1 Aspecto Geométrico da Difusão . . . . . . . . . . . . . . . . . . . 71

5.1 .2 Overlaps De Ressonâncias . . . . . . . . . . . . . . . . 72

5.1 .3 Difusão na Astronomia . . . . . . . . . . . . . . . . . . . . 73

5.2 Difusão em um Passeio Aleatório . . . . . . . . . . . . . . . . . 73

5.2.1 Variáveis Aleatórias . . . . . . . . . . . . . . . . 73

5.2.2 Funções de Probabilidade . . . . . . . . . . . . . . . . . 74

5.2 .3 Passeio Aleatório . . . . . . . . . . . . . . . . 76

5.2 .4 Passeio aleatório com Barreiras _. . . . . . . . . . . . . 78

5.2.5 Barreira Refletora em $m=m_{1} \ldots \ldots \ldots$. . . . . . . 78

5.2.6 Barreira Absorvente em $m=m_{1} \ldots \ldots \ldots$. . . . . . 81

5.3 Equação de Fokker- Planck . . . . . . . . . . . . . . . . . . . . . 83

5.3.1 Equação de Fokker-Planck Para Um Sistema Hamiltoniano . . . . . 84

5.4 Cálculo do Coeficiente de Difusão . . . . . . . . . . . . . . . . . . 86

5.4.1 Coeficiente Segundo Froeschlé . . . . . . . . . . . . 86

5.4 .2 Coeficiente de Difusão . . . . . . . . . . . . . . . . . . 87

5.5 Taxa de Difusão Segundo Chirikov . . . . . . . . . . . . . . . . 87

6. Teoria da difusão de Chirikov . . . . . . . . . . . . . . . . . . . . . . . . . . 89

6.1 Geometria da ressonância guia no espaço das ações . . . . . . . . . . . . . 90

6.2 Mudança de base local . . . . . . . . . . . . . . . . . . . . . . . . 91

6.3 Hamiltoniano Nas Novas Ações . . . . . . . . . . . . . . . . . . . 93

6.4 Perturbação . . . . . . . . . . . . . . . . . . . . 95 
6.5 Estimativa da Variação Total das Componentes do momento p . . . . . . . 96 6.5.1 Equação da Separatriz . . . . . . . . . . . . . . . . . . . . . 97

6.6 A taxa de difusão . . . . . . . . . . . . . . . . . . . . . . . . . . . . . 99

6.6.1 Tensor de Difusão . . . . . . . . . . . . . . . . . . . . . . . 100

6.7 A aproximação de Estocasticidade Reduzida . . . . . . . . . . . . . . . . . 101

7. Aplicação da Teoria de Chirikov e Modelos Numéricos . . . . . . . . . . . . . . 103

7.1 Aplicação da Teoria de Chirikov ao Sistema GJ581 . . . . . . . . . . . . 103

7.2 Modelos Numéricos . . . . . . . . . . . . . . . . . . 105

7.2.1 O Mapa Simplético de Hadjidemetriou . . . . . . . . . . . . . . . 105

7.2 .2 Modelo Para Seis Ressonâncias . . . . . . . . . . . . . . . . . . . 107

7.3 Parâmetros Numéricos . . . . . . . . . . . . . . . . . . . 108

7.4 Mapas de Contorno . . . . . . . . . . . . . . . . . . . . . 110

7.4.1 Massas $m_{1}=m_{3}=5$ Massas da Terra . . . . . . . . . . . . . 110

7.4.2 Massas $m_{1}=5$ e $m_{3}=6$ Massas da Terra . . . . . . . . . . . 111

7.4.3 Massa $m_{1}=5$ e $m_{3}=4$ Massas da Terra . . . . . . . . . . . . . 112

8. Conclusões . . . . . . . . . . . . . . . . . . . . . . . . . . . . . . 119

Apêndice $\quad 121$

A. Resultados Básicos de Sistemas Dinâmicos . . . . . . . . . . . . . . . . . . . . . 123

A.1 Sistemas Integráveis . . . . . . . . . . . . . . . . . . . . . . . 123

A.2 Convexidade de $H_{0} \ldots \ldots \ldots \ldots \ldots \ldots \ldots$. . . . . . . . . . . . . . 124

A.3 Sistema Instável a Priori . . . . . . . . . . . . . . . . . . . . . . . 127

A.4 Sistemas Livres: Caminho de Difusão . . . . . . . . . . . . . . . . . . . . . 131

A.5 Difusão de Arnold . . . . . . . . . . . . . . . . . . . . . . . 132

A.6 Sistemas Quase Integráveis e Estabilidade . . . . . . . . . . . . . . . . . 134

A.7 A Conservação da Energia . . . . . . . . . . . . . . . . . . . . . . . . 137

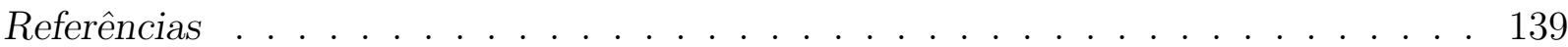



Capítulo 1

\section{Introdução}

Nos últimos anos tem crescido o número de planetas extrassolares descobertos. Claramente isso se deve ao aprimoramento das técnicas de deteç̧ão. Hoje são conhecidos 843 planetas dentre os quais existem 126 sistemas de múltiplos planetas ${ }^{1}$. Neste cenário, em 2007, um sistema ganhou bastante atenção dos Astrônomos: o sistema de planetas múltiplos orbitando a estrela Gliese 581. A estrela Gliese 581 é uma estrela anã vermelha que possui tipo espectral M3V localizada a aproximadamente 20,3 anos-luz da Terra, na constelação de Libra. Possui uma massa de aproximadamente um terço da massa solar. A atenção voltada para este sistema, foi devida ao fato da estrela ser orbitada pelo primeiro planeta potencialmente habitável fora do Sistema Solar, a Super-Terra GJ581c (para maiores detalhes ver S. Udry et al. (2007)). Além disso, em 2009 foi descoberto um dos menores exoplanetas conhecidos até agora, o planeta GJ581e (ver Mayor et al. (2009)). Todas estas descobertas foram feitas usando o espectrógrafo HARPS. Em 2010, Vogt et al. (2010) , analisando dados do HARPS e também do Keck Planet Search, anunciaram a descoberta de dois novos planetas orbitando a estrela GJ581, os planetas GJ581f e GJ581g. O planeta GJ581g é importante pois ele está situado no "meio" da $Z H$ desta estrela. Contudo, a existência destes dois planetas ainda é objeto de várias discussões. Em 2011, Gregory (ver, Gregory (2011)) fez uma análise estatística dos dados obtido por Vogt et al. (2010) e Mayor et al. (2009) e não pode confirmar a existência ou não de tais planetas. Em 2012, Tadeu dos Santos et al. (2012), concluiu que ainda não se tem observações suficientes que nos permitam afirmar com certeza a existência do planeta GJ581g. Como podemos observar o Sistema GJ581 representa um rico objeto de pesquisa em Astronomia.

\footnotetext{
${ }^{1}$ http://exoplanet.eu/catalog/
} 
Do ponto de vista dinâmico a Zona Habitável da estrela GJ581 é muito rica, devido a um grande número de ressonâncias de movimentos médios de três e dois corpos existentes entre os planetas GJ581c, GJ581d localizados na borda interior(0.10 AU) e exterior(0.18 AU) da $Z H$, respectivamente, e um planeta teste situado entre eles. Sabemos que as ressonâncias de três corpos são muito mais densas no espaço de fase do que as ressonâncias ordinárias. Portanto, estamos interessados em estudar a dinâmica da Zona Habitável influenciada por uma dada ressonância de três corpos, em particular a ressonância $\lambda_{1}-2 \lambda_{2}-2 \lambda_{3}$, onde $\lambda_{1}$, $\lambda_{2}$ e $\lambda_{3}$ são as longitudes médias dos planetas GJ581c, teste e GJ581d, respectivamente. Esta ressonância foi escolhida por estar relativamente isolada de outras ressonâncias.

Quando se fala em dinâmica planetária surgem naturalmente perguntas relativas à estabilidade e caos: Será a Zona Habitável da estrela Gliese 581 estável? , Existem processos difusivos dentro da Zona Habitável?,... Processos difusivos são estudados há muitos anos por vários pesquisadores, das mais diversas áreas. O primeiro processo difusivo a ser tratado foi a difusão de Arnold (Arnold (1964)). Na literatura existem uma infinidade de trabalhos que tratam da difusão lenta. Contudo, um dos mais importantes trabalhos é devido a Chirikov (1979). A formulação de Chirikov fornece uma maneira natural para conhecer a difusão em semieixo e excentricidade, e além disso fornece informações sobre a dinâmica dentro do domínio estocástico. As equações resultantes da aplicação da teoria de Chirikov foram feitas usando o mapa simplético de Hadjidemetriou (ver, Hadjidemetriou (1993), Ferraz-Mello (1996), Roig e Ferraz-Mello (1999)).

Esta tese é apresentada da seguinte forma:

No capítulo (2) desenvolvemos a função perturbadora para a interação gravitacional entre os três planetas, para em seguida construirmos a função Hamiltoniana.

Uma vez construído o Hamiltoniano, no capítulo (3) apresentamos, de forma breve, os principais conceitos da Teoria de Lie de perturbações. A aplicação da Teoria de Lie no Hamiltoniano obtido anteriormente nos permite obter os termos relativos às ressonâncias de três corpos. Vale notar que com este método encontra-se um grande número de ressonâncias de três corpos, a maioria delas, obviamente, fora da Zona Habitável. Selecionou-se aquelas que estão na ZH.

Dedicou-se o capítulo (4) à mostrar os resultados obtidos com a aplicação da teoria de Lie. Mostra-se, parcialmente, as expressões dos coeficientes dos multipletos relativos à 
ressonância $(1,-2,-2)$.

O capítulo (5) é dedicado a uma breve descrição de alguns tópicos relacionados à teoria difusão. Descrevemos o passeio aleatório que pode ser relacionado ao passeio aleatório dos elementos de um asteroide no espaço das excentricidades. De fato, o movimento caótico de um asteroide pode ser modelado como um passeio aleatório desde que a excentricidade, no problema de dois corpos, pode ser pensada como uma variável ação relacionada a ação $G$ de Delaunay (Varvoglis (2005)). Além disso, obtemos a equação de Fokker-Planck.

Dedicou-se o capítulo (6) à Teoria de Chirikov para estudar a difusão lenta em sistemas Hamiltonianos em muitas dimensões submetido a várias perturbações ressonantes (Chirikov (1979), Cincotta (2002)).

No capítulo (7) descrevemos os modelos numéricos usados na aplicação da Teoria de Chirikov e o mapa de Hadjidemetriou usado nos experimentos numéricos. Em seguida, discutem-se os resultados das simulações para difusão em semieixo e excentricidade.

Por último, o capítulo (8) é dedicado às considerações finais. 
Capítulo 2

\section{Análise de Ressonâncias}

\subsection{Importância das Ressonâncias No Estudo da Dinâmica de Corpos Celestes}

As ressonâncias orbitais desempenham um importante papel na compreensão de sistemas planetários. Ao longo dos anos, muitos trabalhos têm sido dedicados ao estudo de ressonâncias e suas implicações na dinâmica de tais sistemas (ver, Michtchenko e Ferraz-Mello (2001)). Em particular, o estudo de ressonâncias de movimentos médios e suas consequências para a evolução e estrutura do cinturão de asteroides (ver, Nesvorný e Morbidelli (1998b)) e dos sistemas extrasolares (ver, Michtchenko et al. (2006)). Sabemos que os sistemas exoplanetários diferem bastante do sistema solar no que diz respeito à sua estrutura.

Muitos trabalhos recentes tratam da migração planetária para sistemas exoplanetários em ressonâncias (ver, Beaugé et al. (2006), Ferraz-Mello et al. (2003)), ver também Hadjidemetriou e Voyatzis (2011). As ressonâncias de movimentos médios têm grande importância para difusão no cinturão de asteroides. De fato, sabemos que muitos asteroides inicialmente nas ressonâncias $(3: 1,4: 1)$ com Júpiter podem chegar à altas excentricidades em uma curta escala de tempo (ver, Ferraz-Mello e Klafke (1991), Klafke et al. (1992)). Estes trabalhos contribuem com o que foi dito por Wisdon em seu trabalho sobre a origem dos Kirkwood gaps na ressonância 3 : 1 com Júpiter (ver, Wisdom (1982)). Podemos ver que para ressonâncias próximas a Júpiter, espera-se que grande parte das órbitas sejam caóticas, paralelamente a isto, o valor da excentricidade necessária para cruzar a órbita de Júpiter é pequena $(e \approx 0.3)$. Sem um mecanismo que possa fornecer um "atalho"para altos valores de excentricidade, tais como as órbitas periódicas ressonantes, a maneira 
como um caminho aleatório pelo qual a excentricidade cresce é semelhante a um processo difusivo(ver,Varvoglis et al. (2001)). Durante muito tempo no estudo da evolução de asteroides negligenciou-se a importância das ressonâncias de movimentos médio de três corpos, um dos pioneiros trabalhos tratando a respeito de tais ressonâncias foi feito por Fernández e Beaugé em 1988 (ver, Fernandez e Beauge (1988)), para um asteroide, Júpiter e Saturno. Em 1988 Murray e colaboradores(ver, Murray et al. (1998)) ressaltaram a importância das ressonâncias de três corpos para o cinturão de asteroides e estimam, analiticamente, o tempo de Lyapunov e o tempo de difusão para algumas ressonâncias entre o asteroide, Júpiter e Saturno. Nesvorný e Morbidelli fornecem um modelo analítico para ressonâncias de movimentos médios de três corpos restrito(ver, Nesvorný e Morbidelli (1998b), Nesvorný e Morbidelli (1998a)). Em seu pioneiro trabalho, Cachucho e colaboradores (ver,Cachucho et al. (2010) e Cincotta (2002)) estudaram a difusão, aplicando a teoria de Chirikov (ver, Chirikov (1979) à ressonância $(5,-2,-2)$ da família de (490) Veritas.

\subsection{Estudo de Ressonâncias}

A análise de ressonâncias em um dado sistema é feita estudando-se o comportamento do ângulo ressonante correspondente usando as equações de Lagrange para estudar as variações dos elementos orbitais. Para obtermos as equações de Lagrange, usamos a Função Perturbadora $R$. O potencial perturbador $R$ descreve a perturbação de um corpo na órbita kepleriana ao redor de um outro corpo:

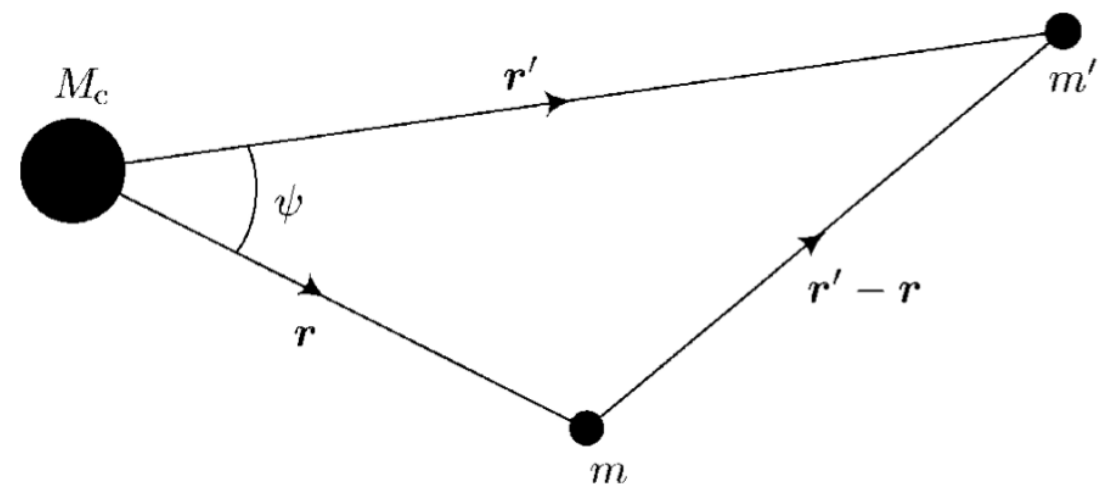

Figura 2.1: Vetores posição, $\mathbf{r}$ e $\mathbf{r}^{\prime}$ das massas $m$ e $m^{\prime}$ em relação a massa central $M_{c}$ 


$$
R=G m^{\prime}\left(\frac{1}{\Delta}-\frac{\mathbf{r} \cdot \mathbf{r}^{\prime}}{r^{\prime 3}}\right)
$$

onde $G$ é a constante gravitacional, $m^{\prime}$ é a massa do corpo perturbador, $\Delta$ é a distância entre os dois corpos, $\mathbf{r}$ é o raio vetor astrocêntrico do corpo perturbado, $\mathbf{r}^{\prime}$ o do corpo perturbador. O primeiro termo que aparece dentro dos parenteses em (2.1) representa o potencial no corpo perturbado devido à atração do perturbador. O segundo termo representa o efeito, no corpo perturbado, devido à força exercida pelo perturbador na estrela central.

Em geral, a função perturbadora é desenvolvida em séries de Fourier dos elementos orbitais, angulares, $\varpi, \Omega$ e $\lambda$, longitude do pericentro, longitude do nodo ascendente e longitude média, com coeficientes dependendo de $a, e, I$, semi-eixo maior, excentricidade e inclinação, tendo a seguinte forma geral:

$$
R=G m \sum S\left(a, a^{\prime}, e, e^{\prime}, I, I^{\prime}\right) \cos \Phi
$$

onde

$$
\Phi=j_{1} \lambda^{\prime}+j_{2} \lambda+j_{3} \varpi^{\prime}+j_{4} \varpi+j_{5} \Omega^{\prime}+j_{6} \Omega
$$

e a soma é feita sobre os números inteiros $j_{1}, j_{2}, j_{3}, j_{4}, j_{5}, j_{6}$. É importante notar que $S$ é também função destes inteiros. Além disso, temos as seguintes propriedades matemáticas, conhecidas como propriedades características de D' Alembert:

$$
\begin{aligned}
& \text { (i) } S \text { é da ordem de } e^{\left|j_{4}\right|} \cdot e^{\prime\left|j_{3}\right|} \cdot I^{\left|j_{5}\right|} \cdot I^{\left|j_{6}\right|} \\
& \text { (ii) } j_{5}+j_{6} \text { é par } \\
& \text { (iii) } j_{1}+j_{2}+j_{3}+j_{4}+j_{5}+j_{6}=0 \\
& \text { (iv) }\left|j_{1}+j_{2}\right| \leq\left|j_{3}\right|+\left|j_{4}\right|+\left|j_{5}\right|+\left|j_{6}\right|
\end{aligned}
$$

\subsection{Ressonâncias de Três Corpos}

Se observarmos a expansão da função perturbadora (2.2), notaremos que ela não apresenta harmônicos dependendo da combinação das longitudes médias de três corpos. Este fato poderia nos levar a concluir, erradamente, que as ressonâncias de três corpos não têm 
efeito, pois os harmônicos correspondentes não aparecem na função perturbadora. Contudo, ao aplicarmos a teoria de pertubações de Lie, vemos que tais harmônicos aparecem em segunda ordem nas massas, ao calcularmos os colchetes de Poisson.

Uma ressonância de três corpos corresponde a uma relação do tipo

$$
k_{1} \dot{\lambda}_{1}+k_{2} \dot{\lambda}_{2}+k_{3} \dot{\lambda}_{3} \approx 0
$$

onde $k_{1}, k_{2}, k_{3} \in \mathbb{Z}^{*}$ e $\dot{\lambda}_{1}, \dot{\lambda}_{2}, \dot{\lambda}_{3}$ são os movimentos médios de três corpos diferentes. O ângulo ressonante, correspondente a ressonância de três corpos, é da forma

$$
\sigma_{k_{4}, k_{5}, k_{6}, k_{7}, k_{8}, k_{9}}=k_{1} \lambda_{1}+k_{2} \lambda_{2}+k_{3} \lambda_{3}+k_{4} \varpi_{1}+k_{5} \varpi_{2}+k_{6} \varpi_{3}+k_{7} \Omega_{1}+k_{8} \Omega_{2}+k_{9} \Omega_{3}
$$

A origem e um estudo mais detalhado das ressonâncias de três corpos serão dados no capítulo (3).

\subsection{Expansão da Função Perturbadora}

De forma rigorosa para fazermos uso da função perturbadora é necessário que tenhamos todos os possíveis argumentos em sua expansão, contudo na prática utilizamos um número pequeno de argumentos, precisamente os termos de interesse (ver,Ellis e Murray (2000)). Da equação (2.1), podemos escrever a função perturbadora, para o perturbador externo, como

$$
R=\frac{\mu^{\prime}}{a^{\prime}}\left(R_{D}+\alpha R_{E}\right)
$$

e para o perturbador interno

$$
R^{\prime}=\frac{\mu}{a}\left(R_{D}+\frac{1}{\alpha^{2}} R_{I}\right)
$$

onde $\alpha=a / a^{\prime}$ é a razão entre o semieixo maior do corpo interno e corpo externo, $\mu^{\prime}=G m^{\prime}$ e $\mu=G m$. A parte direta é dada por

$$
R_{D}=\frac{a^{\prime}}{\Delta}
$$

$\operatorname{com} \Delta^{2}=r^{2}+r^{\prime 2}-2 r r^{\prime} \cos \psi$. Como $\mathbf{r} \cdot \mathbf{r}^{\prime}=r r^{\prime} \cos \psi$, onde $\psi$ é o ângulo entre os vetores posição, como na figura (2.1), tem-se que

$$
R_{E}=-\frac{r}{a} \frac{a^{\prime 2}}{r^{\prime 2}} \cos \psi
$$


e

$$
R_{I}=-\frac{r^{\prime}}{a^{\prime}} \frac{a^{2}}{r^{2}} \cos \psi
$$

Usando coeficientes de Laplace para expandir (2.7), temos

$$
\begin{aligned}
R_{D} & =\sum_{i=0}^{\infty} \frac{(2 i)}{i !} \frac{(-1)^{i}}{2^{2 i+1}} \alpha^{i} \\
& \times \sum_{j=-\infty}^{\infty}\left(\sum_{l=0}^{\infty} \frac{(-1)^{l}}{l !} \sum_{k=0}^{l}\left(\begin{array}{l}
l \\
k
\end{array}\right)(-1)^{k} \alpha^{l} \frac{d^{l}}{d \alpha^{l}} b_{i+1 / 2}^{(j)}(\alpha)\right) \\
& \times \sum_{s=0}^{i} \sum_{l=0}^{i-s} \frac{(-1)^{s} 2^{2 s}}{(i-s-l) ! l !} \sum_{n=0}^{[s / 2]} \frac{(2 s-4 n+1)(s-n) !}{2^{2 n} n !(2 s-2 n+1)} \\
& \times \sum_{m=0}^{s-2 n} \kappa_{m} \frac{(s-2 n-m) !}{(s-2 n+m) !} \sum_{p, p^{\prime}=0}^{s-2 n} F_{s-2 n, m, p}(I) F_{s-2 n, m, p^{\prime}}\left(I^{\prime}\right) \\
& \times \sum_{q, q^{\prime}=-\infty}^{+\infty} \chi_{i+j-2 l-2 n-2 p+q}^{i+k, i+j-2 l-2 n-2 p}(e) \chi_{i+j-2 l-2 n-2 p^{\prime}+q^{\prime}}^{-(i+k+1), i+j-2 l-2 n-2 p^{\prime}}\left(e^{\prime}\right) \\
& \times \cos ^{\prime}\left[\left(i+j-2 l-2 n-2 p^{\prime}+q^{\prime}\right) \lambda^{\prime}-(i+j-2 l-2 n-2 p+q) \lambda\right. \\
& \left.-q^{\prime} \varpi^{\prime}+q \varpi+\left(m-s+2 n+2 p^{\prime}\right) \Omega^{\prime}-(m-s+2 n+2 p) \Omega\right]
\end{aligned}
$$

onde $\kappa_{m}=1$ se $m=0$ e $\kappa_{m}=2$ se $m>0$, as séries $R_{E}$ e $R_{I}$ são dadas por

$$
\begin{aligned}
R_{E} & =-\sum_{m=0}^{1} \kappa_{m} \frac{(1-m) !}{(1+m) !} \sum_{p, p^{\prime}=0}^{1} F_{1, m, p}\left(I^{\prime}\right) \\
& \times \sum_{q, q^{\prime}=-\infty}^{+\infty} \chi_{1-2 p+q}^{1,1-2 p}(e) \chi_{1-2 p^{\prime}+q^{\prime}}^{1,1-2 p^{\prime}}\left(e^{\prime}\right) \times \cos \left[\left(1-2 p^{\prime}+q^{\prime}\right) \lambda^{\prime}-(1-2 p+q) \lambda\right. \\
& \left.-q^{\prime} \varpi^{\prime}+q \varpi-\left(1-2 p^{\prime}-m\right) \Omega^{\prime}+(1-2 p-m) \Omega\right]
\end{aligned}
$$

e

$$
\begin{aligned}
R_{I} & =-\sum_{m=0}^{1} \kappa_{m} \frac{(1-m) !}{(1+m) !} \sum_{p, p^{\prime}=0}^{1} F_{1, m, p}\left(I^{\prime}\right) \\
& \times \sum_{q, q^{\prime}=-\infty}^{+\infty} \chi_{1-2 p+q}^{-2,1-2 p}(e) \chi_{1-2 p^{\prime}+q^{\prime}}^{1,1-2 p^{\prime}}\left(e^{\prime}\right) \times \cos \left[\left(1-2 p^{\prime}+q^{\prime}\right) \lambda^{\prime}-(1-2 p+q) \lambda\right. \\
& \left.-q^{\prime} \varpi^{\prime}+q \varpi-\left(1-2 p^{\prime}-m\right) \Omega^{\prime}+(1-2 p-m) \Omega\right] .
\end{aligned}
$$


Os coeficientes $b_{i+1 / 2}^{(j)}(\alpha)$ que aparecem na expressão (2.12) são os coeficientes de Laplace, defindos por

$$
\frac{1}{2} b_{s}^{(j)}(\alpha)=\frac{1}{2 \pi} \int_{0}^{2 \pi} \frac{\cos j \varphi d \varphi}{\left(1-2 \alpha \cos \varphi+\alpha^{2}\right)^{s}}, \quad s=1 / 2,3 / 2, \ldots
$$

Os coeficientes (2.15) também podem ser escritos, como uma série hipergeométrica, da seguinte forma

$$
\begin{aligned}
\frac{1}{2} b_{s}^{(j)}(\alpha) & =\frac{s(s+1) \cdots(s+j-1)}{1 \cdot 2 \cdot 3 \cdots j} \alpha^{j}\left[1+\frac{s(s+j)}{1(j+1)} \alpha^{2}\right. \\
& \left.+\frac{s(s+1)(s+j)(s+j+1)}{1 \cdot 2(j+1)(j+2)} \alpha^{4}+\cdots\right]
\end{aligned}
$$

Devemos notar que a série (2.16), assim como suas derivadas, são sempre convergentes para $\alpha<1$. Notemos também na expansão (2.13,) não aparecem os coeficientes de Laplace. Ainda com respeito aos coeficientes de Laplace definidos em (2.15) ou (2.16), temos o seguinte:

Lema 1. Sejam $b_{s}^{(j)}(\alpha)$ os coeficientes de Laplace definidos em (2.15); então, para quaisquer que sejam $\alpha \in(0,1), s \in \mathbb{R}_{+} \backslash \mathbb{N}$ e $j \in \mathbb{N}$ tem-se

$$
b_{s}^{(j)}(\alpha)>b_{s}^{(j+1)}(\alpha)
$$

Demonstração. Seja $s \in(0,1)$, denotemos

$$
s_{j}=s(s+1) \cdots(s+j-1)=\frac{(s+j-1) !}{(s-1) !}
$$

de (2.16) obemos

$$
b_{s}^{(j)}(\alpha)-b_{s}^{(j+1)}=\frac{s_{j}}{j !} \alpha^{j}\left[\left(1-\frac{s+j}{j+1} \alpha\right)+\frac{s}{1} \frac{s+j}{j+1} \alpha^{2}\left(1-\frac{s+j+1}{j+2} \alpha+\cdots\right)>0\right.
$$

Além disso, temos as seguintes relações

$$
\begin{aligned}
b_{s+1}^{(j)} & =\frac{(s+j)\left(1+\alpha^{2}\right)}{s\left(1-\alpha^{2}\right)} b_{s}^{(j)}-2 \frac{j-s+1}{s} \frac{\alpha}{\left(1-\alpha^{2}\right)^{2}} b_{s}^{(j+1)} \\
b_{s}^{(j+2)} & =\frac{j+1}{j-s+2}\left(\alpha+\frac{1}{\alpha} b_{s}^{(j+1)}-\frac{j+s}{j-s+2} b_{s}^{(j)}\right.
\end{aligned}
$$

donde

$$
b_{s+1}^{(j)}(\alpha)-b_{s+1}^{(j+1)}=\frac{j b_{s}^{(j)}+(j+1) b_{s}^{(j+1)}}{s(1+\alpha)^{2}}+\frac{b_{s}^{(j)}-b_{s}^{(j+1)}}{(1+\alpha)^{2}}
$$


desta última igualdade segue que $(2.18)$ é verdadeira para todo $s \in \mathbb{R}_{+} \backslash \mathbb{N}$

As funções que dependem das inclinações nas expressões (2.12) e (2.13) são definidas por

$$
\begin{aligned}
F_{s-2 n, m, p}(I) & =\frac{1}{2^{s-2 n}(s-2 n) !} \times \sum_{t=0}^{\min (p,[(s-2 n-m) / 2])} \\
& \times \frac{(2 s-4 n-2 t) !}{(s-2 n-m-2 t) !}\left(\begin{array}{c}
s-2 n \\
t
\end{array}\right) \\
& \times \sin ^{s-2 n-m-2 t}(I) \sum_{g=0}^{m}\left(\begin{array}{c}
m \\
g
\end{array}\right) \frac{\cos ^{g}(I)}{2^{s-2 n-2 t}} \\
& \times \min (p-t, s-2 n-m-2 t+g) \\
& \times\left(\begin{array}{c}
c-2 n-m-2 t+g \\
p-t-g
\end{array}\right)(-1)^{[(s-2 n-m) / 2]}
\end{aligned}
$$

onde $s-2 n=1$ em $(2.13)$ e os os colchetes $[(s-2 n-m) / 2]$ indicam a parte inteira da expressão entre eles.

As funções $\chi_{c}^{a, b}$, que dependem das excentricidades, são os coeficientes de Hansen (ver,Hughes (1981)) definidos por

$$
\chi_{c}^{a, b}(e)=e^{|c-b|} \sum_{\sigma=0}^{\infty} \chi_{\sigma+\alpha, \sigma+\beta}^{a, b} e^{2 \sigma},
$$

devemos observar que na expressão acima temos $\alpha=\max (0, c-b), \beta=\max (0, b-c)$, e os termos $\chi_{c, d}^{a, b}$ são os operadores de Newcomb definidos, recursivamente, por (ver,Hughes (1981)).

$$
\begin{aligned}
\chi_{0,0}^{a, b} & =1 \\
\chi_{1,0}^{a, b} & =b-\frac{a}{2}
\end{aligned}
$$

No caso $d=0$, temos

$$
4 c \chi_{c, 0}^{a, b}=2(2 b-a) \chi_{c-1,0}^{a, b+1}+(b-a) \chi_{c-2,0}^{a, b+2} .
$$


Para $d \neq 0$, temos

$$
\begin{aligned}
4 d \chi_{c, d}^{a, b} & =-2(2 b+a) \chi_{c, d-1}^{a, b-1}-(b+a) \chi_{c, d-2}^{a, b-2} \\
& -(c-5 d+4+4 b+a) \chi_{c-1, d-1}^{a, b} \\
& +2(c-d+b) \sum_{j \geqq 2}(-1)^{j}\left(\begin{array}{c}
\frac{3}{2} \\
j
\end{array}\right) \chi_{c-j, d-j}^{a, b}
\end{aligned}
$$

Observemos que se $c<0$ ou $d<0$, então $\chi_{c, d}^{a, b}=0$. Se $d>c$ então $\chi_{c, d}^{a, b}=\chi_{d, c}^{a,-b}$.

Foi escrito um código computacional em linguagem Fortran para calcular os termos da função perturbadora até quarta ordem em excentricidades.

\subsection{Uso da Função Perturbadora}

Como foi dito antes, as expansões (2.12) e (2.13) da função perturbadora contêm um número muito grande de somas. Contudo, existem muitas relações entre os coeficientes no cosseno do argumento e as potências das excentricidades que nos permitem reduzir o número de somas nas respectivas expansões.

Observamos que em relação a expansão (2.12), temos as seguintes propriedades, vindas das propriedades (2.4):

- As mais baixas potências de $\sin (I)$ e $\sin \left(I^{\prime}\right)$ que surgem são os valores absolutos dos coeficientes de $\Omega$ e $\Omega^{\prime}$, respectivamente, no cosseno do argumento

- A soma das potência das inclinações é sempre um número par, com isso, a expansão nunca contém uma potência isolada de uma única inclinação.

De fato, as expansões da parte direta e indireta da função perturbadora, dadas por (2.12) e (2.13), respectivamente, são séries infinitas. Todavia, nas questões práticas estamos interessados em termos que nos são apropriados para um determinado problema em particular. Uma vez que selecionamos os termos de interesse, consideramos que todos os outros termos contribuem apenas com efeitos de curto período.

Uma questão que surge é a seguinte: Como proceder para determinarmos os termos apropriados?

1. Primeiramente devemos definir quais ângulos $\Phi$ são requeridos no problema a ser estudado, dado pela física do problema. 
2. Determinação da ordem do argumento. Isto é dado por $N=\left|j_{1}+j_{2}\right|$, ou seja, é o valor absoluto da soma dos coeficientes das longitudes $\lambda^{\prime}$ e $\lambda$ em $\Phi$.

3. Na expansão de $R_{D}$, devemos procurar os termos de ordem apropriada, daí determinar quais valores de $j$ correspondem ao argumento $\Phi$ "escolhido".

4. Calculamos as combinações dos coeficientes de Laplace para os valores de $j$ que dão os termos de interesse.

5. Decidir se a perturbação é externa ou interna, ou seja, quem é o perturbador.

6. Procuramos os termos apropriados na expansão de $R_{E}$ ou $R_{I}$.

Vale notar que devemos considerar outros termos também. Por exemplo, se estivéssemos interessados em estudar a ressonância 3:1, até quarta ordem, então deveríamos considerar também as contribuições dos termos $6 \lambda^{\prime}-3 \lambda$ e seu negativo. Além disso, devemos considerar também os termos seculares.

\subsection{Ressonâncias no sistema GJ581}

Em 2010, a comunidade cientifica anunciou a existência de uma Super-Terra na Zona Habitável da estrela Gliese 581 (ver, Vogt et al. (2010)). Mais recentemente, foi publicado um outro artigo em que os autores nos dão mais evidências a respeito da existência desta Super-Terra(ver, Vogt et al. (2012)).

Do ponto de vista dinâmico, a Zona Habitável da estrela Gliese 581 é muito rica. De fato, figura (2.2) mostra várias ressonâncias de movimentos médios agindo como fonte de instabilidade na zona habitável. A localização de algumas ressonâncias selecionadas coincidindo com as regiões caóticas são indicadas pela linha verticais ( ver, Tadeu dos Santos et al. (2012)). Nesta seção, destacamos algumas destas ressonâncias de dois e de três corpos. Em particular, no caso das ressonâncias de três corpos será feito um estudo detalhado no capítulo (3). Também damos aqui parte da função perturbadora para as ressonâncias; 2/1 entre os planetas GJ581d e o planeta teste, 3/1 entre o planeta teste e GJ581c e 5/1 entre os planetas GJ581 c e d.

Abaixo seguem os termos da Função Perurbadora, com os argumento e seus respectivos coeficientes. Como foi dito antes, estes coeficientes foram calculados usando um código em 

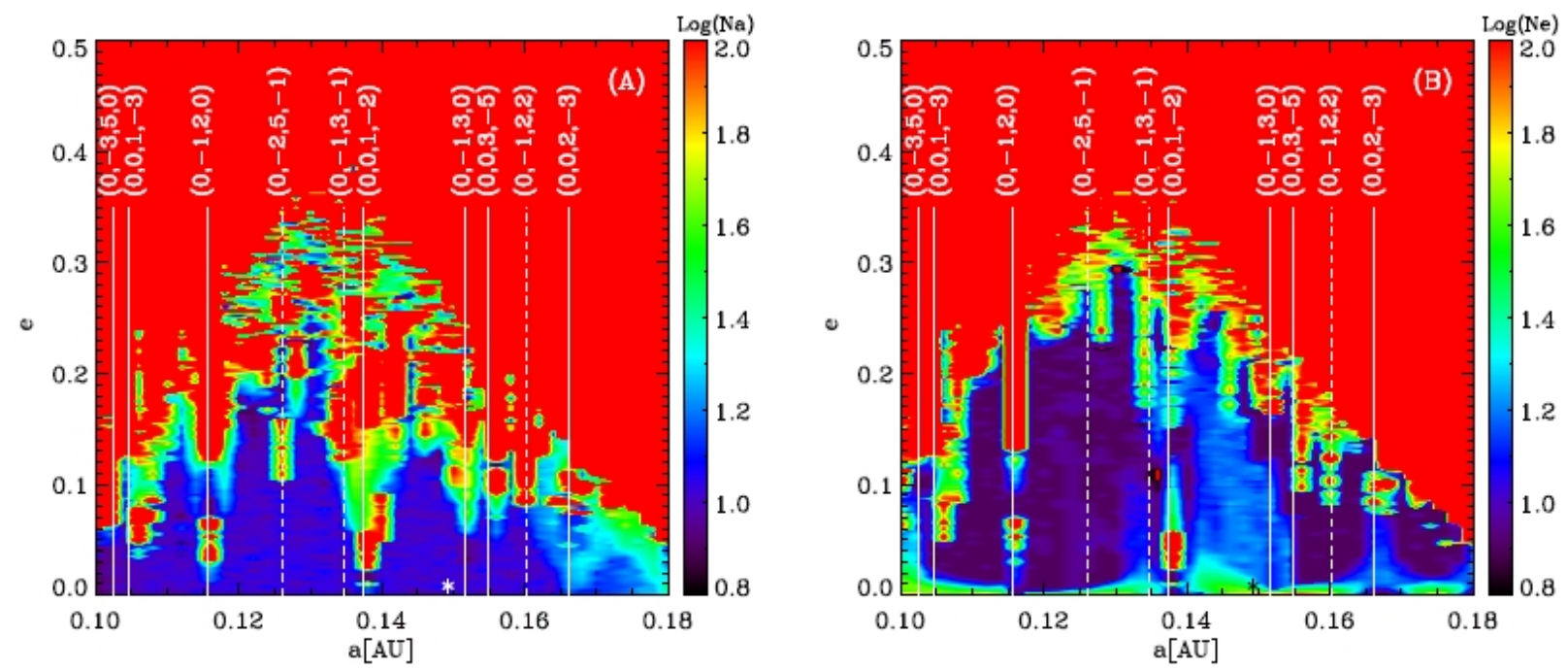

Figura 2.2: Mapa dinâmico da região onde se encontra o planeta GJ581g. Mostra-se o logaritmo do número espectral correspondentes às variações do semi-eixo maior(A) e excentricidade (B), respectivamente. As linhas verticais representam as ressonâncias mais importantes do intervalo.

\begin{tabular}{|c|c|}
\hline Ressonância 2/1 & Parte Secular \\
\hline \hline & Coeficiente \\
\hline Argumento & $1.2+0.8776\left(e_{2}{ }^{2}+e_{3}{ }^{2}\right)+14.99 e_{3}{ }^{2} e_{1}{ }^{2}+5.50 e_{3}{ }^{4}+2.43 e_{2}{ }^{4}$ \\
\hline 0 & $-1.46 e_{3} e_{2}-14.34 e_{2}{ }^{3} e_{3}-17.67 e_{3}{ }^{3} e_{2}$ \\
\hline$\varpi_{3}-\varpi_{2}$ & $6.7557 e_{2}{ }^{2} e_{3}{ }^{2}$ \\
\hline $2 \varpi_{3}-2 \varpi_{2}$ & \\
\hline
\end{tabular}

Tabela 2.1 - Parte Secular da Função perturbadora para os planetas GJ581d e o planeta teste.

linguagem Fortran, seguindo o artigo (Ellis e Murray (2000)), para ordem máxima igual a quatro. Devemos notar também que estamos considerando o caso planar, portanto os termos que envolvem as inclinações não foram considerados.

\subsubsection{Ressonância 2/1 entre o planeta GJ581d e o planeta teste}

Por conveniência de notação, vou considerar o negativo da ressonância, assim o ângulo crítico é dado por $2 \lambda_{3}-\lambda_{2}-\theta$. Nas tabelas (2.1), (2.2) e (2.3), são mostrados os argumentos e os respectivos coeficientes da expansão da Função Perturbadora para os planetas GJ581d e teste. Os coeficientes foram calculados para $\alpha=a_{2} / a_{3}=0.7334$ 


\begin{tabular}{|c|c|}
\hline Ressonância $2 / 1$ & Parte Direta \\
\hline \hline Argumento & Coeficiente \\
\hline $2 \lambda_{3}-\lambda_{2}-\varpi_{2}$ & $-1.8916250 e_{2}-0.3988658 e_{2}^{3}-2.5865382 e_{2} e_{3}{ }^{2}$ \\
\hline $2 \lambda_{3}-\lambda_{2}-\varpi_{3}$ & $2.3126602 e_{3}+6.4324978 e_{2}{ }^{2} e_{3}+0.3831889 e_{3}{ }^{3}$ \\
\hline $2 \lambda_{3}-\lambda_{2}+\varpi_{3}-2 \varpi_{2}$ & $-3.243878 e_{2}{ }^{2} e_{3}$ \\
\hline $2 \lambda_{3}-\lambda_{2}-2 \varpi_{3}+\varpi_{2}$ & $-4.6036128 e_{2} e_{3}{ }^{2}$ \\
\hline $4 \lambda_{3}-2 \lambda_{2}-2 \varpi_{2}$ & $3.81177 e_{2}{ }^{2}+9.3334 e_{2}{ }^{4}-25.16 e_{2}{ }^{2} e_{3}{ }^{2}$ \\
\hline $4 \lambda_{3}-2 \lambda_{2}-\varpi_{3}-\varpi_{2}$ & $-9.62 e_{2} e_{3}-9.5721 e_{2}{ }^{3} e_{3}+7.2524 e_{2} e_{3}{ }^{3}$ \\
\hline $4 \lambda_{3}-2 \lambda_{2}-2 \varpi_{3}$ & $6.00 e_{2} e_{3}{ }^{2}+19.41 e_{2}{ }^{2} e_{3}{ }^{2}+1.1572 e_{3}{ }^{4}$ \\
\hline $4 \lambda_{3}-2 \lambda_{2}+\varpi_{3}-3 \varpi_{2}$ & $6.06 e_{2}{ }^{3} e_{3}$ \\
\hline $4 \lambda_{3}-2 \lambda_{2}-3 \varpi_{3}+\varpi_{2}$ & $-10.89 e_{2} e_{3}{ }^{3}$ \\
\hline $6 \lambda_{3}-3 \lambda_{2}-3 \varpi_{2}$ & $-9.30 e_{3}{ }^{3}$ \\
\hline $6 \lambda_{3}-3 \lambda_{2}-\varpi_{3}-2 \varpi_{2}$ & $35.40 e_{2}{ }^{2} e_{3}$ \\
\hline $6 \lambda_{3}-3 \lambda_{2}-2 \varpi_{3}-\varpi_{2}$ & $-44.75 e_{2} e_{3}{ }^{2}$ \\
\hline $6 \lambda_{3}-3 \lambda_{2}-3 \varpi_{3}$ & $18.7570 e_{3}{ }^{3}$ \\
\hline $8 \lambda_{3}-4 \lambda_{2}-4 \varpi_{2}$ & $24.72 e_{2}{ }^{4}$ \\
\hline $8 \lambda_{3}-4 \lambda_{2}-\varpi_{3}-3 \varpi_{2}$ & $-125.6732 e_{2}{ }^{3} e_{3}$ \\
\hline $8 \lambda_{3}-4 \lambda_{2}-2 \varpi_{3}-2 \varpi_{2}$ & $239.1220 e_{2}{ }^{2} e_{3}{ }^{2}$ \\
\hline $8 \lambda_{3}-4 \lambda_{2}-3 \varpi_{3}-1 \varpi_{2}$ & $-196.8334 e_{2} e_{3}{ }^{3}$ \\
\hline $8 \lambda_{3}-4 \lambda_{2}-4 \varpi_{3}$ & $63.66 e_{3}{ }^{4}$ \\
\hline
\end{tabular}

Tabela 2.2 - Parte Direta da Função perturbadora para os planetas GJ581d e o planeta teste.

\begin{tabular}{|c|c|}
\hline Ressonância 2/1 & Parte Indireta \\
\hline \hline Argumento & Coeficiente \\
\hline $2 \lambda_{3}-\lambda_{2}-\varpi_{3}$ & $-2 e_{3}+e_{2}^{2} e_{3}+\frac{3}{2} e_{3}{ }^{3}$ \\
\hline $4 \lambda_{3}-2 \lambda_{2}-3 \varpi_{3}+\varpi_{2}$ & $-\frac{8}{3} e_{2} e_{3}{ }^{3}$ \\
\hline
\end{tabular}

Tabela 2.3 - Parte Indireta da Função perturbadora para os planetas GJ581d e o planeta teste. 


\begin{tabular}{|c|c|}
\hline Ressonância 3/1 & Parte Secular \\
\hline \hline & Coeficiente \\
\hline Argumento & $1.05+0.12\left(e_{2}{ }^{2}+e_{1}^{2}\right)+0.033 e_{1}^{4}+0.2440 e_{2}{ }^{4}+0.4325 e_{1}{ }^{2} e_{2}{ }^{2}$ \\
\hline 0 & $0.1185 e_{1} e_{2}-0.2956 e_{1}^{3} e_{2}-0.5268 e_{2}{ }^{3} e_{1}$ \\
\hline$\varpi_{2}-\varpi_{1}$ & $0.10 e_{2}{ }^{2} e_{1}{ }^{2}$ \\
\hline $2 \varpi_{2}-2 \varpi_{1}$ & \\
\hline
\end{tabular}

Tabela 2.4 - Parte Indireta da Função perturbadora para os planetas GJ581c e o planeta teste.

\begin{tabular}{|c|c|}
\hline Ressonância $3 / 1$ & Parte Direta \\
\hline \hline Argumento & Coeficiente \\
\hline $3 \lambda_{2}-\lambda_{1}-2 \varpi_{1}$ & $0.4974549 e_{1}{ }^{2}+0.4509429 e_{1}{ }^{4}-2.724558 e_{1}{ }^{2} e_{2}{ }^{2}$ \\
\hline $3 \lambda_{2}-\lambda_{1}-\varpi_{2}-\varpi_{1}$ & $-1.9397273 e_{1} e_{2}+0.4422639 e_{1}{ }^{3} e_{2}+3.3109792 e_{2}{ }^{3} e_{1}$ \\
\hline $3 \lambda_{2}-\lambda_{1}-2 \varpi_{1}$ & $1.8360082 e_{2}{ }^{2}+0.2519123 e_{1}{ }^{2} e_{2} 2-1.2364354 e_{2}{ }^{4}$ \\
\hline $3 \lambda_{2}-\lambda_{1}+\varpi_{2}-3 \varpi_{1}$ & $0.5648785 e_{1}{ }^{3} e_{2}$ \\
\hline $3 \lambda_{2}-\lambda_{1}-3 \varpi_{2}+\varpi_{1}$ & $-0.7164743 e_{1} e_{2}{ }^{3}$ \\
\hline $6 \lambda_{2}-3 \lambda_{1}-3 \varpi_{1}$ & $-0.3446 e_{1}{ }^{3}$ \\
\hline $6 \lambda_{2}-3 \lambda_{1}-\varpi_{2}-2 \varpi_{1}$ & $2.09 e_{1}{ }^{2} e_{2}$ \\
\hline $6 \lambda_{2}-3 \lambda_{1}-2 \varpi_{2}-\varpi_{1}$ & $-4.2451 e_{1} e_{2}{ }^{2}$ \\
\hline $6 \lambda_{2}-3 \lambda_{1}-3 \varpi_{2}$ & $2.8435 e_{1} e_{2}{ }^{2}$ \\
\hline
\end{tabular}

Tabela 2.5 - Parte Direta da Função perturbadora para os planetas GJ581c e o planeta teste.

\subsubsection{Ressonância 3/1 Entre o Planeta GJ581c e o Planeta Teste}

As tabelas (2.4), (2.5) e (2.6) mostram os argumentos e os respectivos coeficientes da expansão da função perturbadora para os planetas GJ581c e o planeta teste. Observamos que nesta expansão temos $\alpha=a_{1} / a_{2}=0.4555$ e o ângulo crítico é dado por $3 \lambda_{2}-\lambda_{1}-\theta$

\subsubsection{Ressonância 5/1 Entre os Planetas GJ581d e GJ581c}

As tabelas (2.7), (2.8) e (2.9) mostram a expansão da função perturbadora para os planetas GJ581d e GJ581c, cujo ângulo crítico é dadao por $5 \lambda_{3}-\lambda_{1}-\theta$., e $\alpha=a_{1} / a_{3}=$ 0.3471 . 


\begin{tabular}{|c|c|}
\hline Ressonância 3/1 & Parte Indireta \\
\hline \hline Argumento & Coeficiente \\
\hline $3 \lambda_{2}-\lambda_{1}-2 \varpi_{2}$ & $-\frac{27}{8} e_{2}^{2}+\frac{27}{16} e_{1}^{2} e_{2}^{2}+\frac{27}{8} e_{2}^{4}$ \\
\hline
\end{tabular}

Tabela 2.6 - Parte Indireta da Função perturbadora para os planetas GJ581c e o planeta teste.

\begin{tabular}{|c|c|}
\hline Ressonância 5/1 & Parte Secular \\
\hline \hline & Coeficiente \\
\hline Argumento & $1.03+0.052\left(e_{3}{ }^{2}+e_{1}{ }^{2}\right)+0.006 e_{1}{ }^{4}+0.083 e_{3}{ }^{4}+0.126 e_{1}{ }^{2} e_{3}{ }^{2}$ \\
\hline 0 & $0.282486 e_{1} e_{3}-0.06373 e_{1}^{3} e_{3}-0.1353 e_{1} e_{3}{ }^{3}$ \\
\hline$\varpi_{3}-\varpi_{1}$ & $0.01 e_{1}{ }^{2} e_{3}{ }^{2}$ \\
\hline $2 \varpi_{3}-2 \varpi_{1}$ & \\
\hline
\end{tabular}

Tabela 2.7 - Parte Secular da Função perturbadora para os planetas GJ581d e GJ581c.

\begin{tabular}{|c|c|}
\hline Ressonância $5 / 1$ & Parte Direta \\
\hline \hline Argumento & Coeficiente \\
\hline $5 \lambda_{3}-\lambda_{1}-4 \varpi_{1}$ & $0.0749 e_{1}{ }^{4}$ \\
\hline $5 \lambda_{3}-\lambda_{1}-\varpi_{3}-3 \varpi_{1}$ & $-0.7737 e_{1}{ }^{3} e_{3}$ \\
\hline $5 \lambda_{3}-\lambda_{1}-2 \varpi_{3}-2 \varpi_{1}$ & $2.9692 e_{3}{ }^{3} e_{1}$ \\
\hline $5 \lambda_{3}-\lambda_{1}-3 \varpi_{3}-\varpi_{1}$ & $-5 e_{3}{ }^{3} e_{1}$ \\
\hline $5 \lambda_{3}-\lambda_{1}-4 \varpi_{3}$ & $3.099 e_{3}{ }^{4}$ \\
\hline
\end{tabular}

Tabela 2.8 - Parte Direta da Função perturbadora para os planetas GJ581d e GJ581c.

\begin{tabular}{|c|c|}
\hline Ressonância 5/1 & Parte Indireta \\
\hline \hline Argumento & Coeficiente \\
\hline \hline $5 \lambda_{3}-\lambda_{1}-4 \varpi_{3}$ & $\frac{-3125}{384} e_{3}^{4}$ \\
\hline
\end{tabular}

Tabela 2.9 - Parte Indireta da Função perturbadora para os planetas GJ581d e GJ581c. 


\subsection{Variáveis de Delaunay e Poincaré}

\subsubsection{Variáveis de Delaunay}

Visto que o problema planetário pode ser expresso como a perturbação do Hamiltoniano integrável $H_{0}$, e além disso, se estamos interessados em estudar a variação temporal dos elementos órbitais, frequentemente usamos como variáveis açoes $L, G$ e $\Theta$, e ângulos $l, g$ e $\theta$, conhecidas como variáveis canônicas de Delaunay.

$$
\begin{array}{rlrl}
L_{i} & =\beta_{i} \sqrt{\mu_{i} a_{i}}, & l_{i}=M_{i} \\
G_{i}=\beta_{i} \sqrt{\mu_{i} a_{i}\left(1-e_{i}^{2}\right),} & g_{i}=\omega_{i} \\
\Theta_{i}=G \cos \left(I_{i}\right), & \theta_{i}=\Omega_{i}
\end{array}
$$

Onde $a_{i}, e_{i}, I_{i}, M_{i}, \omega_{i}$ e $\Omega_{i}$ são semieixo, excentricidade, inclinação, anomalia média, argumento do pericentro e longitude do nodo do i-ésimo planeta, respectivamente,

$$
\mu_{i}=G\left(m_{0}+m_{i}\right) \quad \text { e } \quad \beta_{i}=\frac{m_{0} m_{i}}{m_{0}+m_{i}}
$$

onde $m_{0}$ é a massa do corpo central.

Contudo, as variáveis de Delaunay não são muito convenientes para sistemas com baixas excentricidades e inclinações. De fato, se considerarmos um plano órbital com $I=0$, este plano coincidirá com o plano de referência, de modo que não mais estará definida a linha dos nodos. Logo a longitude dos nodos também não estará definida. Além disso, se a órbita tem $e=0$, a direção do pericentro não está definida. Para superarmos o problema de pequenas excentricidades e inclinações, utilizam-se as variáveis canônicas de Poincaré

\subsubsection{Variáveis de Poincaré}

Para obter tal transformação Poincaré primeiro realizou a transformação linear nas variáveis nos ângulos de Delaunay e depois estendeu às ações. Obtendo assim, a seguinte transformação canônica

$$
\begin{array}{rlrl}
\Lambda_{i} & =L_{i} ; & \lambda=l_{i}+g_{i}+\theta_{i} \\
P_{i}=L_{i}-G_{i}=L_{i}\left(1-\sqrt{1-e^{2}}\right) ; & h_{i}=-g_{i}-\theta_{i} \equiv-\varpi_{i} \\
Z_{i}=G_{i}-\Theta=L_{i} \sqrt{\left(1-e_{i}^{2}\right)}\left(1-\cos I_{i}\right) ; & \zeta_{i}=-\theta_{i} \equiv-\Omega_{i}
\end{array}
$$


Todavia, esta transformação ainda tem um problema: ela não está definida para excentricidades ou inclinações zero. Assim, Poincaré fez uma nova mudança de coordenadas. Por conveniência iremos omitir os índices nas espressões abaixo.

$$
\begin{array}{rlrl}
\Lambda & =L ; & \lambda & l+g+\theta \\
\xi=\sqrt{2 \Lambda} \sqrt{1-\sqrt{1-e^{2}}} \cos \varpi ; & \eta=-\sqrt{2 \Lambda} \sqrt{1-\sqrt{1-e^{2}}} \sin \varpi \\
p=\sqrt{2 \Lambda} \sqrt{\left.\sqrt{1-e^{2}}(1-\cos I)\right)} \cos \Omega ; & q=-\sqrt{2 \Lambda} \sqrt{\sqrt{1-e^{2}}(1-\cos I)} \sin \Omega
\end{array}
$$

Agora usando variáveis complexas, podemos fazer outra transformação em (2.28) obtendo $(x,-i \bar{x})$ e $(y,-i \bar{y})$, onde

$$
\begin{aligned}
& x=\frac{1}{\sqrt{2}}(\xi-i \eta)=\sqrt{\Lambda} \sqrt{1-\sqrt{1-e^{2}}} \exp i \varpi ; \quad-i \bar{x} \\
& y=\frac{1}{\sqrt{2}}(p-i q)=\sqrt{\Lambda} \sqrt{\sqrt{1-e^{2}}(1-\cos I)} \exp i \Omega ; \quad-i \bar{y}
\end{aligned}
$$

Onde a barra denota o complexo conjugado. Devemos observar que no caso $e=0$ a $I=0$, tem-se $x=0$ e $y=0$, de modo que, mesmo neste caso, as variáveis de Poincaré são bem definidas.

\subsection{Conexão entre as variáveis de Poincaré e os elementos elípticos} clássicos

Note que as variávei de Poincaré (2.28) e (2.29) estão associadas aos elementos elíptcos clássicos dados por

$$
\begin{aligned}
& z=\tilde{k}+i \tilde{h}=e \exp i \varpi \\
& \zeta=\tilde{q}+i \tilde{p}=\sin \left(\frac{I}{2}\right) \exp i \Omega
\end{aligned}
$$

Através da relação 


$$
\begin{aligned}
& z=x \sqrt{\frac{2}{\Lambda}}\left(1-\frac{x \bar{x}}{2 \Lambda}\right)^{1 / 2} \\
& \zeta=y \frac{1}{\sqrt{2 \Lambda}}\left(1-\frac{x \bar{x}}{\Lambda}\right)^{-1 / 2}
\end{aligned}
$$

Note que, para pequenas excentricidades, de (2.29) tem-se que

$$
\begin{aligned}
& x \approx \sqrt{\Lambda} \sqrt{1-\left(1-\frac{e^{2}}{2}\right)} \exp i \varpi \approx \sqrt{\frac{\Lambda}{2}} e \exp i \varpi=\sqrt{\frac{\Lambda}{2}} z \\
& y \approx \sqrt{2 \Lambda} \sqrt{1-\frac{1}{4} e^{2}} \sin (I / 2) \exp (i \Omega) \approx \sqrt{2 \Lambda} \sin (I / 2) \exp (i \Omega)=\sqrt{2 \Lambda} \zeta
\end{aligned}
$$

\subsection{Forma Hamiltoniana Para o Sistema Perturbado}

Sabemos que o Hamiltoniano, para o problema de 4 corpos em coordenadas astrocêntricas é dado por

$$
H=\sum_{i=1}^{3}\left(\frac{\left\|\mathbf{p}_{i}\right\|^{2}}{\beta_{i}}-\frac{\mu_{i} \beta_{i}}{\left\|\mathbf{r}_{i}\right\|}\right)-G \sum_{i=1, i<j}^{3} \frac{m_{i} m_{j}}{\Delta_{i j}}+\sum_{i=1, i<j}^{3} \frac{\mathbf{p}_{i} \times \mathbf{p}_{j}}{m_{0}}
$$

onde os $\mathbf{r}_{i}$ são os vetores posição relativos, $\mathbf{p}_{i}=m_{i}(d \boldsymbol{\rho} / d t)$ são os vetores conjugados à $\mathbf{r}_{i}$, e $\boldsymbol{\rho}_{i}$ são os vetores relativos ao centro de massa do sistema.

Escrevendo o Hamiltoniano (2.34) nas variáveis (2.27) temos

$$
H=H_{0}-R\left(P_{1}, P_{2}, P_{3}, \Lambda_{1}, \Lambda_{2}, \Lambda_{3}, \lambda_{1}, \lambda_{2}, \lambda_{3}, \varpi_{1}, \varpi_{2}, \varpi_{3}\right)
$$

onde $R$ é a função perturbadora e $H_{0}$ é dado por

$$
H_{0}=-\frac{\beta_{1}^{3} \mu_{1}^{2}}{2 \Lambda_{1}^{2}}-\frac{\beta_{2}{ }^{3} \mu_{2}^{2}}{2 \Lambda_{2}^{2}}-\frac{\beta_{3}^{3} \mu_{3}^{2}}{2 \Lambda_{3}^{2}}
$$

Agora denotando $R$ por $H_{1}$ e tomando $\epsilon=m_{i} / m_{0}$ podemos escrever o Hamiltoniano (2.35) da seguinte forma

$$
H=H_{0}+\epsilon H_{1}
$$

$\mathrm{e}$

$$
H_{1}=H_{1}^{1}+H_{1}^{2}+H_{1}^{3}+H_{1}^{4}+H_{1}^{5}+H_{1}^{6}+H_{1}^{7}
$$


Os $n_{1}, n_{2}$ e $n_{3}$ são os movimentos médio dos planetas GJ581c, o planeta teste e o planeta GJ581d, respectivamente. Os $\beta_{i}^{\prime} s$ e $\mu_{i}^{\prime} s$ são como em (2.26) e os $H_{1}^{i}$, para $i=1, \cdots, 7$; são dados por $(2.39),(2.40),(2.41),(2.42),(2.43),(2.44)$ e (2.45), respectivamente.

$$
\begin{aligned}
& H_{1}^{1}=\frac{0.1 \beta_{2}^{2} G^{2} M_{2} P_{1} P_{2}\left(P_{1}-2 . \Lambda_{1}\right)\left(P_{2}-2 . \Lambda_{2}\right) \cos \left(2 \varpi_{1}-2 \varpi_{2}\right)}{\Lambda_{1}^{2} \Lambda_{2}^{4}} \\
& +\frac{4.2451 \beta_{2}^{2} G^{2} M_{2} P_{2} \sqrt{P_{1}\left(2-\frac{P_{1}}{\Lambda_{1}}\right)}\left(P_{2}-2 . \Lambda_{2}\right) \cos \left(3 \lambda_{1}-6 \lambda_{2}+\varpi_{1}+2 \varpi_{2}\right)}{\sqrt{\Lambda_{1} \Lambda_{2}^{4}}} \\
& -\frac{2.8435 \beta_{2}^{2} G^{2} M_{2} P_{2} \sqrt{P_{1}\left(2-\frac{P_{1}}{\Lambda_{1}}\right)}\left(P_{2}-2 . \Lambda_{2}\right) \cos \left(3 \lambda_{1}-6 \lambda_{2}+3 \varpi_{2}\right)}{\sqrt{\Lambda_{1}} \Lambda_{2}^{4}} \\
& -\frac{0.716474 \beta_{2}^{2} G^{2} M_{2} \sqrt{P_{1}\left(2-\frac{P_{1}}{\Lambda_{1}}\right)}\left(P_{2}\left(2-\frac{P_{2}}{\Lambda_{2}}\right)\right)^{3 / 2} \cos \left(\lambda_{1}-3 \lambda_{2}-\varpi_{1}+3 \varpi_{2}\right)}{\sqrt{\Lambda_{1}} \Lambda_{2}^{7 / 2}} \\
& -\frac{2.09 \beta_{2}^{2} G^{2} M_{2} P_{1}\left(P_{1}-2 . \Lambda_{1}\right) \sqrt{P_{2}\left(2-\frac{P_{2}}{\Lambda_{2}}\right)} \cos \left(3 \lambda_{1}-6 \lambda_{2}+2 \varpi_{1}+\varpi_{2}\right)}{\Lambda_{1}^{2} \Lambda_{2}^{5 / 2}} \\
& +\frac{0.564878 \beta_{2}^{2} G^{2} M_{2}\left(P_{1}\left(2-\frac{P_{1}}{\Lambda_{1}}\right)\right) \sqrt[3 / 2]{P_{2}\left(2-\frac{P_{2}}{\Lambda_{2}}\right)} \cos \left(\lambda_{1}-3 \lambda_{2}+3 \varpi_{1}-\varpi_{2}\right)}{\Lambda_{1}^{3 / 2} \Lambda_{2}^{5 / 2}} \\
& -\frac{0.3446 \beta_{2}^{2} G^{2} M_{2}\left(P_{1}\left(2-\frac{P_{1}}{\Lambda_{1}}\right)\right)^{3 / 2} \cos \left(3 \lambda_{1}-6 \lambda_{2}+3 \varpi_{1}\right)}{\Lambda_{1}^{3 / 2} \Lambda_{2}^{2}} \\
& H_{1}^{2}=-\frac{2.7181 \beta_{3}^{2} G^{2} M_{3} P_{3}^{2}\left(P_{3}-2 . \Lambda_{3}\right)^{2} \cos \left(\lambda_{1}-5 \lambda_{3}-4 \varpi_{3}\right)}{\Lambda_{3}^{6}} \\
& +\frac{63.66 \beta_{3}^{2} G^{2} M_{3} P_{3}^{2}\left(P_{3}-2 . \Lambda_{3}\right)^{2} \cos \left(4 \lambda_{2}-8 \lambda_{3}+4 \varpi_{3}\right)}{\Lambda_{3}^{6}} \\
& +\frac{3.099 \beta_{3}^{2} G^{2} M_{3} P_{3}^{2}\left(P_{3}-2 . \Lambda_{3}\right)^{2} \cos \left(\lambda_{1}-5 \lambda_{3}+4 \varpi_{3}\right)}{\Lambda_{3}^{6}} \\
& +\frac{0.01 \beta_{3}^{2} G^{2} M_{3} P_{1} P_{3}\left(P_{1}-2 . \Lambda_{1}\right)\left(P_{3}-2 . \Lambda_{3}\right) \cos \left(2 \varpi_{1}-2 \varpi_{3}\right)}{\Lambda_{1}^{2} \Lambda_{3}^{4}} \\
& +\frac{6.7557 \beta_{3}^{2} G^{2} M_{3} P_{2} P_{3}\left(P_{2}-2 . \Lambda_{2}\right)\left(P_{3}-2 . \Lambda_{3}\right) \cos \left(2 \varpi_{2}-2 \varpi_{3}\right)}{\Lambda_{2}^{2} \Lambda_{3}^{4}} \\
& +\frac{239.122 \beta_{3}^{2} G^{2} M_{3} P_{2} P_{3}\left(P_{2}-2 . \Lambda_{2}\right)\left(P_{3}-2 . \Lambda_{3}\right) \cos \left(4 \lambda_{2}-8 \lambda_{3}+2 \varpi_{2}+2 \varpi_{3}\right)}{\Lambda_{2}^{2} \Lambda_{3}^{4}} \\
& +\frac{4.60361 \beta_{3}^{2} G^{2} M_{3} P_{3} \sqrt{P_{2}\left(2-\frac{P_{2}}{\Lambda_{2}}\right)}\left(P_{3}-2 . \Lambda_{3}\right) \cos \left(\lambda_{2}-2 \lambda_{3}-\varpi_{2}+2 \varpi_{3}\right)}{\sqrt{\Lambda_{2}} \Lambda_{3}^{4}}
\end{aligned}
$$




$$
\begin{aligned}
H_{1}^{3} & =\frac{44.75 \beta_{3}^{2} G^{2} M_{3} P_{3} \sqrt{P_{2}\left(2-\frac{P_{2}}{\Lambda_{2}}\right)}\left(P_{3}-2 . \Lambda_{3}\right) \cos \left(3 \lambda_{2}-6 \lambda_{3}+\varpi_{2}+2 \varpi_{3}\right)}{\sqrt{\Lambda_{2}} \Lambda_{3}^{4}} \\
& -\frac{9.3 \beta_{3}^{2} G^{2} M_{3}\left(P_{3}\left(2-\frac{P_{3}}{\Lambda_{3}}\right)\right)^{3 / 2} \cos \left(3 \lambda_{2}-6 \lambda_{3}+3 \varpi_{2}\right)}{\Lambda_{3}^{7 / 2}} \\
+ & \frac{2.9692 \beta_{3}^{2} G^{2} M_{3} \sqrt{P_{1}\left(2-\frac{P_{1}}{\Lambda_{1}}\right)}\left(P_{3}\left(2-\frac{P_{3}}{\Lambda_{3}}\right)\right)^{3 / 2} \cos \left(\lambda_{1}-5 \lambda_{3}+2 \varpi_{1}+2 \varpi_{3}\right)}{\sqrt{\Lambda_{1}} \Lambda_{3}^{7 / 2}} \\
- & \frac{5 \beta_{3}^{2} G^{2} M_{3} \sqrt{P_{1}\left(2-\frac{P_{1}}{\Lambda_{1}}\right)\left(P _ { 3 } \left(2 G^{2} M_{3}\left(P_{3}\left(2-\frac{P_{3}}{\Lambda_{3}}\right)\right)^{3 / 2} \cos \left(\lambda_{1}-5 \lambda_{3}+\varpi_{1}+3 \varpi_{3}\right)\right.\right.}}{\sqrt{\Lambda_{1}} \Lambda_{3}^{7 / 2}}(2.41) \\
- & \frac{12.9265 \beta_{3}^{2} G^{2} M_{3} \sqrt{P_{2}\left(2-\frac{P_{2}}{\Lambda_{2}}\right)}\left(P_{3}\left(2-\frac{P_{3}}{\Lambda_{3}}\right)\right)^{3 / 2} \cos \left(2 \lambda_{2}-4 \lambda_{3}-\varpi_{2}+3 \varpi_{3}\right)}{\sqrt{\Lambda_{2}} \Lambda_{3}^{7 / 2}}\left(P_{3}\left(2-\frac{P_{3}}{\Lambda_{3}}\right)\right)^{3 / 2} \cos \left(4 \lambda_{2}-8 \lambda_{3}+\varpi_{2}+3 \varpi_{3}\right) \\
- & \frac{196.833 \beta_{3}^{2} G^{2} M_{3} \sqrt{P_{2}\left(2-\frac{P_{2}}{\Lambda_{2}}\right)}\left(\sqrt{\Lambda_{2}} \Lambda_{3}^{7 / 2}\right.}{\sqrt{3 / 2}}
\end{aligned}
$$




$$
\begin{aligned}
H_{1}^{4} & =-\frac{0.7737 \beta_{3}^{2} G^{2} M_{3}\left(P_{1}\left(2-\frac{P_{1}}{\Lambda_{1}}\right)\right) \sqrt[3 / 2]{P_{3}\left(2-\frac{P_{3}}{\Lambda_{3}}\right)} \cos \left(\lambda_{1}-5 \lambda_{3}+3 \varpi_{1}+\varpi_{3}\right)}{\Lambda_{1}^{3 / 2} \Lambda_{3}^{5 / 2}} \\
& +\frac{3.24388 \beta_{3}^{2} G^{2} M_{3} P_{2}\left(P_{2}-2 . \Lambda_{2}\right) \sqrt{P_{3}\left(2-\frac{P_{3}}{\Lambda_{3}}\right)} \cos \left(\lambda_{2}-2 \lambda_{3}+2 \varpi_{2}-\varpi_{3}\right)}{\Lambda_{2}^{2} \Lambda_{3}^{5 / 2}} \\
& -\frac{35.4 \beta_{3}^{2} G^{2} M_{3} P_{2}\left(P_{2}-2 . \Lambda_{2}\right) \sqrt{P_{3}\left(2-\frac{P_{3}}{\Lambda_{3}}\right)} \cos \left(3 \lambda_{2}-6 \lambda_{3}+2 \varpi_{2}+\varpi_{3}\right)}{\Lambda_{2}^{2} \Lambda_{3}^{5 / 2}} \\
+ & \frac{6.06 \beta_{3}^{2} G^{2} M_{3}\left(P_{2}\left(2-\frac{P_{2}}{\Lambda_{2}}\right)\right)^{3 / 2} \sqrt{P_{3}\left(2-\frac{P_{3}}{\Lambda_{3}}\right)} \cos \left(2 \lambda_{2}-4 \lambda_{3}+3 \varpi_{2}-\varpi_{3}\right)}{\Lambda_{2}^{3 / 2} \Lambda_{3}^{5 / 2}} \\
+ & \frac{125.673 \beta_{3}^{2} G^{2} M_{3}\left(P_{2}\left(2-\frac{P_{2}}{\Lambda_{2}}\right)\right) 3 / 2 \sqrt{P_{3}\left(2-\frac{P_{3}}{\Lambda_{3}}\right)} \cos \left(4 \lambda_{2}-8 \lambda_{3}+3 \varpi_{2}+\varpi_{3}\right)}{\Lambda_{2}^{3 / 2} \Lambda_{3}^{5 / 2}} \\
+ & \frac{0.0749 \beta_{3}^{2} G^{2} M_{3} P_{1}^{2}\left(P_{1}-2 . \Lambda_{1}\right)^{2} \cos \left(\lambda_{1}-5 \lambda_{3}+4 \varpi_{1}\right)}{\Lambda_{1}^{4} \Lambda_{3}^{2}} \\
+ & \frac{24.72 \beta_{3}^{2} G^{2} M_{3} P_{2}^{2}\left(P_{2}-2 . \Lambda_{2}\right)^{2} \cos \left(4 \lambda_{2}-8 \lambda_{3}+4 \varpi_{2}\right)}{\Lambda_{2}^{4} \Lambda_{3}^{2}}
\end{aligned}
$$




$$
\begin{aligned}
& H_{1}^{5}=\frac{\beta_{3}^{2} G^{2} M_{3} \sqrt{P_{1}\left(2-\frac{P_{1}}{\Lambda_{1}}\right)} \sqrt{P_{3}\left(2-\frac{P_{3}}{\Lambda_{3}}\right)}}{\Lambda_{1}^{5 / 2} \Lambda_{3}^{9 / 2}} \\
& \times\left(0.1353 \Lambda_{1}^{2} P_{3}^{2}-0.27 \Lambda_{3} \Lambda_{1}^{2} P_{3}+\Lambda_{3}^{2}\left(0.2825 \Lambda_{1}^{2}-0.12746 \Lambda_{1} P_{1}+0.0637 P_{1}^{2}\right)\right) \cos \left(\varpi_{1}-\varpi_{3}\right) \\
& -\frac{1 . \beta_{3}^{2} G^{2} M_{3} P_{3}\left(P_{3}-2 . \Lambda_{3}\right)}{\Lambda_{2}^{2} \Lambda_{3}^{6}} \cos \left(2 \lambda_{2}-4 \lambda_{3}+2 \varpi_{3}\right) \\
& \times\left(-1.1572 \Lambda_{2}^{2} P_{3}^{2}+2.3144 \Lambda_{3} \Lambda_{2}^{2} P_{3}+\Lambda_{3}^{2}\left(38.82 \Lambda_{2} P_{2}+6 . \Lambda_{2}^{3 / 2} \sqrt{P_{2}\left(2-\frac{P_{2}}{\Lambda_{2}}\right)}-19.41 P_{2}^{2}\right)\right) \\
& +\frac{\beta_{3}^{2} G^{2} M_{3} \sqrt{P_{2}\left(2-\frac{P_{2}}{\Lambda_{2}}\right)} \sqrt{P_{3}\left(2-\frac{P_{3}}{\Lambda_{3}}\right)}}{\Lambda_{2}^{5 / 2} \Lambda_{3}^{9 / 2}} \cos \left(2 \lambda_{2}-4 \lambda_{3}+\varpi_{2}+\varpi_{3}\right) \\
& \times\left(-7.2524 \Lambda_{2}^{2} P_{3}^{2}+14.5048 \Lambda_{3} \Lambda_{2}^{2} P_{3}+\Lambda_{3}^{2}\left(-9.62 \Lambda_{2}^{2}-19.1442 \Lambda_{2} P_{2}+9.5721 P_{2}^{2}\right)\right) \\
& +\frac{\beta_{3}^{2} G^{2} M_{3} \sqrt{P_{2}\left(2-\frac{P_{2}}{\Lambda_{2}}\right)}}{\Lambda_{2}^{5 / 2} \Lambda_{3}^{4}} \cos \left(\lambda_{2}-2 \lambda_{3}+\varpi_{2}\right) \\
& \times\left(2.58654 \Lambda_{2}^{2} P_{3}^{2}-5.17308 \Lambda_{3} \Lambda_{2}^{2} P_{3}+\Lambda_{3}^{2}\left(-1.89163 \Lambda_{2}^{2}-0.797732 \Lambda_{2} P_{2}+0.398866 P_{2}^{2}\right)\right) \\
& +\frac{\beta_{3}^{2} G^{2} M_{3} \sqrt{P_{2}\left(2-\frac{P_{2}}{\Lambda_{2}}\right)} \sqrt{P_{3}\left(2-\frac{P_{3}}{\Lambda_{3}}\right)}}{\Lambda_{2}^{5 / 2} \Lambda_{3}^{9 / 2}} \\
& \times\left(17.67 \Lambda_{2}^{2} P_{3}^{2}-35.34 \Lambda_{3} \Lambda_{2}^{2} P_{3}+\Lambda_{3}^{2}\left(-1.46 \Lambda_{2}^{2}-28.7 \Lambda_{2} P_{2}+14.4 P_{2}^{2}\right)\right) \cos \left(\varpi_{2}-\varpi_{3}\right) \Lambda_{2}^{5 / 2} \Lambda_{3}^{9 / 2} \\
& +\frac{\beta_{3}^{2} G^{2} M_{3} \sqrt{P_{3}\left(2-\frac{P_{3}}{\Lambda_{3}}\right)}}{\Lambda_{2}^{2} \Lambda_{3}^{9 / 2}} \cos \left(\lambda_{2}-2 \lambda_{3}+\varpi_{3}\right) \\
& \times\left(-1.52874 \Lambda_{2}^{2} P_{3}^{2}+3.05748 \Lambda_{3} \Lambda_{2}^{2} P_{3}+\Lambda_{3}^{2}\left(0.78526 \Lambda_{2}^{2}+14.3924 \Lambda_{2} P_{2}-7.1962 P_{2}^{2}\right)\right) \Lambda_{2}^{2} \Lambda_{3}^{9 / 2} \\
& +\frac{\beta_{3}^{2} G^{2} M_{3} P_{2}}{\Lambda_{2}^{4} \Lambda_{3}^{4}} \cos \left(2 \lambda_{2}-4 \lambda_{3}+2 \varpi_{2}\right) \\
& \times\left(9.3334 \Lambda_{3}^{2} P_{2}^{3}-37.3336 \Lambda_{2} \Lambda_{3}^{2} P_{2}^{2}+\Lambda_{2}^{2} P_{2}\left(33.5218 \Lambda_{3}^{2}+50.32 \Lambda_{3} P_{3}-25.16 P_{3}^{2}\right)\right. \\
& \left.+\Lambda_{2}^{3}\left(7.62354 \Lambda_{3}^{2}-100.64 \Lambda_{3} P_{3}+50.32 P_{3}^{2}\right)\right)
\end{aligned}
$$




$$
\begin{aligned}
& H_{1}^{6} \quad \frac{1.53698 \beta_{2}^{2} G^{2} M_{2} P_{2}^{4}}{\Lambda_{2}^{6}}-\frac{6.1479 \beta_{2}^{2} G^{2} M_{2} P_{2}^{3}}{\Lambda_{2}^{5}}+\frac{7.56488 \beta_{2}^{2} G^{2} M_{2} P_{2}^{2}}{\Lambda_{2}^{4}} \\
& -\frac{2.83395 \beta_{2}^{2} G^{2} M_{2} P_{2}}{\Lambda_{2}^{3}}-\frac{2.40198 \beta_{2}^{2} G^{2} M_{2} P_{1} P_{2}^{2}}{\Lambda_{1} \Lambda_{2}^{4}}+\frac{1.20099 \beta_{2}^{2} G^{2} M_{2} P_{1}^{2} P_{2}^{2}}{\Lambda_{1}^{2} \Lambda_{2}^{4}} \\
& +\frac{1.05 \beta_{2}^{2} G^{2} M_{2}}{\Lambda_{2}^{2}}+\frac{4.80395 \beta_{2}^{2} G^{2} M_{2} P_{1} P_{2}}{\Lambda_{1} \Lambda_{2}^{3}}-\frac{2.40198 \beta_{2}^{2} G^{2} M_{2} P_{1}^{2} P_{2}}{\Lambda_{1}^{2} \Lambda_{2}^{3}} \\
& +\frac{0.033 \beta_{2}^{2} G^{2} M_{2} P_{1}^{4}}{\Lambda_{1}^{4} \Lambda_{2}^{2}}-\frac{0.132 \beta_{2}^{2} G^{2} M_{2} P_{1}^{3}}{\Lambda_{1}^{3} \Lambda_{2}^{2}}+\frac{0.012 \beta_{2}^{2} G^{2} M_{2} P_{1}^{2}}{\Lambda_{1}^{2} \Lambda_{2}^{2}} \\
& +\frac{5.5834 \beta_{3}^{2} G^{2} M_{3} P_{3}^{4}}{\Lambda_{3}^{6}}-\frac{22.3336 \beta_{3}^{2} G^{2} M_{3} P_{3}^{3}}{\Lambda_{3}^{5}}+\frac{0.24 \beta_{2}^{2} G^{2} M_{2} P_{1}}{\Lambda_{1} \Lambda_{2}^{2}} \\
& +\frac{21.404 \beta_{3}^{2} G^{2} M_{3} P_{3}^{2}}{\Lambda_{3}^{4}}-\frac{30.2324 \beta_{3}^{2} G^{2} M_{3} P_{1} P_{3}^{2}}{\Lambda_{1} \Lambda_{3}^{4}}+\frac{15.1162 \beta_{3}^{2} G^{2} M_{3} P_{1}^{2} P_{3}^{2}}{\Lambda_{1}^{2} \Lambda_{3}^{4}} \\
& +\frac{1.8592 \beta_{3}^{2} G^{2} M_{3} P_{3}}{\Lambda_{3}^{3}}+\frac{60.4648 \beta_{3}^{2} G^{2} M_{3} P_{1} P_{3}}{\Lambda_{1} \Lambda_{3}^{3}}-\frac{30.2324 \beta_{3}^{2} G^{2} M_{3} P_{1}^{2} P_{3}}{\Lambda_{1}^{2} \Lambda_{3}^{3}} \\
& +\frac{2.22978 \beta_{3}^{2} G^{2} M_{3}}{\Lambda_{3}^{2}}+\frac{0.0057 \beta_{3}^{2} G^{2} M_{3} P_{1}^{4}}{\Lambda_{1}^{4} \Lambda_{3}^{2}}-\frac{0.0228 \beta_{3}^{2} G^{2} M_{3} P_{1}^{3}}{\Lambda_{1}^{3} \Lambda_{3}^{2}} \\
& +\frac{2.43 \beta_{3}^{2} G^{2} M_{3} P_{2}^{4}}{\Lambda_{2}^{4} \Lambda_{3}^{2}}+\frac{0.104 \beta_{3}^{2} G^{2} M_{3} P_{1}}{\Lambda_{1} \Lambda_{3}^{2}}-\frac{0.0292 \beta_{3}^{2} G^{2} M_{3} P_{1}^{2}}{\Lambda_{1}^{2} \Lambda_{3}^{2}} \\
& -\frac{9.72 \beta_{3}^{2} G^{2} M_{3} P_{2}^{3}}{\Lambda_{2}^{3} \Lambda_{3}^{2}}+\frac{8.8424 \beta_{3}^{2} G^{2} M_{3} P_{2}^{2}}{\Lambda_{2}^{2} \Lambda_{3}^{2}}+\frac{1.7552 \beta_{3}^{2} G^{2} M_{3} P_{2}}{\Lambda_{2} \Lambda_{3}^{2}} \\
& H_{1}^{7}=\frac{\beta_{2}^{2} G^{2} M_{2} \sqrt{P_{1}\left(2-\frac{P_{1}}{\Lambda_{1}}\right)} \sqrt{P_{2}\left(2-\frac{P_{2}}{\Lambda_{2}}\right)} \cos \left(\lambda_{1}-3 \lambda_{2}+\varpi_{1}+\varpi_{2}\right)}{\Lambda_{1}^{5 / 2} \Lambda_{2}^{9 / 2}} \\
& \times\left(-3.31098 \Lambda_{1}^{2} P_{2}^{2}+6.62196 \Lambda_{2} \Lambda_{1}^{2} P_{2}+\Lambda_{2}^{2}\left(-1.93973 \Lambda_{1}^{2}+0.884528 \Lambda_{1} P_{1}-0.442264 P_{1}^{2}\right)\right) \\
& +\frac{0.244 \beta_{2}^{2} G^{2} M_{2} P_{2}^{2} \sqrt{P_{1}\left(2-\frac{P_{1}}{\Lambda_{1}}\right)} \sqrt{P_{2}\left(2-\frac{P_{2}}{\Lambda_{2}}\right)}\left(P_{2}-2 . \Lambda_{2}\right)^{2} \cos \left(\varpi_{1}-\varpi_{2}\right)}{\Lambda_{1}^{5 / 2} \Lambda_{2}^{17 / 2}} \\
& \times\left(0.5268 \Lambda_{1}^{2} P_{2}^{2}-1.0536 \Lambda_{2} \Lambda_{1}^{2} P_{2}+\Lambda_{2}^{2}\left(0.1185 \Lambda_{1}^{2}-0.5912 \Lambda_{1} P_{1}+0.2956 P_{1}^{2}\right)\right) \\
& +\frac{\beta_{2}^{2} G^{2} M_{2} \cos \left(\lambda_{1}-3 \lambda_{2}+2 \varpi_{1}\right)}{\Lambda_{1}^{4} \Lambda_{2}^{6}} \\
& \times\left(-1.23644 \Lambda_{1}^{4} P_{2}^{4}+4.94574 \Lambda_{2} \Lambda_{1}^{4} P_{2}^{3}+\Lambda_{2}^{2} \Lambda_{1}^{2} P_{2}^{2}\left(-6.78175 \Lambda_{1}^{2}+4.94532 \Lambda_{1} P_{1}-2.47266 P_{1}^{2}\right)\right. \\
& \Lambda_{2}^{4} P_{1}\left(0.99491 \Lambda_{1}^{3}-1.80377 \Lambda_{1} P_{1}^{2}+1.30632 \Lambda_{1}^{2} P_{1}+0.450943 P_{1}^{3}\right) \\
& \left.+\Lambda_{1}^{2} \Lambda_{2}^{3} P_{2}\left(3.67202 \Lambda_{1}^{2}-9.89063 \Lambda_{1} P_{1}+4.94532 P_{1}^{2}\right)\right)
\end{aligned}
$$


Capítulo 3

\section{Hamiltonianos Médios Com Ressonâncias de Três}

\section{Corpos}

Este capítulo tem por finalidade mostrar os Hamiltonianos médios na vizinhança das ressonâncias de três corpos. Usou-se a teoria de Lie para perturbações como ferramenta para obter-se tais Hamiltonianos. Vale notar que de maneira geral, a teoria de Lie para perturbações é uma reformulação das teorias clássicas de Poincaré e Von Zeipel.

\subsection{Operador de Lie}

Suponhamos que temos um sistema dinâmico com $n$ graus de liberdade cujas coordenadas e momentos conjugados são $q=\left(q_{1}, q_{2}, \ldots, q_{n}\right)$ e $p=\left(p_{1}, p_{2}, \ldots, p_{n}\right)$, respectivamente.

Definição 1. Seja $U$ subconjunto aberto do espaço de fase e seja $\mathcal{F}$ o anel de todas as funções $f: U \rightarrow \mathbb{R}$ de classe $C^{\infty}$. O operador de Lie gerado por $W$ é a aplicação

$$
f \rightarrow \mathcal{L}_{W} f=\{f, W\}
$$

onde $\{f, W\}$ é o Colchete de Poisson das funções $f$ e $W \in \mathcal{F}$, dado por

$$
\{f, W\}=\sum_{i=1}^{N}\left(\frac{\partial f}{\partial q_{i}} \frac{\partial W}{\partial p_{i}}-\frac{\partial W}{\partial q_{i}} \frac{\partial f}{\partial p_{i}}\right) .
$$

O operador (3.1) tem as seguintes propriedades:

$$
\begin{aligned}
\mathcal{L}_{W}(f+g) & =\mathcal{L}_{W} f+\mathcal{L}_{W} g \\
\mathcal{L}_{W}(f g) & =f \mathcal{L}_{W} g+g \mathcal{L}_{W} f \\
\mathcal{L}_{W} c & =0 \\
\mathcal{L}_{W}\{f, g\} & =\left\{\mathcal{L}_{W} f, g\right\}+\left\{f, \mathcal{L}_{W} g\right\}
\end{aligned}
$$


para quaisquer que sejam $f, g \in \mathcal{F}$ e toda constante $c$.

Em relação a operação de composição, pode-se mostrar que $\mathcal{L}$ satisfaz:

$$
\mathcal{L}_{W}^{n} f=\mathcal{L}_{W}\left(\mathcal{L}_{W}^{n-1}(f)\right)
$$

De forma geral, de (3.3) e (3.4) podemos mostrar que :

$$
\begin{aligned}
\mathcal{L}_{W}^{n}(f+g) & =\mathcal{L}_{W}^{n}(f)+\mathcal{L}_{W}^{n}(g) \\
\mathcal{L}_{W}^{n}(f g) & =\sum_{k=0}^{n}\left(\begin{array}{l}
n \\
k
\end{array}\right) \mathcal{L}_{W}^{k} f \cdot \mathcal{L}_{W}^{n-k} g \\
\mathcal{L}_{W}^{n}\{f, g\} & =\sum_{k=0}^{n}\left(\begin{array}{l}
n \\
k
\end{array}\right)\left\{\mathcal{L}_{W}^{k} f, \mathcal{L}_{W}^{n-k} g\right\}
\end{aligned}
$$

Devemos notar que o operador de Lie é homogênio de grau $n$ em $W$, ou seja

$$
\mathcal{L}_{k W}^{n} f=k^{n} \mathcal{L}_{W} f, \quad \forall k \in \mathbb{R}
$$

\subsection{Séries de Lie}

Definição 2. Pra cada $f \in \mathcal{F}$ e cada ponto $(q, p) \in U$, e dado um gerador de Lie $W \in U$ de clase $C^{\infty}$, a aplicação

$$
f \mapsto E_{W} f=\sum_{k=0}^{\infty} \frac{\lambda^{k}}{k !} \mathcal{L}_{W}^{k} f
$$

é a série de Lie da função $f$, gerada por $W$.

De (3.5) podemos demonstrar que a aplicação (3.8) satisfaz as seguintes propiedades:

$$
\begin{gathered}
E_{W}(f+g)=E_{W} f+E_{W} g \\
E_{W}(f g)=E_{W} f \cdot E_{W} g \\
E_{W} c=c
\end{gathered}
$$

Afirmação 1. A transformação $(q, p) \Rightarrow\left(E_{W} q, E_{W} p\right)$ é canônica.

A demonstração desta afirmação é baseada na invariância dos colchetes de Poisson em relação a tranformações canônicas(ver Ferraz-Mello (2007)) 


\subsection{Teoria de Perturbações com Séries de Lie}

Consideremos Hamiltoniano independente do tempo

$$
H=H_{0}(\mathbf{J})+\epsilon R(\boldsymbol{\theta}, \mathbf{J})
$$

onde $\boldsymbol{\theta}=\left(\theta_{1}, \theta_{2}, \ldots, \theta_{N}\right), \mathbf{J}=\left(J_{1}, J_{2}, \cdots, J_{N}\right)$ são as variáveis ação-angulos.

De (3.10) temos o sistema de equaçõe canônicas dados por

$$
\begin{aligned}
\frac{d \theta_{i}}{d t} & =\frac{\partial H}{\partial J_{i}} \\
\frac{d J_{i}}{d t} & =-\frac{\partial H}{\partial \theta_{i}}
\end{aligned}
$$

Agora consideremos a transformação $(\boldsymbol{\theta}, \mathbf{J}) \Rightarrow\left(\boldsymbol{\theta}^{*}, \mathbf{J}^{*}\right)$, definida pela equação

$$
\phi(\boldsymbol{\theta}, \mathbf{J})=E_{W^{*}} \phi\left(\boldsymbol{\theta}^{*}, \mathbf{J}^{*}\right)=\sum_{k=0}^{\infty} \frac{1}{k !} \mathcal{L}_{W^{*}}^{k} \phi\left(\boldsymbol{\theta}^{*}, \mathbf{J}^{*}\right)
$$

onde $W^{*}=W\left(\boldsymbol{\theta}^{*}, \mathbf{J}\right)$.

Note que (3.13) correseponde a definição (3.8) fixando-se o parâmetro em $\lambda=1$.

Usando transformação (3.13) obtemos um novo Hamiltoniano dado por

$$
H(\boldsymbol{\theta}, \mathbf{J})=E_{W^{*}} H\left(\boldsymbol{\theta}^{*}, \mathbf{J}^{*}\right)=\sum_{k=0}^{\infty} \frac{1}{k !} \mathcal{L}_{W^{*}}^{k} H\left(\boldsymbol{\theta}^{*}, \mathbf{J}^{*}\right)
$$

Como a aplicação preserva o Hamiltoniano, então podemos escrever

$$
H^{*}\left(\boldsymbol{\theta}^{*}, \mathbf{J}^{*}\right)=H(\boldsymbol{\theta}, \mathbf{J}) .
$$

De (3.14) e (3.15), temos que

$$
\begin{gathered}
H^{*}\left(\boldsymbol{\theta}^{*}, \mathbf{J}^{*}\right)=E_{W^{*}} H\left(\boldsymbol{\theta}^{*}, \mathbf{J}^{*}\right) \\
H=H_{0}+\epsilon H_{1}+\epsilon^{2} H_{2}+\cdots \\
H^{*}=H_{0}^{*}+\epsilon H_{1}^{*}+\epsilon^{2} H_{2}^{*}+\cdots \\
W^{*}=\epsilon W_{1}^{*}+\epsilon^{2} W_{2}^{*}+\cdots
\end{gathered}
$$

Expandindo $H(\boldsymbol{\theta}, \mathbf{J})$ em séries de Lie e usando (3.17), obtemos equações 


$$
\begin{aligned}
H_{0}^{*} & =H_{0} \\
H_{1}^{*} & =H_{1}+\left\{H_{0}, W_{1}^{*}\right\} \\
H_{2}^{*} & =H_{2}+\left\{H_{1}, W_{1}^{*}\right\}+\frac{1}{2}\left\{\left\{H_{0}, W_{1}^{*}\right\}, W_{1}^{*}\right\}+\left\{H_{0}, W_{2}^{*}\right\} \\
& \vdots \\
H_{n}^{*} & =H_{n}+\left\{H_{n-1}, W_{1}^{*}\right\}+\frac{1}{2}\left\{\left\{H_{n-2}, W_{1}^{*}\right\}, W_{1}^{*}\right\}+\cdots+\left\{H_{0}, W_{n}^{*}\right\}
\end{aligned}
$$

Observemos que nas equações (3.18) temos $H_{k}^{*}=H_{k}^{*}\left(\boldsymbol{\theta}^{*}, \mathbf{J}^{*}\right)$ e $H_{k}=H_{k}(\boldsymbol{\theta}, \mathbf{J})$

\subsection{A equação Homológica}

Das equações (3.18), note que para a $k$ - ésima equação $W_{k}^{*}$ aparece apenas no termo $\left\{H_{0}, W_{k}^{*}\right\}$ e $H_{k}^{*}$ aparece apenas do lado esquerdo; tods os $W_{j}^{*}$ e $H_{j}^{*}$, nas equações, são tais que para $j<k$. Para $K \geq 1$ temos a equação homológica:

$$
H_{k}^{*}=\Psi_{k}+\left\{H_{0}, W_{k}^{*}\right\}
$$

onde $\Psi_{k}\left(\boldsymbol{\theta}^{*}, \mathbf{J}^{*}\right)$ é conhecida quando temos resolvido $k$ prévias equações.

Note que podemos reescrever (3.19) como

$$
H_{k}^{*}=\Psi_{k}+\left\{H_{0}, W_{k}^{*}\right\}=\sum_{i=1}^{N}\left(\frac{\partial H_{0}}{\partial \theta_{i}} \frac{\partial W_{k}^{*}}{\partial J_{i}}-\frac{\partial W_{k}^{*}}{\partial \theta_{i}} \frac{\partial H_{0}}{\partial J_{i}}\right)
$$

Observe também que se

$$
H_{0}=H_{0}(\mathbf{J}), \quad \mathbf{J}=\left(J_{1}, J_{2}, \cdots, J_{\mu}\right) \text { e } \mu \leq N
$$

então a equação (3.19) torna-se

$$
\sum_{\mu=1}^{M} \nu_{\mu}^{*} \frac{\partial W_{k}^{*}}{\partial \theta_{\mu}^{*}}=\Psi_{k}-H_{k}^{*}
$$

onde

$$
\nu_{\mu}^{*}=\frac{\partial H_{0}}{\partial J_{\mu}^{*}}
$$




\subsubsection{Regra da Média}

Note que para resolvermos a equação (3.19) precisamos conhecer umas das duas funções afin de evitarmos uma indeterminação. Para isto devemos ter uma maneira de escolhermos as funções indeterminadas $H_{k}^{*}$, esta maneira é definida por (3.24) para maiores detalhes ver Ferraz-Mello (2007). É importante notar que a equação homológica obtida com a Teoria de Lie coincide, ao menos na forma, com aquelas obtidas com as teorias de Poincaré e von Zeipel. Assim, procedemos de forma análoga para encontrarmos a solução da equação $(3.22)$.

\section{Definição 3.}

$$
H_{k}^{*}=\bar{\Psi}_{k}=\left(\frac{1}{2 \pi}\right)^{M} \int_{0}^{2 \pi} \cdots \int_{0}^{2 \pi} \Psi_{k} d \theta_{1}^{*} \cdots d \theta_{M}^{*} .
$$

Devemos observar que a média é feita em relação apenas aos ângulos cuja frequência associada $\nu_{\mu}^{*}$ é diferente de zero. Além disso, como $\Psi$ é uma função multiperiódica ela pode ser dividida em Parte secular, Parte de longo período e parte de curto período. Indicaremos estas partes por $S, L P$ e $S P$, respectivamente, mantendo a notação em inglês. Com isso, podemos reescrever (3.24) e (3.22) como

$$
H_{k}^{*}=\Psi_{k(S)}+\Psi_{k(L P)}
$$

$\mathrm{e}$

$$
\sum_{\mu=1}^{M} \nu_{\mu}^{*} \frac{\partial W_{k}^{*}}{\partial \theta_{\mu}^{*}}=\Psi_{k(S P)} .
$$

Note que o Hamiltoniano transformado $H^{*}$ não depende dos ângulos de curto-período, ou seja, de $\theta_{\nu}^{*}$ para $\nu=1, \cdots, M$. Isto implica que reduzimos o sistema original a $N-M$ graus de liberdade e o novo Hamiltoniano pode ser escrito como

$$
H^{*}=H_{0}\left(\mathbf{J}^{*}\right)+\sum_{k \geq 1} H_{k}^{*}\left(\boldsymbol{\theta}^{*}, \mathbf{J}^{*}\right), \quad \boldsymbol{\theta}^{*} \in \mathbb{T}^{N-M}, \mathbf{J}^{*} \in \mathbb{R}^{N}
$$

\subsection{Solução da Equação Homológica}

Por simplicidade de notação suponhamos que temos o seguinte Hamiltoniano

$$
H=H_{0}+\epsilon H_{1}
$$


Escrito nas variáveis não-singulares, onde $H_{0}$ é completamente integrável. A perturbação é dada por $H_{1}$, com $\epsilon$ pequeno.

Para o Hamiltoniano (3.28) ao aplicarmos a Teoria de Lie, até segunda ordem em $\epsilon$, obtemos:

$$
\begin{aligned}
H_{0}^{*} & =H_{0} \\
H_{1}^{*} & =H_{1}+\left\{H_{0}, W_{1}^{*}\right\} \\
H_{2}^{*} & =H_{2}+\left\{H_{1}, W_{1}^{*}\right\}+\frac{1}{2}\left\{\left\{H_{0}, W_{1}^{*}\right\}, W_{1}^{*}\right\} \\
& =H_{2}+\frac{1}{2}\left\{H_{1}+H_{1}^{*}, W_{1}^{*}\right\}
\end{aligned}
$$

A função geradora $W_{1}$ é obtida resolvendo a equação homológica,

$$
\sum_{\mu=1}^{M} \nu_{\mu}^{*} \frac{\partial W_{1}^{*}}{\partial \theta_{\mu}}=H_{1}-\overline{H_{1}}
$$

É importante lembrarmos que o termo do lado direito da equação (3.33) é uma série de Fourier, de modo que podemos resolver a equação integrando termo a termo. Assim, se $s(\boldsymbol{\theta})=H_{1}-\overline{H_{1}}$ e tem a forma

$$
s(\boldsymbol{\theta})=\sum_{\mathbf{K}} s_{\mathbf{k}} \exp (i \mathbf{k} \cdot \boldsymbol{\theta})
$$

onde $i=\sqrt{-1}, \mathbf{k}=\left(k_{1}, k_{2}, \ldots, k_{M}\right), \boldsymbol{\theta}=\left(\theta_{1}, \cdots, \theta_{M}\right)$ e $\mathbf{k} \cdot \boldsymbol{\theta}$ representa o produto escalar, então uma solução para a equação (3.33) tem a seguinte forma

$$
W_{1}(\boldsymbol{\theta})=\sum \frac{s_{\mathbf{k}}}{i \mathbf{k} \cdot \Omega} \exp (i \mathbf{k} \cdot \boldsymbol{\theta}), \quad \Omega=\left(\nu_{1}, \nu_{2}, \ldots, \nu_{M}\right)
$$

\subsection{Cálculo do Hamiltoniano Médio Na Vizinhança de Ressonâncias de Três Corpos}

Consideremos o Hamiltoniano (3.28) com $H_{0}$ e $H_{1}$ dados por (2.36) e (2.38), respectivamente. Notemos que cada argumeto da função perturbadora está relacionado com as longitude de apenas dois corpos diferentes, excluindo o terceiro. Portanto, a média será dada por

$$
\overline{H_{1}}=\frac{1}{(2 \pi)^{2}}\left(\int_{0}^{2 \pi} \int_{0}^{2 \pi} H_{1} d \lambda_{1} d \lambda_{2}+\int_{0}^{2 \pi} \int_{0}^{2 \pi} H_{1} d \lambda_{2} d \lambda_{3}+\int_{0}^{2 \pi} \int_{0}^{2 \pi} H_{1} d \lambda_{1} d \lambda_{3}\right)
$$




\subsubsection{Origem dos Termos que Envolvem Três Longitudes}

Agora vamos detalhar o procedimento feito para encontrarmos os termos em que aparecem as longitudes de três corpos. Observemos que a nossa função geratriz tem a forma:

$$
W_{1}^{*}=\tilde{W}_{1}+\tilde{W}_{2}+\cdots+\tilde{W}_{37}
$$

onde cada $\tilde{W}_{i}$ é "solução" da equação Homológica para cada $s_{i}$, e os $s_{i}^{\prime} s$ são os termos da série (3.34). O que precisamos calcular são os colchetes de Poissson da forma $\left\{H_{1}+\overline{H_{1}}, W_{1}^{*}\right\}$.

Sejam $F_{i}^{\prime} s$ os termos da soma $H_{1}+\overline{H_{1}}$, então vamos calcular os colchetes $\left\{F_{i}, \tilde{W}_{i}\right\}$. Como exemplo consideremos $F_{15}$ e $W_{15}$ dados por

$$
\begin{gathered}
F_{15}=\frac{24.72 G^{2} M_{3} \beta_{3}^{2}\left(2 P_{2}-\frac{P_{2}}{\Lambda_{2}}\right)^{2} \cos \left(4 \lambda_{2}-8 \lambda_{3}+4 \varpi_{2}\right)}{\Lambda_{2}^{2} \Lambda_{3}^{2}} \\
\tilde{W}_{15}=\frac{G^{2} M_{2} P_{2} \beta_{2}^{2}\left(0.716-1.43 \Lambda_{2}\right) \sqrt{\frac{P_{1}\left(2 \Lambda_{1}-P_{1}\right)}{\Lambda_{1}}} \sqrt{\frac{P_{2}\left(2 \Lambda_{2}-P_{2}\right)}{\Lambda_{2}}} \sin \left(\lambda_{1}-3 \lambda_{2}-\varpi_{1}+3 \varpi_{2}\right)}{\sqrt{\Lambda_{1} \Lambda_{2}^{9 / 2}\left(\nu_{1}-3 \nu_{2}\right)}}
\end{gathered}
$$

Após realizar alguma simplificações trigonométricas temos

$$
\begin{aligned}
\left\{F_{15}, \tilde{W}_{15}\right\} & =\frac{G^{4} M_{2} M_{3} \beta_{2}^{2} \beta_{3}^{2} \sqrt{\frac{P_{1}\left(2 \Lambda_{1}-P_{1}\right)}{\Lambda_{1}}} \sqrt{\frac{P_{2}\left(2 \Lambda_{2}-P_{2}\right)}{\Lambda_{2}}}}{\sqrt{\Lambda_{1}} \Lambda_{2}^{19 / 2} \Lambda_{3}^{2}\left(2 \Lambda_{2}-1\right)\left(\nu_{1}-3 \nu_{2}\right)} \\
& \times \Lambda_{2}\left(1700.52 P_{2}\left(\Lambda_{2}-0.50\right)\left(\Lambda_{2}-0.49\right)\left(\Lambda_{2}^{2}-\Lambda_{2}+0.25\right)\right. \\
& \left.-1133.52 P_{2}\left(\Lambda_{2}-0.50\right)\left(\Lambda_{2}-0.49\right)\left(\left(\Lambda_{2}^{2}-\Lambda_{2}+0.25\right)\right)\right) \\
& \times \cos \left(\lambda_{1}-7 \lambda_{2}+8 \lambda_{3}-\varpi_{1}-\varpi_{2}\right) \\
& +\frac{2833.52 G^{4} P_{2}^{3} M_{2} M_{3} \beta_{2}^{2} \beta_{3}^{2}}{\sqrt{\Lambda_{1}} \Lambda_{2}^{19 / 2} \Lambda_{3}^{2}\left(2 \Lambda_{2}-1\right)\left(\nu_{1}-3 \nu_{2}\right)}\left(\Lambda_{2}-0.8\right)\left(\Lambda_{2}-0.50\right) \\
& \times \sqrt{\frac{P_{1}\left(2 \Lambda_{1}-P_{1}\right)}{\Lambda_{1}}} \sqrt{\frac{P_{2}\left(2 \Lambda_{1}-P_{2}\right)}{\Lambda_{2}}} \cos \left(\lambda_{1}+\lambda_{2}-8 \lambda_{3}-\varpi_{1}+7 \varpi_{2}\right)
\end{aligned}
$$

O exemplo acima mostra, de forma geral, como foram calculados os termos que envolvem as longitudes médias de três corpos diferentes. 
Capítulo 4

\section{Resultados da Aplicação da Teoria de Lie ao Sistema}

\section{GJ581}

Neste capítulo mostramos, parcialmente, as ressonâncias de três corpos obtidas com a aplicação da teoria de Lie para perturbações. Damos também, parcialmente, os coeficientes relativos à ressonância $\lambda_{1}-2 \lambda_{2}-2 \lambda_{3}$.

\subsection{Ressonâncias Obtidas Utilizando a Teoria de Lie}

Na tabela (4.1) mostramos algumas ressonâncias obtidas usando a teoria de Lie, com os respectivos semieixos dentro da zona habitável. Devemos notar que ao aplicarmos o método descrito no capítulo (3), obtemos muitas ressonâncias cujo semieixo correspondente está fora da zona habitável, além disso muitas ressonâncias de altas ordens também são obtidas, como por exemplo as ressonâncias $\lambda_{1}+4 \lambda_{2}-13 \lambda_{3}, \lambda_{1}+2 \lambda_{2}-9 \lambda_{3}$ e $\lambda_{1}+3 \lambda_{2}-11 \lambda_{3}$. Nosso objetivo será aplicarmos a teoria de Chirikov descrita no capítulo (6) à ressonância $\lambda_{1}-2 \lambda_{2}-2 \lambda_{3}$. Esta ressonância foi escolhida pois ela está "isolada" de outras ressonâncias como mostra a figura (2.2).

\subsubsection{Coeficientes dos Multipletos da Ressonância $\lambda_{1}-2 \lambda_{2}-2 \lambda_{3}$}

Aqui mostraremos, parcialmente, os coeficientes correspondentes aos mutipletos da ressonância $\lambda_{1}-2 \lambda_{2}-2 \lambda_{3}$. No que segue, iremos associar a cada multipleto uma $\left(k_{4}, k_{5}, k_{6}\right) \in$ $\mathbb{Z}^{3}$, onde $k_{4}, k_{5}$ e $k_{6}$ são os coeficientes de $\varpi_{1}, \varpi_{2}$ e $\varpi_{3}$, respectivamente. Como exemplo, o coeficientes correspondente ao multipleto 1 da tabela (4.2) cujo argumento é $\lambda_{1}-2 \lambda_{2}-2 \lambda_{3}+3 \varpi_{1}$, será representado pela tripla $(3,0,0)$. Cada coeficiente será repre- 


\begin{tabular}{|c|c|c|c|}
\hline Número & Argumento & ordem & semi-eixo[UA] \\
\hline \hline 1 & $3 \lambda_{1}-9 \lambda_{2}+6 \lambda_{3}$ & 0 & 0.1220 \\
\hline 2 & $\lambda_{1}-6 \lambda_{2}+6 \lambda_{3}$ & 1 & 0.1442 \\
\hline 3 & $\lambda_{1}-4 \lambda_{2}+2 \lambda_{3}$ & 1 & 0.1478 \\
\hline 4 & $\lambda_{1}-8 \lambda_{2}+6 \lambda_{3}$ & 1 & 0.1746 \\
\hline 5 & $3 \lambda_{1}-7 \lambda_{2}+2 \lambda_{3}$ & 2 & 0.1183 \\
\hline 6 & $\lambda_{1}-2 \lambda_{2}-2 \lambda_{3}$ & 3 & 0.1603 \\
\hline 7 & $\lambda_{1}-9 \lambda_{2}+8 \lambda_{3}$ & 0 & 0.1693 \\
\hline 8 & $2 \lambda_{1}-6 \lambda_{2}+5 \lambda_{3}$ & 1 & 0.1166 \\
\hline 9 & $\lambda_{1}-7 \lambda_{2}+8 \lambda_{3}$ & 2 & 0.1432 \\
\hline 10 & $\lambda_{1}-3 \lambda_{2}+\lambda_{3}$ & 1 & 0.1349 \\
\hline
\end{tabular}

Tabela 4.1 - Principais ressonâncias no intervalo considerado.

sentado como a soma $\sum_{i} C_{i}^{j}$, o índice $i$ correspondendo ao respectivo multipleto e o índice $j$ correspondendo a $j$ - ésima parte pela qual ele é formado. Por exemplo, o coeficiente $C_{1}$ correspondente ao multipleto $(3,0,0)$ é dado pela soma

$$
C_{1}=C_{1}^{1}+C_{1}^{2}+C_{1}^{3}+C_{1}^{4}+C_{1}^{5}
$$

onde cada $C_{i}^{j}, j=1, \ldots, 5$, são dados por $(4.2), \ldots,(4.6)$, respectivamente. Por questões práticas, para os outros coeficientes dos multipletos, daremos apenas uma pequena parte de seus coeficientes. Para os multipletos $(2,1,0)$ o respectivo coeficiente é dado por $C_{2}=$ $C_{2}^{1}+C_{2}^{2}$, onde $C_{1}^{1}$ e $C_{2}^{2}$ são dados por (4.7) e (4.8), respectivamente. De forma geral, os outros coeficientes são dados por $C_{i}=C_{i}^{1}+C_{i}^{2}$, onde os $C_{i}^{1}$ e $C_{i}^{2}$ são dados pelas expressões $(4.7), \ldots,(4.36)$. 


\begin{tabular}{|c|c|}
\hline Número & Multipleto \\
\hline \hline 1 & $\lambda_{1}-2 \lambda_{2}-2 \lambda_{3}+3 \varpi_{1}$ \\
\hline 2 & $\lambda_{1}-2 \lambda_{2}-2 \lambda_{3}+2 \varpi_{1}+\varpi_{2}$ \\
\hline 3 & $\lambda_{1}-2 \lambda_{2}-2 \lambda_{3}+\varpi_{1}+2 \varpi_{2}$ \\
\hline 4 & $\lambda_{1}-2 \lambda_{2}-2 \lambda_{3}-\varpi_{1}+4 \varpi_{2}$ \\
\hline 5 & $\lambda_{1}-2 \lambda_{2}-2 \lambda_{3}+3 \varpi_{1}+\varpi_{2}-\varpi_{3}$ \\
\hline 6 & $\lambda_{1}-2 \lambda_{2}-2 \lambda_{3}+2 \varpi_{1}+2 \varpi_{2}-\varpi_{3}$ \\
\hline 7 & $\lambda_{1}-2 \lambda_{2}-2 \lambda_{3}+\varpi_{1}+3 \varpi_{2}-\varpi_{3}$ \\
\hline 8 & $\lambda_{1}-2 \lambda_{2}-2 \lambda_{3}-\varpi_{1}+5 \varpi_{2}-\varpi_{3}$ \\
\hline 9 & $\lambda_{1}-2 \lambda_{2}-2 \lambda_{3}+2 \varpi_{1}+\varpi_{3}$ \\
\hline 10 & $\lambda_{1}-2 \lambda_{2}-2 \lambda_{3}+3 \varpi_{1}-\varpi_{2}+\varpi_{3}$ \\
\hline 11 & $\lambda_{1}-2 \lambda_{2}-2 \lambda_{3}+\varpi_{1}+\varpi_{2}+\varpi_{3}$ \\
\hline 12 & $\lambda_{1}-2 \lambda_{2}-2 \lambda_{3}-\varpi_{1}+3 \varpi_{2}+\varpi_{3}$ \\
\hline 13 & $\lambda_{1}-2 \lambda_{2}-2 \lambda_{3}+\varpi_{1}+2 \varpi_{3}$ \\
\hline 14 & $\lambda_{1}-2 \lambda_{2}-2 \lambda_{3}+3 \varpi_{1}-2 \varpi_{2}+2 \varpi_{3}$ \\
\hline 15 & $\lambda_{1}-2 \lambda_{2}-2 \lambda_{3}+2 \varpi_{1}-\varpi_{2}+2 \varpi_{3}$ \\
\hline 16 & $\lambda_{1}-2 \lambda_{2}-2 \lambda_{3}-\varpi_{1}+2 \varpi_{2}+2 \varpi_{3}$ \\
\hline
\end{tabular}

Tabela 4.2 - Multipletos da ressonância $\lambda_{1}-2 \lambda_{2}-2 \lambda_{3}$.

$$
\begin{aligned}
C_{1}^{1} & =\frac{8.76 \beta_{2}^{2} \beta_{3}^{2} G^{4} M_{2} M_{3} P_{1} P_{2}^{2} P_{3}^{2} \sqrt{-\frac{P_{1}\left(P_{1}-2 \Lambda_{1}\right)}{\Lambda_{1}}}}{\Lambda_{1}^{3 / 2} \Lambda_{2}^{5} \Lambda_{3}^{4}\left(\nu_{1}-3 \nu_{2}\right)}-\frac{4.38324 \beta_{2}^{2} \beta_{3}^{2} G^{4} M_{2} M_{3} P_{1}^{2} P_{2}^{2} P_{3}^{2} \sqrt{-\frac{P_{1}\left(P_{1}-2 \Lambda_{1}\right)}{\Lambda_{1}}}}{\Lambda_{1}^{5 / 2} \Lambda_{2}^{5} \Lambda_{3}^{4}\left(\nu_{1}-3 \nu_{2}\right)} \\
& +\frac{7.3054 \beta_{2}^{2} \beta_{3}^{2} G^{4} M_{2} M_{3} P_{1}^{2} P_{2} P_{3}^{2} \sqrt{-\frac{P_{1}\left(P_{1}-2 \Lambda_{1}\right)}{\Lambda_{1}}}}{\Lambda_{1}^{5 / 2} \Lambda_{2}^{4} \Lambda_{3}^{4}\left(\nu_{1}-3 \nu_{2}\right)}-\frac{14.6108 \beta_{2}^{2} \beta_{3}^{2} G^{4} M_{2} M_{3} P_{1} P_{2} P_{3}^{2} \sqrt{-\frac{P_{1}\left(P_{1}-2 \Lambda_{1}\right)}{\Lambda_{1}}}}{\Lambda_{1}^{3 / 2} \Lambda_{2}^{4} \Lambda_{3}^{4}\left(\nu_{1}-3 \nu_{2}\right)} \\
& +\frac{2.92 \beta_{2}^{2} \beta_{3}^{2} G^{4} M_{2} M_{3} P_{1} P_{3}^{2} \sqrt{-\frac{P_{1}\left(P_{1}-2 \Lambda_{1}\right)}{\Lambda_{1}}}}{\Lambda_{1}^{3 / 2} \Lambda_{2}^{3} \Lambda_{3}^{4}\left(\nu_{1}-3 \nu_{2}\right)}-\frac{1.46108 \beta_{2}^{2} \beta_{3}^{2} G^{4} M_{2} M_{3} P_{1}^{2} P_{3}^{2} \sqrt{-\frac{P_{1}\left(P_{1}-2 \Lambda_{1}\right)}{\Lambda_{1}}}}{\Lambda_{1}^{5 / 2} \Lambda_{2}^{3} \Lambda_{3}^{4}\left(\nu_{1}-3 \nu_{2}\right)} \\
+ & \frac{8.76 \beta_{2}^{2} \beta_{3}^{2} G^{4} M_{2} M_{3} P_{1}^{2} P_{2}^{2} P_{3} \sqrt{-\frac{P_{1}\left(P_{1}-2 \Lambda_{1}\right)}{\Lambda_{1}}}}{\Lambda_{1}^{5 / 2} \Lambda_{2}^{5} \Lambda_{3}^{3}\left(\nu_{1}-3 \nu_{2}\right)}-\frac{17.533 \beta_{2}^{2} \beta_{3}^{2} G^{4} M_{2} M_{3} P_{1} P_{2}^{2} P_{3} \sqrt{-\frac{P_{1}\left(P_{1}-2 \Lambda_{1}\right)}{\Lambda_{1}}}}{\Lambda_{1}^{3 / 2} \Lambda_{2}^{5} \Lambda_{3}^{3}\left(\nu_{1}-3 \nu_{2}\right)}
\end{aligned}
$$




$$
\begin{aligned}
C_{1}^{2} & =\frac{29.2216 \beta_{2}^{2} \beta_{3}^{2} G^{4} M_{2} M_{3} P_{1} P_{2} P_{3} \sqrt{-\frac{P_{1}\left(P_{1}-2 \Lambda_{1}\right)}{\Lambda_{1}}}}{\Lambda_{1}^{3 / 2} \Lambda_{2}^{4} \Lambda_{3}^{3}\left(\nu_{1}-3 \nu_{2}\right)}-\frac{14.6108 \beta_{2}^{2} \beta_{3}^{2} G^{4} M_{2} M_{3} P_{1}^{2} P_{2} P_{3} \sqrt{-\frac{P_{1}\left(P_{1}-2 \Lambda_{1}\right)}{\Lambda_{1}}}}{\Lambda_{1}^{5 / 2} \Lambda_{2}^{4} \Lambda_{3}^{3}\left(\nu_{1}-3 \nu_{2}\right)} \\
& +\frac{2.92216 \beta_{2}^{2} \beta_{3}^{2} G^{4} M_{2} M_{3} P_{1}^{2} P_{3} \sqrt{-\frac{P_{1}\left(P_{1}-2 \Lambda_{1}\right)}{\Lambda_{1}}}}{\Lambda_{1}^{5 / 2} \Lambda_{2}^{3} \Lambda_{3}^{3}\left(\nu_{1}-3 \nu_{2}\right)}-\frac{5.84432 \beta_{2}^{2} \beta_{3}^{2} G^{4} M_{2} M_{3} P_{1} P_{3} \sqrt{-\frac{P_{1}\left(P_{1}-2 \Lambda_{1}\right)}{\Lambda_{1}}}}{\Lambda_{1}^{3 / 2} \Lambda_{2}^{3} \Lambda_{3}^{3}\left(\nu_{1}-3 \nu_{2}\right)}
\end{aligned}
$$$$
+\frac{2.703 \beta_{2}^{2} \beta_{3}^{2} G^{4} M_{2} M_{3} P_{1} P_{2}^{4} \sqrt{-\frac{P_{1}\left(P_{1}-2 \Lambda_{1}\right)}{\Lambda_{1}}}}{\Lambda_{1}^{3 / 2} \Lambda_{2}^{7} \Lambda_{3}^{2}\left(\nu_{1}-3 \nu_{2}\right)}-\frac{1.35186 \beta_{2}^{2} \beta_{3}^{2} G^{4} M_{2} M_{3} P_{1}^{2} P_{2}^{4} \sqrt{-\frac{P_{1}\left(P_{1}-2 \Lambda_{1}\right)}{\Lambda_{1}}}}{\Lambda_{1}^{5 / 2} \Lambda_{2}^{7} \Lambda_{3}^{2}\left(\nu_{1}-3 \nu_{2}\right)}
$$$$
+\frac{4.731 \beta_{2}^{2} \beta_{3}^{2} G^{4} M_{2} M_{3} P_{1}^{2} P_{2}^{3} \sqrt{-\frac{P_{1}\left(P_{1}-2 \Lambda_{1}\right)}{\Lambda_{1}}}}{\Lambda_{1}^{5 / 2} \Lambda_{2}^{6} \Lambda_{3}^{2}\left(\nu_{1}-3 \nu_{2}\right)}-\frac{9.46305 \beta_{2}^{2} \beta_{3}^{2} G^{4} M_{2} M_{3} P_{1} P_{2}^{3} \sqrt{-\frac{P_{1}\left(P_{1}-2 \Lambda_{1}\right)}{\Lambda_{1}}}}{\Lambda_{1}^{3 / 2} \Lambda_{2}^{6} \Lambda_{3}^{2}\left(\nu_{1}-3 \nu_{2}\right)}
$$

$$
\begin{aligned}
C_{1}^{3} & =\frac{2.6012 \beta_{2}^{2} \beta_{3}^{2} G^{4} M_{2} M_{3} P_{1} P_{2}^{2} \sqrt{-\frac{P_{1}\left(P_{1}-2 \Lambda_{1}\right)}{\Lambda_{1}}}}{\Lambda_{1}^{3 / 2} \Lambda_{2}^{5} \Lambda_{3}^{2}\left(\nu_{1}-3 \nu_{2}\right)}-\frac{1.30 \beta_{2}^{2} \beta_{3}^{2} G^{4} M_{2} M_{3} P_{1}^{2} P_{2}^{2} \sqrt{-\frac{P_{1}\left(P_{1}-2 \Lambda_{1}\right)}{\Lambda_{1}}}}{\Lambda_{1}^{5 / 2} \Lambda_{2}^{5} \Lambda_{3}^{2}\left(\nu_{1}-3 \nu_{2}\right)} \\
& +\frac{8.8829 \beta_{2}^{2} \beta_{3}^{2} G^{4} M_{2} M_{3} P_{1} P_{2} \sqrt{-\frac{P_{1}\left(P_{1}-2 \Lambda_{1}\right)}{\Lambda_{1}}}}{\Lambda_{1}^{3 / 2} \Lambda_{2}^{4} \Lambda_{3}^{2}\left(\nu_{1}-3 \nu_{2}\right)}-\frac{4.44145 \beta_{2}^{2} \beta_{3}^{2} G^{4} M_{2} M_{3} P_{1}^{2} P_{2} \sqrt{-\frac{P_{1}\left(P_{1}-2 \Lambda_{1}\right)}{\Lambda_{1}}}}{\Lambda_{1}^{5 / 2} \Lambda_{2}^{4} \Lambda_{3}^{2}\left(\nu_{1}-3 \nu_{2}\right)}
\end{aligned}
$$

$$
+\frac{1.06 \beta_{2}^{2} \beta_{3}^{2} G^{4} M_{2} M_{3} P_{1}^{2} \sqrt{-\frac{P_{1}\left(P_{1}-2 \Lambda_{1}\right)}{\Lambda_{1}}}}{\Lambda_{1}^{5 / 2} \Lambda_{2}^{3} \Lambda_{3}^{2}\left(\nu_{1}-3 \nu_{2}\right)}-\frac{2.13708 \beta_{2}^{2} \beta_{3}^{2} G^{4} M_{2} M_{3} P_{1} \sqrt{-\frac{P_{1}\left(P_{1}-2 \Lambda_{1}\right)}{\Lambda_{1}}}}{\Lambda_{1}^{3 / 2} \Lambda_{2}^{3} \Lambda_{3}^{2}\left(\nu_{1}-3 \nu_{2}\right)}
$$

$+\frac{4.38 \beta_{2}^{2} \beta_{3}^{2} G^{4} M_{2} M_{3} P_{1}^{2} P_{2}^{2} P_{3}^{2} \sqrt{-\frac{P_{1}\left(P_{1}-2 \Lambda_{1}\right)}{\Lambda_{1}}}}{\Lambda_{1}^{5 / 2} \Lambda_{2}^{5} \Lambda_{3}^{4}\left(\nu_{2}-2 \nu_{3}\right)}-\frac{8.76 \beta_{2}^{2} \beta_{3}^{2} G^{4} M_{2} M_{3} P_{1} P_{2}^{2} P_{3}^{2} \sqrt{-\frac{P_{1}\left(P_{1}-2 \Lambda_{1}\right)}{\Lambda_{1}}}}{\Lambda_{1}^{3 / 2} \Lambda_{2}^{5} \Lambda_{3}^{4}\left(\nu_{2}-2 \nu_{3}\right)}$ 


$$
\begin{aligned}
C_{1}^{5} & =\frac{5.84432 \beta_{2}^{2} \beta_{3}^{2} G^{4} M_{2} M_{3} P_{1} P_{3} \sqrt{-\frac{P_{1}\left(P_{1}-2 \Lambda_{1}\right)}{\Lambda_{1}}}}{\Lambda_{1}^{3 / 2} \Lambda_{2}^{3} \Lambda_{3}^{3}\left(\nu_{2}-2 \nu_{3}\right)}-\frac{2.92216 \beta_{2}^{2} \beta_{3}^{2} G^{4} M_{2} M_{3} P_{1}^{2} P_{3} \sqrt{-\frac{P_{1}\left(P_{1}-2 \Lambda_{1}\right)}{\Lambda_{1}}}}{\Lambda_{1}^{5 / 2} \Lambda_{2}^{3} \Lambda_{3}^{3}\left(\nu_{2}-2 \nu_{3}\right)} \\
& +\frac{1.35186 \beta_{2}^{2} \beta_{3}^{2} G^{4} M_{2} M_{3} P_{1}^{2} P_{2}^{4} \sqrt{-\frac{P_{1}\left(P_{1}-2 \Lambda_{1}\right)}{\Lambda_{1}}}}{\Lambda_{1}^{5 / 2} \Lambda_{2}^{7} \Lambda_{3}^{2}\left(\nu_{2}-2 \nu_{3}\right)}-\frac{2.70373 \beta_{2}^{2} \beta_{3}^{2} G^{4} M_{2} M_{3} P_{1} P_{2}^{4} \sqrt{-\frac{P_{1}\left(P_{1}-2 \Lambda_{1}\right)}{\Lambda_{1}}}}{\Lambda_{1}^{3 / 2} \Lambda_{2}^{7} \Lambda_{3}^{2}\left(\nu_{2}-2 \nu_{3}\right)} \\
& +\frac{9.46305 \beta_{2}^{2} \beta_{3}^{2} G^{4} M_{2} M_{3} P_{1} P_{2}^{3} \sqrt{-\frac{P_{1}\left(P_{1}-2 \Lambda_{1}\right)}{\Lambda_{1}}}}{\Lambda_{1}^{3 / 2} \Lambda_{2}^{6} \Lambda_{3}^{2}\left(\nu_{2}-2 \nu_{3}\right)}-\frac{4.73153 \beta_{2}^{2} \beta_{3}^{2} G^{4} M_{2} M_{3} P_{1}^{2} P_{2}^{3} \sqrt{-\frac{P_{1}\left(P_{1}-2 \Lambda_{1}\right)}{\Lambda_{1}}}}{\Lambda_{1}^{5 / 2} \Lambda_{2}^{6} \Lambda_{3}^{2}\left(\nu_{2}-2 \nu_{3}\right)} \\
+ & \frac{1.3006 \beta_{2}^{2} \beta_{3}^{2} G^{4} M_{2} M_{3} P_{1}^{2} P_{2}^{2} \sqrt{-\frac{P_{1}\left(P_{1}-2 \Lambda_{1}\right)}{\Lambda_{1}}}}{\Lambda_{1}^{5 / 2} \Lambda_{2}^{5} \Lambda_{3}^{2}\left(\nu_{2}-2 \nu_{3}\right)}-\frac{2.6012 \beta_{2}^{2} \beta_{3}^{2} G^{4} M_{2} M_{3} P_{1} P_{2}^{2} \sqrt{-\frac{P_{1}\left(P_{1}-2 \Lambda_{1}\right)}{\Lambda_{1}}}}{\Lambda_{1}^{3 / 2} \Lambda_{2}^{5} \Lambda_{3}^{2}\left(\nu_{2}-2 \nu_{3}\right)}
\end{aligned}
$$

$$
\begin{aligned}
C_{2}^{1} & =\frac{14.3914 \beta_{2}^{2} \beta_{3}^{2} G^{4} M_{2} M_{3} P_{2}^{5} P_{3}^{2} \sqrt{-\frac{P_{2}\left(P_{2}-2 \Lambda_{2}\right)}{\Lambda_{2}}}}{\Lambda_{2}^{15 / 2} \Lambda_{3}^{4}\left(\nu_{1}-3 \nu_{2}\right)\left(P_{2}-2 \Lambda_{2}\right)}-\frac{81.5512 \beta_{2}^{2} \beta_{3}^{2} G^{4} M_{2} M_{3} P_{2}^{4} P_{3}^{2} \sqrt{-\frac{P_{2}\left(P_{2}-2 \Lambda_{2}\right)}{\Lambda_{2}}}}{\Lambda_{2}^{13 / 2} \Lambda_{3}^{4}\left(\nu_{1}-3 \nu_{2}\right)\left(P_{2}-2 \Lambda_{2}\right)} \\
& +\frac{176.526 \beta_{2}^{2} \beta_{3}^{2} G^{4} M_{2} M_{3} P_{2}^{3} P_{3}^{2} \sqrt{-\frac{P_{2}\left(P_{2}-2 \Lambda_{2}\right)}{\Lambda_{2}}}}{\Lambda_{2}^{11 / 2} \Lambda_{3}^{4}\left(\nu_{1}-3 \nu_{2}\right)\left(P_{2}-2 \Lambda_{2}\right)}+\frac{22.3847 \beta_{2}^{2} \beta_{3}^{2} G^{4} M_{2} M_{3} P_{1}^{2} P_{2}^{3} P_{3}^{2} \sqrt{-\frac{P_{2}\left(P_{2}-2 \Lambda_{2}\right)}{\Lambda_{2}}}}{\Lambda_{1}^{2} \Lambda_{2}^{11 / 2} \Lambda_{3}^{4}\left(\nu_{1}-3 \nu_{2}\right)\left(P_{2}-2 \Lambda_{2}\right)}
\end{aligned}
$$

$-\frac{180.889 \beta_{2}^{2} \beta_{3}^{2} G^{4} M_{2} M_{3} P_{2}^{2} P_{3}^{2} \sqrt{-\frac{P_{2}\left(P_{2}-2 \Lambda_{2}\right)}{\Lambda_{2}}}}{\Lambda_{2}^{9 / 2} \Lambda_{3}^{4}\left(\nu_{1}-3 \nu_{2}\right)\left(P_{2}-2 \Lambda_{2}\right)}-\frac{44.7694 \beta_{2}^{2} \beta_{3}^{2} G^{4} M_{2} M_{3} P_{1} P_{2}^{3} P_{3}^{2} \sqrt{-\frac{P_{2}\left(P_{2}-2 \Lambda_{2}\right)}{\Lambda_{2}}}}{\Lambda_{1} \Lambda_{2}^{11 / 2} \Lambda_{3}^{4}\left(\nu_{1}-3 \nu_{2}\right)\left(P_{2}-2 \Lambda_{2}\right)}$

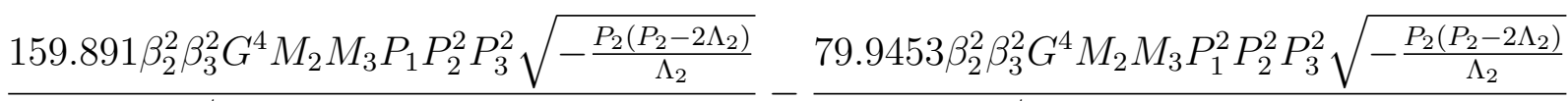

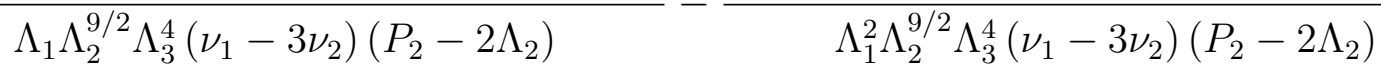

$$
\begin{aligned}
C_{2}^{2} & =\frac{82.5716 \beta_{2}^{2} \beta_{3}^{2} G^{4} M_{2} M_{3} P_{2} P_{3}^{2} \sqrt{-\frac{P_{2}\left(P_{2}-2 \Lambda_{2}\right)}{\Lambda_{2}}}}{\Lambda_{2}^{7 / 2} \Lambda_{3}^{4}\left(\nu_{1}-3 \nu_{2}\right)\left(P_{2}-2 \Lambda_{2}\right)}-\frac{2.91595 \beta_{2}^{2} \beta_{3}^{2} G^{4} M_{2} M_{3} P_{1}^{4} P_{2} P_{3}^{2} \sqrt{-\frac{P_{2}\left(P_{2}-2 \Lambda_{2}\right)}{\Lambda_{2}}}}{\Lambda_{1}^{4} \Lambda_{2}^{7 / 2} \Lambda_{3}^{4}\left(\nu_{1}-3 \nu_{2}\right)\left(P_{2}-2 \Lambda_{2}\right)} \\
& +\frac{68.3004 \beta_{2}^{2} \beta_{3}^{2} G^{4} M_{2} M_{3} P_{1}^{2} P_{2} P_{3}^{2} \sqrt{-\frac{P_{2}\left(P_{2}-2 \Lambda_{2}\right)}{\Lambda_{2}}}}{\Lambda_{1}^{2} \Lambda_{2}^{7 / 2} \Lambda_{3}^{4}\left(\nu_{1}-3 \nu_{2}\right)\left(P_{2}-2 \Lambda_{2}\right)}+\frac{11.6638 \beta_{2}^{2} \beta_{3}^{2} G^{4} M_{2} M_{3} P_{1}^{3} P_{2} P_{3}^{2} \sqrt{-\frac{P_{2}\left(P_{2}-2 \Lambda_{2}\right)}{\Lambda_{2}}}}{\Lambda_{1}^{3} \Lambda_{2}^{7 / 2} \Lambda_{3}^{4}\left(\nu_{1}-3 \nu_{2}\right)\left(P_{2}-2 \Lambda_{2}\right)}
\end{aligned}
$$

$-\frac{9.49781 \beta_{2}^{2} \beta_{3}^{2} G^{4} M_{2} M_{3} P_{3}^{2} \sqrt{-\frac{P_{2}\left(P_{2}-2 \Lambda_{2}\right)}{\Lambda_{2}}}}{\Lambda_{2}^{5 / 2} \Lambda_{3}^{4}\left(\nu_{1}-3 \nu_{2}\right)\left(P_{2}-2 \Lambda_{2}\right)}-\frac{159.928 \beta_{2}^{2} \beta_{3}^{2} G^{4} M_{2} M_{3} P_{1} P_{2} P_{3}^{2} \sqrt{-\frac{P_{2}\left(P_{2}-2 \Lambda_{2}\right)}{\Lambda_{2}}}}{\Lambda_{1} \Lambda_{2}^{7 / 2} \Lambda_{3}^{4}\left(\nu_{1}-3 \nu_{2}\right)\left(P_{2}-2 \Lambda_{2}\right)}$

$+\frac{4.08233 \beta_{2}^{2} \beta_{3}^{2} G^{4} M_{2} M_{3} P_{1}^{4} P_{3}^{2} \sqrt{-\frac{P_{2}\left(P_{2}-2 \Lambda_{2}\right)}{\Lambda_{2}}}}{\Lambda_{1}^{4} \Lambda_{2}^{5 / 2} \Lambda_{3}^{4}\left(\nu_{1}-3 \nu_{2}\right)\left(P_{2}-2 \Lambda_{2}\right)}-\frac{16.3293 \beta_{2}^{2} \beta_{3}^{2} G^{4} M_{2} M_{3} P_{1}^{3} P_{3}^{2} \sqrt{-\frac{P_{2}\left(P_{2}-2 \Lambda_{2}\right)}{\Lambda_{2}}}}{\Lambda_{1}^{3} \Lambda_{2}^{5 / 2} \Lambda_{3}^{4}\left(\nu_{1}-3 \nu_{2}\right)\left(P_{2}-2 \Lambda_{2}\right)}$ 


$$
\begin{aligned}
C_{3}^{1} & =\frac{119.896 \beta_{2}^{2} \beta_{3}^{2} G^{4} M_{2} M_{3} P_{2}^{3} P_{3}^{2} \sqrt{-\frac{P_{1}\left(P_{1}-2 \Lambda_{1}\right)}{\Lambda_{1}}}}{\sqrt{\Lambda_{1}} \Lambda_{2}^{6} \Lambda_{3}^{4}\left(\nu_{1}-3 \nu_{2}\right)}-\frac{34.2559 \beta_{2}^{2} \beta_{3}^{2} G^{4} M_{2} M_{3} P_{2}^{4} P_{3}^{2} \sqrt{-\frac{P_{1}\left(P_{1}-2 \Lambda_{1}\right)}{\Lambda_{1}}}}{\sqrt{\Lambda_{1}} \Lambda_{2}^{7} \Lambda_{3}^{4}\left(\nu_{1}-3 \nu_{2}\right)} \\
+ & \frac{6.86359 \beta_{2}^{2} \beta_{3}^{2} G^{4} M_{2} M_{3} P_{1} P_{2}^{2} P_{3}^{2} \sqrt{-\frac{P_{1}\left(P_{1}-2 \Lambda_{1}\right)}{\Lambda_{1}}}}{\Lambda_{1}^{3 / 2} \Lambda_{2}^{5} \Lambda_{3}^{4}\left(\nu_{1}-3 \nu_{2}\right)}-\frac{3.4318 \beta_{2}^{2} \beta_{3}^{2} G^{4} M_{2} M_{3} P_{1}^{2} P_{2}^{2} P_{3}^{2} \sqrt{-\frac{P_{1}\left(P_{1}-2 \Lambda_{1}\right)}{\Lambda_{1}}}}{\Lambda_{1}^{5 / 2} \Lambda_{2}^{5} \Lambda_{3}^{4}\left(\nu_{1}-3 \nu_{2}\right)} \\
+ & \frac{4.57573 \beta_{2}^{2} \beta_{3}^{2} G^{4} M_{2} M_{3} P_{1}^{2} P_{2} P_{3}^{2} \sqrt{-\frac{P_{1}\left(P_{1}-2 \Lambda_{1}\right)}{\Lambda_{1}}}}{\Lambda_{1}^{5 / 2} \Lambda_{2}^{4} \Lambda_{3}^{4}\left(\nu_{1}-3 \nu_{2}\right)}-\frac{126.383 \beta_{2}^{2} \beta_{3}^{2} G^{4} M_{2} M_{3} P_{2}^{2} P_{3}^{2} \sqrt{-\frac{P_{1}\left(P_{1}-2 \Lambda_{1}\right)}{\Lambda_{1}}}}{\sqrt{\Lambda_{1}} \Lambda_{2}^{5} \Lambda_{3}^{4}\left(\nu_{1}-3 \nu_{2}\right)} \\
+ & \frac{37.1967 \beta_{2}^{2} \beta_{3}^{2} G^{4} M_{2} M_{3} P_{2} P_{3}^{2} \sqrt{-\frac{P_{1}\left(P_{1}-2 \Lambda_{1}\right)}{\Lambda_{1}}}}{\sqrt{\Lambda_{1}} \Lambda_{2}^{4} \Lambda_{3}^{4}\left(\nu_{1}-3 \nu_{2}\right)}-\frac{9.15146 \beta_{2}^{2} \beta_{3}^{2} G^{4} M_{2} M_{3} P_{1} P_{2} P_{3}^{2} \sqrt{-\frac{P_{1}\left(P_{1}-2 \Lambda_{1}\right)}{\Lambda_{1}}}}{\Lambda_{1}^{3 / 2} \Lambda_{2}^{4} \Lambda_{3}^{4}\left(\nu_{1}-3 \nu_{2}\right)}
\end{aligned}
$$

$\begin{aligned} C_{3}^{2} & =\frac{68.5118 \beta_{2}^{2} \beta_{3}^{2} G^{4} M_{2} M_{3} P_{2}^{4} P_{3} \sqrt{-\frac{P_{1}\left(P_{1}-2 \Lambda_{1}\right)}{\Lambda_{1}}}}{\sqrt{\Lambda_{1}} \Lambda_{2}^{7} \Lambda_{3}^{3}\left(\nu_{1}-3 \nu_{2}\right)}-\frac{239.791 \beta_{2}^{2} \beta_{3}^{2} G^{4} M_{2} M_{3} P_{2}^{3} P_{3} \sqrt{-\frac{P_{1}\left(P_{1}-2 \Lambda_{1}\right)}{\Lambda_{1}}}}{\sqrt{\Lambda_{1}} \Lambda_{2}^{6} \Lambda_{3}^{3}\left(\nu_{1}-3 \nu_{2}\right)} \\ & +\frac{6.86 \beta_{2}^{2} \beta_{3}^{2} G^{4} M_{2} M_{3} P_{1}^{2} P_{2}^{2} P_{3} \sqrt{-\frac{P_{1}\left(P_{1}-2 \Lambda_{1}\right)}{\Lambda_{1}}}}{\Lambda_{1}^{5 / 2} \Lambda_{2}^{5} \Lambda_{3}^{3}\left(\nu_{1}-3 \nu_{2}\right)}-\frac{13.72 \beta_{2}^{2} \beta_{3}^{2} G^{4} M_{2} M_{3} P_{1} P_{2}^{2} P_{3} \sqrt{-\frac{P_{1}\left(P_{1}-2 \Lambda_{1}\right)}{\Lambda_{1}}}}{\Lambda_{1}^{3 / 2} \Lambda_{2}^{5} \Lambda_{3}^{3}\left(\nu_{1}-3 \nu_{2}\right)}\end{aligned}$

$+\frac{252.766 \beta_{2}^{2} \beta_{3}^{2} G^{4} M_{2} M_{3} P_{2}^{2} P_{3} \sqrt{-\frac{P_{1}\left(P_{1}-2 \Lambda_{1}\right)}{\Lambda_{1}}}}{\sqrt{\Lambda_{1}} \Lambda_{2}^{5} \Lambda_{3}^{3}\left(\nu_{1}-3 \nu_{2}\right)}-\frac{9.15146 \beta_{2}^{2} \beta_{3}^{2} G^{4} M_{2} M_{3} P_{1}^{2} P_{2} P_{3} \sqrt{-\frac{P_{1}\left(P_{1}-2 \Lambda_{1}\right)}{\Lambda_{1}}}}{\Lambda_{1}^{5 / 2} \Lambda_{2}^{4} \Lambda_{3}^{3}\left(\nu_{1}-3 \nu_{2}\right)}$

$+\frac{18.3029 \beta_{2}^{2} \beta_{3}^{2} G^{4} M_{2} M_{3} P_{1} P_{2} P_{3} \sqrt{-\frac{P_{1}\left(P_{1}-2 \Lambda_{1}\right)}{\Lambda_{1}}}}{\Lambda_{1}^{3 / 2} \Lambda_{2}^{4} \Lambda_{3}^{3}\left(\nu_{1}-3 \nu_{2}\right)}-\frac{74.39 \beta_{2}^{2} \beta_{3}^{2} G^{4} M_{2} M_{3} P_{2} P_{3} \sqrt{-\frac{P_{1}\left(P_{1}-2 \Lambda_{1}\right)}{\Lambda_{1}}}}{\sqrt{\Lambda_{1}} \Lambda_{2}^{4} \Lambda_{3}^{3}\left(\nu_{1}-3 \nu_{2}\right)}$

$\begin{aligned} C_{4}^{1} & =\frac{7.41275 \beta_{2}^{2} \beta_{3}^{2} G^{4} M_{2} M_{3} P_{2}^{4} P_{3}^{2} \sqrt{-\frac{P_{1}\left(P_{1}-2 \Lambda_{1}\right)}{\Lambda_{1}}}-\frac{24.0914 \beta_{2}^{2} \beta_{3}^{2} G^{4} M_{2} M_{3} P_{2}^{3} P_{3}^{2} \sqrt{-\frac{P_{1}\left(P_{1}-2 \Lambda_{1}\right)}{\Lambda_{1}}}}{\sqrt{\Lambda_{1}} \Lambda_{2}^{6} \Lambda_{3}^{4}\left(\nu_{1}-3 \nu_{2}\right)}}{\sqrt{\Lambda_{1}} \Lambda_{2}^{7} \Lambda_{3}^{4}\left(\nu_{1}-3 \nu_{2}\right)}-\frac{18.53 \beta_{2}^{2} \beta_{3}^{2} G^{4} M_{2} M_{3} P_{2}^{2} P_{3}^{2} \sqrt{-\frac{P_{1}\left(P_{1}-2 \Lambda_{1}\right)}{\Lambda_{1}}}}{\sqrt{\Lambda_{1}} \Lambda_{2}^{5} \Lambda_{3}^{4}\left(\nu_{1}-3 \nu_{2}\right)} \\ + & \frac{8.88 \times 10^{-16} \beta_{2}^{2} \beta_{3}^{2} G^{4} M_{2} M_{3} P_{2} P_{3}^{2} \sqrt{-\frac{P_{1}\left(P_{1}-2 \Lambda_{1}\right)}{\Lambda_{1}}}}{\sqrt{\Lambda_{1}} \Lambda_{2}^{4} \Lambda_{3}^{4}\left(\nu_{1}-3 \nu_{2}\right)}+\frac{1}{}\end{aligned}$

$+\frac{48.1829 \beta_{2}^{2} \beta_{3}^{2} G^{4} M_{2} M_{3} P_{2}^{3} P_{3} \sqrt{-\frac{P_{1}\left(P_{1}-2 \Lambda_{1}\right)}{\Lambda_{1}}}}{\sqrt{\Lambda_{1}} \Lambda_{2}^{6} \Lambda_{3}^{3}\left(\nu_{1}-3 \nu_{2}\right)}-\frac{14.8255 \beta_{2}^{2} \beta_{3}^{2} G^{4} M_{2} M_{3} P_{2}^{4} P_{3} \sqrt{-\frac{P_{1}\left(P_{1}-2 \Lambda_{1}\right)}{\Lambda_{1}}}}{\sqrt{\Lambda_{1}} \Lambda_{2}^{7} \Lambda_{3}^{3}\left(\nu_{1}-3 \nu_{2}\right)}$

$-\frac{1.77 \times 10^{-15} \beta_{2}^{2} \beta_{3}^{2} G^{4} M_{2} M_{3} P_{2} P_{3} \sqrt{-\frac{P_{1}\left(P_{1}-2 \Lambda_{1}\right)}{\Lambda_{1}}}}{\sqrt{\Lambda_{1}} \Lambda_{2}^{4} \Lambda_{3}^{3}\left(\nu_{1}-3 \nu_{2}\right)}-\frac{37.06 \beta_{2}^{2} \beta_{3}^{2} G^{4} M_{2} M_{3} P_{2}^{2} P_{3} \sqrt{-\frac{P_{1}\left(P_{1}-2 \Lambda_{1}\right)}{\Lambda_{1}}}}{\sqrt{\Lambda_{1}} \Lambda_{2}^{5} \Lambda_{3}^{3}\left(\nu_{1}-3 \nu_{2}\right)}$ 


$$
\begin{aligned}
C_{4}^{2} & =\frac{2.00044 \beta_{2}^{2} \beta_{3}^{2} G^{4} M_{2} M_{3} P_{2}^{6} \sqrt{-\frac{P_{1}\left(P_{1}-2 \Lambda_{1}\right)}{\Lambda_{1}}}}{\sqrt{\Lambda_{1}} \Lambda_{2}^{9} \Lambda_{3}^{2}\left(\nu_{1}-3 \nu_{2}\right)}-\frac{9.43064 \beta_{2}^{2} \beta_{3}^{2} G^{4} M_{2} M_{3} P_{2}^{5} \sqrt{-\frac{P_{1}\left(P_{1}-2 \Lambda_{1}\right)}{\Lambda_{1}}}}{\sqrt{\Lambda_{1}} \Lambda_{2}^{8} \Lambda_{3}^{2}\left(\nu_{1}-3 \nu_{2}\right)} \\
& +\frac{15.3327 \beta_{2}^{2} \beta_{3}^{2} G^{4} M_{2} M_{3} P_{2}^{3} \sqrt{-\frac{P_{1}\left(P_{1}-2 \Lambda_{1}\right)}{\Lambda_{1}}}}{\sqrt{\Lambda_{1}} \Lambda_{2}^{6} \Lambda_{3}^{2}\left(\nu_{1}-3 \nu_{2}\right)}+\frac{7.43877 \beta_{2}^{2} \beta_{3}^{2} G^{4} M_{2} M_{3} P_{2}^{4} \sqrt{-\frac{P_{1}\left(P_{1}-2 \Lambda_{1}\right)}{\Lambda_{1}}}}{\sqrt{\Lambda_{1}} \Lambda_{2}^{7} \Lambda_{3}^{2}\left(\nu_{1}-3 \nu_{2}\right)}
\end{aligned}
$$

$-\frac{16.98 \beta_{2}^{2} \beta_{3}^{2} G^{4} M_{2} M_{3} P_{2}^{2} \sqrt{-\frac{P_{1}\left(P_{1}-2 \Lambda_{1}\right)}{\Lambda_{1}}}}{\sqrt{\Lambda_{1}} \Lambda_{2}^{5} \Lambda_{3}^{2}\left(\nu_{1}-3 \nu_{2}\right)}-\frac{7.412 \beta_{2}^{2} \beta_{3}^{2} G^{4} M_{2} M_{3} P_{2}^{4} P_{3}^{2} \sqrt{-\frac{P_{1}\left(P_{1}-2 \Lambda_{1}\right)}{\Lambda_{1}}}}{\sqrt{\Lambda_{1}} \Lambda_{2}^{7} \Lambda_{3}^{4}\left(\nu_{2}-2 \nu_{3}\right)}$

$+\frac{24.09 \beta_{2}^{2} \beta_{3}^{2} G^{4} M_{2} M_{3} P_{2}^{3} P_{3}^{2} \sqrt{-\frac{P_{1}\left(P_{1}-2 \Lambda_{1}\right)}{\Lambda_{1}}}}{\sqrt{\Lambda_{1}} \Lambda_{2}^{6} \Lambda_{3}^{4}\left(\nu_{2}-2 \nu_{3}\right)}-\frac{18.53 \beta_{2}^{2} \beta_{3}^{2} G^{4} M_{2} M_{3} P_{2}^{2} P_{3}^{2} \sqrt{-\frac{P_{1}\left(P_{1}-2 \Lambda_{1}\right)}{\Lambda_{1}}}}{\sqrt{\Lambda_{1}} \Lambda_{2}^{5} \Lambda_{3}^{4}\left(\nu_{2}-2 \nu_{3}\right)}$

$C_{5}^{1}=\frac{8.24579 \beta_{2}^{2} \beta_{3}^{2} G^{4} M_{2} M_{3} P_{1}^{2} P_{2}^{3} \sqrt{-\frac{P_{1}\left(P_{1}-2 \Lambda_{1}\right)}{\Lambda_{1}}} \sqrt{-\frac{P_{2}\left(P_{2}-2 \Lambda_{2}\right)}{\Lambda_{2}}} \sqrt{-\frac{P_{3}\left(P_{3}-2 \Lambda_{3}\right)}{\Lambda_{3}}}}{\Lambda_{1}^{5 / 2} \Lambda_{2}^{11 / 2} \Lambda_{3}^{5 / 2}\left(\nu_{1}-3 \nu_{2}\right)\left(P_{2}-2 \Lambda_{2}\right)}$

$-\frac{16.4916 \beta_{2}^{2} \beta_{3}^{2} G^{4} M_{2} M_{3} P_{1} P_{2}^{3} \sqrt{-\frac{P_{1}\left(P_{1}-2 \Lambda_{1}\right)}{\Lambda_{1}}} \sqrt{-\frac{P_{2}\left(P_{2}-2 \Lambda_{2}\right)}{\Lambda_{2}}} \sqrt{-\frac{P_{3}\left(P_{3}-2 \Lambda_{3}\right)}{\Lambda_{3}}}}{\Lambda_{1}^{3 / 2} \Lambda_{2}^{11 / 2} \Lambda_{3}^{5 / 2}\left(\nu_{1}-3 \nu_{2}\right)\left(P_{2}-2 \Lambda_{2}\right)}$

$\frac{30.2346 \beta_{2}^{2} \beta_{3}^{2} G^{4} M_{2} M_{3} P_{1}^{2} P_{2}^{2} \sqrt{-\frac{P_{1}\left(P_{1}-2 \Lambda_{1}\right)}{\Lambda_{1}}} \sqrt{-\frac{P_{2}\left(P_{2}-2 \Lambda_{2}\right)}{\Lambda_{2}}} \sqrt{-\frac{P_{3}\left(P_{3}-2 \Lambda_{3}\right)}{\Lambda_{3}}}}{\Lambda_{1}^{5 / 2} \Lambda_{2}^{9 / 2} \Lambda_{3}^{5 / 2}\left(\nu_{1}-3 \nu_{2}\right)\left(P_{2}-2 \Lambda_{2}\right)}$

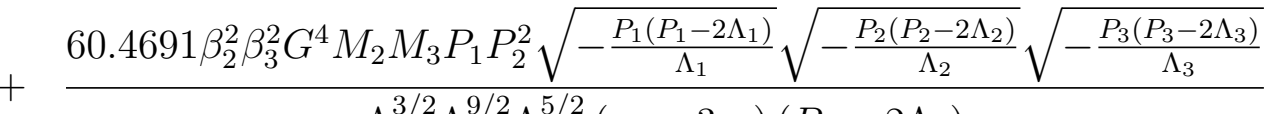
$\Lambda_{1}^{3 / 2} \Lambda_{2}^{9 / 2} \Lambda_{3}^{5 / 2}\left(\nu_{1}-3 \nu_{2}\right)\left(P_{2}-2 \Lambda_{2}\right)$

$\begin{aligned} C_{5}^{2} & =\frac{31.1508 \beta_{2}^{2} \beta_{3}^{2} G^{4} M_{2} M_{3} P_{1}^{2} P_{2} \sqrt{-\frac{P_{1}\left(P_{1}-2 \Lambda_{1}\right)}{\Lambda_{1}}} \sqrt{-\frac{P_{2}\left(P_{2}-2 \Lambda_{2}\right)}{\Lambda_{2}}} \sqrt{-\frac{P_{3}\left(P_{3}-2 \Lambda_{3}\right)}{\Lambda_{3}}}}{\Lambda_{1}^{5 / 2} \Lambda_{2}^{7 / 2} \Lambda_{3}^{5 / 2}\left(\nu_{1}-3 \nu_{2}\right)\left(P_{2}-2 \Lambda_{2}\right)} \\ - & \frac{62.3015 \beta_{2}^{2} \beta_{3}^{2} G^{4} M_{2} M_{3} P_{1} P_{2} \sqrt{-\frac{P_{1}\left(P_{1}-2 \Lambda_{1}\right)}{\Lambda_{1}}} \sqrt{-\frac{P_{2}\left(P_{2}-2 \Lambda_{2}\right)}{\Lambda_{2}}} \sqrt{-\frac{P_{3}\left(P_{3}-2 \Lambda_{3}\right)}{\Lambda_{3}}}}{\Lambda_{1}^{3 / 2} \Lambda_{2}^{7 / 2} \Lambda_{3}^{5 / 2}\left(\nu_{1}-3 \nu_{2}\right)\left(P_{2}-2 \Lambda_{2}\right)}\end{aligned}$

$-\frac{7.32959 \beta_{2}^{2} \beta_{3}^{2} G^{4} M_{2} M_{3} P_{1}^{2} \sqrt{-\frac{P_{1}\left(P_{1}-2 \Lambda_{1}\right)}{\Lambda_{1}}} \sqrt{-\frac{P_{2}\left(P_{2}-2 \Lambda_{2}\right)}{\Lambda_{2}}} \sqrt{-\frac{P_{3}\left(P_{3}-2 \Lambda_{3}\right)}{\Lambda_{3}}}}{\Lambda_{1}^{5 / 2} \Lambda_{2}^{5 / 2} \Lambda_{3}^{5 / 2}\left(\nu_{1}-3 \nu_{2}\right)\left(P_{2}-2 \Lambda_{2}\right)}$

$+\frac{14.6592 \beta_{2}^{2} \beta_{3}^{2} G^{4} M_{2} M_{3} P_{1} \sqrt{-\frac{P_{1}\left(P_{1}-2 \Lambda_{1}\right)}{\Lambda_{1}}} \sqrt{-\frac{P_{2}\left(P_{2}-2 \Lambda_{2}\right)}{\Lambda_{2}}} \sqrt{-\frac{P_{3}\left(P_{3}-2 \Lambda_{3}\right)}{\Lambda_{3}}}}{\Lambda_{1}^{3 / 2} \Lambda_{2}^{5 / 2} \Lambda_{3}^{5 / 2}\left(\nu_{1}-3 \nu_{2}\right)\left(P_{2}-2 \Lambda_{2}\right)}$ 


$$
\begin{aligned}
C_{6}^{1} & =\frac{24.0651 \beta_{2}^{2} \beta_{3}^{2} G^{4} M_{2} M_{3} P_{2}^{6} \sqrt{-\frac{P_{3}\left(P_{3}-2 \Lambda_{3}\right)}{\Lambda_{3}}}}{\Lambda_{2}^{9} \Lambda_{3}^{5 / 2}\left(\nu_{1}-3 \nu_{2}\right)}-\frac{140.38 \beta_{2}^{2} \beta_{3}^{2} G^{4} M_{2} M_{3} P_{2}^{5} \sqrt{-\frac{P_{3}\left(P_{3}-2 \Lambda_{3}\right)}{\Lambda_{3}}}}{\Lambda_{2}^{8} \Lambda_{3}^{5 / 2}\left(\nu_{1}-3 \nu_{2}\right)} \\
& +\frac{318.56 \beta_{2}^{2} \beta_{3}^{2} G^{4} M_{2} M_{3} P_{2}^{4} \sqrt{-\frac{P_{3}\left(P_{3}-2 \Lambda_{3}\right)}{\Lambda_{3}}}}{\Lambda_{2}^{7} \Lambda_{3}^{5 / 2}\left(\nu_{1}-3 \nu_{2}\right)}+\frac{40.105 \beta_{2}^{2} \beta_{3}^{2} G^{4} M_{2} M_{3} P_{1}^{2} P_{2}^{4} \sqrt{-\frac{P_{3}\left(P_{3}-2 \Lambda_{3}\right)}{\Lambda_{3}}}}{\Lambda_{1}^{2} \Lambda_{2}^{7} \Lambda_{3}^{5 / 2}\left(\nu_{1}-3 \nu_{2}\right)}
\end{aligned}
$$

$$
\begin{aligned}
& -\frac{347.855 \beta_{2}^{2} \beta_{3}^{2} G^{4} M_{2} M_{3} P_{2}^{3} \sqrt{-\frac{P_{3}\left(P_{3}-2 \Lambda_{3}\right)}{\Lambda_{3}}}}{\Lambda_{2}^{6} \Lambda_{3}^{5 / 2}\left(\nu_{1}-3 \nu_{2}\right)}-\frac{80.21 \beta_{2}^{2} \beta_{3}^{2} G^{4} M_{2} M_{3} P_{1} P_{2}^{4} \sqrt{-\frac{P_{3}\left(P_{3}-2 \Lambda_{3}\right)}{\Lambda_{3}}}}{\Lambda_{1} \Lambda_{2}^{7} \Lambda_{3}^{5 / 2}\left(\nu_{1}-3 \nu_{2}\right)} \\
& +\frac{288.76 \beta_{2}^{2} \beta_{3}^{2} G^{4} M_{2} M_{3} P_{1} P_{2}^{3} \sqrt{-\frac{P_{3}\left(P_{3}-2 \Lambda_{3}\right)}{\Lambda_{3}}}}{\Lambda_{1} \Lambda_{2}^{6} \Lambda_{3}^{5 / 2}\left(\nu_{1}-3 \nu_{2}\right)}-\frac{144.38 \beta_{2}^{2} \beta_{3}^{2} G^{4} M_{2} M_{3} P_{1}^{2} P_{2}^{3} \sqrt{-\frac{P_{3}\left(P_{3}-2 \Lambda_{3}\right)}{\Lambda_{3}}}}{\Lambda_{1}^{2} \Lambda_{2}^{6} \Lambda_{3}^{5 / 2}\left(\nu_{1}-3 \nu_{2}\right)}
\end{aligned}
$$

$$
\begin{aligned}
C_{6}^{2} & =\frac{171.38 \beta_{2}^{2} \beta_{3}^{2} G^{4} M_{2} M_{3} P_{2}^{2} \sqrt{-\frac{P_{3}\left(P_{3}-2 \Lambda_{3}\right)}{\Lambda_{3}}}}{\Lambda_{2}^{5} \Lambda_{3}^{5 / 2}\left(\nu_{1}-3 \nu_{2}\right)}-\frac{5.85 \beta_{2}^{2} \beta_{3}^{2} G^{4} M_{2} M_{3} P_{1}^{4} P_{2}^{2} \sqrt{-\frac{P_{3}\left(P_{3}-2 \Lambda_{3}\right)}{\Lambda_{3}}}}{\Lambda_{1}^{4} \Lambda_{2}^{5} \Lambda_{3}^{5 / 2}\left(\nu_{1}-3 \nu_{2}\right)} \\
& +\frac{127.43 \beta_{2}^{2} \beta_{3}^{2} G^{4} M_{2} M_{3} P_{1}^{2} P_{2}^{2} \sqrt{-\frac{P_{3}\left(P_{3}-2 \Lambda_{3}\right)}{\Lambda_{3}}}}{\Lambda_{1}^{2} \Lambda_{2}^{5} \Lambda_{3}^{5 / 2}\left(\nu_{1}-3 \nu_{2}\right)}+\frac{23.4 \beta_{2}^{2} \beta_{3}^{2} G^{4} M_{2} M_{3} P_{1}^{3} P_{2}^{2} \sqrt{-\frac{P_{3}\left(P_{3}-2 \Lambda_{3}\right)}{\Lambda_{3}}}}{\Lambda_{1}^{3} \Lambda_{2}^{5} \Lambda_{3}^{5 / 2}\left(\nu_{1}-3 \nu_{2}\right)}
\end{aligned}
$$

$$
\begin{aligned}
& -\frac{23.82 \beta_{2}^{2} \beta_{3}^{2} G^{4} M_{2} M_{3} P_{2} \sqrt{-\frac{P_{3}\left(P_{3}-2 \Lambda_{3}\right)}{\Lambda_{3}}}}{\Lambda_{2}^{4} \Lambda_{3}^{5 / 2}\left(\nu_{1}-3 \nu_{2}\right)}-\frac{301.67 \beta_{2}^{2} \beta_{3}^{2} G^{4} M_{2} M_{3} P_{1} P_{2}^{2} \sqrt{-\frac{P_{3}\left(P_{3}-2 \Lambda_{3}\right)}{\Lambda_{3}}}}{\Lambda_{1} \Lambda_{2}^{5} \Lambda_{3}^{5 / 2}\left(\nu_{1}-3 \nu_{2}\right)} \\
& +\frac{7.32 \beta_{2}^{2} \beta_{3}^{2} G^{4} M_{2} M_{3} P_{1}^{4} P_{2} \sqrt{-\frac{P_{3}\left(P_{3}-2 \Lambda_{3}\right)}{\Lambda_{3}}}}{\Lambda_{1}^{4} \Lambda_{2}^{4} \Lambda_{3}^{5 / 2}\left(\nu_{1}-3 \nu_{2}\right)}-\frac{29.2561 \beta_{2}^{2} \beta_{3}^{2} G^{4} M_{2} M_{3} P_{1}^{3} P_{2} \sqrt{-\frac{P_{3}\left(P_{3}-2 \Lambda_{3}\right)}{\Lambda_{3}}}}{\Lambda_{1}^{3} \Lambda_{2}^{4} \Lambda_{3}^{5 / 2}\left(\nu_{1}-3 \nu_{2}\right)}
\end{aligned}
$$

$$
\begin{aligned}
& C_{7}^{1}=\frac{59.0723 \beta_{2}^{2} \beta_{3}^{2} G^{4} M_{2} M_{3} P_{2}^{5} \sqrt{-\frac{P_{1}\left(P_{1}-2 \Lambda_{1}\right)}{\Lambda_{1}}} \sqrt{-\frac{P_{2}\left(P_{2}-2 \Lambda_{2}\right)}{\Lambda_{2}}} \sqrt{-\frac{P_{3}\left(P_{3}-2 \Lambda_{3}\right)}{\Lambda_{3}}}}{\sqrt{\Lambda_{1}} \Lambda_{2}^{15 / 2} \Lambda_{3}^{5 / 2}\left(\nu_{1}-3 \nu_{2}\right)\left(P_{2}-2 \Lambda_{2}\right)} \\
& 327.583 \beta_{2}^{2} \beta_{3}^{2} G^{4} M_{2} M_{3} P_{2}^{4} \sqrt{-\frac{P_{1}\left(P_{1}-2 \Lambda_{1}\right)}{\Lambda_{1}}} \sqrt{-\frac{P_{2}\left(P_{2}-2 \Lambda_{2}\right)}{\Lambda_{2}}} \sqrt{-\frac{P_{3}\left(P_{3}-2 \Lambda_{3}\right)}{\Lambda_{3}}} \\
& \sqrt{\Lambda_{1}} \Lambda_{2}^{13 / 2} \Lambda_{3}^{5 / 2}\left(\nu_{1}-3 \nu_{2}\right)\left(P_{2}-2 \Lambda_{2}\right) \\
& +\frac{6.45593 \beta_{2}^{2} \beta_{3}^{2} G^{4} M_{2} M_{3} P_{1}^{2} P_{2}^{3} \sqrt{-\frac{P_{1}\left(P_{1}-2 \Lambda_{1}\right)}{\Lambda_{1}}} \sqrt{-\frac{P_{2}\left(P_{2}-2 \Lambda_{2}\right)}{\Lambda_{2}}} \sqrt{-\frac{P_{3}\left(P_{3}-2 \Lambda_{3}\right)}{\Lambda_{3}}}}{\Lambda_{1}^{5 / 2} \Lambda_{2}^{11 / 2} \Lambda_{3}^{5 / 2}\left(\nu_{1}-3 \nu_{2}\right)\left(P_{2}-2 \Lambda_{2}\right)} \\
& -\frac{12.9119 \beta_{2}^{2} \beta_{3}^{2} G^{4} M_{2} M_{3} P_{1} P_{2}^{3} \sqrt{-\frac{P_{1}\left(P_{1}-2 \Lambda_{1}\right)}{\Lambda_{1}}} \sqrt{-\frac{P_{2}\left(P_{2}-2 \Lambda_{2}\right)}{\Lambda_{2}}} \sqrt{-\frac{P_{3}\left(P_{3}-2 \Lambda_{3}\right)}{\Lambda_{3}}}}{\Lambda_{1}^{3 / 2} \Lambda_{2}^{11 / 2} \Lambda_{3}^{5 / 2}\left(\nu_{1}-3 \nu_{2}\right)\left(P_{2}-2 \Lambda_{2}\right)}
\end{aligned}
$$




$$
\begin{aligned}
C_{7}^{2} & =\frac{651.259 \beta_{2}^{2} \beta_{3}^{2} G^{4} M_{2} M_{3} P_{2}^{3} \sqrt{-\frac{P_{1}\left(P_{1}-2 \Lambda_{1}\right)}{\Lambda_{1}}} \sqrt{-\frac{P_{2}\left(P_{2}-2 \Lambda_{2}\right)}{\Lambda_{2}}} \sqrt{-\frac{P_{3}\left(P_{3}-2 \Lambda_{3}\right)}{\Lambda_{3}}}}{\sqrt{\Lambda_{1}} \Lambda_{2}^{11 / 2} \Lambda_{3}^{5 / 2}\left(\nu_{1}-3 \nu_{2}\right)\left(P_{2}-2 \Lambda_{2}\right)} \\
& -\frac{20.8024 \beta_{2}^{2} \beta_{3}^{2} G^{4} M_{2} M_{3} P_{1}^{2} P_{2}^{2} \sqrt{-\frac{P_{1}\left(P_{1}-2 \Lambda_{1}\right)}{\Lambda_{1}}} \sqrt{-\frac{P_{2}\left(P_{2}-2 \Lambda_{2}\right)}{\Lambda_{2}}} \sqrt{-\frac{P_{3}\left(P_{3}-2 \Lambda_{3}\right)}{\Lambda_{3}}}}{\Lambda_{1}^{5 / 2} \Lambda_{2}^{9 / 2} \Lambda_{3}^{5 / 2}\left(\nu_{1}-3 \nu_{2}\right)\left(P_{2}-2 \Lambda_{2}\right)} \\
+ & \frac{41.6049 \beta_{2}^{2} \beta_{3}^{2} G^{4} M_{2} M_{3} P_{1} P_{2}^{2} \sqrt{-\frac{P_{1}\left(P_{1}-2 \Lambda_{1}\right)}{\Lambda_{1}}} \sqrt{-\frac{P_{2}\left(P_{2}-2 \Lambda_{2}\right)}{\Lambda_{2}}} \sqrt{-\frac{P_{3}\left(P_{3}-2 \Lambda_{3}\right)}{\Lambda_{3}}}}{\Lambda_{1}^{3 / 2} \Lambda_{2}^{9 / 2} \Lambda_{3}^{5 / 2}\left(\nu_{1}-3 \nu_{2}\right)\left(P_{2}-2 \Lambda_{2}\right)} \\
- & \frac{542.335 \beta_{2}^{2} \beta_{3}^{2} G^{4} M_{2} M_{3} P_{2}^{2} \sqrt{-\frac{P_{1}\left(P_{1}-2 \Lambda_{1}\right)}{\Lambda_{1}}} \sqrt{-\frac{P_{2}\left(P_{2}-2 \Lambda_{2}\right)}{\Lambda_{2}}} \sqrt{-\frac{P_{3}\left(P_{3}-2 \Lambda_{3}\right)}{\Lambda_{3}}}}{\sqrt{\Lambda_{1}} \Lambda_{2}^{9 / 2} \Lambda_{3}^{5 / 2}\left(\nu_{1}-3 \nu_{2}\right)\left(P_{2}-2 \Lambda_{2}\right)}
\end{aligned}
$$

$$
\begin{aligned}
C_{8}^{1} & =-\frac{12.7829 \beta_{2}^{2} \beta_{3}^{2} G^{4} M_{2} M_{3} P_{2}^{5} \sqrt{-\frac{P_{1}\left(P_{1}-2 \Lambda_{1}\right)}{\Lambda_{1}}} \sqrt{-\frac{P_{2}\left(P_{2}-2 \Lambda_{2}\right)}{\Lambda_{2}}} \sqrt{-\frac{P_{3}\left(P_{3}-2 \Lambda_{3}\right)}{\Lambda_{3}}}}{\sqrt{\Lambda_{1}} \Lambda_{2}^{15 / 2} \Lambda_{3}^{5 / 2}\left(\nu_{1}-3 . \nu_{2}\right)\left(P_{2}-2 . \Lambda_{2}\right)} \\
& +\frac{66.2384 \beta_{2}^{2} \beta_{3}^{2} G^{4} M_{2} M_{3} P_{2}^{4} \sqrt{-\frac{P_{1}\left(P_{1}-2 \Lambda_{1}\right)}{\Lambda_{1}}} \sqrt{-\frac{P_{2}\left(P_{2}-2 \Lambda_{2}\right)}{\Lambda_{2}}} \sqrt{-\frac{P_{3}\left(P_{3}-2 \Lambda_{3}\right)}{\Lambda_{3}}}}{\sqrt{\Lambda_{1}} \Lambda_{2}^{13 / 2} \Lambda_{3}^{5 / 2}\left(\nu_{1}-3 . \nu_{2}\right)\left(P_{2}-2 . \Lambda_{2}\right)} \\
& -\frac{111.559 \beta_{2}^{2} \beta_{3}^{2} G^{4} M_{2} M_{3} P_{2}^{3} \sqrt{-\frac{P_{1}\left(P_{1}-2 \Lambda_{1}\right)}{\Lambda_{1}}} \sqrt{-\frac{P_{2}\left(P_{2}-2 \Lambda_{2}\right)}{\Lambda_{2}}} \sqrt{-\frac{P_{3}\left(P_{3}-2 \Lambda_{3}\right)}{\Lambda_{3}}}}{\sqrt{\Lambda_{1}} \Lambda_{2}^{11 / 2} \Lambda_{3}^{5 / 2}\left(\nu_{1}-3 . \nu_{2}\right)\left(P_{2}-2 . \Lambda_{2}\right)} \\
+ & \frac{60.4281 \beta_{2}^{2} \beta_{3}^{2} G^{4} M_{2} M_{3} P_{2}^{2} \sqrt{-\frac{P_{1}\left(P_{1}-2 \Lambda_{1}\right)}{\Lambda_{1}}} \sqrt{-\frac{P_{2}\left(P_{2}-2 \Lambda_{2}\right)}{\Lambda_{2}}} \sqrt{-\frac{P_{3}\left(P_{3}-2 \Lambda_{3}\right)}{\Lambda_{3}}}}{\sqrt{\Lambda_{1}} \Lambda_{2}^{9 / 2} \Lambda_{3}^{5 / 2}\left(\nu_{1}-3 . \nu_{2}\right)\left(P_{2}-2 . \Lambda_{2}\right)}
\end{aligned}
$$$$
C_{8}^{2}=-\frac{3.55 \times 10^{-15} \beta_{2}^{2} \beta_{3}^{2} G^{4} M_{2} M_{3} P_{2} \sqrt{-\frac{P_{1}\left(P_{1}-2 \Lambda_{1}\right)}{\Lambda_{1}}} \sqrt{-\frac{P_{2}\left(P_{2}-2 \Lambda_{2}\right)}{\Lambda_{2}}} \sqrt{-\frac{P_{3}\left(P_{3}-2 \Lambda_{3}\right)}{\Lambda_{3}}}}{\sqrt{\Lambda_{1}} \Lambda_{2}^{7 / 2} \Lambda_{3}^{5 / 2}\left(\nu_{1}-3 . \nu_{2}\right)\left(P_{2}-2 . \Lambda_{2}\right)}
$$$$
+\frac{12.7829 \beta_{2}^{2} \beta_{3}^{2} G^{4} M_{2} M_{3} P_{2}^{4} \sqrt{-\frac{P_{1}\left(P_{1}-2 \Lambda_{1}\right)}{\Lambda_{1}}} \sqrt{-\frac{P_{2}\left(P_{2}-2 \Lambda_{2}\right)}{\Lambda_{2}}} \sqrt{-\frac{P_{3}\left(P_{3}-2 \Lambda_{3}\right)}{\Lambda_{3}}}}{\sqrt{\Lambda_{1}} \Lambda_{2}^{15 / 2} \Lambda_{3}^{5 / 2}\left(\nu_{2}-2 . \nu_{3}\right)}
$$$$
-\frac{40.6727 \beta_{2}^{2} \beta_{3}^{2} G^{4} M_{2} M_{3} P_{2}^{3} \sqrt{-\frac{P_{1}\left(P_{1}-2 \Lambda_{1}\right)}{\Lambda_{1}}} \sqrt{-\frac{P_{2}\left(P_{2}-2 \Lambda_{2}\right)}{\Lambda_{2}}} \sqrt{-\frac{P_{3}\left(P_{3}-2 \Lambda_{3}\right)}{\Lambda_{3}}}}{\sqrt{\Lambda_{1}} \Lambda_{2}^{13 / 2} \Lambda_{3}^{5 / 2}\left(\nu_{2}-2 . \nu_{3}\right)}
$$$$
+\frac{30.214 \beta_{2}^{2} \beta_{3}^{2} G^{4} M_{2} M_{3} P_{2}^{2} \sqrt{-\frac{P_{1}\left(P_{1}-2 \Lambda_{1}\right)}{\Lambda_{1}}} \sqrt{-\frac{P_{2}\left(P_{2}-2 \Lambda_{2}\right)}{\Lambda_{2}}} \sqrt{-\frac{P_{3}\left(P_{3}-2 \Lambda_{3}\right)}{\Lambda_{3}}}}{\sqrt{\Lambda_{1}} \Lambda_{2}^{11 / 2} \Lambda_{3}^{5 / 2}\left(\nu_{2}-2 . \nu_{3}\right)}
$$ 


$$
\begin{aligned}
C_{9}^{1} & =\frac{18.90 \beta_{2}^{2} \beta_{3}^{2} G^{4} M_{2} M_{3} P_{2}^{3} P_{3}^{2} \sqrt{-\frac{P_{3}\left(P_{3}-2 \Lambda_{3}\right)}{\Lambda_{3}}}}{\Lambda_{2}^{6} \Lambda_{3}^{9 / 2}\left(\nu_{1}-3 \nu_{2}\right)}-\frac{5.67 \beta_{2}^{2} \beta_{3}^{2} G^{4} M_{2} M_{3} P_{2}^{4} P_{3}^{2} \sqrt{-\frac{P_{3}\left(P_{3}-2 \Lambda_{3}\right)}{\Lambda_{3}}}}{\Lambda_{2}^{7} \Lambda_{3}^{9 / 2}\left(\nu_{1}-3 \nu_{2}\right)} \\
& -\frac{20.74 \beta_{2}^{2} \beta_{3}^{2} G^{4} M_{2} M_{3} P_{2}^{2} P_{3}^{2} \sqrt{-\frac{P_{3}\left(P_{3}-2 \Lambda_{3}\right)}{\Lambda_{3}}}}{\Lambda_{2}^{5} \Lambda_{3}^{9 / 2}\left(\nu_{1}-3 \nu_{2}\right)}-\frac{7.56 \beta_{2}^{2} \beta_{3}^{2} G^{4} M_{2} M_{3} P_{1}^{2} P_{2}^{2} P_{3}^{2} \sqrt{-\frac{P_{3}\left(P_{3}-2 \Lambda_{3}\right)}{\Lambda_{3}}}}{\Lambda_{1}^{2} \Lambda_{2}^{5} \Lambda_{3}^{9 / 2}\left(\nu_{1}-3 \nu_{2}\right)}
\end{aligned}
$$

$+\frac{8.42 \beta_{2}^{2} \beta_{3}^{2} G^{4} M_{2} M_{3} P_{2} P_{3}^{2} \sqrt{-\frac{P_{3}\left(P_{3}-2 \Lambda_{3}\right)}{\Lambda_{3}}}}{\Lambda_{2}^{4} \Lambda_{3}^{9 / 2}\left(\nu_{1}-3 \nu_{2}\right)}+\frac{15.1202 \beta_{2}^{2} \beta_{3}^{2} G^{4} M_{2} M_{3} P_{1} P_{2}^{2} P_{3}^{2} \sqrt{-\frac{P_{3}\left(P_{3}-2 \Lambda_{3}\right)}{\Lambda_{3}}}}{\Lambda_{1} \Lambda_{2}^{5} \Lambda_{3}^{9 / 2}\left(\nu_{1}-3 \nu_{2}\right)}$

$+\frac{11.34 \beta_{2}^{2} \beta_{3}^{2} G^{4} M_{2} M_{3} P_{1}^{2} P_{2} P_{3}^{2} \sqrt{-\frac{P_{3}\left(P_{3}-2 \Lambda_{3}\right)}{\Lambda_{3}}}}{\Lambda_{1}^{2} \Lambda_{2}^{4} \Lambda_{3}^{9 / 2}\left(\nu_{1}-3 \nu_{2}\right)}-\frac{22.68 \beta_{2}^{2} \beta_{3}^{2} G^{4} M_{2} M_{3} P_{1} P_{2} P_{3}^{2} \sqrt{-\frac{P_{3}\left(P_{3}-2 \Lambda_{3}\right)}{\Lambda_{3}}}}{\Lambda_{1} \Lambda_{2}^{4} \Lambda_{3}^{9 / 2}\left(\nu_{1}-3 \nu_{2}\right)}$

$\begin{aligned} C_{9}^{2} & =\frac{0.69 \beta_{2}^{2} \beta_{3}^{2} G^{4} M_{2} M_{3} P_{1}^{4} P_{3}^{2} \sqrt{-\frac{P_{3}\left(P_{3}-2 \Lambda_{3}\right)}{\Lambda_{3}}}}{\Lambda_{1}^{4} \Lambda_{2}^{3} \Lambda_{3}^{9 / 2}\left(\nu_{1}-3 \nu_{2}\right)}-\frac{2.7575 \beta_{2}^{2} \beta_{3}^{2} G^{4} M_{2} M_{3} P_{1}^{3} P_{3}^{2} \sqrt{-\frac{P_{3}\left(P_{3}-2 \Lambda_{3}\right)}{\Lambda_{3}}}}{\Lambda_{1}^{3} \Lambda_{2}^{3} \Lambda_{3}^{9 / 2}\left(\nu_{1}-3 \nu_{2}\right)} \\ & +\frac{1.52 \beta_{2}^{2} \beta_{3}^{2} G^{4} M_{2} M_{3} P_{1} P_{3}^{2} \sqrt{-\frac{P_{3}\left(P_{3}-2 \Lambda_{3}\right)}{\Lambda_{3}}}}{\Lambda_{1} \Lambda_{2}^{3} \Lambda_{3}^{9 / 2}\left(\nu_{1}-3 \nu_{2}\right)}+\frac{1.99702 \beta_{2}^{2} \beta_{3}^{2} G^{4} M_{2} M_{3} P_{1}^{2} P_{3}^{2} \sqrt{-\frac{P_{3}\left(P_{3}-2 \Lambda_{3}\right)}{\Lambda_{3}}}}{\Lambda_{1}^{2} \Lambda_{2}^{3} \Lambda_{3}^{9 / 2}\left(\nu_{1}-3 \nu_{2}\right)}\end{aligned}$

$+\frac{11.3411 \beta_{2}^{2} \beta_{3}^{2} G^{4} M_{2} M_{3} P_{2}^{4} P_{3} \sqrt{-\frac{P_{3}\left(P_{3}-2 \Lambda_{3}\right)}{\Lambda_{3}}}}{\Lambda_{2}^{7} \Lambda_{3}^{7 / 2}\left(\nu_{1}-3 \nu_{2}\right)}-\frac{37.80 \beta_{2}^{2} \beta_{3}^{2} G^{4} M_{2} M_{3} P_{2}^{3} P_{3} \sqrt{-\frac{P_{3}\left(P_{3}-2 \Lambda_{3}\right)}{\Lambda_{3}}}}{\Lambda_{2}^{6} \Lambda_{3}^{7 / 2}\left(\nu_{1}-3 \nu_{2}\right)}$

$+\frac{41.47 \beta_{2}^{2} \beta_{3}^{2} G^{4} M_{2} M_{3} P_{2}^{2} P_{3} \sqrt{-\frac{P_{3}\left(P_{3}-2 \Lambda_{3}\right)}{\Lambda_{3}}}}{\Lambda_{2}^{5} \Lambda_{3}^{7 / 2}\left(\nu_{1}-3 \nu_{2}\right)}+\frac{15.12 \beta_{2}^{2} \beta_{3}^{2} G^{4} M_{2} M_{3} P_{1}^{2} P_{2}^{2} P_{3} \sqrt{-\frac{P_{3}\left(P_{3}-2 \Lambda_{3}\right)}{\Lambda_{3}}}}{\Lambda_{1}^{2} \Lambda_{2}^{5} \Lambda_{3}^{7 / 2}\left(\nu_{1}-3 \nu_{2}\right)}$

$C_{10}^{1}=-\frac{1.295 \beta_{2}^{2} \beta_{3}^{2} G^{4} M_{2} M_{3} P_{1}^{2} P_{2} P_{3}^{2} \sqrt{-\frac{P_{1}\left(P_{1}-2 \Lambda_{1}\right)}{\Lambda_{1}}} \sqrt{-\frac{P_{2}\left(P_{2}-2 \Lambda_{2}\right)}{\Lambda_{2}}} \sqrt{-\frac{P_{3}\left(P_{3}-2 \Lambda_{3}\right)}{\Lambda_{3}}}}{\Lambda_{1}^{5 / 2} \Lambda_{2}^{7 / 2} \Lambda_{3}^{9 / 2}\left(\nu_{1}-3 \nu_{2}\right)\left(P_{2}-2 \Lambda_{2}\right)}$

$+\frac{2.59 \beta_{2}^{2} \beta_{3}^{2} G^{4} M_{2} M_{3} P_{1} P_{2} P_{3}^{2} \sqrt{-\frac{P_{1}\left(P_{1}-2 \Lambda_{1}\right)}{\Lambda_{1}}} \sqrt{-\frac{P_{2}\left(P_{2}-2 \Lambda_{2}\right)}{\Lambda_{2}}} \sqrt{-\frac{P_{3}\left(P_{3}-2 \Lambda_{3}\right)}{\Lambda_{3}}}}{\Lambda_{1}^{3 / 2} \Lambda_{2}^{7 / 2} \Lambda_{3}^{9 / 2}\left(\nu_{1}-3 \nu_{2}\right)\left(P_{2}-2 \Lambda_{2}\right)}$

$+\frac{2.158 \beta_{2}^{2} \beta_{3}^{2} G^{4} M_{2} M_{3} P_{1}^{2} P_{3}^{2} \sqrt{-\frac{P_{1}\left(P_{1}-2 \Lambda_{1}\right)}{\Lambda_{1}}} \sqrt{-\frac{P_{2}\left(P_{2}-2 \Lambda_{2}\right)}{\Lambda_{2}}} \sqrt{-\frac{P_{3}\left(P_{3}-2 \Lambda_{3}\right)}{\Lambda_{3}}}}{\Lambda_{1}^{5 / 2} \Lambda_{2}^{5 / 2} \Lambda_{3}^{9 / 2}\left(\nu_{1}-3 \nu_{2}\right)\left(P_{2}-2 \Lambda_{2}\right)}$

$-\frac{4.317 \beta_{2}^{2} \beta_{3}^{2} G^{4} M_{2} M_{3} P_{1} P_{3}^{2} \sqrt{-\frac{P_{1}\left(P_{1}-2 \Lambda_{1}\right)}{\Lambda_{1}}} \sqrt{-\frac{P_{2}\left(P_{2}-2 \Lambda_{2}\right)}{\Lambda_{2}}} \sqrt{-\frac{P_{3}\left(P_{3}-2 \Lambda_{3}\right)}{\Lambda_{3}}}}{\Lambda_{1}^{3 / 2} \Lambda_{2}^{5 / 2} \Lambda_{3}^{9 / 2}\left(\nu_{1}-3 \nu_{2}\right)\left(P_{2}-2 \Lambda_{2}\right)}$ 


$$
\begin{aligned}
C_{10}^{2} & =\frac{2.59 \beta_{2}^{2} \beta_{3}^{2} G^{4} M_{2} M_{3} P_{1}^{2} P_{2} P_{3} \sqrt{-\frac{P_{1}\left(P_{1}-2 \Lambda_{1}\right)}{\Lambda_{1}}} \sqrt{-\frac{P_{2}\left(P_{2}-2 \Lambda_{2}\right)}{\Lambda_{2}}} \sqrt{-\frac{P_{3}\left(P_{3}-2 \Lambda_{3}\right)}{\Lambda_{3}}}}{\Lambda_{1}^{5 / 2} \Lambda_{2}^{7 / 2} \Lambda_{3}^{7 / 2}\left(\nu_{1}-3 \nu_{2}\right)\left(P_{2}-2 \Lambda_{2}\right)} \\
- & \frac{5.18 \beta_{2}^{2} \beta_{3}^{2} G^{4} M_{2} M_{3} P_{1} P_{2} P_{3} \sqrt{-\frac{P_{1}\left(P_{1}-2 \Lambda_{1}\right)}{\Lambda_{1}}} \sqrt{-\frac{P_{2}\left(P_{2}-2 \Lambda_{2}\right)}{\Lambda_{2}}} \sqrt{-\frac{P_{3}\left(P_{3}-2 \Lambda_{3}\right)}{\Lambda_{3}}}}{\Lambda_{1}^{3 / 2} \Lambda_{2}^{7 / 2} \Lambda_{3}^{7 / 2}\left(\nu_{1}-3 \nu_{2}\right)\left(P_{2}-2 \Lambda_{2}\right)} \\
- & \frac{4.31776 \beta_{2}^{2} \beta_{3}^{2} G^{4} M_{2} M_{3} P_{1}^{2} P_{3} \sqrt{-\frac{P_{1}\left(P_{1}-2 \Lambda_{1}\right)}{\Lambda_{1}}} \sqrt{-\frac{P_{2}\left(P_{2}-2 \Lambda_{2}\right)}{\Lambda_{2}}} \sqrt{-\frac{P_{3}\left(P_{3}-2 \Lambda_{3}\right)}{\Lambda_{3}}}}{\Lambda_{1}^{5 / 2} \Lambda_{2}^{5 / 2} \Lambda_{3}^{7 / 2}\left(\nu_{1}-3 \nu_{2}\right)\left(P_{2}-2 \Lambda_{2}\right)} \\
+ & \frac{8.63 \beta_{2}^{2} \beta_{3}^{2} G^{4} M_{2} M_{3} P_{1} P_{3} \sqrt{-\frac{P_{1}\left(P_{1}-2 \Lambda_{1}\right)}{\Lambda_{1}}} \sqrt{-\frac{P_{2}\left(P_{2}-2 \Lambda_{2}\right)}{\Lambda_{2}}} \sqrt{-\frac{P_{3}\left(P_{3}-2 \Lambda_{3}\right)}{\Lambda_{3}}}}{\Lambda_{1}^{3 / 2} \Lambda_{2}^{5 / 2} \Lambda_{3}^{7 / 2}\left(\nu_{1}-3 \nu_{2}\right)\left(P_{2}-2 \Lambda_{2}\right)}
\end{aligned}
$$$$
C_{11}^{1}=-\frac{12.65 \beta_{2}^{2} \beta_{3}^{2} G^{4} M_{2} M_{3} P_{2}^{3} P_{3}^{2} \sqrt{-\frac{P_{1}\left(P_{1}-2 \Lambda_{1}\right)}{\Lambda_{1}}} \sqrt{-\frac{P_{2}\left(P_{2}-2 \Lambda_{2}\right)}{\Lambda_{2}}} \sqrt{-\frac{P_{3}\left(P_{3}-2 \Lambda_{3}\right)}{\Lambda_{3}}}}{\sqrt{\Lambda_{1}} \Lambda_{2}^{11 / 2} \Lambda_{3}^{9 / 2}\left(\nu_{1}-3 \nu_{2}\right)\left(P_{2}-2 \Lambda_{2}\right)}
$$$$
+\frac{43.02 \beta_{2}^{2} \beta_{3}^{2} G^{4} M_{2} M_{3} P_{2}^{2} P_{3}^{2} \sqrt{-\frac{P_{1}\left(P_{1}-2 \Lambda_{1}\right)}{\Lambda_{1}}} \sqrt{-\frac{P_{2}\left(P_{2}-2 \Lambda_{2}\right)}{\Lambda_{2}}} \sqrt{-\frac{P_{3}\left(P_{3}-2 \Lambda_{3}\right)}{\Lambda_{3}}}}{\sqrt{\Lambda_{1}} \Lambda_{2}^{9 / 2} \Lambda_{3}^{9 / 2}\left(\nu_{1}-3 \nu_{2}\right)\left(P_{2}-2 \Lambda_{2}\right)}
$$$$
-\frac{1.014 \beta_{2}^{2} \beta_{3}^{2} G^{4} M_{2} M_{3} P_{1}^{2} P_{2} P_{3}^{2} \sqrt{-\frac{P_{1}\left(P_{1}-2 \Lambda_{1}\right)}{\Lambda_{1}}} \sqrt{-\frac{P_{2}\left(P_{2}-2 \Lambda_{2}\right)}{\Lambda_{2}}} \sqrt{-\frac{P_{3}\left(P_{3}-2 \Lambda_{3}\right)}{\Lambda_{3}}}}{\Lambda_{1}^{5 / 2} \Lambda_{2}^{7 / 2} \Lambda_{3}^{9 / 2}\left(\nu_{1}-3 \nu_{2}\right)\left(P_{2}-2 \Lambda_{2}\right)}
$$$$
+\frac{2.03 \beta_{2}^{2} \beta_{3}^{2} G^{4} M_{2} M_{3} P_{1} P_{2} P_{3}^{2} \sqrt{-\frac{P_{1}\left(P_{1}-2 \Lambda_{1}\right)}{\Lambda_{1}}} \sqrt{-\frac{P_{2}\left(P_{2}-2 \Lambda_{2}\right)}{\Lambda_{2}}} \sqrt{-\frac{P_{3}\left(P_{3}-2 \Lambda_{3}\right)}{\Lambda_{3}}}}{\Lambda_{1}^{3 / 2} \Lambda_{2}^{7 / 2} \Lambda_{3}^{9 / 2}\left(\nu_{1}-3 \nu_{2}\right)\left(P_{2}-2 \Lambda_{2}\right)}
$$

$$
\begin{aligned}
C_{11}^{2} & =-\frac{39.87 \beta_{2}^{2} \beta_{3}^{2} G^{4} M_{2} M_{3} P_{2} P_{3}^{2} \sqrt{-\frac{P_{1}\left(P_{1}-2 \Lambda_{1}\right)}{\Lambda_{1}}} \sqrt{-\frac{P_{2}\left(P_{2}-2 \Lambda_{2}\right)}{\Lambda_{2}}} \sqrt{-\frac{P_{3}\left(P_{3}-2 \Lambda_{3}\right)}{\Lambda_{3}}}}{\sqrt{\Lambda_{1}} \Lambda_{2}^{7 / 2} \Lambda_{3}^{9 / 2}\left(\nu_{1}-3 \nu_{2}\right)\left(P_{2}-2 \Lambda_{2}\right)} \\
+ & \frac{1.69 \beta_{2}^{2} \beta_{3}^{2} G^{4} M_{2} M_{3} P_{1}^{2} P_{3}^{2} \sqrt{-\frac{P_{1}\left(P_{1}-2 \Lambda_{1}\right)}{\Lambda_{1}}} \sqrt{-\frac{P_{2}\left(P_{2}-2 \Lambda_{2}\right)}{\Lambda_{2}}} \sqrt{-\frac{P_{3}\left(P_{3}-2 \Lambda_{3}\right)}{\Lambda_{3}}}}{\Lambda_{1}^{5 / 2} \Lambda_{2}^{5 / 2} \Lambda_{3}^{9 / 2}\left(\nu_{1}-3 \nu_{2}\right)\left(P_{2}-2 \Lambda_{2}\right)} \\
& -\frac{3.38 \beta_{2}^{2} \beta_{3}^{2} G^{4} M_{2} M_{3} P_{1} P_{3}^{2} \sqrt{-\frac{P_{1}\left(P_{1}-2 \Lambda_{1}\right)}{\Lambda_{1}}} \sqrt{-\frac{P_{2}\left(P_{2}-2 \Lambda_{2}\right)}{\Lambda_{2}}} \sqrt{-\frac{P_{3}\left(P_{3}-2 \Lambda_{3}\right)}{\Lambda_{3}}}}{\Lambda_{1}^{3 / 2} \Lambda_{2}^{5 / 2} \Lambda_{3}^{9 / 2}\left(\nu_{1}-3 \nu_{2}\right)\left(P_{2}-2 \Lambda_{2}\right)} \\
+ & \frac{7.41 \beta_{2}^{2} \beta_{3}^{2} G^{4} M_{2} M_{3} P_{3}^{2} \sqrt{-\frac{P_{1}\left(P_{1}-2 \Lambda_{1}\right)}{\Lambda_{1}}} \sqrt{-\frac{P_{2}\left(P_{2}-2 \Lambda_{2}\right)}{\Lambda_{2}}} \sqrt{-\frac{P_{3}\left(P_{3}-2 \Lambda_{3}\right)}{\Lambda_{3}}}}{\sqrt{\Lambda_{1}} \Lambda_{2}^{5 / 2} \Lambda_{3}^{9 / 2}\left(\nu_{1}-3 \nu_{2}\right)\left(P_{2}-2 \Lambda_{2}\right)}
\end{aligned}
$$




$$
\begin{aligned}
& C_{12}^{1}=\frac{2.738 \beta_{2}^{2} \beta_{3}^{2} G^{4} M_{2} M_{3} P_{2}^{3} P_{3}^{2} \sqrt{-\frac{P_{1}\left(P_{1}-2 \Lambda_{1}\right)}{\Lambda_{1}}} \sqrt{-\frac{P_{2}\left(P_{2}-2 \Lambda_{2}\right)}{\Lambda_{2}}} \sqrt{-\frac{P_{3}\left(P_{3}-2 \Lambda_{3}\right)}{\Lambda_{3}}}}{\sqrt{\Lambda_{1}} \Lambda_{2}^{11 / 2} \Lambda_{3}^{9 / 2}\left(\nu_{1}-3 \nu_{2}\right)\left(P_{2}-2 \Lambda_{2}\right)} \\
& -\frac{9.31 \beta_{2}^{2} \beta_{3}^{2} G^{4} M_{2} M_{3} P_{2}^{2} P_{3}^{2} \sqrt{-\frac{P_{1}\left(P_{1}-2 \Lambda_{1}\right)}{\Lambda_{1}}} \sqrt{-\frac{P_{2}\left(P_{2}-2 \Lambda_{2}\right)}{\Lambda_{2}}} \sqrt{-\frac{P_{3}\left(P_{3}-2 \Lambda_{3}\right)}{\Lambda_{3}}}}{\sqrt{\Lambda_{1}} \Lambda_{2}^{9 / 2} \Lambda_{3}^{9 / 2}\left(\nu_{1}-3 \nu_{2}\right)\left(P_{2}-2 \Lambda_{2}\right)} \\
& +\frac{7.667 \beta_{2}^{2} \beta_{3}^{2} G^{4} M_{2} M_{3} P_{2} P_{3}^{2} \sqrt{-\frac{P_{1}\left(P_{1}-2 \Lambda_{1}\right)}{\Lambda_{1}}} \sqrt{-\frac{P_{2}\left(P_{2}-2 \Lambda_{2}\right)}{\Lambda_{2}}} \sqrt{-\frac{P_{3}\left(P_{3}-2 \Lambda_{3}\right)}{\Lambda_{3}}}}{\sqrt{\Lambda_{1}} \Lambda_{2}^{7 / 2} \Lambda_{3}^{9 / 2}\left(\nu_{1}-3 \nu_{2}\right)\left(P_{2}-2 \Lambda_{2}\right)} \\
& -\frac{5.47 \beta_{2}^{2} \beta_{3}^{2} G^{4} M_{2} M_{3} P_{2}^{3} P_{3} \sqrt{-\frac{P_{1}\left(P_{1}-2 \Lambda_{1}\right)}{\Lambda_{1}}} \sqrt{-\frac{P_{2}\left(P_{2}-2 \Lambda_{2}\right)}{\Lambda_{2}}} \sqrt{-\frac{P_{3}\left(P_{3}-2 \Lambda_{3}\right)}{\Lambda_{3}}}}{\sqrt{\Lambda_{1}} \Lambda_{2}^{11 / 2} \Lambda_{3}^{7 / 2}\left(\nu_{1}-3 \nu_{2}\right)\left(P_{2}-2 \Lambda_{2}\right)} \\
& C_{12}^{2}=\frac{18.62 \beta_{2}^{2} \beta_{3}^{2} G^{4} M_{2} M_{3} P_{2}^{2} P_{3} \sqrt{-\frac{P_{1}\left(P_{1}-2 \Lambda_{1}\right)}{\Lambda_{1}}} \sqrt{-\frac{P_{2}\left(P_{2}-2 \Lambda_{2}\right)}{\Lambda_{2}}} \sqrt{-\frac{P_{3}\left(P_{3}-2 \Lambda_{3}\right)}{\Lambda_{3}}}}{\sqrt{\Lambda_{1}} \Lambda_{2}^{9 / 2} \Lambda_{3}^{7 / 2}\left(\nu_{1}-3 \nu_{2}\right)\left(P_{2}-2 \Lambda_{2}\right)} \\
& -\frac{15.33 \beta_{2}^{2} \beta_{3}^{2} G^{4} M_{2} M_{3} P_{2} P_{3} \sqrt{-\frac{P_{1}\left(P_{1}-2 \Lambda_{1}\right)}{\Lambda_{1}}} \sqrt{-\frac{P_{2}\left(P_{2}-2 \Lambda_{2}\right)}{\Lambda_{2}}} \sqrt{-\frac{P_{3}\left(P_{3}-2 \Lambda_{3}\right)}{\Lambda_{3}}}}{\sqrt{\Lambda_{1}} \Lambda_{2}^{7 / 2} \Lambda_{3}^{7 / 2}\left(\nu_{1}-3 \nu_{2}\right)\left(P_{2}-2 \Lambda_{2}\right)} \\
& +\frac{28.35 \beta_{2}^{2} \beta_{3}^{2} G^{4} M_{2} M_{3} P_{2}^{5} \sqrt{-\frac{P_{1}\left(P_{1}-2 \Lambda_{1}\right)}{\Lambda_{1}}} \sqrt{-\frac{P_{2}\left(P_{2}-2 \Lambda_{2}\right)}{\Lambda_{2}}} \sqrt{-\frac{P_{3}\left(P_{3}-2 \Lambda_{3}\right)}{\Lambda_{3}}}}{\sqrt{\Lambda_{1}} \Lambda_{2}^{15 / 2} \Lambda_{3}^{5 / 2}\left(\nu_{1}-3 \nu_{2}\right)\left(P_{2}-2 \Lambda_{2}\right)} \\
& -\frac{131.475 \beta_{2}^{2} \beta_{3}^{2} G^{4} M_{2} M_{3} P_{2}^{4} \sqrt{-\frac{P_{1}\left(P_{1}-2 \Lambda_{1}\right)}{\Lambda_{1}}} \sqrt{-\frac{P_{2}\left(P_{2}-2 \Lambda_{2}\right)}{\Lambda_{2}}} \sqrt{-\frac{P_{3}\left(P_{3}-2 \Lambda_{3}\right)}{\Lambda_{3}}}}{\sqrt{\Lambda_{1}} \Lambda_{2}^{13 / 2} \Lambda_{3}^{5 / 2}\left(\nu_{1}-3 \nu_{2}\right)\left(P_{2}-2 \Lambda_{2}\right)} \\
& \begin{aligned}
C_{13}^{1} & =\frac{167.7 \beta_{2}^{2} \beta_{3}^{2} G^{4} M_{2} M_{3} P_{2}^{3} P_{3}^{2} \sqrt{-\frac{P_{1}\left(P_{1}-2 \Lambda_{1}\right)}{\Lambda_{1}}}}{\sqrt{\Lambda_{1}} \Lambda_{2}^{6} \Lambda_{3}^{4}\left(\nu_{1}-3 \nu_{2}\right)}-\frac{60.97 \beta_{2}^{2} \beta_{3}^{2} G^{4} M_{2} M_{3} P_{2}^{4} P_{3}^{2} \sqrt{-\frac{P_{1}\left(P_{1}-2 \Lambda_{1}\right)}{\Lambda_{1}}}}{\sqrt{\Lambda_{1}} \Lambda_{2}^{7} \Lambda_{3}^{4}\left(\nu_{1}-3 \nu_{2}\right)} \\
& +\frac{12.21 \beta_{2}^{2} \beta_{3}^{2} G^{4} M_{2} M_{3} P_{1} P_{2}^{2} P_{3}^{2} \sqrt{-\frac{P_{1}\left(P_{1}-2 \Lambda_{1}\right)}{\Lambda_{1}}}}{\Lambda_{1}^{3 / 2} \Lambda_{2}^{5} \Lambda_{3}^{4}\left(\nu_{1}-3 \nu_{2}\right)}-\frac{6.10 \beta_{2}^{2} \beta_{3}^{2} G^{4} M_{2} M_{3} P_{1}^{2} P_{2}^{2} P_{3}^{2} \sqrt{-\frac{P_{1}\left(P_{1}-2 \Lambda_{1}\right)}{\Lambda_{1}}}}{\Lambda_{1}^{5 / 2} \Lambda_{2}^{5} \Lambda_{3}^{4}\left(\nu_{1}-3 \nu_{2}\right)}
\end{aligned} \\
& +\frac{6.10 \beta_{2}^{2} \beta_{3}^{2} G^{4} M_{2} M_{3} P_{1}^{2} P_{2} P_{3}^{2} \sqrt{-\frac{P_{1}\left(P_{1}-2 \Lambda_{1}\right)}{\Lambda_{1}}}}{\Lambda_{1}^{5 / 2} \Lambda_{2}^{4} \Lambda_{3}^{4}\left(\nu_{1}-3 \nu_{2}\right)}-\frac{87.76 \beta_{2}^{2} \beta_{3}^{2} G^{4} M_{2} M_{3} P_{2}^{2} P_{3}^{2} \sqrt{-\frac{P_{1}\left(P_{1}-2 \Lambda_{1}\right)}{\Lambda_{1}}}}{\sqrt{\Lambda_{1}} \Lambda_{2}^{5} \Lambda_{3}^{4}\left(\nu_{1}-3 \nu_{2}\right)} \\
& -\frac{34.18 \beta_{2}^{2} \beta_{3}^{2} G^{4} M_{2} M_{3} P_{2} P_{3}^{2} \sqrt{-\frac{P_{1}\left(P_{1}-2 \Lambda_{1}\right)}{\Lambda_{1}}}}{\sqrt{\Lambda_{1}} \Lambda_{2}^{4} \Lambda_{3}^{4}\left(\nu_{1}-3 \nu_{2}\right)}-\frac{12.21 \beta_{2}^{2} \beta_{3}^{2} G^{4} M_{2} M_{3} P_{1} P_{2} P_{3}^{2} \sqrt{-\frac{P_{1}\left(P_{1}-2 \Lambda_{1}\right)}{\Lambda_{1}}}}{\Lambda_{1}^{3 / 2} \Lambda_{2}^{4} \Lambda_{3}^{4}\left(\nu_{1}-3 \nu_{2}\right)}
\end{aligned}
$$




$$
\begin{aligned}
C_{13}^{2} & =\frac{2.03 \beta_{2}^{2} \beta_{3}^{2} G^{4} M_{2} M_{3} P_{1}^{2} P_{3}^{2} \sqrt{-\frac{P_{1}\left(P_{1}-2 \Lambda_{1}\right)}{\Lambda_{1}}}}{\Lambda_{1}^{5 / 2} \Lambda_{2}^{3} \Lambda_{3}^{4}\left(\nu_{1}-3 \nu_{2}\right)}-\frac{4.07 \beta_{2}^{2} \beta_{3}^{2} G^{4} M_{2} M_{3} P_{1} P_{3}^{2} \sqrt{-\frac{P_{1}\left(P_{1}-2 \Lambda_{1}\right)}{\Lambda_{1}}}}{\Lambda_{1}^{3 / 2} \Lambda_{2}^{3} \Lambda_{3}^{4}\left(\nu_{1}-3 \nu_{2}\right)} \\
& +\frac{121.94 \beta_{2}^{2} \beta_{3}^{2} G^{4} M_{2} M_{3} P_{2}^{4} P_{3} \sqrt{-\frac{P_{1}\left(P_{1}-2 \Lambda_{1}\right)}{\Lambda_{1}}}}{\sqrt{\Lambda_{1}} \Lambda_{2}^{7} \Lambda_{3}^{3}\left(\nu_{1}-3 \nu_{2}\right)}+\frac{8.92 \beta_{2}^{2} \beta_{3}^{2} G^{4} M_{2} M_{3} P_{3}^{2} \sqrt{-\frac{P_{1}\left(P_{1}-2 \Lambda_{1}\right)}{\Lambda_{1}}}}{\sqrt{\Lambda_{1}} \Lambda_{2}^{3} \Lambda_{3}^{4}\left(\nu_{1}-3 \nu_{2}\right)}
\end{aligned}
$$

$+\frac{12.22 \beta_{2}^{2} \beta_{3}^{2} G^{4} M_{2} M_{3} P_{1}^{2} P_{2}^{2} P_{3} \sqrt{-\frac{P_{1}\left(P_{1}-2 \Lambda_{1}\right)}{\Lambda_{1}}}}{\Lambda_{1}^{5 / 2} \Lambda_{2}^{5} \Lambda_{3}^{3}\left(\nu_{1}-3 \nu_{2}\right)}-\frac{335.33 \beta_{2}^{2} \beta_{3}^{2} G^{4} M_{2} M_{3} P_{2}^{3} P_{3} \sqrt{-\frac{P_{1}\left(P_{1}-2 \Lambda_{1}\right)}{\Lambda_{1}}}}{\sqrt{\Lambda_{1}} \Lambda_{2}^{6} \Lambda_{3}^{3}\left(\nu_{1}-3 \nu_{2}\right)}$

$+\frac{175.52 \beta_{2}^{2} \beta_{3}^{2} G^{4} M_{2} M_{3} P_{2}^{2} P_{3} \sqrt{-\frac{P_{1}\left(P_{1}-2 \Lambda_{1}\right)}{\Lambda_{1}}}}{\sqrt{\Lambda_{1}} \Lambda_{2}^{5} \Lambda_{3}^{3}\left(\nu_{1}-3 \nu_{2}\right)}-\frac{24.43 \beta_{2}^{2} \beta_{3}^{2} G^{4} M_{2} M_{3} P_{1} P_{2}^{2} P_{3} \sqrt{-\frac{P_{1}\left(P_{1}-2 \Lambda_{1}\right)}{\Lambda_{1}}}}{\Lambda_{1}^{3 / 2} \Lambda_{2}^{5} \Lambda_{3}^{3}\left(\nu_{1}-3 \nu_{2}\right)}$

$$
\begin{aligned}
C_{14}^{1} & =\frac{15.6 \beta_{2}^{2} \beta_{3}^{2} G^{4} M_{2} M_{3} P_{1} P_{2}^{2} P_{3}^{2} \sqrt{-\frac{P_{1}\left(P_{1}-2 \Lambda_{1}\right)}{\Lambda_{1}}}}{\Lambda_{1}^{3 / 2} \Lambda_{2}^{5} \Lambda_{3}^{4}\left(\nu_{1}-3 \nu_{2}\right)}-\frac{7.8 \beta_{2}^{2} \beta_{3}^{2} G^{4} M_{2} M_{3} P_{1}^{2} P_{2}^{2} P_{3}^{2} \sqrt{-\frac{P_{1}\left(P_{1}-2 \Lambda_{1}\right)}{\Lambda_{1}}}}{\Lambda_{1}^{5 / 2} \Lambda_{2}^{5} \Lambda_{3}^{4}\left(\nu_{1}-3 \nu_{2}\right)} \\
& +\frac{10.4 \beta_{2}^{2} \beta_{3}^{2} G^{4} M_{2} M_{3} P_{1}^{2} P_{2} P_{3}^{2} \sqrt{-\frac{P_{1}\left(P_{1}-2 \Lambda_{1}\right)}{\Lambda_{1}}}}{\Lambda_{1}^{5 / 2} \Lambda_{2}^{4} \Lambda_{3}^{4}\left(\nu_{1}-3 \nu_{2}\right)}-\frac{20.8 \beta_{2}^{2} \beta_{3}^{2} G^{4} M_{2} M_{3} P_{1} P_{2} P_{3}^{2} \sqrt{-\frac{P_{1}\left(P_{1}-2 \Lambda_{1}\right)}{\Lambda_{1}}}}{\Lambda_{1}^{3 / 2} \Lambda_{2}^{4} \Lambda_{3}^{4}\left(\nu_{1}-3 \nu_{2}\right)} \\
& +\frac{15.6 \beta_{2}^{2} \beta_{3}^{2} G^{4} M_{2} M_{3} P_{1}^{2} P_{2}^{2} P_{3} \sqrt{-\frac{P_{1}\left(P_{1}-2 \Lambda_{1}\right)}{\Lambda_{1}}}}{\Lambda_{1}^{5 / 2} \Lambda_{2}^{5} \Lambda_{3}^{3}\left(\nu_{1}-3 \nu_{2}\right)}-\frac{31.2 \beta_{2}^{2} \beta_{3}^{2} G^{4} M_{2} M_{3} P_{1} P_{2}^{2} P_{3} \sqrt{-\frac{P_{1}\left(P_{1}-2 \Lambda_{1}\right)}{\Lambda_{1}}}}{\Lambda_{1}^{3 / 2} \Lambda_{2}^{5} \Lambda_{3}^{3}\left(\nu_{1}-3 \nu_{2}\right)} \\
& +\frac{41.6 \beta_{2}^{2} \beta_{3}^{2} G^{4} M_{2} M_{3} P_{1} P_{2} P_{3} \sqrt{-\frac{P_{1}\left(P_{1}-2 \Lambda_{1}\right)}{\Lambda_{1}}}}{\Lambda_{1}^{3 / 2} \Lambda_{2}^{4} \Lambda_{3}^{3}\left(\nu_{1}-3 \nu_{2}\right)}-\frac{20.8 \beta_{2}^{2} \beta_{3}^{2} G^{4} M_{2} M_{3} P_{1}^{2} P_{2} P_{3} \sqrt{-\frac{P_{1}\left(P_{1}-2 \Lambda_{1}\right)}{\Lambda_{1}}}}{\Lambda_{1}^{5 / 2} \Lambda_{2}^{4} \Lambda_{3}^{3}\left(\nu_{1}-3 \nu_{2}\right)}
\end{aligned}
$$

$$
\begin{aligned}
C_{14}^{2} & =\frac{7.8 \beta_{2}^{2} \beta_{3}^{2} G^{4} M_{2} M_{3} P_{1}^{2} P_{2}^{2} P_{3}^{2} \sqrt{-\frac{P_{1}\left(P_{1}-2 \Lambda_{1}\right)}{\Lambda_{1}}}}{\Lambda_{1}^{5 / 2} \Lambda_{2}^{5} \Lambda_{3}^{4}\left(\nu_{2}-2 \nu_{3}\right)}-\frac{15.6 \beta_{2}^{2} \beta_{3}^{2} G^{4} M_{2} M_{3} P_{1} P_{2}^{2} P_{3}^{2} \sqrt{-\frac{P_{1}\left(P_{1}-2 \Lambda_{1}\right)}{\Lambda_{1}}}}{\Lambda_{1}^{3 / 2} \Lambda_{2}^{5} \Lambda_{3}^{4}\left(\nu_{2}-2 \nu_{3}\right)} \\
& +\frac{20.8 \beta_{2}^{2} \beta_{3}^{2} G^{4} M_{2} M_{3} P_{1} P_{2} P_{3}^{2} \sqrt{-\frac{P_{1}\left(P_{1}-2 \Lambda_{1}\right)}{\Lambda_{1}}}}{\Lambda_{1}^{3 / 2} \Lambda_{2}^{4} \Lambda_{3}^{4}\left(\nu_{2}-2 \nu_{3}\right)}-\frac{10.4 \beta_{2}^{2} \beta_{3}^{2} G^{4} M_{2} M_{3} P_{1}^{2} P_{2} P_{3}^{2} \sqrt{-\frac{P_{1}\left(P_{1}-2 \Lambda_{1}\right)}{\Lambda_{1}}}}{\Lambda_{1}^{5 / 2} \Lambda_{2}^{4} \Lambda_{3}^{4}\left(\nu_{2}-2 \nu_{3}\right)} \\
& +\frac{31.2 \beta_{2}^{2} \beta_{3}^{2} G^{4} M_{2} M_{3} P_{1} P_{2}^{2} P_{3} \sqrt{-\frac{P_{1}\left(P_{1}-2 \Lambda_{1}\right)}{\Lambda_{1}}}}{\Lambda_{1}^{3 / 2} \Lambda_{2}^{5} \Lambda_{3}^{3}\left(\nu_{2}-2 \nu_{3}\right)}-\frac{15.6 \beta_{2}^{2} \beta_{3}^{2} G^{4} M_{2} M_{3} P_{1}^{2} P_{2}^{2} P_{3} \sqrt{-\frac{P_{1}\left(P_{1}-2 \Lambda_{1}\right)}{\Lambda_{1}}}}{\Lambda_{1}^{5 / 2} \Lambda_{2}^{5} \Lambda_{3}^{3}\left(\nu_{2}-2 \nu_{3}\right)} \\
+ & \frac{20.8 \beta_{2}^{2} \beta_{3}^{2} G^{4} M_{2} M_{3} P_{1}^{2} P_{2} P_{3} \sqrt{-\frac{P_{1}\left(P_{1}-2 \Lambda_{1}\right)}{\Lambda_{1}}}}{\Lambda_{1}^{5 / 2} \Lambda_{2}^{4} \Lambda_{3}^{3}\left(\nu_{2}-2 \nu_{3}\right)}-\frac{41.6 \beta_{2}^{2} \beta_{3}^{2} G^{4} M_{2} M_{3} P_{1} P_{2} P_{3} \sqrt{-\frac{P_{1}\left(P_{1}-2 \Lambda_{1}\right)}{\Lambda_{1}}}}{\Lambda_{1}^{3 / 2} \Lambda_{2}^{4} \Lambda_{3}^{3}\left(\nu_{2}-2 \nu_{3}\right)}
\end{aligned}
$$




$$
\begin{aligned}
C_{15}^{1}= & \frac{25.61 \beta_{2}^{2} \beta_{3}^{2} G^{4} M_{2} M_{3} P_{2}^{5} P_{3}^{2} \sqrt{-\frac{P_{2}\left(P_{2}-2 \Lambda_{2}\right)}{\Lambda_{2}}}}{\Lambda_{2}^{15 / 2} \Lambda_{3}^{4}\left(\nu_{1}-3 \nu_{2}\right)\left(P_{2}-2 \Lambda_{2}\right)}-\frac{122.38 \beta_{2}^{2} \beta_{3}^{2} G^{4} M_{2} M_{3} P_{2}^{4} P_{3}^{2} \sqrt{-\frac{P_{2}\left(P_{2}-2 \Lambda_{2}\right)}{\Lambda_{2}}}}{\Lambda_{2}^{13 / 2} \Lambda_{3}^{4}\left(\nu_{1}-3 \nu_{2}\right)\left(P_{2}-2 \Lambda_{2}\right)} \\
& \frac{200.3 \beta_{2}^{2} \beta_{3}^{2} G^{4} M_{2} M_{3} P_{2}^{3} P_{3}^{2} \sqrt{-\frac{P_{2}\left(P_{2}-2 \Lambda_{2}\right)}{\Lambda_{2}}}}{\Lambda_{2}^{11 / 2} \Lambda_{3}^{4}\left(\nu_{1}-3 \nu_{2}\right)\left(P_{2}-2 \Lambda_{2}\right)}+\frac{39.84 \beta_{2}^{2} \beta_{3}^{2} G^{4} M_{2} M_{3} P_{1}^{2} P_{2}^{3} P_{3}^{2} \sqrt{-\frac{P_{2}\left(P_{2}-2 \Lambda_{2}\right)}{\Lambda_{2}}}}{\Lambda_{1}^{2} \Lambda_{2}^{11 / 2} \Lambda_{3}^{4}\left(\nu_{1}-3 \nu_{2}\right)\left(P_{2}-2 \Lambda_{2}\right)}
\end{aligned}
$$

$$
\begin{aligned}
& -\frac{31.20 \beta_{2}^{2} \beta_{3}^{2} G^{4} M_{2} M_{3} P_{1} P_{2}^{2} P_{3} \sqrt{-\frac{P_{1}\left(P_{1}-2 \Lambda_{1}\right)}{\Lambda_{1}}}}{\Lambda_{1}^{3 / 2} \Lambda_{2}^{5} \Lambda_{3}^{3}\left(\nu_{1}-3 \nu_{2}\right)}-\frac{79.68 \beta_{2}^{2} \beta_{3}^{2} G^{4} M_{2} M_{3} P_{1} P_{2}^{3} P_{3}^{2} \sqrt{-\frac{P_{2}\left(P_{2}-2 \Lambda_{2}\right)}{\Lambda_{2}}}}{\Lambda_{1} \Lambda_{2}^{11 / 2} \Lambda_{3}^{4}\left(\nu_{1}-3 \nu_{2}\right)\left(P_{2}-2 \Lambda_{2}\right)} \\
& +\frac{239.0 \beta_{2}^{2} \beta_{3}^{2} G^{4} M_{2} M_{3} P_{1} P_{2}^{2} P_{3}^{2} \sqrt{-\frac{P_{2}\left(P_{2}-2 \Lambda_{2}\right)}{\Lambda_{2}}}}{\Lambda_{1} \Lambda_{2}^{9 / 2} \Lambda_{3}^{4}\left(\nu_{1}-3 \nu_{2}\right)\left(P_{2}-2 \Lambda_{2}\right)}-\frac{119.5 \beta_{2}^{2} \beta_{3}^{2} G^{4} M_{2} M_{3} P_{1}^{2} P_{2}^{2} P_{3}^{2} \sqrt{-\frac{P_{2}\left(P_{2}-2 \Lambda_{2}\right)}{\Lambda_{2}}}}{\Lambda_{1}^{2} \Lambda_{2}^{9 / 2} \Lambda_{3}^{4}\left(\nu_{1}-3 \nu_{2}\right)\left(P_{2}-2 \Lambda_{2}\right)}
\end{aligned}
$$

$$
\begin{aligned}
C_{15}^{2} & =\frac{5.17 \beta_{2}^{2} \beta_{3}^{2} G^{4} M_{2} M_{3} P_{2} P_{3}^{2} \sqrt{-\frac{P_{2}\left(P_{2}-2 \Lambda_{2}\right)}{\Lambda_{2}}}}{\Lambda_{2}^{7 / 2} \Lambda_{3}^{4}\left(\nu_{1}-3 \nu_{2}\right)\left(P_{2}-2 \Lambda_{2}\right)}-\frac{5.19 \beta_{2}^{2} \beta_{3}^{2} G^{4} M_{2} M_{3} P_{1}^{4} P_{2} P_{3}^{2} \sqrt{-\frac{P_{2}\left(P_{2}-2 \Lambda_{2}\right)}{\Lambda_{2}}}}{\Lambda_{1}^{4} \Lambda_{2}^{7 / 2} \Lambda_{3}^{4}\left(\nu_{1}-3 \nu_{2}\right)\left(P_{2}-2 \Lambda_{2}\right)} \\
& +\frac{53.3 \beta_{2}^{2} \beta_{3}^{2} G^{4} M_{2} M_{3} P_{1}^{2} P_{2} P_{3}^{2} \sqrt{-\frac{P_{2}\left(P_{2}-2 \Lambda_{2}\right)}{\Lambda_{2}}}}{\Lambda_{1}^{2} \Lambda_{2}^{7 / 2} \Lambda_{3}^{4}\left(\nu_{1}-3 \nu_{2}\right)\left(P_{2}-2 \Lambda_{2}\right)}+\frac{20.8 \beta_{2}^{2} \beta_{3}^{2} G^{4} M_{2} M_{3} P_{1}^{3} P_{2} P_{3}^{2} \sqrt{-\frac{P_{2}\left(P_{2}-2 \Lambda_{2}\right)}{\Lambda_{2}}}}{\Lambda_{1}^{3} \Lambda_{2}^{7 / 2} \Lambda_{3}^{4}\left(\nu_{1}-3 \nu_{2}\right)\left(P_{2}-2 \Lambda_{2}\right)}
\end{aligned}
$$

$+\frac{16.90 \beta_{2}^{2} \beta_{3}^{2} G^{4} M_{2} M_{3} P_{3}^{2} \sqrt{-\frac{P_{2}\left(P_{2}-2 \Lambda_{2}\right)}{\Lambda_{2}}}}{\Lambda_{2}^{5 / 2} \Lambda_{3}^{4}\left(\nu_{1}-3 \nu_{2}\right)\left(P_{2}-2 \Lambda_{2}\right)}-\frac{148.0 \beta_{2}^{2} \beta_{3}^{2} G^{4} M_{2} M_{3} P_{1} P_{2} P_{3}^{2} \sqrt{-\frac{P_{2}\left(P_{2}-2 \Lambda_{2}\right)}{\Lambda_{2}}}}{\Lambda_{1} \Lambda_{2}^{7 / 2} \Lambda_{3}^{4}\left(\nu_{1}-3 \nu_{2}\right)\left(P_{2}-2 \Lambda_{2}\right)}$

$+\frac{7.3 \beta_{2}^{2} \beta_{3}^{2} G^{4} M_{2} M_{3} P_{1}^{4} P_{3}^{2} \sqrt{-\frac{P_{2}\left(P_{2}-2 \Lambda_{2}\right)}{\Lambda_{2}}}}{\Lambda_{1}^{4} \Lambda_{2}^{5 / 2} \Lambda_{3}^{4}\left(\nu_{1}-3 \nu_{2}\right)\left(P_{2}-2 \Lambda_{2}\right)}-\frac{29.0 \beta_{2}^{2} \beta_{3}^{2} G^{4} M_{2} M_{3} P_{1}^{3} P_{3}^{2} \sqrt{-\frac{P_{2}\left(P_{2}-2 \Lambda_{2}\right)}{\Lambda_{2}}}}{\Lambda_{1}^{3} \Lambda_{2}^{5 / 2} \Lambda_{3}^{4}\left(\nu_{1}-3 \nu_{2}\right)\left(P_{2}-2 \Lambda_{2}\right)}$

$$
\begin{aligned}
C_{16}^{1} & =\frac{13.19 \beta_{2}^{2} \beta_{3}^{2} G^{4} M_{2} M_{3} P_{2}^{4} P_{3}^{2} \sqrt{-\frac{P_{1}\left(P_{1}-2 \Lambda_{1}\right)}{\Lambda_{1}}}}{\sqrt{\Lambda_{1}} \Lambda_{2}^{7} \Lambda_{3}^{4}\left(\nu_{1}-3 \nu_{2}\right)}-\frac{32.98 \beta_{2}^{2} \beta_{3}^{2} G^{4} M_{2} M_{3} P_{2}^{3} P_{3}^{2} \sqrt{-\frac{P_{1}\left(P_{1}-2 \Lambda_{1}\right)}{\Lambda_{1}}}}{\sqrt{\Lambda_{1}} \Lambda_{2}^{6} \Lambda_{3}^{4}\left(\nu_{1}-3 \nu_{2}\right)} \\
& +\frac{19.79 \beta_{2}^{2} \beta_{3}^{2} G^{4} M_{2} M_{3} P_{2} P_{3}^{2} \sqrt{-\frac{P_{1}\left(P_{1}-2 \Lambda_{1}\right)}{\Lambda_{1}}}}{\sqrt{\Lambda_{1}} \Lambda_{2}^{4} \Lambda_{3}^{4}\left(\nu_{1}-3 \nu_{2}\right)}+\frac{3.29 \beta_{2}^{2} \beta_{3}^{2} G^{4} M_{2} M_{3} P_{2}^{2} P_{3}^{2} \sqrt{-\frac{P_{1}\left(P_{1}-2 \Lambda_{1}\right)}{\Lambda_{1}}}}{\sqrt{\Lambda_{1}} \Lambda_{2}^{5} \Lambda_{3}^{4}\left(\nu_{1}-3 \nu_{2}\right)}
\end{aligned}
$$

$+\frac{65.96 \beta_{2}^{2} \beta_{3}^{2} G^{4} M_{2} M_{3} P_{2}^{3} P_{3} \sqrt{-\frac{P_{1}\left(P_{1}-2 \Lambda_{1}\right)}{\Lambda_{1}}}}{\sqrt{\Lambda_{1}} \Lambda_{2}^{6} \Lambda_{3}^{3}\left(\nu_{1}-3 \nu_{2}\right)}-\frac{26.38 \beta_{2}^{2} \beta_{3}^{2} G^{4} M_{2} M_{3} P_{2}^{4} P_{3} \sqrt{-\frac{P_{1}\left(P_{1}-2 \Lambda_{1}\right)}{\Lambda_{1}}}}{\sqrt{\Lambda_{1}} \Lambda_{2}^{7} \Lambda_{3}^{3}\left(\nu_{1}-3 \nu_{2}\right)}$

$-\frac{39.58 \beta_{2}^{2} \beta_{3}^{2} G^{4} M_{2} M_{3} P_{2} P_{3} \sqrt{-\frac{P_{1}\left(P_{1}-2 \Lambda_{1}\right)}{\Lambda_{1}}}}{\sqrt{\Lambda_{1}} \Lambda_{2}^{4} \Lambda_{3}^{3}\left(\nu_{1}-3 \nu_{2}\right)}-\frac{6.59 \beta_{2}^{2} \beta_{3}^{2} G^{4} M_{2} M_{3} P_{2}^{2} P_{3} \sqrt{-\frac{P_{1}\left(P_{1}-2 \Lambda_{1}\right)}{\Lambda_{1}}}}{\sqrt{\Lambda_{1}} \Lambda_{2}^{5} \Lambda_{3}^{3}\left(\nu_{1}-3 \nu_{2}\right)}$ 


$$
\begin{aligned}
C_{16}^{2} & =\frac{32.98 \beta_{2}^{2} \beta_{3}^{2} G^{4} M_{2} M_{3} P_{2}^{3} P_{3}^{2} \sqrt{-\frac{P_{1}\left(P_{1}-2 \Lambda_{1}\right)}{\Lambda_{1}}}}{\sqrt{\Lambda_{1}} \Lambda_{2}^{6} \Lambda_{3}^{4}\left(\nu_{2}-2 \nu_{3}\right)}-\frac{13.19 \beta_{2}^{2} \beta_{3}^{2} G^{4} M_{2} M_{3} P_{2}^{4} P_{3}^{2} \sqrt{-\frac{P_{1}\left(P_{1}-2 \Lambda_{1}\right)}{\Lambda_{1}}}}{\sqrt{\Lambda_{1}} \Lambda_{2}^{7} \Lambda_{3}^{4}\left(\nu_{2}-2 \nu_{3}\right)} \\
& -\frac{19.79 \beta_{2}^{2} \beta_{3}^{2} G^{4} M_{2} M_{3} P_{2} P_{3}^{2} \sqrt{-\frac{P_{1}\left(P_{1}-2 \Lambda_{1}\right)}{\Lambda_{1}}}}{\sqrt{\Lambda_{1}} \Lambda_{2}^{4} \Lambda_{3}^{4}\left(\nu_{2}-2 \nu_{3}\right)}-\frac{3.29 \beta_{2}^{2} \beta_{3}^{2} G^{4} M_{2} M_{3} P_{2}^{2} P_{3}^{2} \sqrt{-\frac{P_{1}\left(P_{1}-2 \Lambda_{1}\right)}{\Lambda_{1}}}}{\sqrt{\Lambda_{1}} \Lambda_{2}^{5} \Lambda_{3}^{4}\left(\nu_{2}-2 \nu_{3}\right)} \\
& +\frac{26.38 \beta_{2}^{2} \beta_{3}^{2} G^{4} M_{2} M_{3} P_{2}^{4} P_{3} \sqrt{-\frac{P_{1}\left(P_{1}-2 \Lambda_{1}\right)}{\Lambda_{1}}}}{\sqrt{\Lambda_{1}} \Lambda_{2}^{7} \Lambda_{3}^{3}\left(\nu_{2}-2 \nu_{3}\right)}-\frac{65.96 \beta_{2}^{2} \beta_{3}^{2} G^{4} M_{2} M_{3} P_{2}^{3} P_{3} \sqrt{-\frac{P_{1}\left(P_{1}-2 \Lambda_{1}\right)}{\Lambda_{1}}}}{\sqrt{\Lambda_{1}} \Lambda_{2}^{6} \Lambda_{3}^{3}\left(\nu_{2}-2 \nu_{3}\right)} \\
+ & \frac{39.58 \beta_{2}^{2} \beta_{3}^{2} G^{4} M_{2} M_{3} P_{2} P_{3} \sqrt{-\frac{P_{1}\left(P_{1}-2 \Lambda_{1}\right)}{\Lambda_{1}}}}{\sqrt{\Lambda_{1}} \Lambda_{2}^{4} \Lambda_{3}^{3}\left(\nu_{2}-2 \nu_{3}\right)}+\frac{6.59 \beta_{2}^{2} \beta_{3}^{2} G^{4} M_{2} M_{3} P_{2}^{2} P_{3} \sqrt{-\frac{P_{1}\left(P_{1}-2 \Lambda_{1}\right)}{\Lambda_{1}}}}{\sqrt{\Lambda_{1}} \Lambda_{2}^{5} \Lambda_{3}^{3}\left(\nu_{2}-2 \nu_{3}\right)}
\end{aligned}
$$

Na tabela (4.3) mostramos os coeficientes $C_{1}, \ldots, C_{16}$, em séries de potência da excentricidade do planeta teste, para valores fixos das excentricidades $e_{1}=0.05$ e $e_{3}=0.05$. 


\begin{tabular}{|c|c|c|}
\hline Número & Multipleto & Coeficiente \\
\hline \hline 1 & $\lambda_{1}-2 \lambda_{2}-2 \lambda_{3}+3 \varpi_{1}$ & $2909.08 e_{2}^{2}-1398.79$ \\
\hline 2 & $\lambda_{1}-2 \lambda_{2}-2 \lambda_{3}+2 \varpi_{1}+\varpi_{2}$ & $1.41067 \times 10^{8} e_{2}^{3}-3.62058 \times 10^{7} e_{2}$ \\
\hline 3 & $\lambda_{1}-2 \lambda_{2}-2 \lambda_{3}+\varpi_{1}+2 \varpi_{2}$ & $-7.52347 \times 10^{6} e_{2}^{2}$ \\
\hline 4 & $\lambda_{1}-2 \lambda_{2}-2 \lambda_{3}-\varpi_{1}+4 \varpi_{2}$ & $-2.8728672996686 \times 10^{-13} e_{2}^{2}$ \\
\hline 5 & $\lambda_{1}-2 \lambda_{2}-2 \lambda_{3}+3 \varpi_{1}+\varpi_{2}-\varpi_{3}$ & $448.229 e_{2}^{3}-239.055 e_{2}$ \\
\hline 6 & $\lambda_{1}-2 \lambda_{2}-2 \lambda_{3}+2 \varpi_{1}+2 \varpi_{2}-\varpi_{3}$ & $-6.18975 \times 10^{6} e_{2}^{2}$ \\
\hline 7 & $\lambda_{1}-2 \lambda_{2}-2 \lambda_{3}+\varpi_{1}+3 \varpi_{2}-\varpi_{3}$ & $-1.01171 \times 10^{6} e_{2}^{3}$ \\
\hline 8 & $\lambda_{1}-2 \lambda_{2}-2 \lambda_{3}-\varpi_{1}+5 \varpi_{2}-\varpi_{3}$ & $1.149146919867441 \times 10^{-11} e_{2}^{3}$ \\
\hline 9 & $\lambda_{1}-2 \lambda_{2}-2 \lambda_{3}+2 \varpi_{1}+\varpi_{3}$ & $-1.14192 \times 10^{6} e_{2}^{2}-513.26$ \\
\hline 10 & $\lambda_{1}-2 \lambda_{2}-2 \lambda_{3}+3 \varpi_{1}-\varpi_{2}+\varpi_{3}$ & $228.815 e_{2}-859.951 e_{2}^{3}$ \\
\hline 11 & $\lambda_{1}-2 \lambda_{2}-2 \lambda_{3}+\varpi_{1}+\varpi_{2}+\varpi_{3}$ & $75534.1 e_{2}^{3}+413895 . e_{2}$ \\
\hline 12 & $\lambda_{1}-2 \lambda_{2}-2 \lambda_{3}-\varpi_{1}+3 \varpi_{2}+\varpi_{3}$ & $429399 . e_{2}^{3}$ \\
\hline 13 & $\lambda_{1}-2 \lambda_{2}-2 \lambda_{3}+\varpi_{1}+2 \varpi_{3}$ & $22306 . e_{2}^{2}-11643.2$ \\
\hline 14 & $\lambda_{1}-2 \lambda_{2}-2 \lambda_{3}+3 \varpi_{1}-2 \varpi_{2}+2 \varpi_{3}$ & $16.963 e_{2}^{2}$ \\
\hline 15 & $\lambda_{1}-2 \lambda_{2}-2 \lambda_{3}+2 \varpi_{1}-\varpi_{2}+2 \varpi_{3}$ & $87577 . e_{2}^{3}+220057 . e_{2}$ \\
\hline 16 & $\lambda_{1}-2 \lambda_{2}-2 \lambda_{3}-\varpi_{1}+2 \varpi_{2}+2 \varpi_{3}$ & $-12909.2 e_{2}^{2}$ \\
\hline
\end{tabular}

Tabela 4.3 - Coeficientes dos multipletos em série de potência da excentricidade do planeta teste $e_{2}$ até terceira ordem. Fixos $e_{1}=0.05, e_{3}=0.05$ 
Capítulo 5

\section{Alguns Tópicos da Teoria da Difusão}

Neste capítulo apresentaremos, de forma sumária, alguns tópicos da teoria da difusão que serão importantes no estudo da caoticidade dos muitos sistemas ressonantes.

\subsection{Conceitos básicos}

De forma geral, em um sistema com $N$ graus de liberdade, trajetórias estocásticas e regulares estão intimamente próximas no espaço de fase de dimensão $2 N$, e na superfície de seção de dimensão $(2 N-2)$. Camadas estocásticas, no espaço de fase, existem próximas às ressonâncias do movimento. A espessura das camadas aumenta com o crescimento da perturbação, levando a um overlap das ressonâncias primárias, movimentos através das camadas e ao aparecimento de uma forte estocasticidade no movimento. No limite, para uma pequena perturbação, contudo, não ocorre overlap das ressonâncias primárias. Um novo comportamento físico para o movimento aparece: o movimento ao longo da camada ressonante, chamado difusão de Arnold (ver Lichtenberg e Lieberman (1992)).

\subsubsection{Aspecto Geométrico da Difusão}

É importante termos em mente o que entendemos por difusão. De uma maneira formal, temos o seguinte: dados dois pontos $A$ e $B$ do espaço de fase, podemos encontrar uma órbita $x(t)$ com condições iniciais $x(0)$ numa pequena vizinhança de $A$ e $x(T)$ numa pequena vizinhança de $B$ após um tempo $T$ ?

De maneira geral tal órbita, se existe, é caótica, ou seja pequenas mudanças nas condições iniciais $x(0)$ geram grandes mudanças na órbita $x(t)$. De fato, na presença de caos as 
órbitas exibem comportamentos topológico e estatístico bastante complexo, e neste caso a transferência no espaço de fase é chamada Difusão Caótica.

Devemos notar que mesmo existindo movimentos caóticos em determinada região do espaço de fase, não podemos afirmar que o sistema é globalmente instável. De fato, a região onde ocorre o caos pode se localizar em uma pequena região do espaço de fase. Para ver isso, consideremos o mapa (ver, Lega et al. (2010))

$$
\begin{aligned}
\varphi^{\prime} & =\varphi+I \\
I^{\prime} & =I+\epsilon \frac{\sin \varphi^{\prime}}{\left(\cos \varphi^{\prime}+c-1\right)^{2}}
\end{aligned}
$$

Na figura (5.1)( lado esquerdo), podemos ver que o mapa, no plano $(I, \varphi)$, tem muitos toros invariantes que funcionam como " barreiras" topológicas que impedem a difusão na ação $I$. Por exemplo, as regiões cujas ações estão em torno de $I=0.31$ e $I=0.36$, estão separadas por curvas invariantes. Assim, não existem curvas que possam conectar estas diferentes regiões caóticas. Se aumentarmos o valor de $\epsilon$ (lado direito), veremos que as curvas invariantes que funcionavam como barreiras topológicas desaparecem, isso faz com que possa haver, a princípio, difusão das órbitas.
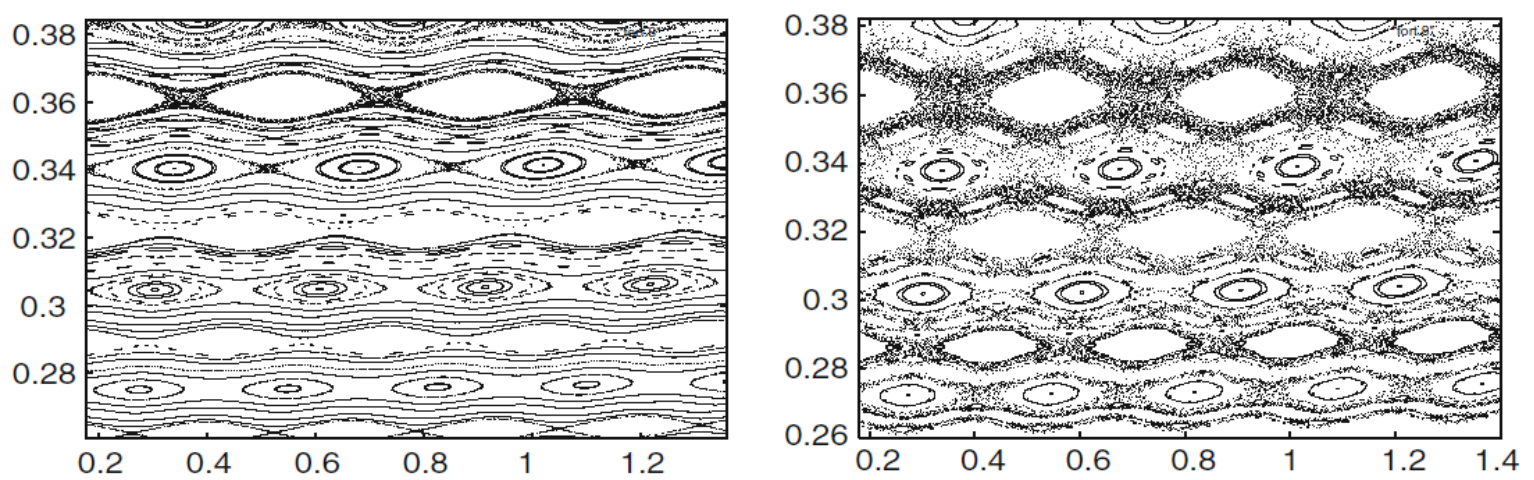

Figura 5.1: Retrato de fase do mapa para $\epsilon=0.002$ e $\epsilon=0.0026$, figura da esquerda e figura da direita, respectivamente

\subsubsection{Overlaps De Ressonâncias}

Num sistema sob a ação de várias ressonâncias pode acontecer overlap. Cada ressonância determina o seu domínio no espaço de fase, contudo o movimento em uma vizin- 
hança de determinada ressonância será afetado pela presença da outra. Por outro lado, se as ressonâncias estiverem suficientemente distantes uma da outra e se considerarmos perturbações suficientemente fracas, o movimento pode ser confinado na vizinhança de uma ou de outra ressonância, de acordo com as condições iniciais escolhidas. Assim, em cada ressonância o sistema se comporta como um pêndulo perturbado pela interação de uma ressonância com a outra(ver, (Chirikov, 1979)). Este fato é usado no capítulo (6).

\subsubsection{Difusão na Astronomia}

O problema da estabilidade do Sistema Solar tem exigido, durante séculos, de matemáticos e astrônomos bastante trabalho para provar teoremas a respeito de estabilidade para sistemas dinâmicos que podem ser usados como boa aproximação para nosso sistema solar, de uma forma geral, para o problema de $N$ corpos. Sabemos que no cinturão de asteroides existem os chamados Kirkwood gaps (Wisdom, 1982), regiões de ressonâncias de movimentos médios desprovidas de asteroides. Estes gaps estão associados a ressonâncias de movimentos médios com Júpiter que causa um rápido crescimento da excentricidade do asteroide (ver, Wisdom (1983)). Por outro lado, tem sido constatado a existência de difusão caótica no Sistema Solar, ou seja, a lenta evolução das excentricidades e inclinações. Este fenômeno está associado à ressonâncias de movimentos médios de altas ordens, estas ressonâncias surgem em sistemas que são perturbações do problema de Kepler.

\subsection{Difusão em um Passeio Aleatório}

Para maiores detalhes dos conceitos apresentados nesta seção, em particular os conceitos abordados nas subseções (5.2.3) e (5.2.4) ver (Chandrasekhar (1943)) e (Varvoglis (2005)) (ver também, Tomé (2001)).

\subsubsection{Variáveis Aleatórias}

Quando estudamos funções, de um modo geral, estamos interessados em certos conjuntos, seu domínio de definição, contra domínio e imagem. Na teoria das probabilidades um dos principais conceitos é o de variáveis aleatórias. Consideremos uma variável aleatória $X$, seu domínio é chamado espaço amostral, denotado por $S$. Seu contradomínio é a reta 
real $\mathbb{R}$, e o valor que a variável $X$ assume é denotado por $x$. Simbolicamente,

$$
X: S \rightarrow \mathbb{R}
$$

Como exemplo, suponha que $S=\{$ cara,coroa $\}$. Devemos notar que podemos definir infinitas variáveis randômicas para este mesmo espaço amostral, como por exemplo

$$
X(S)=\{-l, l\}
$$

\subsubsection{Funções de Probabilidade}

Começamos aqui com a seguinte pergunta:

Qual a probabilidade de que a variável aleatória, definida pela equação (5.3), assuma o valor $l$ em um passo.

Isto é dado pela função de probabilidade $f_{D}(x)$

$$
f_{D}(l)=p, \quad f_{D}(-l)=1-p
$$

Observe que a variável aleatória é "mapeada" em um número real, que por sua vez é levado a uma probabilidade:

$$
X \rightarrow x \rightarrow f_{D}(x)
$$

por outro lado espaço amostral é levado na reta real, que por sua vez é levada no intervalo $[0,1]$.

A função $f_{D}$, é tal que

$$
\begin{aligned}
f_{D} & \geq 0 \\
\sum f_{D_{i}} & =1 .
\end{aligned}
$$

Notemos que o subscrito $D$ indica que esta forma da função de probabilidade é válida para variáveis aleatórias discretas. No caso em que temos variáveis aleatórias contínuas, a probabilidade de que a variável aleatória $X$ assuma um valor no intervalo $[x, x+d x]$ é dada por 


$$
d P(x)=f_{C}(x) d x
$$

onde $f_{C}$ é a função densidade de probabilidade. De forma análoga, a função $f_{C}$ deve ser não-negativa e tal que

$$
\int_{S} f_{C}(x) d x=1
$$

O estudo da função $f$ é, em geral, feito calculando-se a média

$$
\begin{aligned}
& E(X)=\langle x\rangle=\mu=\sum_{S} x_{i} f_{D}\left(x_{i}\right), \text { caso discreto } \\
& E(X)=\langle x\rangle=\mu=\int_{S} x f_{C}(x) d x, \text { caso contínuo }
\end{aligned}
$$

e a variância

$$
\begin{array}{r}
\operatorname{Var}(X)=\sigma^{2}=E\left[(X-\mu)^{2}\right]=\sum_{S}\left(x_{i}-\mu\right)^{2} f_{D}\left(x_{i}\right), \text { caso discreto } \\
\operatorname{Var}(X)=\sigma^{2}=E\left[(X-\mu)^{2}\right]=\int_{S}(x-\mu)^{2} f_{C}(x) d x, \text { caso contínuo. }
\end{array}
$$

Temos uma importante relação entre a média e a variância

$$
\sigma^{2}=E\left[(X-\mu)^{2}\right]=E\left(x^{2}\right)-2 \mu E(X)+\mu^{2}=\left\langle x^{2}\right\rangle-\langle x\rangle^{2}
$$

Teorema 2. Sejam $X_{1}, X_{2}, \ldots, X_{n}$ variáveis aleatórias, com funções de distribuição tais que $\mu_{i}=\mu$ e $\sigma_{i}=\sigma$, todas com mesmos $\sigma$ e $\mu$. Definamos uma nova variável aleatória, $S_{N}$, da seguinte forma

$$
S_{N}=X_{1}+X_{2}+\ldots+X_{N}
$$

Então, o teorema nos diz que, para $N \rightarrow \infty$, a função densidade de probabilidade, $f\left(S_{N}\right)$, da nova variável randômica é Gaussiana, com $\mu_{S_{N}}=N \mu$ e $\sigma_{S_{N}}^{2}=N \sigma^{2}$

Claramente, se as variáveis $X_{i}$ tem distribuições Gaussianas, então o resultado é verdadeiro e o teorema do limite central não é necessário. 


\subsubsection{Passeio Aleatório}

Consideremos uma partícula sofrendo deslocamentos ao longo de uma reta, onde cada deslocamento corresponde a um passo (ver, (Chandrasekhar (1943)), (Varvoglis (2005)) e (Tomé (2001))). Suponhamos também que cada passo tenha o mesmo comprimento e que cada passo possa ser para frente ou para trás com a mesma probabilidade 1/2. Claramente, após $N$ passos a partícula pode estar em qualquer um dos pontos abaixo

$$
-N,-N+1, \ldots,-1,0,1, \ldots, N-1, N
$$

Podemos pensar esses pontos como coordenadas ao longo da reta, se usarmos a unidade comprimento como sendo igual ao comprimento de um passo.

Vamos supor que a partícula saia da "origem " $(\mathrm{x}=0)$, e associamos a i-ésima variável aleatória ao i-ésimo passo,

$$
X_{i}: S \rightarrow \mathbb{R}
$$

Note que se definirmos $S_{N}=X_{1}+X_{2}+\ldots+X_{N}$, então $S_{N}$ nos dará a posição da partícula após $N$ passos. Usando o teorema (2), sabemos que após um determinado número de passos, a distância à origem obedecerá a uma distribuição Gaussiana.

Aqui surge a seguinte pergunta: Qual a probabilidade $P(N, m)$ que a partícula chegue a posição(ponto final) $m$ após $N$ passos( $N$ deslocamentos)?

Devemos notar que cada passo individual pode ser para frente ou para trás independentemente da direção de todos os passos precedentes. Portanto, todas as possíveis sequências de passos, cada uma tomada numa direção definida, tem a mesma probabilidade. Dito de outra forma, a probabilidade de qualquer sequência de $N$ passos é $\left(\frac{1}{2}\right)^{N}$. Portanto, a probabilidade $P(N, m)$ é o produto de $\left(\frac{1}{2}\right)^{N}$ pelo número de sequências distintas que levarão ao ponto $m$ após $N$ passos.

Como um exemplo, consideremos $\{-l, l\}=\{x\}$ e $f_{D}(l)=f_{D}(-l)=1 / 2$. Note que, neste caso, $\mu_{S_{N}}=E\left(S_{N}\right)=0$ e $\sigma_{S_{N}}^{2}=\operatorname{Var}\left(S_{N}\right)=N l^{2}$. De fato, $\mu_{S_{N}}=N \cdot E\left(X_{i}\right)$, mas $E\left(X_{i}\right)=\mu_{i}=\sum x_{i} \cdot f_{D}\left(x_{i}\right)=\frac{1}{2} l+\frac{1}{2}(-l)=0$. Donde, $\mu_{S_{N}}=0$. Além disso,

$$
\operatorname{Var}\left(S_{N}\right)=\sigma_{S_{N}}^{2}=E\left[\left(S_{N}-\mu_{S_{N}}\right)\right]=N \sigma_{X_{i}}^{2}
$$

como 


$$
\sigma_{X_{i}}^{2}=\sum\left(x_{i}-\mu_{i}\right)^{2} f_{D}\left(x_{i}\right)=\frac{1}{2} l^{2}+\frac{1}{2}(-l)^{2} .
$$

Tem-se

$$
\sigma_{S_{N}}^{2}=\operatorname{Var}\left(S_{N}\right)=N l^{2}
$$

O que queremos saber de fato é qual a probabilidade $P(N, m)$, de que uma partícula, saindo da origem, esteja à uma distância $y=m l$. Na tabela (5.1) mostramos, graficamente, qual é essa probabilidade.

Note que para chegar à posição $m$ após $N$ passos, a partícula deve ter tomado $(N+$ $m) / 2$ passo para frente(direção positiva) e $(N-m) / 2$ passos para trás(direção negativa). Notemos também que, seja necessário que $m$ e $N$ tenham a mesma paridade.

O número de sequências distintas, ou seja, a maneira de chegar a posição $m$ é dado por

$$
\left(\begin{array}{c}
(N+m) / 2+(N-m) / 2 \\
(N+m) / 2
\end{array}\right)=\frac{N !}{[(N+m) / 2] ![(N-m) / 2] !} .
$$

Como cada uma maneira tem a probabilidade de $(1 / 2)^{N}$, segue que a probabilidade procurada é dada por

$$
P(N, m)=\frac{N !}{[(N+m) / 2] ![(N-m) / 2] !}\left(\frac{1}{2}\right)^{N} .
$$

A distribuição (5.17) acima é uma distribuição de Bernoulli.

Usando o teorema (2), temos que no limite $N>>1$ a distribuição de Bernoulli torna-se Gaussiana. De fato, no limite para $N \rightarrow \infty$

$$
P(N, m)=\frac{2}{\sqrt{\pi N}} \exp \left[-\frac{m^{2}}{2 N}\right]
$$

Para $N$ e $m$ muito grande podemos assumir que estas variáveis são contínuas. Assim, fazendo $y=m l$, temos que a probabilidade para chegar no intervalo $[y, y+\Delta y]$ no "tempo" $N$ é dada por

$$
P(N, y) \Delta y=P(N, m)\left(\frac{\Delta y}{2 l}\right) .
$$

Note que como $m$ é par ou ímpar, dependendo se $N$ é par ou ímpar respectivamente, temos "dois tempos". Assim, o "tempo" $N$ sempre cresce por dois passos. Portanto, usando a equação (5.19) a (5.18) torna-se 


$$
P(N, m)=\frac{2}{\sqrt{\pi N l^{2}}} \exp \left[-\frac{y^{2}}{2 N l^{2}}\right]
$$

Agora suponhamos que a partícula sofre $n$ deslocamentos por unidade de tempo, tomemos $N=n t$ e definamos

$$
D=\frac{1}{2} n l^{2}
$$

Então, a probabilidade da partícula chegar no intervalo $[y, \Delta y]$ no tempo $t$ é dada por

$$
d P(y, t)=f(y, t) d y
$$

onde $f(y, t)$ é função densidade de probabilidade

$$
f(y, t)=\frac{1}{2 \sqrt{\pi D t}} \exp \left[-\frac{y^{2}}{4 D t}\right] .
$$

Observe que a equação (5.23) nos diz, que no limite, um caminho aleatório em uma dimensão leva naturalmente a funções contínuas de duas variáveis, a posição $y$, e o tempo $t$.

\subsubsection{Passeio aleatório com Barreiras}

Agora continuaremos a discutir o problema do caminho aleatório unidimensional, mas impondo certas restrições ao movimento da partícula, através da presença de barreira refletora ou absorvente (ver, Chandrasekhar (1943), Varvoglis (2005)).

\subsubsection{Barreira Refletora em $m=m_{1}$}

Sem perda de generalidade, podemos supor $m_{1}>0$. Colocando-se uma barreira refletora, o que acontece é que sempre que a partícula chega em $m_{1}$ ela tem a possibilidade de refazer seu passo para $m_{1}-1$. Qual a densidade de probabilidade neste caso? Para discutirmos melhor essa questão, consideremos o curso da partícula no plano $(\mathrm{m}, \mathrm{N})$ como na figura (5.2)

Pela figura, o deslocamento da partícula por um passo significa que o ponto que a representa move-se para cima por uma unidade, enquanto ao mesmo tempo ele sofre um deslocamento lateral de uma unidade, quer na direção positiva quer na direção negativa. Sem uma " 


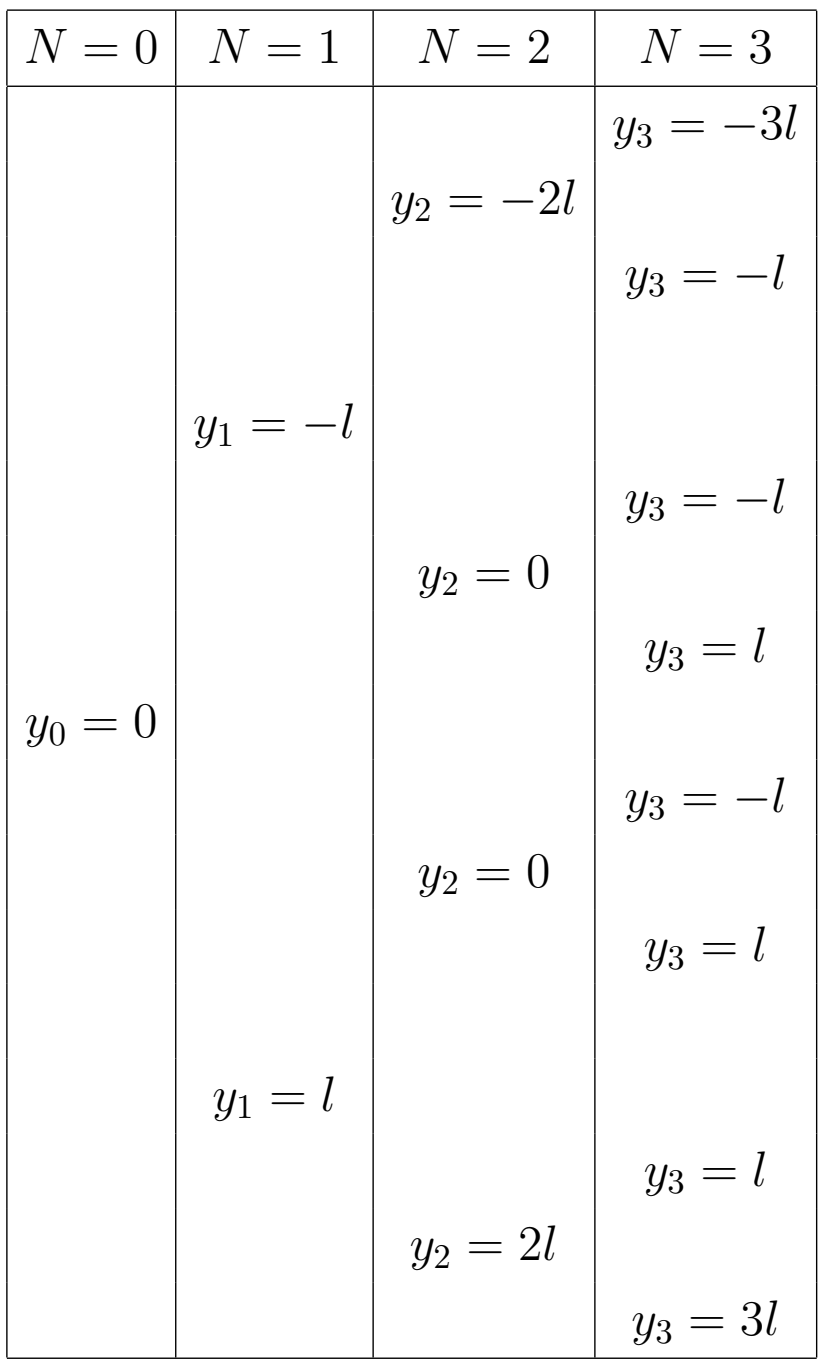

Tabela 5.1 - Caminho aleatório com unidade de um passo igual $l$

parede" que reflita a partícula em $m=m_{1}$, a probabilidade dela chegar em $m$ (ponto final) após $N$ passos é dada pela equação (5.17). Porém com a presença de uma "parede" refletora devemos levar em conta o fato de que um caminho atingindo $m$, após $n$ reflexões, deve ser contado $2^{n}$ vezes, desde que em cada reflexão a partícula tem a possibilidade de refazer seu passos. Notemos que uma trajetória que chega em $m_{1}+\left(m_{1}-m\right)$ após $N$ passos, sem barreira, chega em $m$ após $N$ passos com barreira. Portanto, a probabilidade total é dada por

$$
P\left(N, m, m_{1}\right)=P(N, m)+P\left(N, 2 m_{1}-m\right) .
$$

De fato, se considerarmos uma trajetória como OED que tenha sofrido exatamente uma reflexão em $m_{1}$, notamos que pela reflexão desta trajetória em torno da linha vertical que passa por $m_{1}$, obtemos uma trajetória levando ao ponto" imagem " $\left(2 m_{1}-m\right)$. Reciproca- 


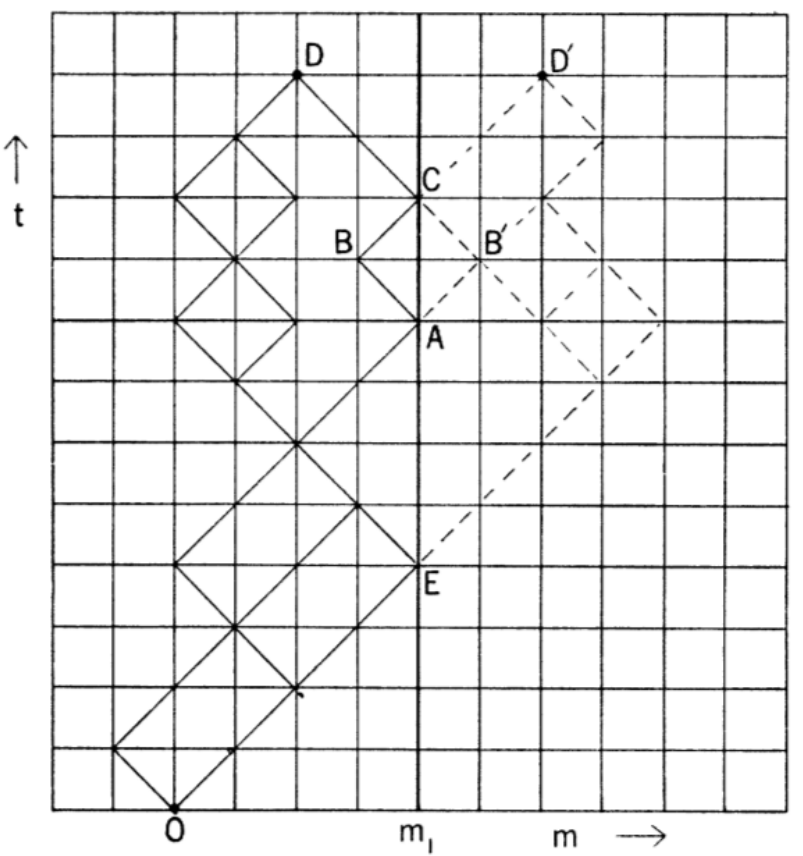

Figura 5.2: Passeio aleatório com barreira

mente, a cada trajetória que leva à imagem, tendo cruzado a linha através de $m_{1}$ uma vez, existe exatamente uma que leva à $m$ após uma reflexão. Assim, ao invés de contarmos duas vezes cada trajetória, refletida uma vez, basta considerar uma única trajetória levando à $\left(2 m_{1}-m\right)$.

Consideremos agora uma trajetória como OABCD, que leva à $m$ após duas reflexões. Uma trajetória deste tipo deve ser contada quatro vezes. Mas existem trajetórias, OAB'CD e OABCD', levando ao ponto "imagem “ e uma terceira OAB'CD que devem ser excluídas por conta da barreira. Estas três trajetórias adicionais juntas com OABCD dão exatamente quatro trajetórias levando à $m$ ou à sua "imagem" $2 m_{1}-m$ na ausência da barreira refletora. Assim, podemos usar os mesmo argumentos para generalizar a equação (5.24). Se tirarmos o limite para $N$ suficientemente grande, a equação (5.24) torna-se

$$
P\left(N, m, m_{1}\right)=\frac{2}{\sqrt{\pi N}}\left(\exp \left[-\frac{m^{2}}{2 N}\right]+\exp \left[-\frac{\left(2 m_{1}-m\right)^{2}}{2 N}\right]\right)
$$

Se usarmos o deslocamento $y=m l$ como sendo a variável e considerarmos a probabilidade $P\left(y, y_{1}, t\right) \Delta y$ que a partícula esteja no intervalo $[y, y+\Delta y]$, com $\Delta y>>l$, após um tempo $t$, durante o qual ele tomou $n t$ passos, na presença de uma barreira refletora em $y_{1}=m_{1} l$, temos que a probabilidade é dada como em (5.22), mas agora com $f\left(y, y_{1}, t\right)$ dada por 


$$
f\left(y, y_{1}, t\right)=\frac{1}{2 \sqrt{\pi D t}}\left(\exp \left[-\frac{y^{2}}{4 D t}\right]+\exp \left[-\frac{\left(2 y_{1}-y\right)^{2}}{4 D t}\right]\right)
$$

Note que

$$
\left[\frac{\partial f\left(y, y_{1}, t\right)}{\partial y}\right]_{y=y_{1}}=0
$$

\subsubsection{Barreira Absorvente em $m=m_{1}$}

Consideremos agora o caso no qual sempre que a partícula chegue em $m_{1}$, ela não sofra mais deslocamentos. Aqui surgem duas questões para este caso. A primeira é análoga ao caso anterior, ou seja, a probabilidade de que a partícula chegue em $m\left(m<<m_{1}\right)$ após $N$ passos. A segunda, característica do problema atual, é: Qual a taxa média com a qual a partícula irá depositar-se na "parede" absorvente?

Primeiramente pensemos na probabilidade $P\left(N, m, m_{1}\right)$. Notemos que ao contarmos o número de sequências distintas de passos que levam à $m$, devemos tomar cuidado ao excluirmos todas as sequências que incluem até mesmo uma única chegada à $m_{1}$. Dito de outra forma, se contarmos todas as possíveis sequências que levam à $m$, na ausência de uma "parede" absorvente, devemos então excluir um certo número de sequências "proibidas". Obviamente, por outro lado, todas tais sequências definem, de maneira única, uma outra sequência levando à imagem $\left(2 m_{1}-m\right)$ de $m$ com respeito a reta $m=m_{1}$, no plano $(N, m)$, como na figura (5.2), e reciprocamente. O que foi dito acima é que não devemos levar em consideração as "partículas" que cruzam, ou tocam, a reta $m=m_{1}$ em um tempo menor que $t$. Ou seja, devemos subtrair a solução "imagem", com respeito a reta $m=m_{1}$, da solução (5.22). Assim, a função densidade de probabilidade é dada por

$$
f\left(y, y_{1}, t\right)=\frac{1}{2 \sqrt{\pi D t}}\left(\exp \left[-\frac{y^{2}}{4 D t}\right]-\exp \left[-\frac{\left(2 y_{1}-y\right)^{2}}{4 D t}\right]\right) .
$$

Observe-se que

$$
\left.f\left(y, y_{1}, t\right)\right|_{y=y_{1}}=0 .
$$

Voltando a segunda questão, consistindo em saber qual a taxa com que a partícula se deposita na "barreira" absorvente. Suponhamos primeiro que não existe uma "barreira " absorvente. Então, a chegada da partícula em $m_{1}$ após $N$ passos implica que sua posição após $N-1$ passos deveria ser $m_{1}-1$ ou $m_{1}+1$. Contudo, toda trajetória que chega em 
$\left(N, m_{1}\right)$ originada em $\left(N-1, m_{1}+1\right)$ é proibida, na presença de uma "barreira " absorvente, pois ele cruza a barreira. Devemos ter cuidado, pois poderíamos pensar que toda trajetória partindo de $\left(N-1, m_{1}-1\right)$ é permitida. De fato, um certo número destas trajetórias irá tocar ou cruzar a reta $m=m_{1}$ em um passo(tempo) menor que seu último passo. O número de trajetórias chegando em $\left(N-1, m_{1}-1\right)$, mas tendo ao menos um contato ou cruzado, em um passo menor, a reta $m=m_{1}$ é igual ao das que chegam em $\left(N-1, m_{1}+1\right)$. O que acontece aqui é que, por reflexão em torno da reta $m=m_{1}$, podemos obter de uma única trajetória levando a $\left(N-1, m_{1}+1\right)$ uma outra, imagem desta, levando à $\left(N-1, m_{1}-1\right)$, que é proibida. Portanto, o número de maneiras permitidas de chegar em $m_{1}$ pela primeira vez após $N$ passos é igual a todas as possíveis maneiras de chegar a $m_{1}$ após $N$ passos, sem berreira absorvente, menos duas vezes o número de maneiras de chegar em $\left(N-1, m_{1}+1\right)$ novamente na ausência de uma barreira absorvente, ou seja,

$$
\begin{aligned}
& \frac{N !}{\left[\left(N-m_{1}\right) / 2\right] !\left[\left(N+m_{1}\right) / 2\right] !}- \\
& 2 \frac{(N-1) !}{\left[\left(N+m_{1}\right) / 2\right] !\left[\left(N-m_{1}-2\right) / 2\right] !}= \\
& \frac{m_{1}}{N} \frac{N !}{\left[\left(N-m_{1}\right) / 2\right] !\left[\left(N+m_{1}\right) / 2\right] !} .
\end{aligned}
$$

Como cada trajetória tem a probabilidade de $(1 / 2)^{N}$, a probabilidade total, para chegar pela primeira vez em $m_{1}$, é dada por

$$
\tilde{P}\left(N, m_{1}\right)=\frac{m_{1}}{N} P\left(N, m_{1}\right) .
$$

Usando o teorema (2) e escrevendo

$$
y_{1}=m_{1} l, N=n t, \mathrm{e} D=\frac{1}{2} n l^{2} .
$$

Então, a probabilidade, $P\left(y_{1}, t\right) \Delta t$, da partícula chegar em $y=m l$ no intervalo $[t, t+\Delta t]$, pela primeira vez, é dada por

$$
P\left(y_{1}, t\right) \Delta t=\frac{1}{2} f\left(y_{1}, t\right) n \Delta t
$$

onde

$$
f\left(y_{1}, t\right)=\frac{y_{1}}{n t}\left[\frac{1}{(\pi D t)^{1 / 2}} \exp \left(-\frac{y_{1}^{2}}{4 D t}\right)\right]
$$


Devemos notar que o fator $1 / 2$ na equação (5.31) é devido ao fato de que $m$ cresce por um fator de dois em cada passo, desde que ele é par ou ímpar, dependendo do valo de $N$.

De (5.31) e (5.32) segue

$$
P\left(y, y_{1}, t\right)=\frac{y_{1}}{t}\left[\frac{1}{2(\pi D t)^{1 / 2}} \exp \left(-\frac{y_{1}^{2}}{4 D t}\right)\right] .
$$

A equação (5.33) pode ser pensada como dando uma fração de um grande número de partículas inicialmente na origem, e que são depositadas na parede absorvente por unidade de tempo, no tempo $t$. Em outras palavras, ela nos dá o "fluxo", ou seja o número de trajetórias cruzando a barreira por unidade de tempo.

Notemos que a equação (5.33) satisfaz a relação

$$
P\left(y, y_{1}, t\right)=-D\left[\frac{\partial f}{\partial y}\right]_{y=y_{1}} .
$$

\subsection{Equação de Fokker- Planck}

No que segue suponhamos que estamos interessados em estudar a evolução de uma variável aleatória discreta. Suponhamos que estamos interessados em encontrar a probabilidade que um conjunto de pontos do espaço de fase, com ação I no "tempo " (passo) $\mathrm{n}$, sofra um incremento $\Delta I$ no intervalo de tempo $\Delta n$. Esta probabilidade é dada por $W_{t}(I, n, \Delta I, \Delta n)$, chamada de função densidade de probabilidade de transição.

Seja $P(I, n+\Delta n) d I$ a probabilidade de que um ponto do espaço de fase no tempo $n+\Delta n$, esteja no intervalo $\{I, I+\Delta I\}$. Tem-se que

$$
P(I, n+\Delta n)=\int_{\Delta I} P(I-\Delta I, n) W_{t}(I-\Delta I, n, \Delta I, \Delta n) d(\Delta I) .
$$

Suponhamos que $\Delta n \gg 1$ e $\Delta I \ll I$, ou seja, a variação de $P$ para um passo $\Delta I$ é pequena. Assim, expandindo o integrando de (5.35), em série de Taylor, até segunda ordem em $\Delta I$, temos

$$
\begin{aligned}
P(I-\Delta I, n) W_{t}(I-\Delta I, n, \Delta I, \Delta n) & =P(I, 0, n) W_{t}(I, n, 0, \Delta n) \\
& -\frac{\partial\left[P(I, 0, n) W_{t}(I, n, 0, \Delta n)\right]}{\partial I} \Delta I \\
& +\frac{1}{2} \frac{\partial^{2}\left[P(I, 0, n) W_{t}(I, n, 0, \Delta n)\right]}{\partial I^{2}}(\Delta I)^{2}
\end{aligned}
$$

Observemos que, como $W_{t}$ é uma função densidade de probabilidade, tem-se 


$$
\int W_{t}(I, n, \Delta I, \Delta n) d(\Delta I)=1
$$

Portanto, integrando o primeiro termo de (5.36), obtemos $P(I, 0, n)$. Passando $P(I, 0, n)$ para o lado esquerdo da equação 5.36 , dividindo por $\Delta n$ e fazendo o limite $\Delta n \longrightarrow 0$, obtemos a equação

$$
\frac{\partial P}{\partial n}=-\frac{\partial(B P)}{\partial I}+\frac{1}{2} \frac{\partial^{2}(D P)}{\partial I^{2}}
$$

onde

$$
B(I)=\frac{1}{\Delta n} \int \Delta I W_{t}(I, n, \Delta I, \Delta n) d(\Delta I)=\frac{\langle\Delta I\rangle}{\Delta n}
$$

e

$$
D(I)=\frac{1}{\Delta n} \int(\Delta I)^{2} W_{t}(I, n, \Delta I, \Delta n) d(\Delta I)=\frac{\left\langle(\Delta I)^{2}\right\rangle}{\Delta n} .
$$

A equação (5.38) é conhecida como equação de Fokker-Planck. As equações (5.39) e (5.40) são os coeficiente de fricção e coeficiente de difusão, respectivamente.

\subsubsection{Equação de Fokker-Planck Para Um Sistema Hamiltoniano}

Suponhamos que temos um sistema Hamiltoniano, com função Hamiltoniana $H=$ $H(I, \theta, t)$, neste caso, os coeficientes $B(I)$ e $D(I)$, obedecem a seguinte relação

\section{Teorema 3.}

$$
B(I)=\frac{1}{2} \frac{d D}{d I}
$$

Demonstração. De fato, se expandirmos $I$ em séries de Taylor, temos

$$
I(t+\Delta t)=I(t)+\dot{I} \Delta t+\frac{1}{2} \ddot{I}(\Delta I)^{2} .
$$

Como, por hipótese, temos um sistema Hamiltoniano, segue que

$$
\dot{I}=-\frac{\partial H}{\partial \theta}
$$

donde

$$
\ddot{I}=-\frac{\partial^{2} H}{\partial \theta^{2}} \dot{\theta}-\frac{\partial^{2} H}{\partial \theta \partial I} \dot{I}-\frac{\partial^{2} H}{\partial \theta \partial t} .
$$

Usando as equações de Hamilton obtemos 


$$
\ddot{I}=-\frac{\partial}{\partial \theta}\left(\frac{\partial H}{\partial I}\right)+\frac{\partial}{\partial I}\left(\frac{\partial H}{\partial \theta}\right)^{2}-\frac{\partial}{\partial \theta}\left(\frac{\partial H}{\partial t}\right) .
$$

Substituindo (5.43) em (5.42), temos

$$
\Delta I=-\frac{\Delta H}{\Delta \theta} \Delta t+\frac{1}{2}(\Delta I)^{2}\left[-\frac{\partial}{\partial \theta}\left(\frac{\partial H}{\partial I}\right)+\frac{\partial}{\partial I}\left(\frac{\partial H}{\partial \theta}\right)^{2}-\frac{\partial}{\partial \theta}\left(\frac{\partial H}{\partial t}\right)\right] .
$$

Supondo que $H$ é periódico em $\theta$ e fazendo a média sobre $\theta$, tem-se

$$
\langle\Delta I\rangle_{\theta}=\frac{1}{2}(\Delta I)^{2} \frac{\partial}{\partial I}\left\langle\left(\frac{\partial H}{\partial \theta}\right)^{2}\right\rangle_{\theta}
$$

Notemos que, até segunda ordem em $\Delta t$, podemos escrever

$$
\Delta I \Delta I=\dot{I}^{2}(\Delta t)^{2}=\left(\frac{\partial H}{\partial \theta}\right)^{2}(\Delta t)^{2} .
$$

Novamente, medianizando sobre $\theta$, obtemos

$$
\left\langle(\Delta I)^{2}\right\rangle_{\theta}=(\Delta t)^{2}\left\langle\left(\frac{\partial H}{\partial \theta}\right)^{2}\right\rangle_{\theta}
$$

Usando (5.39),(5.40), (5.45) e (5.47), obtemos

$$
B(I)=\frac{1}{2} \frac{d D}{d I}
$$

Usando a relação (5.41), podemos reescrever a equação (5.38) como

$$
\frac{\partial P}{\partial t}=\frac{\partial}{\partial I}\left(\frac{1}{2} D \frac{\partial P}{\partial I}\right)
$$

Devemos observar que para descrever, estatisticamente, o transporte em um dado sistema Hamiltoniano usando a equação de Fokker-Planck, devemos garantir que certas condições sejam satisfeitas, a saber:

1. Suponhamos que, o conjunto de trajetórias, cuja evolução queremos calcular, está em uma região caótica do espaço de fase ( Para definir a caoticidade da região calculamos o Número de Lyapunov).

2. A região caótica, no espaço das ações, deve ser simplesmente conexa. Em caso contrário, o problema se torna bastante complicado, pois teríamos que introduzir um grande número de barreiras refletoras. 
3. O movimento deve ser considerado como um Processo Markoviano, ou seja, deveríamos ter um rápido decaimento das correlações. Vale observar, que esta condição não é satisfeita no caso do caos estável no Sistema Solar. Em uma trajetória caótica estável, a função de autocorrelação da ação, correspondendo ao semieixo, decai em uma escala de tempo da ordem do tempo de Lyapunov(isto é o inverso do maior número de Lyapunov). Todavia, as funções de auto correlação de outras duas ações, correspondendo à excentricidade e à inclinação, tem variação quase-periódica para cem vezes o tempo de Lyapunov. Assim, a correlação da excentricidade não decai rapidamente, logo não pode ser modelada com um passeio aleatório, no espaço das excentricidades(ver, Varvoglis (2005)).

4. O "tempo" (passo) do passeio aleatório deve ser maior do que o tempo de autocorrelação, $\Delta n \gg n_{c}$, enquanto que um passo típico nas ações deve ser pequeno, ou seja, $\Delta I \ll I$. Com isto exclui-se da modelagem por passeio aleatório os movimentos rápidos através da variedade instável de uma trajetória periódica de baixa ordem.

5. A variância da função densidade de probabilidade do passeio aleatório deve ser finita, ou seja, $\sigma \neq \infty$.

\subsection{Cálculo do Coeficiente de Difusão}

Nos artigos existentes, os autores calculam o coeficiente de difusão de muitas formas diferentes. No que segue apresentaremos o cálculo do coeficiente seguindo (Lega et al. (2003)) e em seguida seguindo (Chirikov (1979)).

\subsubsection{Coeficiente Segundo Froeschlé}

Consideremos o Hamiltoniano usado por (Lega et al. (2003))

$$
H=\frac{I_{1}^{2}}{2}+\frac{I_{2}^{2}}{2}+I_{3}+\epsilon \frac{1}{\cos \left(\varphi_{1}\right)+\cos \left(\varphi_{2}\right)+\cos \left(\varphi_{3}\right)+4},
$$

onde $I_{1}, I_{2}, I_{3} \in \mathbb{R}$ e $\varphi_{1}, \varphi_{2}, \varphi_{3} \in \mathbb{T}$ e $\epsilon$ é um pequeno parâmetro. As superfícies ressonantes para o Hamiltoniano (5.49) são dadas por

$$
k_{1} I_{1}+k_{2} I_{2}+k_{3}=0 \quad \operatorname{com}\left(k_{1}, k_{2}, k_{3}\right) \in \mathbb{Z}^{3} \backslash \mathbf{0}
$$


No que segue consideraremos a particular linha ressonante, $I_{1}-2 I_{2}=0$, obtida numericamente (ver,Lega et al. (2003)). Verificou-se que os valores $I_{1}(t)-2 I_{2}(t)$ são constantes e próximos a zero, logo não pode haver difusão na direção transversal a linha ressonante. Por outro lado, verificou-se uma variação nos valores $2 I_{1}(t)+I_{2}(t)$, isto faz com que exista uma difusão lenta na direção ao longo da linha ressonante.

\subsubsection{Coeficiente de Difusão}

Vale notar que não existe um modelo analítico para a difusão lenta, portanto para calcular o coeficiente de difusão assumimos que o fenômeno se comporta como movimento Browniano. Sejam, $I_{1, j}(0)$ e $I_{2, j}(0)$, para $j=1, \ldots, N$ condições iniciais para um conjunto de $N$ órbitas, com $I_{1, j}(t)$ e $I_{2, j}$ os correspondentes valores no tempo $t$, definamos

$$
S(t)=\frac{1}{N} \sum_{j=1}^{N}\left[\left(I_{2, j}(t)+2 I_{1, j}(t)\right)-\left(I_{2, j}(0)+2 I_{1, j}(0)\right)\right]^{2} .
$$

É importante notar que os termos da soma (5.51) são quadrados das distâncias das ações entre os valores projetados na linha ressonante $I_{1}=2 I_{2}$ e os valores inciais. Seja $T$ uma fração do tempo total de integração, para qualquer que seja $n \in \mathbb{N}$, consideremos

$$
\bar{S}(n T)=\frac{1}{M_{n}} \sum_{j:\left|\varphi_{1, j}(t)\right|+\left|\varphi_{2, j}(t)\right| \leq 0.05, \varphi_{3, j}(t)=0}\left[\left(I_{2, j}(t)+2 I_{1, j}(t)\right)-\left(I_{2, j}(0)+2 I_{1, j}(0)\right)\right]^{2},
$$

onde $M_{n}$ é o número de pontos da seção $S$, definida por

$$
\begin{aligned}
\left|\varphi_{1, j}(t)\right|+\left|\varphi_{2, j}(t)\right| & \leq 0.05 \\
\varphi_{3, j}(t) & =0
\end{aligned}
$$

para $t$ no intervalo $(n-1) T \leq t \leq n T$. Observemos figura(5.3) que $\bar{S}$ cresce linearmente com o tempo, definimos o coeficiente de difusão $D$ como sendo a inclinação da regressão linear. Note que a inclinação das duas funções (5.51) e (5.52) é a mesma, ou seja, temos o mesmo valor de $D$, para ambas.

\subsection{Taxa de Difusão Segundo Chirikov}

Segundo Chirikov (ver,Chirikov (1979)) a taxa de Difusão é dada por

$$
D_{k}=\frac{2}{N_{k}\left(N_{k}-1\right)} \sum_{m>n} \frac{\left(\bar{H}_{m}-\bar{H}_{n}\right)^{2}}{(\Delta t)_{k}(m-n)} .
$$




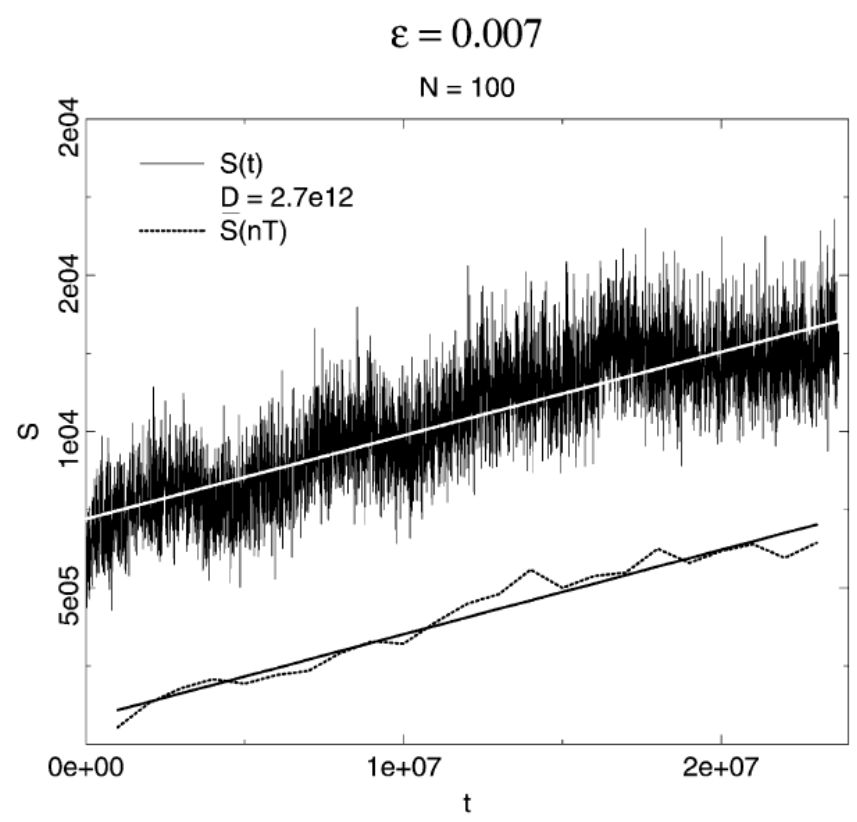

Figura 5.3: Evolução de $S(t)$ e $\bar{S}(n T)$ para 100 órbitas com $\epsilon=0.007$. O declive é o mesmo para as duas funções e nos dá o coeficiente de difusão $D=2.7 \times 10^{-12}$.

Neste caso para calcularmos o coeficiente subdividimos o tempo total do movimento $t_{\text {máx }}$ em subintervalos $N_{k}$ de comprimento $(\Delta t)_{k}$. Medianizamos o valor da energia $H(t)$ em cada um destes intervalos obtendo $\bar{H}_{m}$. Com isto, a taxa de difusão para um dado par $\bar{H}_{m}, \bar{H}_{n}$, separados pelo intervalo $(\Delta t)_{k}(m-n)$ é dada por

$$
\frac{\left(\bar{H}_{m}-\bar{H}_{n}\right)^{2}}{(\Delta t)_{k}(m-n)}
$$

Em seguida medianizamos esta taxa sobre todas as combinações com $m \neq n$ obtendo a equação (5.53). Vale notar que não foi possível encontrarmos uma dedução e maiores referência em relação à fórmula (5.53). Além disso, as referências onde se encontra esta fórmula não nos fornecem detalhes a respeito da obtenção da mesma. 
Capítulo 6

\section{Teoria da difusão de Chirikov}

Neste capítulo apresentamos o desenvolvimento de Chirikov para estudar a dinâmica de sitemas multidimensionais com ressonâncias que será aplicado ao Hamiltoniano do sistema GJ581, para maiores detalhes do desenvolvimento de Chirikov ver, Chirikov (1979), Cachucho da Silva, F. (2003) e Cachucho et al. (2010).

Consideremos um sistema Hamiltoniano com várias perturbações periódicas que podem causar ressonância. As condições iniciais são escolhidas de modo que o sistema esteja no domínio de uma ressonância essencial, chamada de ressonância guia. O termo da perturbação correspondente a ressonância guia é separado dos demais, chamadas ressonâncias perturbadoras, tal que o Hamiltoniano possui a seguinte forma

$$
H=H_{0}(\mathbf{I})+\epsilon V_{G}(\mathbf{I}) \cos \left(\mathbf{m}_{\mathbf{G}} \cdot \boldsymbol{\theta}\right)+\epsilon V(\mathbf{I}, \boldsymbol{\theta})
$$

com

$$
\epsilon V=\epsilon \sum_{\mathbf{m} \neq \mathbf{m}_{\mathrm{G}}} V_{\mathbf{m}} \cos (\mathbf{m} \cdot \boldsymbol{\theta})
$$

Aqui , $V_{G}$ e $\mathbf{m}_{G}$ representam a amplitude e o vetor da ressonância guia, respectivamente, enquanto $V$ e $\mathbf{m}$ são a amplitude e o vetor das ressonâncias perturbadoras, respectivamente. Lembrando que $(\mathbf{I}, \boldsymbol{\theta})$ são as variáveis ação-ângulo usuais de $N$ dimensões do Hamiltoniano não perturbado $H_{0}$ para $N \geq 3$.

É importante notar que os vetores $\mathbf{m}, \mathbf{m}_{\mathbf{G}}$ pertencem a $\mathbb{Z}^{n} /\{\mathbf{0}\}$ e $V_{G}, V_{\mathbf{m}}$ são funções reais das ações I. O parâmetro perturbador $\epsilon \in \mathbb{R}$ é tal que $\epsilon<<1$.

A condição de ressonância é fixada pela equação

$$
S\left(\mathbf{I}^{r}\right)=\mathbf{m}_{\mathbf{G}} \cdot \omega\left(\mathbf{I}^{r}\right)=0
$$


A superfície $S\left(\mathbf{I}^{r}\right)=0$, no espaço das ações, é dita superfície ressonante.

\subsection{Geometria da ressonância guia no espaço das ações}

Inicialmente, suponhamos que $V_{\mathbf{m}}=0$ para $\mathbf{m} \neq \mathbf{m}_{G}$, ou seja, sem perturbação e escolhamos condições iniciais próximas a separatriz da ressonância guia.

Note que no $\boldsymbol{\omega}$-espaço, podemos melhor visualizar a condição de ressonância

$$
\mathbf{m}_{\mathbf{G}} \cdot \omega\left(\mathbf{I}^{r}\right)=0 .
$$

De fato, neste espaço ela representa exatamente um plano de dimensão $(N-1)$, cuja normal é o vetor ressonante $\mathbf{m}_{G}$. No I-espaço, ela nos leva a uma superfície $S\left(\mathbf{I}^{r}\right)=0$ de dimensão $(N-1)$, cuja normal local no ponto $\mathbf{I}=\mathbf{I}^{r}$ é dada pelo vetor

$$
\mathbf{n}^{r}=\left(\frac{\partial}{\partial \mathbf{I}}\left[\mathbf{m}_{G} \cdot \omega(\mathbf{I})\right]\right)_{\mathbf{I}=\mathbf{I}^{r}}
$$

De fato, sabemos que o gradiente de uma função num ponto é perpendicular a superfície de nível da função que passa por este ponto.

Note-se que, considerando a superfície $H_{0}(\mathbf{I})=E$ de dimensão $(N-1)$, no I-espaço, e supondo que $\boldsymbol{\omega}\left(\boldsymbol{I}^{r}\right)$ seja bijetiva, isto é, $\operatorname{det}\left(\frac{\partial H_{0}}{\partial \mathbf{I}^{r}}\right) \neq 0$, podemos escrever

$$
\tilde{H}_{0}(\boldsymbol{\omega})=H_{0}(\mathbf{I}(\boldsymbol{\omega})=E,
$$

agora no $\boldsymbol{\omega}$ - espaço.

Deve-se notar também que o subespaço definido pela interseção da superfície ressonante $S\left(\mathbf{I}^{r}\right)$ com a superfície de energia constante possui $(N-2)$-dimensões.

Observe-se que por definição $\boldsymbol{\omega}(\mathbf{I})=\frac{\partial H_{0}}{\partial \mathbf{I}}, \operatorname{logo} \boldsymbol{\omega}$ é normal a superfície de energia no I-espaço. Essa condição junto com a condição de ressonância (6.3), nos diz que o vetor ressonante pertence ao plano tangente à superfície no ponto $\mathbf{I}=\mathbf{I}^{r}$. Além disso, $\dot{\mathbf{I}}$ é paralelo ao vetor ressonante $\mathbf{m}_{G}$. De fato,

$$
\dot{\mathbf{I}}=\frac{\partial H}{\partial \theta}=\frac{\partial H_{0}}{\partial \theta}-\epsilon V_{G}(\mathbf{I}) \sin \left(\mathbf{m}_{G} \cdot \theta\right) \cdot \mathbf{m}_{G}=-\epsilon V_{G}(\mathbf{I}) \sin \left(\mathbf{m}_{G} \cdot \theta\right) \cdot \mathbf{m}_{G} .
$$

Devemos lembrar que estamos considerando apenas $H_{0}$ e o termo ressonante. Fisicamente, isto significa que, que neste espaço, o movimento sob uma simples perturbação ressonante, ocorre sob o plano tangente à superfície de energia na direção do vetor ressonante $\mathbf{m}_{G}$. 
Portanto podemos afirmar que, numa primeira aproximação, $\epsilon \rightarrow 0$, a perturbação ressonante preserva a energia não perturbada $H_{0}$.

\subsection{Mudança de base local}

Agora vamos introduzir uma transformação canônica $(\mathbf{I}, \theta) \longrightarrow(\mathbf{p}, \psi)$, definida pela função geratriz

$$
F(\mathbf{p}, \boldsymbol{\theta})=\sum_{i=1}^{N}\left(I_{i}^{r}+\sum_{k=1}^{N} p_{k} \mu_{k i}\right) \theta_{i},
$$

onde $\left(\boldsymbol{\mu}_{k i}\right)$ é uma matriz $N \times N$ inversível com $\mu_{1 i}=\left(\mathbf{m}_{G}\right)_{i}$.

Temos que as equações desta transformação são dadas por

$$
I_{i}=\frac{\partial F}{\partial \theta_{i}}=I_{i}^{r}+\sum_{k=1}^{N} p_{k} \mu_{k i} ; \quad \psi_{k}=\frac{\partial F}{\partial p_{k}}=\sum_{l=1}^{N} \mu_{k l} \theta_{l}
$$

Observemos que estamos supondo as fases $\psi_{k}, k=1, \ldots, N$ não-degeneradas, ou seja, $\frac{\partial H_{0}}{\partial \mathbf{I}_{k}} \neq 0$. Podemos pensar nesta transformação, como uma mudança de base local ao invés de uma mudança de coordenadas local. De fato, note que, o vetor ação cujo as componentes são $\left(\mathbf{I}_{j}-\mathbf{I}_{j}^{r}\right)$ na base original, digamos, $\left\{\mathbf{u}_{j}, j=1, \ldots, N\right\}$, tem componentes $p_{j}$ na nova base $\left\{\boldsymbol{\mu}_{j}, j=1, \ldots, N\right\}$, construída com base na geometria das ressonâncias no espaço das ações, como segue.

Seja, $\boldsymbol{\mu}_{1 i}=\mathbf{m}_{1} \equiv \mathbf{m}_{G}$, isto é, a primeira linha da matriz $\left(\mu_{k i}\right)$ é o vetor ressonante $\mathbf{m}_{G}$.

Note que, com essa escolha, a fase ressonante é

$$
\psi_{1}=\sum_{l=1}^{N} \mu_{1 l} \theta_{l}=\mathbf{m}_{G} \cdot \boldsymbol{\theta} .
$$

Observe que $\boldsymbol{\mu}_{1 i}$ é ortogonal à $\omega^{r}$, pois $\mathbf{m}_{G} \perp \boldsymbol{\omega}^{r}$. Portanto, o vetor $\boldsymbol{\mu}_{2 i}=\frac{\omega^{r}}{\left\|\boldsymbol{\omega}^{r}\right\|}$ é ortogonal à $\boldsymbol{\mu}_{1 i}$.

Os outros vetores são $\boldsymbol{\mu}_{k i}=\mathbf{e}_{k}, k=3, \ldots, N$, tais que os $\mathbf{e}_{k}$ são ortonormais entre si, ortogonais a $\mu_{2}$ e a $\mathbf{n}^{r}$.

Geometricamente, para $N=3$, temos que: o vetor ressonante $\mathbf{m}_{G}$ pertence ao plano tangente à superfície de energia no ponto ressonante $\mathbf{I}^{r}$, enquanto que o vetor frequência $\boldsymbol{\omega}^{r}$ é normal a este plano. 
É importante notar que, todos os vetores $\mathbf{e}_{k}$ são ortogonais a $\boldsymbol{\mu}_{1 i}=\mathbf{m}_{G}$, exceto um deles, $\operatorname{digamos} \mathbf{e}_{s}$.

De fato, seja

$$
\mathbf{e}_{s} \in \prod_{H_{0}=c t e} \cap \prod_{S^{r}=c t e}
$$

onde $\prod_{H_{0}}$ e $\prod_{S^{r}}$ são os planos tangentes as superfícies de energia constante $H_{0}$ e ressonante $S^{r}$, respectivamente.

Observe que sendo $\mathbf{e}_{s} \perp \mathbf{n}^{r}$, podemos dizer que $\mathbf{e}_{s}=\alpha \cdot\left(\mathbf{n}^{r} \times \omega^{r}\right), \alpha \in \mathbb{R}$.

Suponha agora que $\mathbf{e}_{s} \cdot \mathbf{m}_{G}=0$, então $\alpha \cdot\left(\mathbf{n}^{r} \times \omega^{r}\right) \cdot \mathbf{m}_{G}=0$, o que é uma contradição, visto que $\mathbf{n}^{r}, \omega^{r}$ e $\mathbf{m}_{G}$, não são coplanares em geral.

Vale notar que, no caso particular $\boldsymbol{\omega}(\mathbf{I})=\mathbf{I}$, ou seja, $H=\mathbf{I} / 2$, temos $\mathbf{n}^{r}$ e $\mathbf{m}_{G}$ com mesma direção, e portanto, neste caso, $\mathbf{e}_{s} \perp \mathbf{m}_{G}$.

Note-se que, sendo $\mathbf{p}=p_{k} \boldsymbol{\mu}_{k i}, k=1, \ldots, 3$, podemos dizer que $p_{1}$ mede a variação do movimento do ponto ressonante através da superfície da ressonância guia; $p_{2}$ mede a variação da energia não perturbada $H_{0}$ e $p_{3}$ mede a variação do valor ressonante ao longo da superfície da ressonância guia.

No caso de uma perturbação apropriada, $|\epsilon|<<1$, as variações de $p_{1}$ e $p_{2}$ são limitadas, e neste caso a difusão não é esperada. Observe que $p_{3}$ está na direção onde a difusão de Arnold provavelmente deveria ocorrer.

Portanto, para o caso de um sistema Hamiltoniano tridimensional autônomo, a difusão ocorre sobre uma curva, interseção da superfície ressonante de dimensão 2 com a superfície de energia constante de dimensão 2. Para $N \geq 3$ graus de liberdade, este subespaço interseção das duas superfícies de dimensão $(N-1)$ possui $(N-2)$ dimensões. Em (Chirikov (1979)), Chirikov chama esse subespaço de variedade de difusão.

Os $N-2$ vetores $\mathbf{e}_{k}$ estão localizados sob um plano tangente à variedade de difusão chamado plano de difusão. Assim, na nova base o vetor ação pode ser escrito como

$$
\mathbf{p}=p_{1} \mathbf{m}_{G}+p_{2} \frac{\omega^{r}}{\left\|\omega^{r}\right\|}+\mathbf{q}
$$

onde $\mathbf{q}=\sum_{k=3}^{N} q_{k} \mathbf{e}_{k} \operatorname{com} q_{k}=p_{k}$. 


\subsection{Hamiltoniano Nas Novas Ações}

Agora vamos escrever o Hamiltoniano(6.1) em termos das novas componentes da ação p, ou seja, nas novas variáveis.

Expandindo $H_{0}$ em série de Taylor ${ }^{1}$, até segunda ordem em $p_{k}$, temos

$$
\begin{aligned}
H_{0}(\mathbf{I}) & \approx H_{0}\left(\mathbf{I}^{\mathbf{r}}\right)+\frac{\partial H_{0}}{\partial \mathbf{I}}\left(\mathbf{I}^{\mathbf{r}}\right)\left(\mathbf{I}-\mathbf{I}^{\mathbf{r}}\right)+\frac{1}{2} \frac{\partial^{2} H_{0}}{\partial I_{i} \partial I_{j}}\left(\mathbf{I}^{\mathbf{r}}\right)\left(\mathbf{I}-\mathbf{I}^{\mathbf{r}}\right)^{2} \\
& =H_{0}\left(\mathbf{I}^{\mathbf{r}}\right)+\boldsymbol{\omega}^{r}\left(\mathbf{I}-\mathbf{I}^{\mathbf{r}}\right)+\frac{1}{2}\left(\mathbf{I}-\mathbf{I}^{\mathbf{r}}\right) \frac{\partial \boldsymbol{\omega}^{r}}{\partial I_{j}}\left(\mathbf{I}-\mathbf{I}^{\mathbf{r}}\right)
\end{aligned}
$$

de onde obtemos

$$
\begin{aligned}
H_{0}(\mathbf{I}) & \simeq H_{0}\left(\mathbf{I}^{r}\right)+\sum_{i=1}^{N} \sum_{k=1}^{N} \omega_{i}^{r} \mu_{i k} p_{k} \\
& +\frac{1}{2} \sum_{i=1}^{N} \sum_{j=1}^{N} \sum_{k=1}^{N} \sum_{l=1}^{N}\left(\frac{\partial \omega_{i}}{\partial I_{j}}\right)_{\mathbf{I}=\mathbf{I}^{\mathbf{r}}} \mu_{l j} \mu_{i k} p_{k} p_{l} .
\end{aligned}
$$

Lembrando que a nova fase $\psi$ ressonante é dada por $\psi_{1}=\sum_{l} \mu_{1 l} \theta_{l}=\mathbf{m}_{G} \cdot \boldsymbol{\theta}$, temos que

$$
\epsilon V_{G}(\mathbf{I}) \cos \left(\mathbf{m}_{\mathbf{g}} \cdot \boldsymbol{\theta}\right)=\epsilon V_{G}(\mathbf{p}) \cos \left(\psi_{1}\right)
$$

Além disso,

$$
\epsilon \sum_{\mathbf{m} \neq \mathbf{m}_{\mathrm{G}}} V_{\mathbf{m}}\left(\mathbf{I}^{r}\right) \cos (\mathbf{m} \cdot \boldsymbol{\theta})=\epsilon \sum_{\mathbf{m} \neq \mathbf{m}_{\mathrm{G}}} V_{\mathbf{m}}(\mathbf{p}, \boldsymbol{\psi}) \cos \left(\boldsymbol{\nu}_{m} \cdot \boldsymbol{\psi}\right)
$$

notemos que $\boldsymbol{\nu}_{m}$ é o vetor ressonante na nova base.

Assim, o Hamiltoniano (6.1) é dado por

$$
H(\mathbf{p}, \boldsymbol{\psi}) \approx H_{0}\left(\mathbf{I}^{r}\right)+\sum_{i=1}^{N} \sum_{k=1}^{N} \omega_{i}^{r} \mu_{i k} p_{k}+\sum_{k=1}^{N} \sum_{l=1}^{N} \frac{p_{k} p_{l}}{2 M_{k l}}+\epsilon V_{G}(\mathbf{p}) \cos \left(\psi_{1}\right)+\epsilon V\left(\varphi_{\mathbf{m}}\right)
$$

onde

\footnotetext{
${ }^{1}$ Lembremos que $\frac{\partial^{2} f}{\partial x_{i} \partial x_{j}}(a) \mathbf{v}^{2}=\sum_{i} \sum_{j} \frac{\partial^{2} f}{\partial x_{i} \partial x_{j}}(a) \alpha_{i} \alpha_{j}$, onde $\mathbf{v}=\left(\alpha_{1}, \ldots, \alpha_{n}\right)$
} 


$$
\varphi_{m}=\nu_{m} \cdot \boldsymbol{\psi}
$$

$\mathrm{e}$

$$
\frac{1}{M_{k l}}=\sum_{i=1}^{N} \sum_{j=1}^{N} \mu_{k i}\left(\frac{\partial \omega_{i}}{\partial I_{j}}\right)_{\mathbf{I}=\mathbf{I}^{\mathbf{r}}} \mu_{l j}
$$

Observe que $\sum_{i=1}^{N} \omega^{r} \mu_{k i}=0$ para $k \neq 2$, pelas condições de ortogonalidades, e no caso $k=2$ temos

$$
\sum_{i=1}^{N} \omega^{r} \mu_{2 i}=\boldsymbol{\omega}^{r} \cdot \frac{\boldsymbol{\omega}^{r}}{\left\|\boldsymbol{\omega}^{r}\right\|}=\left\|\boldsymbol{\omega}^{r}\right\| .
$$

Agora, desprezando o termo constante do Hamiltoniano (6.15), usando o observado acima para simplificarmos o termo linear, separando do termo quadrático o caso $l=k=1$ teremos

$$
H(\mathbf{p}, \boldsymbol{\psi}) \approx \frac{p_{1}^{2}}{2 M_{G}}+\epsilon V_{G}(\mathbf{p}) \cos \psi_{1}+\|\omega\| p_{2}+\sum_{k=1}^{N} \sum_{l=1}^{N} \frac{p_{k} p_{l}}{2 M_{k l}}+\epsilon V(\mathbf{p}, \boldsymbol{\psi}),
$$

onde

$$
\frac{1}{M_{G}}=\frac{1}{M_{11}}=m_{G i} \frac{\partial \omega_{i}^{r}}{\partial I_{j}} m_{G j}
$$

Notemos que na ausência de perturbação, ou seja, quando

$$
\epsilon V(\mathbf{p}, \boldsymbol{\psi}) \rightarrow 0
$$

o novo Hamiltoniano não depende das variáveis $\psi_{2}, \ldots, \psi_{N}, \operatorname{logo}$ os $p_{2}, \ldots, p_{N}$ são constantes, isto é, são integrais do movimento, e sem perda de generalidade podemos tomá-las iguais a zero para que $\mathbf{I}^{r}$ seja um ponto da órbita. Portanto o Hamiltoniano (6.18) reduz-se a

$$
H(\mathbf{p}, \boldsymbol{\psi}) \approx H_{1}\left(p_{1}, \psi_{1}\right)+\epsilon V(\boldsymbol{\psi})
$$

onde

$$
H_{1}\left(p_{1}, \psi_{1}\right)=\frac{p_{1}^{2}}{2 M_{G}}+\epsilon V_{G}(\mathbf{p}) \cos \psi_{1}
$$

o Hamiltoniano (6.22) é ressonante associado a ressonância guia, é o Hamiltoniano associado ao pêndulo simples. Note que o ponto de equilíbrio estável do pêndulo é $\psi_{1}=\pi$ se $M_{G} V_{G}>0$, ou $\psi_{1}=0$ se $M_{G} V_{G}<0$. 


\subsection{Perturbação}

Como foi dito acima, para $V=0$ os $p_{k}$ são integrais do movimento, então $H_{1}$ também é uma integral. Portanto, temos um conjunto completo de $N$ integrais não perturbadas: $H_{1}, p_{2}, p_{k}, k=3, \ldots, N$. Todavia, se adicionarmos a perturbação estas quantidades variam com o tempo. De fato, as equações de movimento para o Hamiltoniano (6.18) nos dará

$$
\dot{\psi}_{j}=\frac{\partial H}{\partial p_{j}}, \quad j=1, \ldots, N
$$

Note-se que derivando (6.21) em relação a $p_{1}$ tem-se

$$
\dot{\psi}_{1}=\frac{p_{1}}{M_{G}}+\sum_{l=2}^{N} \frac{p_{l}}{M_{1 l}}
$$

donde

$$
p_{1}=M_{G} \dot{\psi}_{1}-\sum_{l=2}^{N} \frac{M_{G} p_{l}}{M_{1 l}} .
$$

Agora, derivando (6.18) para $k>1$ e usando o fato de que para $V=0, p_{l}(l \neq 2)$ são constantes, obtemos

$$
\dot{\psi}_{k}=\left\|\omega^{r}\right\| \delta_{k 2}+\sum_{l=1}^{N} \frac{p_{l}}{M_{k l}}
$$

$\operatorname{com} \delta_{k 2}=0$ pra $k \neq 2$ e $\delta_{22}=1$.

Observe que, usando (6.25), podemos escrever a equação (6.26) como

$$
\dot{\psi}_{k}=\left\|\omega^{r}\right\| \delta_{k 2}+\frac{M_{G} \dot{\psi}_{1}}{M_{k 1}}+\sum_{l=2}^{N}\left(\frac{1}{M_{k l}}-\frac{M_{G}}{M_{k 1} M_{1 l}}\right) p_{l}
$$

Assim, integrando a equação (6.27) teremos

$$
\psi_{k}=\left\|\omega^{r}\right\| \delta_{k 2} t+\sum_{l=2}^{N}\left(\frac{1}{M_{k l}}-\frac{M_{G}}{M_{k 1} M_{1 l}}\right) p_{l} t+\frac{M_{G}}{M_{k 1}} \psi_{1}(t)+\psi_{k 0}, \quad k>1
$$

onde $\psi_{k 0}$ são constantes de integração.

Para obtermos $\varphi_{\mathbf{m}}(t)$, temos que calcular o produto $\sum_{i} \nu_{i} \psi_{i}$. Note-se que, utilizando (6.28), este produto tem a seguinte forma

$$
\nu_{k} \cdot \psi_{k}=\nu_{k}(\mathbf{m}) \frac{M_{G}}{M_{k 1}} \psi_{1}+\sum_{l=2}^{N} \nu_{k}\left(\frac{1}{M_{k l}}-\frac{M_{G}}{M_{k 1} M_{1 l}}\right) p_{l} t+\nu_{k}\left\|\omega^{r}\right\| \delta_{k 2} t+c t e .
$$


Logo podemos reescrever

$$
\varphi_{\mathbf{m}}=\mathbf{m} \cdot \boldsymbol{\theta}=\boldsymbol{\nu} \cdot \boldsymbol{\psi}=\xi_{\mathbf{m}} \psi_{1}(t)+\omega_{\mathbf{m}} t+\beta_{\mathbf{m}}+K_{\mathbf{m}}
$$

com

$$
\begin{aligned}
\xi_{\mathbf{m}} & =\sum_{k}^{N} \nu_{k}(\mathbf{m}) \frac{M_{G}}{M_{k 1}} \\
\omega_{\mathbf{m}} & =\mathbf{m} \cdot \boldsymbol{\omega}^{r} \\
K_{\mathbf{m}} & =\sum_{l=2}^{N} \nu_{k}(\mathbf{m})\left(\frac{1}{M_{k l}}-\frac{M_{G}}{M_{k 1} M_{1 l}}\right) p_{l} t
\end{aligned}
$$

$\beta_{\mathbf{m}}$ é constante.

Note-se também que o segundo termo de (6.31) é obtido levando-se em conta que

$$
\omega_{\mathbf{m}}=\mathbf{m} \cdot \boldsymbol{\omega}^{r}=\left(\sum_{i} \nu_{i} \mu_{i}\right) \cdot\left(\boldsymbol{\mu}_{2}\left\|\boldsymbol{\omega}^{r}\right\|\right)
$$

e o fato de que, como $\boldsymbol{\mu}_{2} \perp \boldsymbol{\mu}_{i}, \forall i \neq 2$. Então,

$$
\omega_{\mathbf{m}}=\mathbf{m} \cdot \boldsymbol{\omega}^{r}=\nu_{2}(\mathbf{m})\left\|\boldsymbol{\omega}^{r}\right\|
$$

Vamos calcular a variação, no tempo, da integral não perturbada. Utilizando o Hamiltoniano (6.21) note que, $\dot{p}_{k}=-\partial H / \partial \psi_{k}, k \neq 1$, obtem-se

$$
\dot{p}_{k} \approx \epsilon \sum_{\mathbf{m} \neq \mathbf{m}_{G}} \nu_{k}(\mathbf{m}) V_{\mathbf{m}}^{r} \sin \varphi_{\mathbf{m}}(t)
$$

onde $V_{\mathbf{m}}^{r}=V_{\mathbf{m}}\left(\mathbf{I}^{r}\right)$.

É importante observar que a equação (6.34) é verdadeira para todas as componentes do momento $\mathbf{p}$, exceto $p_{1}$. Como $p_{1}$ não é uma integral, Chirikov usa $H_{1}$ com a intenção de construir um wisker map para descrever a difusão de Arnold. Contudo, neste trabalho para o estudo de ressonâncias de três corpos, calcularemos a evolução das componentes do momento p usando o mapa Simplético de Hadjidemetriou.

\subsection{Estimativa da Variação Total das Componentes do momento p}

Aqui usamos a construção da variação de Chirikov para obtermos uma estimativa teórica para a difusão lenta, e então calculamos a variação total dos $p_{k}$. 


\subsubsection{Equação da Separatriz}

Se $\epsilon$ é suficientemente pequeno, os domínios do espaço de fase associados a todas as ressonâncias presentes em (6.2) não tem overlap. Então existe um procedimento conhecido para mudarmos $\psi_{1}$ e $\dot{\psi}_{1}$ pelos valores sobre a separatriz não perturbada, para isto resolvemos analiticamente a equação (6.34).

Primeiro integramos (6.34) sobre uma trajetória completa interior à camada estocástica assumindo que $\psi_{1}=\psi_{1}^{\text {sx }}$ e $K_{\mathbf{m}}=0$. De fato, como mencionado antes, para $V=0$ os $p_{l}, l \neq 1$ são integrais do movimento e as fases $\varphi_{\mathbf{m}}$ podem ser estimadas considerando estes $p_{l}=0$, tal que $K_{\mathbf{m}}=0$. Então, a variação total dos $p_{k}^{\prime} s$ são dadas por

$$
\triangle p_{k} \approx \sum_{\mathbf{m} \neq \mathbf{m}_{\mathrm{G}}} \nu_{k}(\mathbf{m}) V_{\mathbf{m}}^{r} \int_{-\infty}^{+\infty} \sin \varphi_{\mathbf{m}}^{s x}(t) d t,
$$

onde $\varphi_{\mathbf{m}}^{s x}(t)=\xi_{\mathbf{m}} \psi_{1}^{s x}(t)+\omega_{\mathbf{m}} t+\beta_{\mathbf{m}}$.

Da formulação de Chirikov (ver, Chirikov (1979)), ( para maiores detalhes ver, Ferraz-Mello (2007))), temos que a separatriz não perturbada é definida por

$$
\begin{aligned}
& \psi_{1}^{s x}(t)=4 \arctan \left(e^{ \pm \Omega_{G}\left(t-t^{0}\right)}\right) \\
& p_{1}^{s x}= \pm 2\left|M_{G}\right| \Omega_{G} \sin \left(\frac{\psi_{1}^{s x}}{2}\right)
\end{aligned}
$$

onde

$$
\Omega=\sqrt{\epsilon\left|\frac{V_{G}}{M_{G}}\right|}
$$

é a frequência própria do Hamiltoniano do pêndulo $H_{1}$. O sinal duplo corresponde aos dois ramos da separatriz: $\mathrm{O}$ sinal positivo corresponde à separatriz superior $\left(0 \leq \psi_{1}^{s x}<2 \pi\right)$, enquanto que o negativo $\left(-2 \pi \leq \psi_{1}^{s x}<0\right)$ corresponde à separatriz inferior. Os pontos de equilíbrio estáveis estão em $\psi_{1}= \pm \pi$, respectivamente.

Introduzimos a variável $\tau=\Omega_{G}\left(t-t^{0}\right)$, com $\psi_{1}^{s x}\left(t^{0}\right)=\psi_{1}^{0}= \pm \pi$. Então, para $\psi_{1}^{s x}>$ 0 (separatriz inferior), temos

$$
\sin \varphi_{\mathbf{m}}^{s x}(t)=\sin \left(\xi_{\mathbf{m}} \psi_{1}^{s x}(\tau)+\lambda_{\mathbf{m}} \tau+\varphi_{\mathbf{m}}^{0}\right),
$$

onde $\varphi_{\mathbf{m}}^{0}=\xi_{\mathbf{m}} \psi_{1}^{0}+\omega_{\mathbf{m}} t^{0}+\beta_{\mathbf{m}}$, como $\psi_{1}^{0}=\pi$, e 


$$
\lambda_{\mathbf{m}}=\frac{\omega_{\mathbf{m}}}{\Omega_{G}} .
$$

Note que, usando identidade do seno da soma, podemos reescrever (6.39) como

$$
\sin \varphi_{\mathbf{m}}^{s x}(t)=\sin \left(\xi_{\mathbf{m}} \psi_{1}^{s x}(\tau)+\lambda_{\mathbf{m}} \tau\right) \cos \varphi_{\mathbf{m}}^{0}+\cos \left(\xi_{\mathbf{m}} \psi_{1}^{s x}(\tau)+\lambda_{\mathbf{m}} \tau\right) \sin \varphi_{\mathbf{m}}^{0}
$$

Observe que substituindo (6.41) em (6.35), o primeiro termo não dará contribuição, pois por simetria

$$
\int_{-\infty}^{+\infty} \sin \left[\left(\xi_{\mathbf{m}} \psi_{1}^{s x}(\tau)+\lambda_{\mathbf{m}} \tau\right] d \tau=0\right.
$$

Quanto ao segundo termo de (6.41), sua contribuição é determinada pelos sinais relativos de $\xi_{\mathbf{m}}$ e $\lambda_{\mathbf{m}}$. Considerando os valores absolutos para $\xi_{\mathbf{m}}$ e $\lambda_{\mathbf{m}}$, podemos introduzir a integral de Melnikov na forma

$$
\int_{-\infty}^{+\infty} \cos \left(\left|\xi_{\mathbf{m}}\right| \psi_{1}^{s x}(\tau) \pm\left|\lambda_{\mathbf{m}}\right| \tau\right) d \tau=\frac{1}{\Omega_{G}} A_{2\left|\xi_{\mathbf{m}}\right|}\left(\mp\left|\lambda_{\mathbf{m}}\right|\right)
$$

onde $A_{2\left|\xi_{\mathbf{m}}\right|}$ é a integral de Melnikov com argumento $\pm\left|\lambda_{\mathbf{m}}\right|$. Assim, a integral em (6.35) é

$$
\int_{-\infty}^{+\infty} \sin \varphi_{\mathbf{m}}^{s x}(t) d t=\frac{1}{\Omega_{G}} \sin \varphi_{\mathbf{m}}^{0} A_{2\left|\xi_{\mathbf{m}}\right|}\left(\mp\left|\lambda_{\mathbf{m}}\right|\right)
$$

No outro ramo da separatriz, $\psi_{1}^{s x}$ possui sinal trocado, mas a simetria particular desta equação a torna invariante pela mudança do sinal de $\psi_{1}^{s x}$. Logo, obtemos o mesmo resultado (6.44). De fato, se $\psi_{1}^{s x}<0$, sendo o cosseno uma função par, a equação (6.43) pode ser escrita como

$$
\int_{-\infty}^{+\infty} \cos \left(\left|\xi_{\mathbf{m}}\right| \psi_{1}^{s x}(\tau) \mp\left|\lambda_{\mathbf{m}}\right| \tau\right) d \tau=\frac{1}{\Omega_{G}} A_{2\left|\xi_{\mathbf{m}}\right|}\left( \pm\left|\lambda_{\mathbf{m}}\right|\right),
$$

onde usamos $\psi_{1}^{s x}(\tau)=-\left|\psi_{1}^{s x}(\tau)\right|$. Portanto, as variações nas ações $p_{k}$ são obtidas introduzindo (6.44) em (6.35):

$$
\triangle p_{k} \approx \frac{\epsilon}{\Omega} \sum_{\mathbf{m} \neq \mathbf{m}_{\mathbf{G}}} \nu_{k}(\mathbf{m}) V_{\mathbf{m}}^{r} \sin \varphi_{\mathbf{m}}^{0}\left[A_{2\left|\xi_{\mathbf{m}}\right|}\left(\left|\lambda_{\mathbf{m}}\right|\right)+A_{2\left|\xi_{\mathbf{m}}\right|}\left(-\left|\lambda_{\mathbf{m}}\right|\right)\right]
$$


Chirikov fez uma estimativa da difusão usando a equação (6.46). Com o objetivo de simplificar a estimativa teórica da difusão, Chirikov considerou apenas perturbações ressonantes pares e desprezou a contribuição da perturbação com argumento negativo através da condição $\left|\lambda_{\mathbf{m}}\right| \gg 1$.

No caso do modelo de três corpos com movimentos médios ressonantes, as perturbações ressonantes não são pares, neste caso não é possível desprezar a contribuição da perturbação para a qual $\left|\lambda_{\mathbf{m}}\right|$ é pequeno. Portanto, cada perturbação contribui diferentemente quando o movimento está próximo a separatriz onde $\lambda_{\mathbf{m}}>0$ ou $\lambda_{\mathbf{m}}<0$. Se considerarmos perturbações ímpares tona-se necessário levarmos em conta que o tempo de permanência do movimento próximo à separatriz não são iguais. Isto é feito considerando que as soluções residem em apenas uma fração, do tempo total, próxima à separatriz com $\lambda_{\mathbf{m}}>0$. Para levarmos em conta essa assimetria introduzimos o fator $R_{T}$

$$
R_{T}=\frac{T_{\lambda}}{T}
$$

onde $T_{\lambda}$ é o tempo de permanência da solução próximo à separatriz com $\lambda_{\mathbf{m}}>0$ e $T$ é o tempo total. Então, podemos rescrever (6.46) como

$$
\triangle p_{k} \approx \frac{\epsilon}{\Omega} \sum_{\mathbf{m} \neq \mathbf{m}_{\mathbf{G}}} \nu_{k}(\mathbf{m}) Q_{\mathbf{m}} \sin \varphi_{\mathbf{m}}^{0}
$$

com

$$
Q_{\mathbf{m}}=V_{\mathbf{m}}^{r}\left[R_{T} A_{2\left|\xi_{\mathbf{m}}\right|}\left(\left|\lambda_{\mathbf{m}}\right|\right)+\left(1-R_{T}\right) A_{2\left|\xi_{\mathbf{m}}\right|}\left(-\left|\lambda_{\mathbf{m}}\right|\right)\right]
$$

A equação (6.48) é válida para perturbarções não-pares e $\lambda_{\mathbf{m}}$ pequeno. Com o objetivo de obtermos uma estimativa pra a equação (6.48) em termos de funções ordinárias devemos conhecer os valores de $\left|\lambda_{\mathbf{m}}\right|$ e $\mid \xi_{\mathbf{m}}$. Em geral, as relações para $A_{2\left|\xi_{\mathbf{m}}\right|}$ dependem do termo exponencial com argumento $\left|\lambda_{\mathbf{m}}\right|$.

\subsection{A taxa de difusão}

Na teoria de Chirikov, para difusão lenta, cada ressonância possui um papel na dinâmica do sistema. A ressonância principal, chamada de ressonância guia, define o domínio onde ocorre a difusão, i.é, ela cria a separatriz. A ressonância perturbadora mais forte é chamada 
"ressonância de camada. "Esta ressonância perturba a separatriz da ressonância guia e é responsável pela formação da camada estocástica e suas propriedades. Portanto, a ressonância de camada controla a dinâmica através da camada estocástica. As perturbações ressonantes mais fracas são ditas "ressonâncias condutoras". Estas perturbam a camada estocástica e controlam a dinâmica ao longo da camada estocástica. Logo, as ressonâncias condutoras são responsáveis pela deriva ao longo da camada estocástica, isto é, a difusão lenta.

\subsubsection{Tensor de Difusão}

Nosso objetivo é obtermos uma estimativa analítica para a difusão lenta. Para isto, vamos estimar a difusão nas ações cujas direções sejam dadas ao longo da camada estocástica. Introduzindo o tensor da difusão lenta

$$
D_{i j}=\frac{\overline{\triangle p_{i}(t) \triangle p_{j}(t)}}{T_{a}}, \quad i, j=3, \ldots, N
$$

onde

$$
T_{a}=\frac{\ln \left(32 e / \omega_{s}\right)}{\Omega_{G}}
$$

é o tempo característico do movimento, dentro da camada estocástica, da ressonância guia, igual a metade do período de libração ou igual a um período de circulação de $\psi_{1}$ próximo à separatriz, e a média no numerador é feita sob sucessivos valores de $\varphi_{\mathbf{m}}^{0}$, e $\omega_{s}$ é a largura da camada estocástica dada por

$$
\omega_{s}=-\frac{\omega^{r}}{\Omega_{G}^{2}} \omega_{\mathbf{m}_{\mathbf{L}}} \frac{\nu_{1}\left(\mathbf{m}_{L}\right) \nu_{2}\left(\mathbf{m}_{L}\right)}{\xi_{\mathbf{m}}} Q_{\mathbf{m}_{\mathbf{L}}}
$$

na última equação o subscrito $L$ indica a camada ressonante.

Da equação (6.48) segue que

$$
\overline{\triangle p_{i}(t) \triangle p_{j}(t)} \approx \frac{\epsilon^{2}}{\Omega^{2}} \sum_{\mathbf{m}_{\mathbf{D}}} \sum_{\mathbf{m}_{\mathbf{D}}^{\prime}} \nu_{i}\left(\mathbf{m}_{\mathbf{D}}\right) \nu_{j}\left(\mathbf{m}_{\mathbf{D}}^{\prime}\right) Q_{\mathbf{m}_{\mathbf{D}}} Q_{\mathbf{m}_{\mathbf{D}}^{\prime}} \overline{\sin \varphi_{\mathbf{m}_{\mathbf{D}}}^{0} \sin \varphi_{\mathbf{m}_{\mathbf{D}}^{\prime}}^{0}}
$$

$\mathrm{e}$

$$
2 \sin \varphi_{\mathbf{m}_{\mathbf{D}}}^{0} \sin \varphi_{\mathbf{m}_{\mathbf{D}}^{\prime}}^{0}=\cos \left(\varphi_{\mathbf{m}_{\mathbf{D}}}^{0}-\varphi_{\mathbf{m}_{\mathbf{D}}^{\prime}}^{0}\right)-\cos \left(\varphi_{\mathbf{m}_{\mathbf{D}}}^{0}+\varphi_{\mathbf{m}_{\mathbf{D}}^{\prime}}^{0}\right)
$$

É importante notar que as médias em (6.53) são feitas levando-se em conta: 
1. A correlação entre sucessivos valores de $\varphi_{\mathbf{m}}^{0}$ quando o sistema se aproxima das extremidades da câmada;

2. A possível interferência das ressonâncias condutoras.

Na construção do mapa, Chirikov expressa $\varphi_{\mathbf{m}}^{0}$ em termos da fase da camada ressonante $\varphi_{\mathbf{m}_{\mathbf{L}}}^{0}$, da seguinte forma

$$
\varphi_{\mathbf{m}_{D}}^{0}=r_{\mathbf{m}_{D}} \varphi_{\mathbf{m}_{L}}^{0}+b_{\mathbf{m}_{D}} t+d_{\mathbf{m}_{D}}
$$

aqui $t$ é medido em números de interações do mapeamento.

A interferência é importante se $r_{\mathbf{m}_{D}}=r_{\mathbf{m}^{\prime}{ }_{D}}$ e $b_{\mathbf{m}_{D}}=b_{\mathbf{m}^{\prime} D}$ para um par de ressonâncias condutoras. Neste caso, os termos que interferem devem ser somados de antemão e então tratados como um termo simples.

Com isso e usando (6.48) podemos escrever (6.53) como

$$
D_{i j}=\frac{\epsilon^{2}}{T_{a} \Omega_{G}^{2}} \sum_{\mathbf{m}_{D}} \nu_{i}\left(\mathbf{m}_{D}\right) \nu_{j}\left(\mathbf{m}_{D}\right) Q_{\mathbf{m}_{D}}^{2} \overline{\sin ^{2} \varphi_{\mathbf{m}_{D}}^{0}}
$$

\subsection{A aproximação de Estocasticidade Reduzida}

Agora queremos estimar $\overline{\sin ^{2} \varphi_{\mathbf{m}_{D}}^{0}}$. Para isto, necessitamos considerar que a estrutura da camada estocástica afeta o movimento do sistema. De fato, o estudo da teoria da difusão lenta tem mostrado que a camada estocástica é formada por duas regiões distintas. A primeira, mais central, próxima à separatriz não perturbada, é totalmente caótica. A segunda, mais externa, próxima à borda da camada estocástica, incluindo domínios de movimento regular formando ilhas de estabilidade. Quando a solução se aproxima da borda da camada estocástica, ela permanece na vizinhança destas ilhas de estabilidade por longo tempo. Este fenômeno é chamado de sticking, ele provoca uma redução na variação da difusão. Portanto, próximo a ilhas de estabilidade podem surgir correlações nas fases e nas variáveis que governam o movimento através e ao longo da camada estocástica. Neste caso, a evolução das fases $\varphi_{\mathbf{m}_{\mathbf{L}}^{0}}$ e $\varphi_{\mathbf{m}_{\mathbf{D}}^{0}}$ não podem ser simultaneamente aleatórias, e estas correlações diminuem a taxa da difusão. 
Para expressar a correlação entre $\overline{\sin ^{2} \varphi_{\mathbf{m}_{D}}^{0}}$ e $\overline{\sin ^{2} \varphi_{\mathbf{m}_{L}}^{0}}$, Chirikov usou a chamada aproximação da estocasticidade reduzida (ver Chirikov (1979)). Com isto a equação (6.56) tornase

$$
D_{i j}=\frac{\epsilon^{2}}{2 T_{a} \Omega_{G}^{2}} \sum_{\mathbf{m}_{D}} R_{\mathbf{m}_{D}} \nu_{i}\left(\mathbf{m}_{D}\right) \nu_{j}\left(\mathbf{m}_{D}\right) Q_{\mathbf{m}_{D}}^{2}, \quad i, j=3, \ldots, N .
$$

A equação (6.57) é uma estimativa teórica para a difusão dentro da camada estocástica. Os parâmetros $R_{T}$ e $R_{\mathbf{m}_{D}}$ correspondem a perturbações não-pares e a aproximação da estocasticidade reduzida, respectivamente. 
Capítulo

\section{Aplicação da Teoria de Chirikov e Modelos Numéricos}

Neste capítulo descrevemos os modelos numéricos usados na aplicação da teoria de Chirikov, vista no capitulo (6), ao Hamiltoniano do sistema exoplanetário GJ581. Além disso, mostramos os resultados para difusão em semieixo e excentricidade.

\subsection{Aplicação da Teoria de Chirikov ao Sistema GJ581}

Usamos as seguintes unidades para os vários parâmetros: as massas são dadas em massas da Terra, as frequências são dadas em radianos por ano e os períodos são dados em anos. Para aplicarmos a teoria descrita no capítulo (6) ao sistema GJ581, começamos adicionando ao Hamiltoniano (2.36), a parte secular $D_{1}, D_{2}$ e $D_{3}$ até segunda ordem em excentricidades, a fim de evitarmos a degenerescência. Assim, o desenvolvimento da formulação de Chirikov para o Hamiltoniano (2.35) será feita considerando a parte ressonante da função perturbadora e três termos da parte secular, dados por

$$
\begin{gathered}
D_{1}=1.64 \times 10^{-12}\left(1.2+0.8776 \frac{\left(2 L_{2}-P_{2}\right) P_{2}}{L_{2}{ }^{2}}+0.8776 \frac{\left(2 L_{3}-P_{3}\right) P_{3}}{L_{3}{ }^{2}}\right) L_{3}{ }^{-2} \\
D_{2}=2.811 \times 10^{-13}\left(1.05+0.12 \frac{\left(2 L_{1}-P_{1}\right) P_{1}}{L_{1}{ }^{2}}+0.12 \frac{\left(2 L_{2}-P_{2}\right) P_{2}}{L_{2}{ }^{2}}\right) L_{2}{ }^{-2}
\end{gathered}
$$

$\mathrm{e}$

$$
D_{3}=1.63 \times 10^{-12}\left(1.03+0.052 \frac{\left(2 L_{1}-P_{1}\right) P_{1}}{L_{1}{ }^{2}}+0.052 \frac{\left(2 L_{3}-P_{3}\right) P_{3}}{L_{3}{ }^{2}}\right) L_{3}{ }^{-2} .
$$

Aplicamos ao Hamiltoniano (2.35) a transformação canônica definida por 


$$
\begin{gathered}
\mathbf{I}^{\prime}=\left(L_{1}, L_{2}, L_{3}, P_{1}, P_{2}, P_{3}\right) \text { (ações) } \\
\boldsymbol{\theta}^{\prime}=\left(\tilde{\nu}_{1}, \tilde{\nu}_{2}, \tilde{\nu}_{3}, \varpi_{1}, \varpi_{2}, \varpi_{3}\right) \text { (ângulos) }
\end{gathered}
$$

As relações entre as novas e velhas variáveis são dadas por

$$
\begin{aligned}
& \tilde{\nu}_{1}=\lambda_{1} \\
& \tilde{\nu}_{2}=\lambda_{1}-2 \lambda_{2}-2 \lambda_{3} \\
& \tilde{\nu}_{3}=\lambda_{3}
\end{aligned}
$$

e

$$
\begin{aligned}
& L_{1}=\Lambda_{2}+\Lambda_{1} \\
& L_{2}=-2 \Lambda_{2} \\
& L_{3}=-2 \Lambda_{2}+\Lambda_{3} .
\end{aligned}
$$

Note que essa transformação nos permite reduzir o número de graus de liberdade, pois a perturbação ressonante não depende dos ângulos $\tilde{\nu}_{1}$ e $\tilde{\nu}_{3}$ separadamente, ou seja, são variáveis cíclicas. Assim, seus momentos conjugados, $L_{1}$ e $L_{3}$, podem ser tomados constantes.

Portanto, podemos escrever o Hamiltoniano (2.35) como

$$
H(\mathbf{I}, \boldsymbol{\theta})=H_{0}(\mathbf{I})+\tilde{V}(\mathbf{I}, \boldsymbol{\theta})
$$

onde

$$
\begin{gathered}
\mathbf{I}=\left(L_{2}, P_{1}, P_{2}, P_{3}\right) \\
\boldsymbol{\theta}=\left(\tilde{\nu}_{2}, \varpi_{1}, \varpi_{2}, \varpi_{3}\right) .
\end{gathered}
$$

Devemos observar que $H_{0}$ na equação (7.8) não é o mesmo que em (2.36). De fato, o $H_{0}$ da equação (7.8) é o Hamiltoniano (2.36) com a adição dos termos $D_{1}, D_{2}$ e $D_{3}$ dados por 
(7.1), (7.2) e (7.3), respectivamente. Além disso, é adicionado também o termo associado às frequências seculares $f_{1} P_{1}+f_{2} P_{2}+f_{3} P_{3}$, e sua expressão é dada por $(7.11)($ aqui para um valor de $\left.m_{2} \approx 2.8\right)$. O termo $\tilde{V}(\mathbf{I}, \boldsymbol{\theta})$ corresponde à perturbação.

$$
\begin{aligned}
& H_{0}=-0.01989694 L_{2}+0.003999900914-2.537041915 \times 10^{-13} \\
& \left(L_{2}+0.00002003274424\right)^{-2}-1.089599808 \times 10^{-14}{L_{2}}^{-2} \\
& -2.537041915 \times 10^{-13}\left(-2.0 L_{2}+0.00001286077102\right)^{-2} \\
& -0.00002602959572 P_{1}+0.0000434879322 P^{2}-0.0001905794971 P_{3} \\
& +1.636801236 \times 10^{-12}\left(1.2+0.2194000000 \frac{\left(-4.0 L_{2}-1.0 P_{2}\right) P_{2}}{L_{2}{ }^{2}}\right. \\
& \left.+0.8776 \frac{\left(-4.0 L_{2}+0.00002572154204-1.0 P_{3}\right) P_{3}}{\left(-2.0 L_{2}+0.00001286077102\right)^{2}}\right) \\
& \times\left(-2.0 L_{2}+0.00001286077102\right)^{-2} \\
& +7.029676175 \times 10^{-14}\left(1.05+0.12 \frac{\left(2.0 L_{2}+0.00004006548848-1.0 P 1\right) P_{1}}{\left(L_{2}+0.00002003274424\right)^{2}}\right. \\
& \left.+0.03000000000 \frac{\left(-4.0 L_{2}-1.0 \text { PQ }\right) P_{2}}{L_{2}^{2}}\right) L_{2}{ }^{-2} \\
& +1.636801236 \times 10^{-12}\left(1.03+0.052 \frac{\left(2.0 L_{2}+0.00004006548848-1.0 P_{1}\right) P_{1}}{\left(L_{2}+0.00002003274424\right)^{2}}\right. \\
& \left.+0.052 \frac{\left(-4.0 L_{2}+0.00002572154204-1.0 P_{3}\right) P_{3}}{\left(-2.0 L_{2}+0.00001286077102\right)^{2}}\right) \\
& \times\left(-2.0 L_{2}+0.00001286077102\right)^{-2}
\end{aligned}
$$

\subsection{Modelos Numéricos}

\subsubsection{O Mapa Simplético de Hadjidemetriou}

Sabemos que umas das principais propriedades dos sistemas Hamiltonianos é que sua função Hamiltoniana $H(\mathbf{p}, \mathbf{q})$ é uma integral primeira do sistema. Uma outra propriedade 
bastante importante é a que em tais sistemas o seu fluxo é simplético. Em particular, sabe-se que o fluxo no espaço de fase de dimensão $2 n$, de um ponto inicial $\left(\mathbf{p}_{0}, \mathbf{q}_{0}\right)$ em um tempo $t=t_{0}$ para um ponto final $\left(\mathbf{p}_{1}, \mathbf{q}_{1}\right)$ em um tempo $t=t_{1}$ é uma transformação simplética onde $n$ é o número de graus de liberdade dos sistema e $(\mathbf{p}, \mathbf{q}) \in \mathbb{R}^{2 n}$.

Definição 4. Um mapa $M: \mathbb{R}^{2 n} \rightarrow \mathbb{R}^{2 n}$ é dito simplético se

$$
D^{T} J D=J \quad \text { com } J=\left(\begin{array}{cc}
\mathbf{0} & \mathbf{I} \\
-\mathbf{I} & \mathbf{0}
\end{array}\right)
$$

onde $\mathbf{0}, \mathbf{I}$ e $\mathbf{D}$ são as matrizes zero e unidade $n \times n$ e a matriz Jacobiana de $M$, respectivamente.

Geometricamente, a simpleticidade do fluxo nos diz que ele preserva a área orientada no caso $n=1$. No caso geral $n>1$, a simpleticidade significa que a soma de áreas orientadas é a mesma após a transformação. Uma das principais ferramentas para o estudo de sistemas Hamiltonianos é o mapa de Poincaré ou superfície de seção. O fluxo no espaço de fase de dimensão $2 n$ é reduzido a um mapa no espaço de fase de dimensão $2 n-2$. Devemos notar que os pontos fixos do mapa de Poincaré coincidem com órbitas periódicas do sistema, consequentemente sua localização e estabilidade determinam a topologia do espaço de fase reduzido (ver, (Lichtenberg e Lieberman, 1992)). O mapa de Poincaré descreve completamente o sistema e é simplético. Porém, para encontrarmos o mapa de Poincaré devemos resolver equações diferenciais que, em geral, não podem ser resolvidas analiticamente, pois estão associadas a sistemas não integráveis. Assim, o mapa é obtido numericamente.

Como foi dito no capítulo (6), seção (6.4), ao invés de usarmos o mapa da separatriz usou-se o mapa simplético de Hadjidemetriou para se calcular a evolução das componentes do momento p. A construção deste mapa foi baseada no que foi feito por Hadjidemetriou (ver,Hadjidemetriou (1993),Ferraz-Mello (1996), Roig e Ferraz-Mello (1999)).

Consideremos um Hamiltoniano $H^{*}$ nas variáveis ação-ângulo ressonantes, com três graus de liberdade e dependente do tempo. Consideremos agora a variável tempo $t$ com uma nova variável e introduzamos seu momento conjugado $\varrho_{t}$. Com isso, obtemos um Hamiltoniano autônomo com quatro graus de liberdade $\bar{H}=H^{*}+\varrho_{t}$. Sejam $\boldsymbol{\theta}$ e $\mathbf{I}$ os quatro ângulos e as quatro ações, respectivamente. Então, o mapa simplético é definido 
pela transformação

$$
\left(\boldsymbol{\theta}^{n}, \mathbf{I}^{n}\right) \rightarrow\left(\boldsymbol{\theta}^{n+1}, \mathbf{I}^{n+1}\right)
$$

com a função geradora dada por

$$
S\left(\boldsymbol{\theta}^{n}, \mathbf{I}^{n}\right)=\sum_{i=1}^{4} \theta_{i}^{n} I_{i}^{n}+\tau \bar{H}\left(\boldsymbol{\theta}^{n}, \mathbf{I}^{n+1}\right)
$$

onde $\tau$ é o passo do mapa. O mapa de Hadjidemetriou é simplético por construção, e suas equações são dadas por

$$
\begin{aligned}
I_{i}^{n+1} & =I_{i}^{n}-\tau \frac{\partial H^{*}\left(\boldsymbol{\theta}^{n}, \mathbf{I}^{n+1}\right)}{\partial \theta_{i}^{n+1}} \\
\theta_{i}^{n+1} & =\theta_{i}^{n}+\tau \frac{\partial H^{*}\left(\boldsymbol{\theta}^{n}, \mathbf{I}^{n+1}\right)}{\partial I_{i}^{n+1}}, i=1,2,3
\end{aligned}
$$

e

$$
\varrho_{t}^{n+1}=\varrho_{t}^{n}-\tau \frac{\partial H^{*}\left(\boldsymbol{\theta}^{n}, \mathbf{I}^{n+1}\right)}{\partial t^{n}}, \quad t^{n+1}=t^{n}+\tau
$$

\subsubsection{Modelo Para Seis Ressonâncias}

Para o nosso modelo consideraram-se 6 ressonâncias: a ressonância guia(g), quatro ressonâncias condutoras(Cond) e a ressonância de camada(Cam). O mapa simplético de Hadjidemetriou (7.13) foi construído aplicando-se as equações (7.15) ao Hamiltoniano na variáveis de Chirikov. Para as seis ressonâncias acima o mapa nos dá as seguintes equações para as ações

$$
\begin{aligned}
& p_{1}^{n+1}=p_{1}^{n}+\tau\left(\zeta_{g} \sin \psi_{1}^{n}+\zeta_{\text {Cam }} \nu_{\text {Cam }} \cos \varphi_{\text {Cam }}^{n}+\zeta_{\text {Cond }} \nu_{\text {Cond }} \sin \varphi_{\text {Cond }}^{n}\right) \\
& p_{2}^{n+1}=p_{2}^{n}+\tau\left(\zeta_{\text {Cam }} \nu_{\text {Cam } 2} \cos \varphi_{\text {Cam }}^{n}+\zeta_{\text {Cond }} \nu_{\text {Cond }} \sin \varphi_{\text {Cond }}^{n}\right) \\
& p_{3}^{n+1}=p_{3}^{n}+\tau\left(\zeta_{\mathrm{Cam}} \nu_{\mathrm{Cam} 3} \cos \varphi_{\text {Cam }}^{n}+\zeta_{\mathrm{Cond}} \nu_{\mathrm{Cond}} \sin _{\mathrm{Cond}}^{n}\right) \\
& p_{4}^{n+1}=p_{4}^{n}+\tau\left(\zeta_{C a m} \nu_{C a m_{4}} \cos \varphi_{\text {Cam }}^{n}+\zeta_{\text {Cond }} \nu_{\text {Cond }} \sin \varphi_{\text {Cond }}^{n}\right)
\end{aligned}
$$

onde $\operatorname{Cond}=\sum_{j=1}^{4} \operatorname{Cond}_{j}$ e Cond $_{j}$ é cada uma das ressonâncias condutoras. Para os ângulos 


$$
\begin{aligned}
\psi_{1}^{n+1} & =\psi_{1}^{n}+\tau\left(\sum_{k=1}^{4} \frac{p_{k}^{n+1}}{M_{k 1}}\right) \\
\psi_{2}^{n+1} & =\psi_{2}^{n}+\tau\left(\sum_{k=1}^{4} \frac{p_{k}^{n+1}}{M_{k 2}}+\left\|\boldsymbol{\omega}^{r}\right\|\right) \\
\psi_{3}^{n+1} & =\psi_{3}^{n}+\tau\left(\sum_{k=1}^{4} \frac{p_{k}^{n+1}}{M_{k 3}}\right) \\
\psi_{4}^{n+1} & =\psi_{4}^{n}+\tau\left(\sum_{k=1}^{4} \frac{p_{k}^{n+1}}{M_{k 4}}\right)
\end{aligned}
$$

onde $\zeta_{m}$ são os coeficientes das respectivas ressonâncias, $\nu_{\mathbf{m}_{i}}$ são as componentes dos vetores $\boldsymbol{\nu}_{2}$ e $\boldsymbol{\nu}_{3}$ na nova base, $\varphi_{\mathbf{m}}=\boldsymbol{\nu}_{\mathbf{m}} \cdot \boldsymbol{\psi}^{n}$ e $\tau$ é o passo do mapa.

\subsection{Parâmetros Numéricos}

Como pode ser visto na seção (4.1.1) os coeficientes, dos multipletos da ressonância $(1,-2,-2)$, aparecem em segunda ordem nas massas do planeta, isto faz com que , dependendo do valor das massas alguns coeficientes decresçam, podendo até "desaparecer". Por outro lado, alguns outros coeficientes crescem de forma considerável. De fato, após vários experimentos, observou-se que para a existência de um planeta na Zona Habitável dois fatores são de suma importância:

- Cada ressonância do mutipleto produz uma superfície ressonante nos espaço das ações $\left(L_{1}, L_{2}, L_{3}, P_{1}, P_{2}, P_{3}\right)$ como mostra a figura (7.1). Fixou-se $L_{1}=L_{1}^{r}, L_{2}=L_{2}^{r}$, $L_{3}=L_{3}^{r}$, onde $L_{1}^{r}, L_{2}^{r}$ e $L_{3}^{r}$, são os valores ressonantes das ações $L_{1}, L_{2}$ e $L_{3}$, respectivamente. Com isso, as equações das superfícies ressonantes dependeriam apenas das ações $P_{1}, P_{2}$ e $P_{3}$. Assim, foi possível construir as superfícies ressonantes no espaço $\left(P_{1}, P_{2}, P_{3}\right)$. Além disso, devido à dependência de cada ação com a respectiva excentricidade, também tornou-se possível construir as superfícies ressonantes no espaço $\left(e_{1}, e_{2}, e_{3}\right)$ como podemos ver na figura (7.2).

Contudo, as massas dos planetas também participam com parâmetros desconhecidos nas relações das superfícies ressonantes. Foram feitos vários estudos para investigar o comportamento das superfícies ressonantes para determinado trio de massas $m_{1}, m_{2}$ 
e $m_{3}$. Neste estudo fixaram-se as massas $m_{1}$ e $m_{3}$ e variou-se a massa $m_{2}$. Observouse que para valores de $m_{2}$ acima de um determinado valor, não se obtêm valores reais para as excentricidades relativas a determinada superfície ressonante. De fato, das variáveis de (2.27), pode-se obter as excentricidades, em particular $e_{2}^{r}$, através da expressão

$$
e_{2}=\frac{\sqrt{2 L_{2}-P_{2}} \sqrt{P_{2}}}{L_{2}}
$$

A equação (7.19) mostra que existem condições para que dado $P_{2}^{r}$ forneça excentricidades reais quando se considera uma superfície ressonante qualquer. Se $P_{2}^{r}$ deixa de satisfazer estas condições a superfície ressonante deixa de existir no espaço das excentricidades. Este fato tornou possível "desligar" de forma natural algumas ressonâncias cujo os coeficientes eram muito altos. De fato, foi observado nos experimentos numéricos que essas ressonâncias faziam com que o planeta teste escapasse rapidamente da zona habitável. Portanto, optou-se por escolher uma massa $m_{2}$ para à qual essas ressonâncias mais fortes "naturalmente" fossem desligadas.

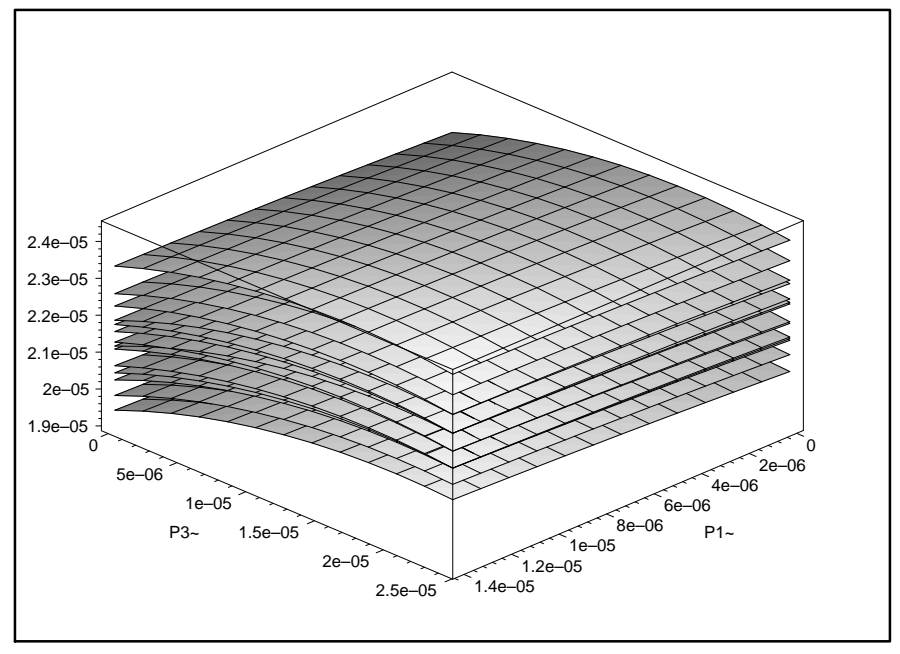

Figura 7.1: Superfícies ressonantes nos espaço das ações $\left(P_{1}, P_{2}, P_{3}\right)$.

- Um importante resultado obtido é que as excentricidades $e_{1}$ e $e_{3}$, dos planetas GJ581 c e d respectivamente, devem ser próximas a zero. Em outras palavras, suas órbitas devem ser quase circulares. Vale notar que existe uma grande discussão entre os observadores (ver Tadeu dos Santos et al. (2012)). Este resultado favoreceu os modelos correspondentes às soluções 3.1 e 3.2 de Tadeu dos Santos et al. (2012). 


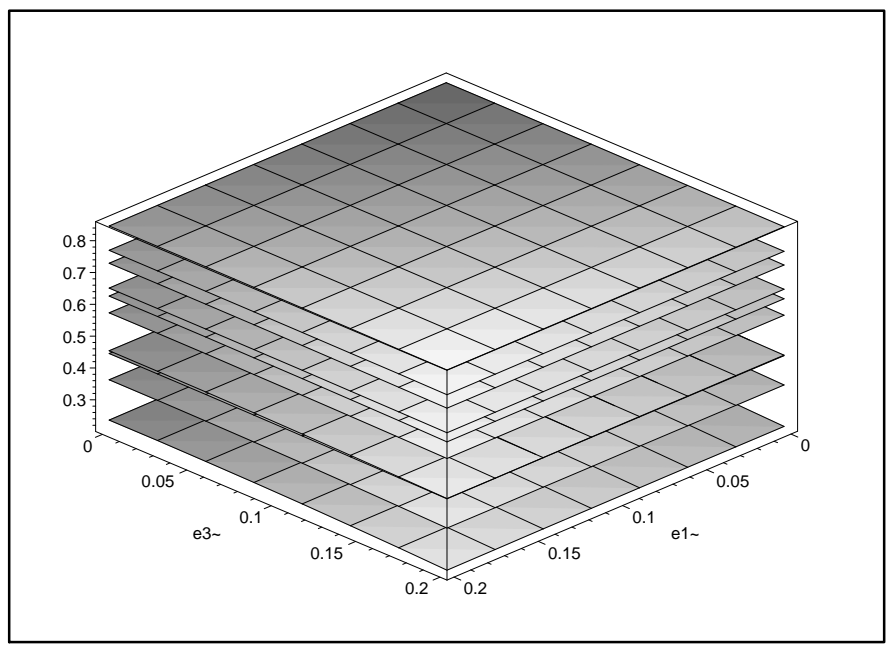

Figura 7.2: Superfícies ressonantes nos espaço das excentricidades $\left(e_{1}, e_{2}, e_{3}\right)$.

As massas escolhidas seguem os valores obtidos do artigo Tadeu dos Santos et al. (2012), com seus respectivos intervalos.

Como foi dito na seção (7.2.2), usamos o modelo com 6 ressonâncias, usando a nomenclatura de Chirikov, a ressonância guia $\mathbf{m}_{g}=(1,1,1)$, a ressonância de camada $\mathbf{m}_{\text {Cam }}=$ $(2,-1,2)$ e as ressonâncias condutoras $\mathbf{m}_{\text {Cond }_{1}}=(3,-1,1), \mathbf{m}_{\text {Cond }_{2}}=(3,0,0)$ e $\mathbf{m}_{\text {Cond }_{3}}=$ $(1,0,2)$ e $\mathbf{m}_{\text {Cond }_{4}}=(3,-2,2)$. Nestes tripletos os números se referem aos coeficientes da longitudes dos periélios, $\varpi_{1}, \varpi_{2}$ e $\varpi_{3}$.

A taxa de difusão foi calculada usando o procedimento de Chirikov, ou seja, foi usada a fórmula (5.53) de Chirikov (1979). A taxa de difusão é dada nas unidades $A U /$ tempo para semieixo e 1/tempo para excentricidades.

\subsection{Mapas de Contorno}

Nesta seção plotamos os mapas de contorno para o coeficiente de difusão em semieixo e excentricidade para alguns valores diferentes das massas $m_{1}$ e $m_{3}$.

\subsubsection{Massas $m_{1}=m_{3}=5$ Massas da Terra}

Para o semieixo, ou seja, difusão através do domínio estocástico observa-se que para o tempo de $10^{4}$ anos, ver figura (7.3), o movimento é bastante irregular; os valores do coeficientes de difusão mais significativos ocorrem dentro do domínio da ressonância guia. Fora deste domínio há um decrescimento do coeficiente de difusão e um aumento das regiões 
de regularidade. Para o tempo de $10^{5}$ anos o coeficiente de difusão cai significativamente ver figura (7.4), isto acontece porque as variações em semieixo alcançam seu valor máximo. Lembremos que os valores de semieixo são limitados pela largura da ressonância guia. De fato, a figura (7.4) mostra que o domínio estocástico é limitado e o valor do coeficiente de difusão cai em todo domínio considerado, porque o tempo total do experimento(que aparece na fórmula de Chirikov(5.5)) aumenta.

No caso da excentricidade, isto é difusão ao longo da camada estocástica, observa-se que para o tempo de integração de $10^{4}$ anos, a figura (7.5) concentra os maiores valores para o coeficiente de difusão, dentro do domínio da ressonância guia. Para o tempo de integração $10^{5}$ anos, a figura (7.6) mostra que o máximo coeficiente de difusão ocorre em uma região de baixa excentricidade dentro do domínio da ressonância guia, isto se deve à superposição das ressonâncias. Observa-se também um crescimento de regiões regulares.

A figura (7.7) mostra as separatrizes das ressonâncias guia $(G)$, camada(C) e condutoras. Note-se que as separatrizes das ressonâncias condutoras estão sobrepostas na linha vertical. Observa-se que o coeficiente de difusão é alto para baixas excentricidades, região onde há overlap das ressonâncias.

\subsubsection{Massas $m_{1}=5$ e $m_{3}=6$ Massas da Terra}

A figura (7.8) mostra que para um tempo de integração de $10^{3}$ anos, o coeficiente de difusão, para o semieixo, é máximo em quase toda região, exceto par pequenas "ilhas" onde o coeficiente de difusão é mínimo. Para um tempo de integração de $10^{4}$ anos, da figura (7.9) observa-se um considerável aumento da região de regularidade, e os valores máximos para o coeficiente de difusão fica restrito à região central dentro do domínio da ressonância guia, para baixas e altas excentricidades.

Em relação a excentricidade, a figura (7.10) mostra um ligeiro decrescimento da região de máximo coeficiente em contraste com o que acontece com o semieixo. Contudo, o coeficiente de difusão permanece alto dentro do domínio da ressonância guia para baixas e altas excentricidades, como mostra a figura (7.11)

A figura (7.12) segue o mesmo comportamento da figura (7.7). Contudo, observa-se um estreitamento do domínio estocástico. 


\subsubsection{Massa $m_{1}=5$ e $m_{3}=4$ Massas da Terra}

Para o semieixo, a figura (7.13) mostra que para um tempo de $10^{3}$ anos a região apresenta-se quase que totalmente caótica. Para um tempo de $10^{4}$ anos, da figura (7.14) nota-se que a região de máximo coeficiente de difusão tende a ficar restrita ao domínio estocástico.

No caso da excentricidade as figuras (7.15) e (7.16) mostram a região onde acontece o máximo coeficiente de difusão cai de forma considerável, com o aumento do tempo de integração. Pode-se ver também que a região de máximo coeficiente de difusão ocorre para valores de baixa excentricidade. Note-se que existe uma ilha de regularidade dentro do domínio estocástico para uma excentricidade próxima de 0.08, acima deste valor o coeficiente de difusão volta a crescer. A figura (7.17) mostra um a alargamento do domínio estocástico da ressonância guia em relação aos casos anteriores, figuras (7.7) e (7.17). 


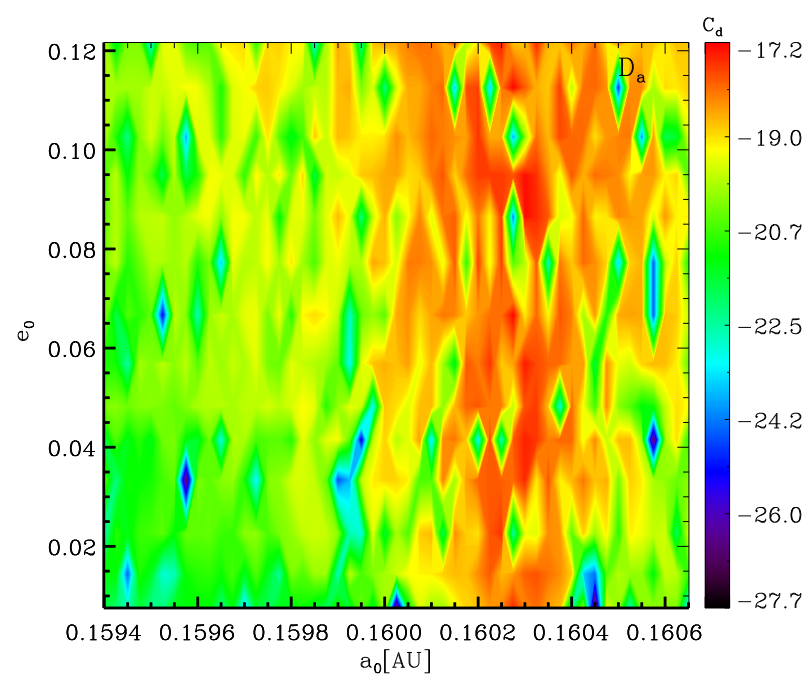

Figura 7.3: Difusão em semieixo para $t=10^{4}$ anos. $m_{1}=m_{3}=5 M_{\otimes}$.

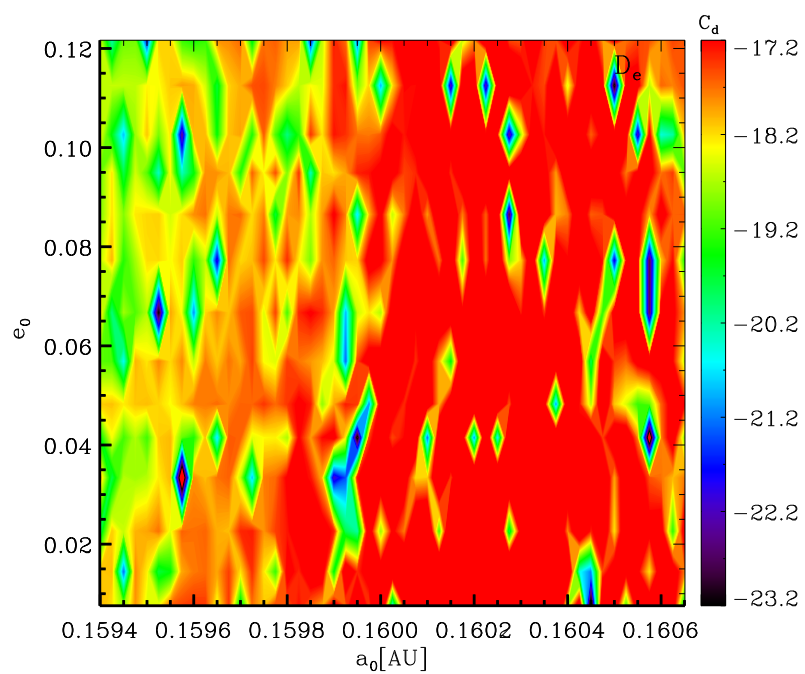

Figura 7.5: Difusão em excentricidade para $t=10^{4}$ anos. $m_{1}=m_{3}=5 M_{\otimes}$.

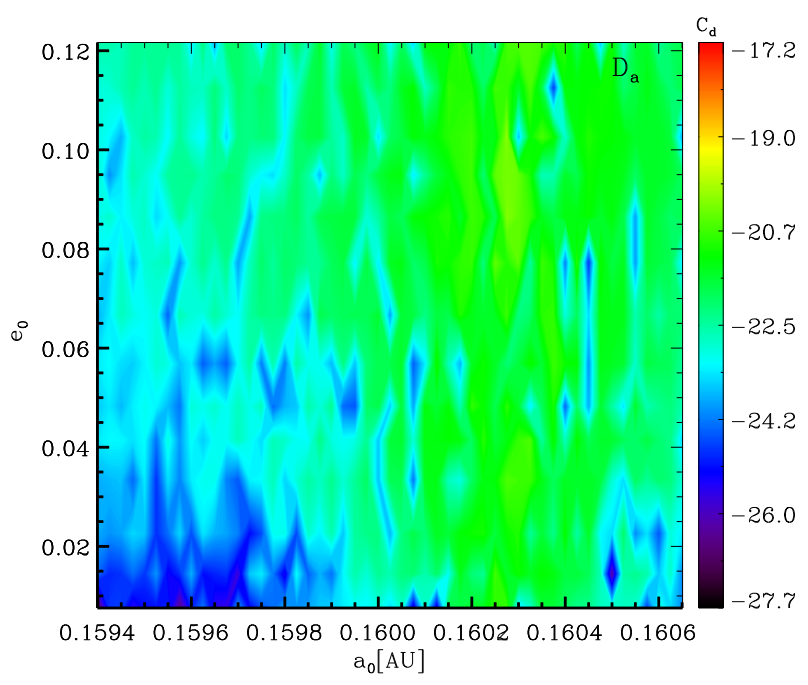

Figura 7.4: Difusão em semieixo para $t=10^{5}$ anos. $m_{1}=m_{3}=5 M_{\otimes}$.

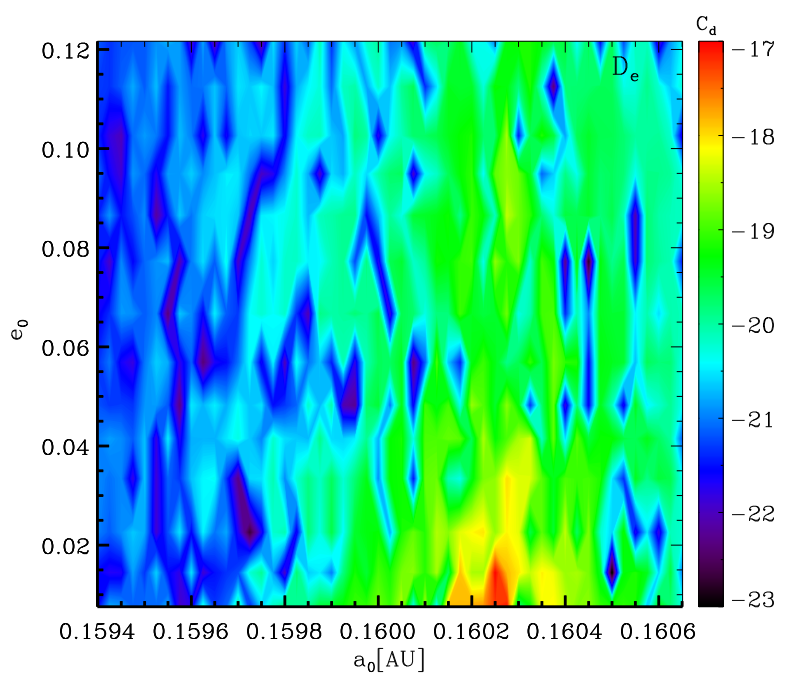

Figura 7.6: Difusão em excentricidade para $t=10^{5}$ anos. $m_{1}=m_{3}=5 M_{\otimes}$. 


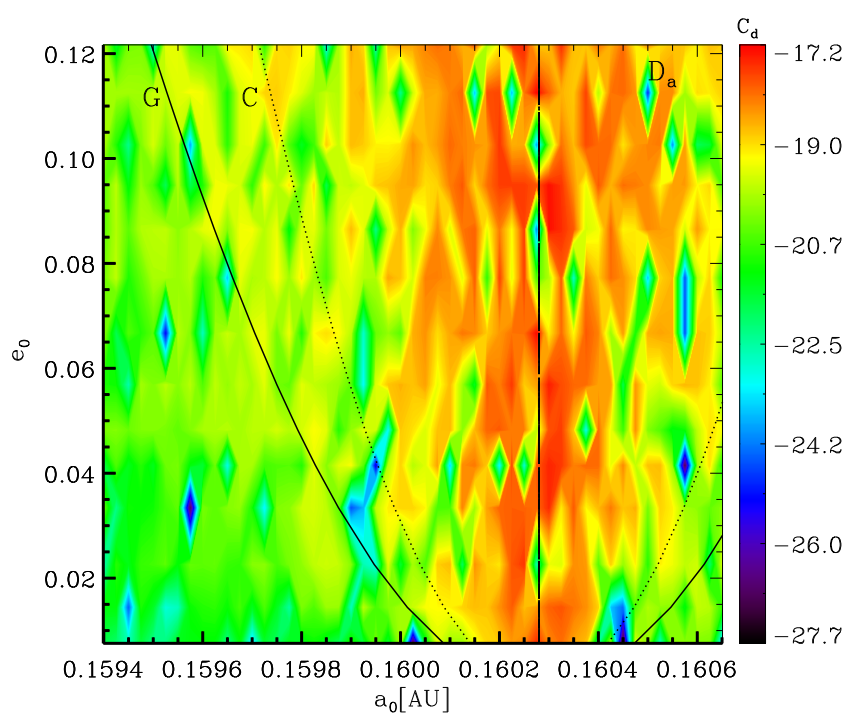

Figura 7.7: Separatrizes não perturbadas das ressonâncias guia $(\mathrm{G})$, camada $(\mathrm{C})$ para as massas $m_{1}=$ $m_{3}=5 M_{\otimes}$. As separatrizes das ressonâncias condutoras estão superpostas na linha vertical. Ao fundo tem-se o mapa de contorno para difusão em semieixo maior.
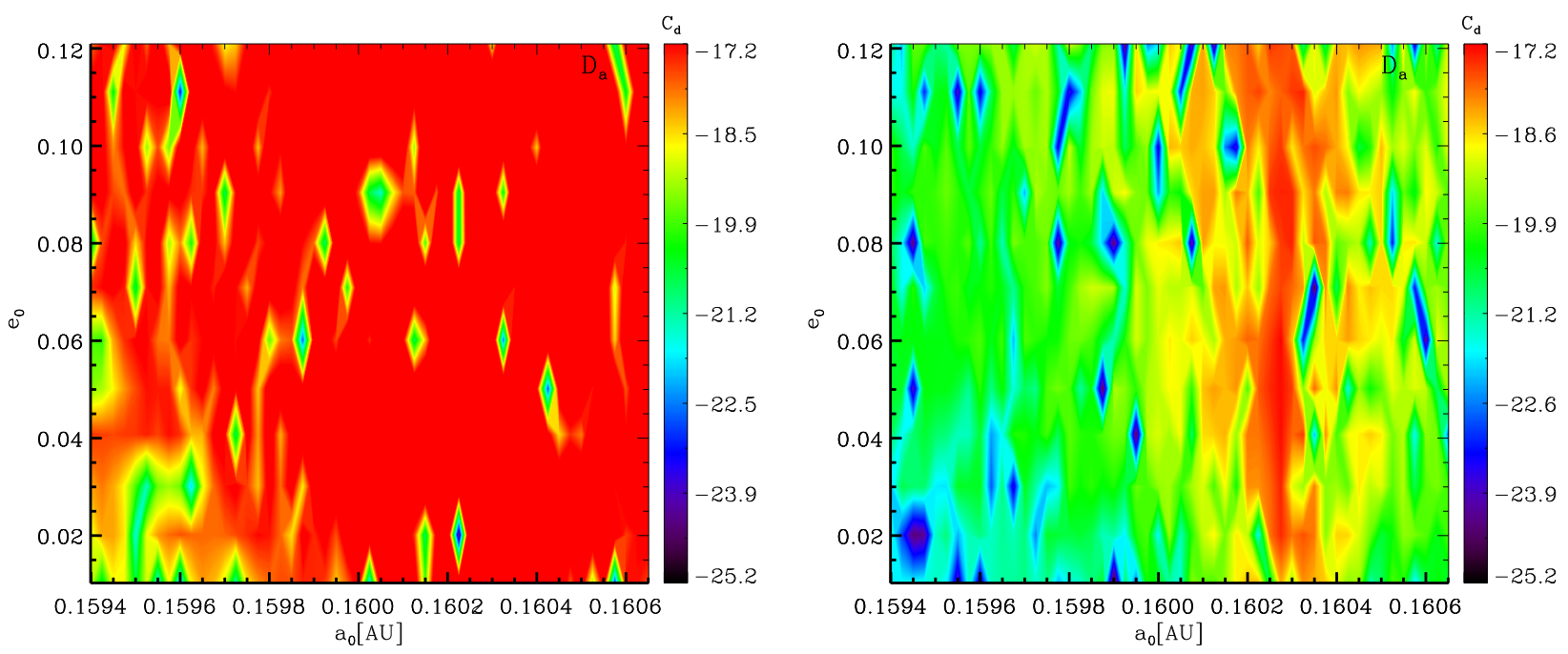

Figura 7.8: Difusão em semieixo para $t=10^{3} \cdot m_{1}=$ Figura 7.9: Difusão em semieixo para $t=10^{4} \cdot m_{1}=$ $5, m_{3}=6 M_{\otimes}$. $5, m_{3}=6 M_{\otimes}$. 

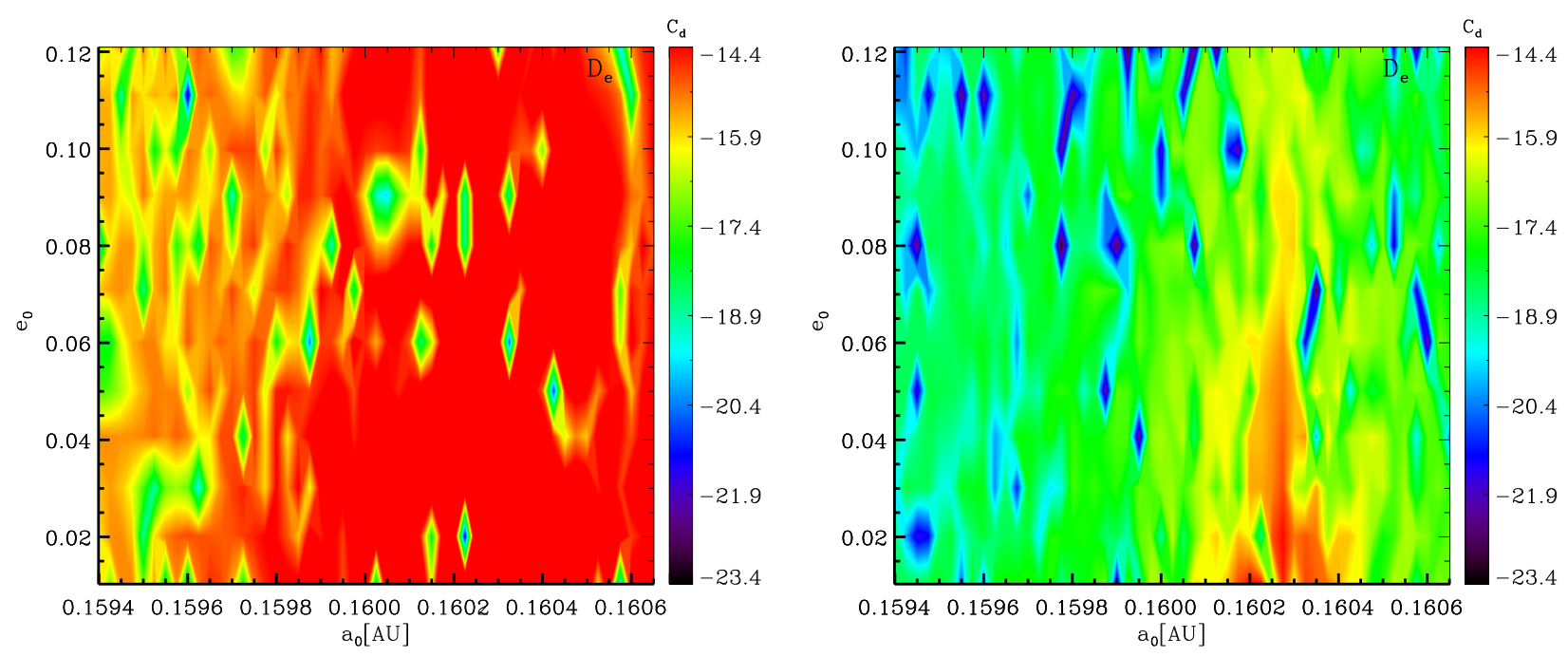

Figura 7.10: Difusão em excentricidade para $t=10^{3}$. $m_{1}=5 M_{\otimes}, m_{3}=6 M_{\otimes}$.

Figura 7.11: Difusão em excentricidade para $t=10^{4}$ anos. $m_{1}=5 M_{\otimes}, m_{3}=6 M_{\otimes}$.

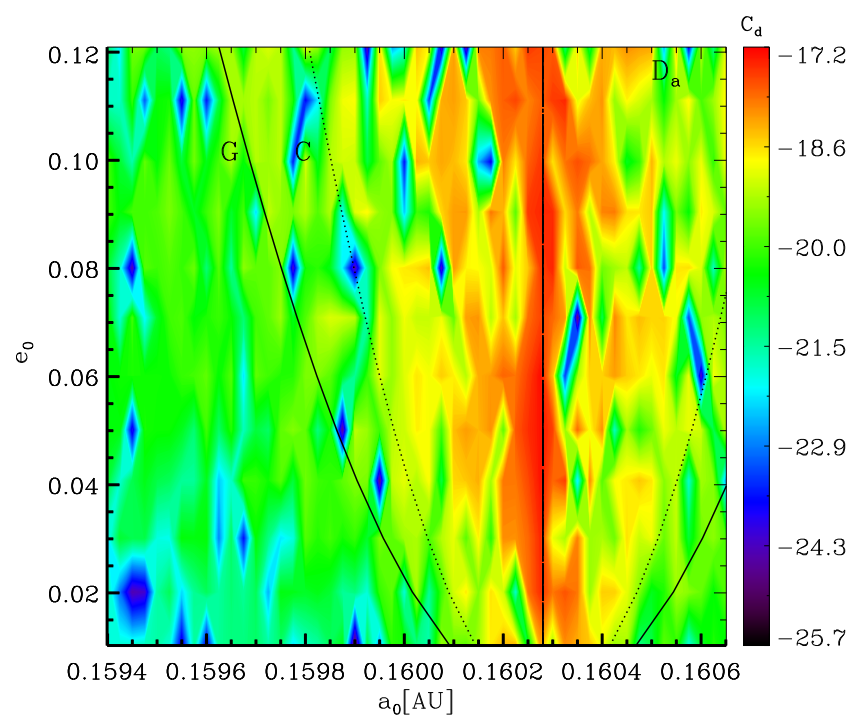

Figura 7.12: Separatrizes não perturbadas das ressonâncias guia $(\mathrm{G})$, camada $(\mathrm{C})$ para as massas $m_{1}=$ $5 M_{\otimes}, m_{3}=6 M_{\otimes}$. As separatrizes das ressonâncias condutoras estão superpostas na linha vertical. Ao fundo tem-se o mapa de contorno da difusão em semieixo maior. 


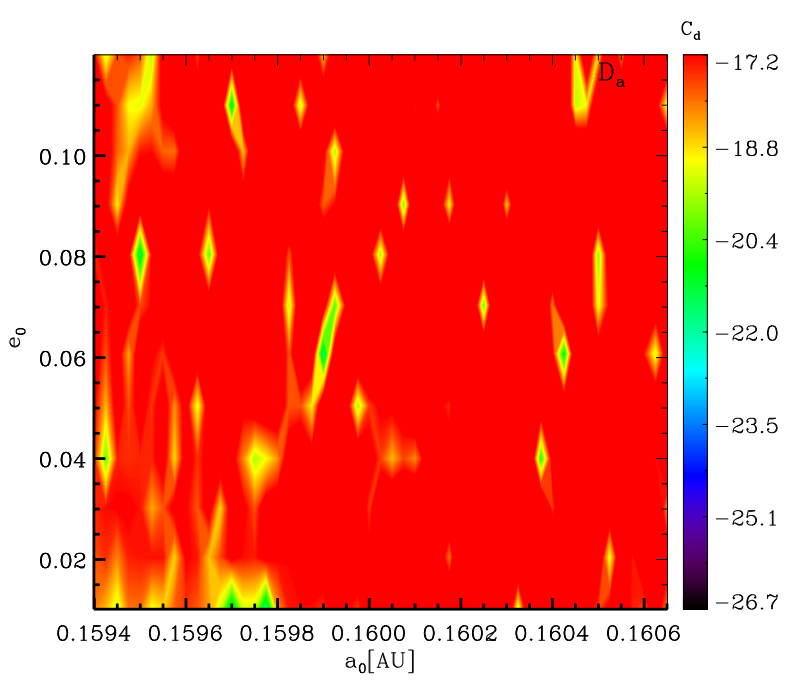

Figura 7.13: Difusão em semieixo para $t=10^{3}$ anos. $m_{1}=5 M_{\otimes}, m_{3}=4 M_{\otimes}$.

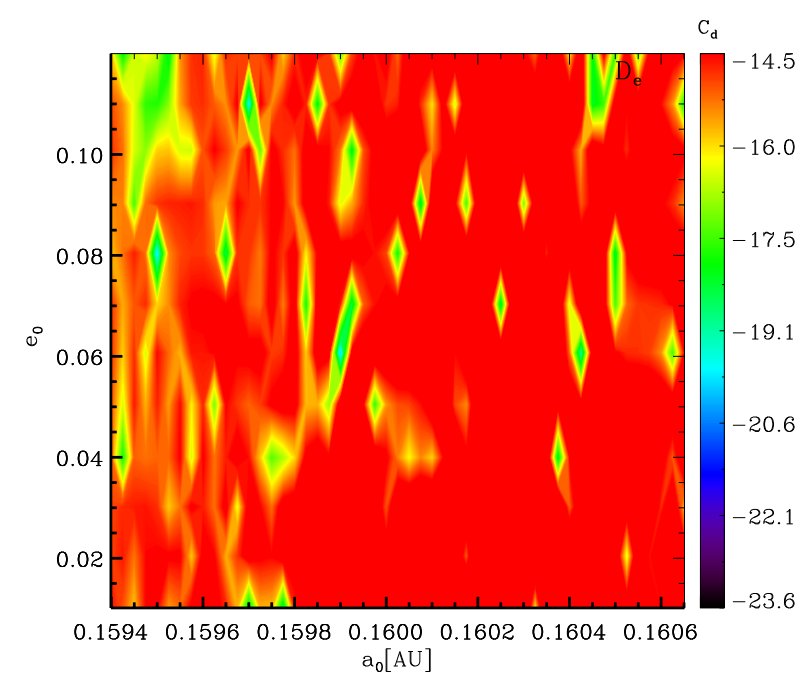

Figura 7.15: Difusão em excentricidade para $t=10^{3}$ anos. $m_{1}=5 M_{\otimes}, m_{3}=4 M_{\otimes}$.

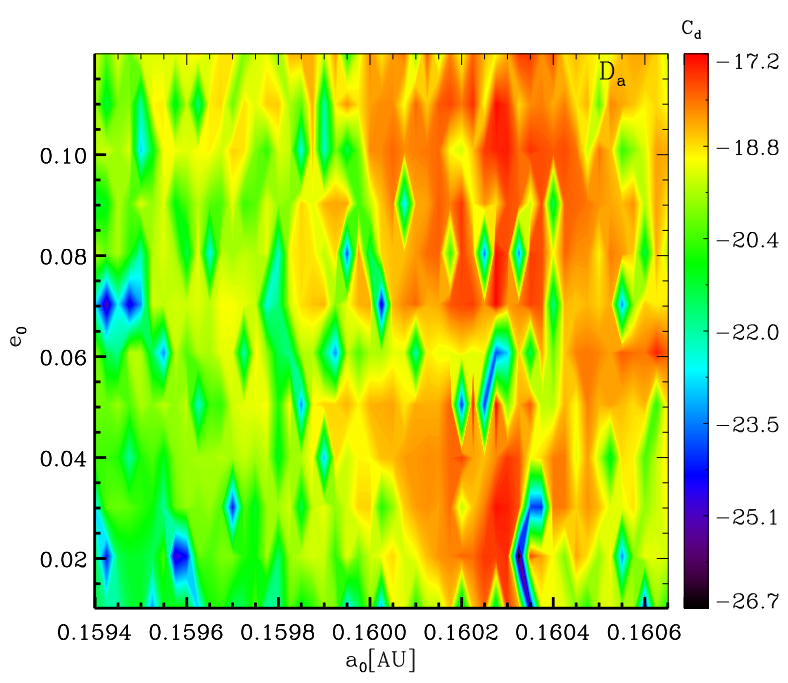

Figura 7.14: Difusão em semieixo para $t=10^{4}$ anos. $m_{1}=5 M_{\otimes}, m_{3}=4 M_{\otimes}$

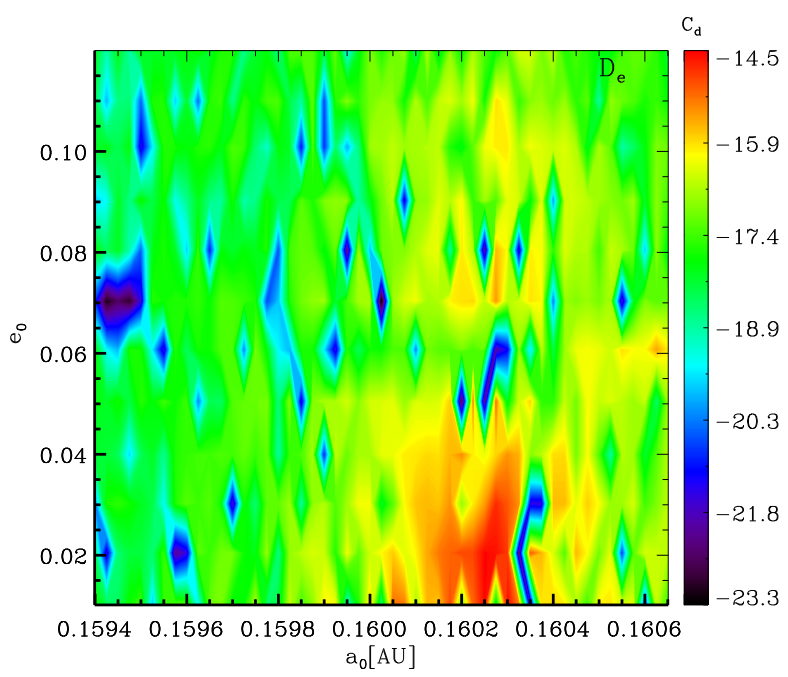

Figura 7.16: Difusão em excentricidade para $t=10^{4}$ anos. $m_{1}=5 M_{\otimes}, m_{3}=4 M_{\otimes}$. 


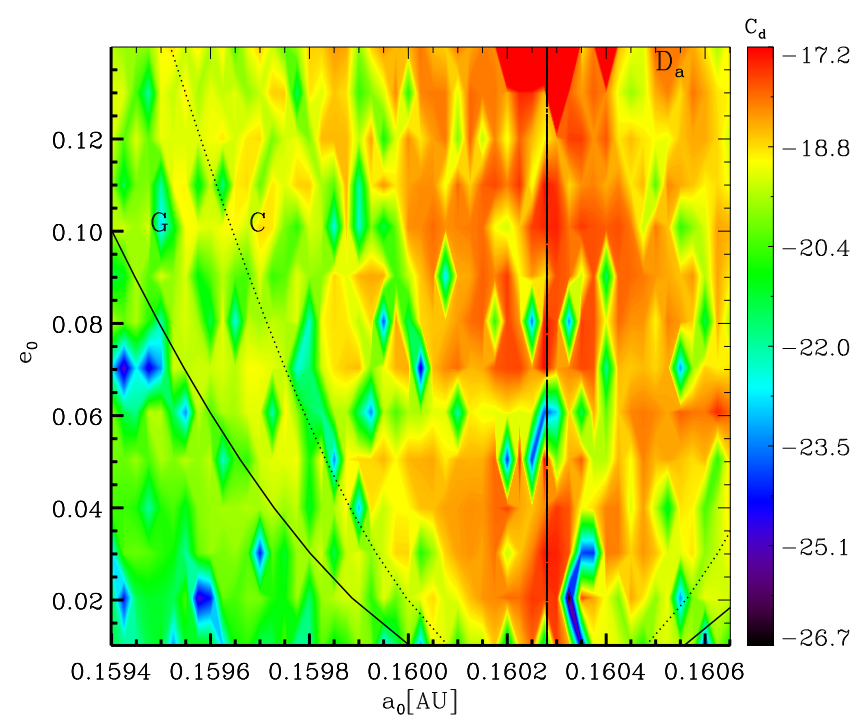

Figura 7.17: Separatrizes não perturbadas das ressonâncias guia $(\mathrm{G})$, camada $(\mathrm{C})$ paras as massas $m_{1}=$ $5 M_{\otimes}, m_{3}=4 M_{\otimes}$. As separatarizes das ressonâncias condutoras estão superpostas na linha vertical. Ao fundo mostra-se o mapa de contorno para difisão em semieixo maior. 


\section{Capítulo 8}

\section{Conclusões}

Uma análise geral deste trabalho permite concluir que as variáveis introduzidas por Chirikov, aplicadas ao Hamiltoniano para ressonâncias de três corpos do Sistema GJ581 obtido após a aplicação da teoria de Lie e com a utilização do mapa de Hadjidemetriou, são adequadas para o cálculo dos coeficientes de difusão em excentricidade e semieixo do planeta teste. A aplicação do modelo de Chirikov ao referido Hamiltoniano não foi de fácil implementação, devido ao grande número de parâmetros e ao comportamento dos coeficientes, dos multipletos da ressonância $(1,-2,-2)$, relacionados a estes parâmetros. Foram feito muitos experimentos até que se conseguisse obter resultados. Além disso, a transformação do Hamiltoniano para as variáveis ação-ângulo de Chirikov não é de fácil implementação, devido ao grande número de variáveis e parâmetros a serem estimados.

Observou-se que para a existência de um planeta na Zona Habitável devem-se ter valores convenientes para as massas dos planetas. Além disso, os planetas GJ581 c e d devem ter órbitas circulares(ou de muito baixa excentricidade).

Notou-se que a difusão em semieixo maior decresce com o aumento do tempo de mapeamento. Isto é característico da existência de barreiras confinando a difusão através da ressonância. No caso da excentricidade pode-se dizer que os valores altos da difusão nos tempos mais longos, mostram que a difusão ao longo da ressonância(ao contrário do que ocorre através da ressonância) não é confinada, e a difusão é alta mesmo para baixas excentricidades. Para o caso das massas $m_{1}=5$ e $m_{3}=4$ massas da Terra, observou-se que valor máximo para o coeficiente de difusão ocorre para baixas excentricidades $(e<0.04)$. Para valores de excentricidade maiores que 0.04 a região apresenta uma grande regularidade. As aplicações apresentadas mostram que o modelo é adequado para o estudo da difusão na ZH 
de GJ 581 e abre a possibilidade de uma extensa exploração da difusão nessa região. De fato, deve-se observar que temos uma grade relativamente "grossa" aproximadamente 1500 pontos, para os valores de semieixo e excentricidade iniciais, com o aumento do número de pontos na grade deve ser possível ver melhor a camada estocástica. Além disso, nessa exploração será importante usar outras ressonâncias como condutoras e de camada. Também será conveniente fazer-se mais experimentos para maiores tempos de mapeamento, fixandose também outros valores para as massas $m_{1}$ e $m_{3}$, visto que os valores destas massas afetam bastante os coeficientes das ressonâncias. 
Apêndice 

Apêndice A

\section{Resultados Básicos de Sistemas Dinâmicos}

\section{A.1 Sistemas Integráveis}

Definição 5. Um sistema com $N$ graus de liberdade é dito integrável, se existem $N$ integrais ou constantes do movimento.

Observação 1. Devemos notar que tais integrais não devem necessariamente ter uma forma analítica conhecida, elas devem existir.

Observação 2. Sabemos que para um sistema com $N$ graus de liberdade, integrável, o movimento no espaço de fase, de dimensão $2 N$, deve estar confinado ao subespaço do espaço de fase, de dimensão $2 N-N$. Em outras palavras, as $N$ constantes de movimento reduzem o número de graus de liberdade, a dimensão do sistema, por $N$.

Supondo que o movimento é periódico, e portanto ocupando um volume finito do espaço de fase, existe um importante teorema que nos afirma:

Teorema 4. O subespaço de $N$-dimensão, do espaço de fase de $2 N$-dimensão, não tem a topologia de uma $N$-esfera mas ao invés disso ele tem a topologia de um $N$-toro.

Topologicamente a $N$-esfera e o $N$-toro são diferentes, pois não podemos mapear o toro na superfície da esfera sem quebra-lo.

Observação 3. Curvas topologicamente equivalentes são quelas que podem ser mapeadas uma na outra ou reduzidas a um ponto.

No caso particular de $N=2$, se pensarmos no campo de vetores das trajetórias de fase tangentes como fios de cabelo, não há como pentearmos os cabelos na superfície de 
uma esfera sem termos uma singularidade, onde o campo de vetores não está definido. A direção do fio de cabelo que estar no polo não está definida. Isto é conhecido como Teorema "Hairy Ball", informalmente, e de maneira rigorosa como Teorema de Poincaré-Hopf.

Note que esta singularidade não aparece se as trajetórias movem-se na superfície de um toro

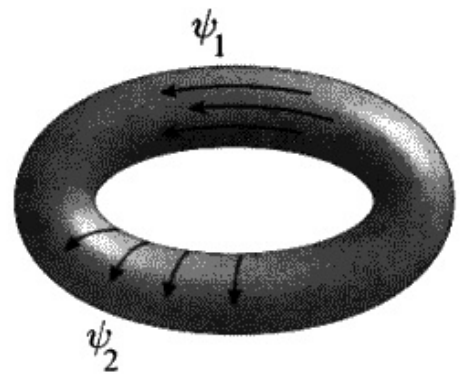

Observemos que no caso $N=2$, um possivel espaço de fase é dado pelas variáveis Ação-ângulos $I_{1}, I_{2}, \psi_{1}, \psi_{2}$, o movimento é duas vezes periódico, em $\psi_{1}$ e $\psi_{2}$. De fato, as variáveis angulares $\psi_{1}$ e $\psi_{2}$, são tais que

$$
\begin{aligned}
& \psi_{1}+2 \pi=\psi_{1} \\
& \psi_{2}+2 \pi=\psi_{2}
\end{aligned}
$$

Observação 4. Mesmo que o problema integrável possa ser reduzido a quadratura, em princípio. Todavia, não é suficiente que existam $N$ constantes de movimento para $N$ graus de liberdade, estas constantes devem está em involução duas à duas.

\section{A.2 Convexidade de $H_{0}$}

A estabilização não- linear, para uma perturbação ressonante depende da massa $M$ no modelo do pêndulo para ressonância. Ao contrário, num sistema com 1.5 graus de liberdade pode acontecer que para um oscilador multi-dimensional a massa $M=\infty$, ou a efetiva nãolinearidade $\frac{1}{M}=0$. Se isso acontecer, Já não existirá uma estabilização não-linear. Para entender este caso de outra maneira, note que a direção da fase de oscilação no $\omega$-espaço é ao longo do vetor $(\partial \omega / \partial I, \mathbf{m})$, enquanto que a normal para um plano ressonante é paralela ao vetor $\mathbf{m}$. Sob a condição $\frac{1}{M}=\mathbf{m},(\partial \omega / \partial I, \mathbf{m})=0$ uma trajetória anda ao longo do plano ressonante. Assim, o sistema não sairá da ressonância, então não haverá uma 
estabilização não-linear. A não linearidade não "funciona", o sistema não linear comportase como um isócrono. Chamaremos este caso especial de oscilações multi-dimensionais não-lineares quase-isócrono. Note que este último conceito refere-se ao Hamiltoniano $H_{0}$. De forma mais precisa, a condição $\frac{1}{M}=0$ é necessária mas não suficiente para garantir a quase-isocronicidade desde que a estabilização não-linear pode ocorrer devido a algum dos termos de maior ordem da expansão de $H_{0}$ em $p_{1}$. Um estudo geral desta estrutura foi feito por Nekhoroshev. Em particular, ele introduziu uma importante noção de hamiltonianos ingrimes. A definição exata desta noção é bastante complicada, mas essencialmente ela significa que a superfície de energia para um hamiltoniano ingrime é convexa em toda parte. Assim, o plano tangente a uma superfície de energia possui apenas um ponto de intersecção com esta, enquanto que para um Hamiltoniano não-ingrime o plano e a superfície podem se intercectarem sobre uma certa subsuperfície. Se a convexidade da superfície de energia já é garantida pelos termos quadráticos da expansão de $H_{0}$ em torno do ponto de tangência, o Hamiltoniano $H_{0}$ é chamado, por Nekhoroshev, quase-convexo. Esta última propriedade é mais forte que a condição $\frac{1}{M} \neq 0$ para o modelo do pêndulo ser aplicável.

A diferença é que para o modelo do pêndulo a convexidade pelos termos quadráticos ao longo do vetor ressonante $\mathbf{m}$, ou mais geralmente, ao longo de todos os vetores inteiros, é suficiente enquanto que a quase-convexidade significa a "plena " convexidade, i.é. ao longo de qualquer direção no plano tangente. Esta ligeira diferença é muito importante no caso das chamadas múltiplas ressonâncias.

Uma $k$ - múltipla ressonância aparece quando $k$ condições de ressonância do tipo

$$
\omega(\mathbf{I}) \cdot \mathbf{m}_{i}=0,
$$

$\operatorname{com} \mathbf{m}_{i}, \quad i=1, \ldots, k$ L.I. são satisfeitas simultaneamente, ou seja, para o mesmo vetor $\mathbf{I}^{r}$ ou $\omega^{r}$. Isto deve acontecer na interseção das $k$ superfícies ressonantes.

O Hamiltoniano pode ser reduzido de forma análoga ao caso de uma ressonância simples, isto é, $k=1$. Podemos até escrever exatamente o Hamiltoniano ressonante, que é sua expansão em $p_{k}$ :

$$
H_{r}(\mathbf{p}, \psi)=T \mathbf{p}+U(\mathbf{p}, \psi)
$$

onde

$$
T(\mathbf{p})=H_{0}\left(\mathbf{I}^{\mathbf{r}}+\mathbf{p}_{\mathbf{i}} \mu_{\mathbf{i j}}\right)-H_{0}\left(\mathbf{I}^{r}\right)+\Omega \mathbf{p}_{i} \nu_{i}
$$




$$
U=\epsilon \sum_{i=1}^{k} V_{m n} \cos \psi_{1} .
$$

Seja $\left(\mu_{i j}\right)$, a matriz da transformação, tal que todo $\mathbf{p}_{\mathbf{i}}$, para $i=1, \ldots, k$, descreva o movimento ao longo de um vetor particular $\mathbf{m}^{i}$, para a base ressonante, isto é, $\mu_{i j}=\mathbf{m}_{j}^{i}$. Assim, o Hamiltoniano depende de $k<N$ novas fases $\psi_{l}$, logo existirão apenas $N-k$ integrais ressonantes, ditos, $p_{l}=$ constantes, para $l=k+1, \ldots, N$, as quais podemos tomar todas iguais a zero. Assim, o Hamiltoniano

$$
H_{r}(\mathbf{p}, \psi)=T(\mathbf{p})+U(\mathbf{p}, \psi)
$$

descreve um sistema com $k$ graus de liberdade. Diferentemente do caso de uma ressonância simples o último sistema não é mais integrável, e pode exibir um movimento muito complicado, em geral estocástico.

Porém, e isto é de grande importância, o movimento considerado é sempre limitado para um Steep- Hamiltoniano, não perturbado, e uma perturbação suficientemente pequena. De fato, se as superfícies $H_{0}(\mathbf{I})=$ constante, são convexas, de modo a fazer a superfície $T(\mathbf{p})=$ constante, desde de que $\mathbf{I}(\mathbf{p})$ é linear como o termo $\Omega \mathbf{p}_{i} \nu_{i}$.Portanto, a única solução para $T(\mathbf{p})=0$ é a solução trivial, ou seja, $\mathbf{p}=\mathbf{0}$, o ponto de tangência. Assim, para $U \rightarrow 0$ a conservação da energia $H_{r}=$ constante, limita a oscilação em $\mathbf{p}$ e portanto também em $\mathbf{I}$. No caso oposto, para um Hamiltoniano quase-isócrono, a equação $T(\mathbf{p})=0$ determina um certo subespaço no espaço de movimento dos $p_{l}$, e nada impede de seguirmos uma trajetória, deste subespaço, indefinidamente longa. Se conservarmos, na expansão de $T(\mathbf{p})$ em (3.40), apenas os termos quadráticos como fizemos para uma ressonância simples, as oscilações de uma ressonância múltipla serão limitadas, se a energia cinética

$$
T(\mathbf{p})=\frac{p_{i} p_{j}}{M_{i j}}
$$

é de sinal definido.

Esta é exatamente a condição de quase-convexidade de Nekhoroshev, para garantir limitações para um movimento arbitrário próximo a uma ressonância múltipla para os termos quadráticos, apenas se for necessário. Contudo, para a energia cinética ser completamente de sinal definido, ou seja, se para todo $\mathbf{p}$ e não para apenas alguns deles, como sob a condição $M^{-1} \neq 0$, em uma ressonância simples. 


\section{A.3 Sistema Instável a Priori}

Sejam $(\mathbf{A}, \boldsymbol{\alpha}),(\beta, \phi)$ coordenadas que descrevem um sistema mecânico com l graus de liberdade. Suponhamos que:

$$
\begin{array}{r}
\mathbf{A} \in V \subset \mathbb{R}^{l-1} \\
\alpha \in \mathbb{T}^{l-1} \\
\beta \in \mathbb{R} \\
\phi \in \mathbb{T}^{1}
\end{array}
$$

onde $V$ é o fecho de algum aberto limitado e $\mathbb{T}^{s}$ é o toro de dimensão s. $\mathbb{T}^{s}$ pode ser pensado como $C_{1}^{s}=\left\{\right.$ produto de s círculos unitários no espaço complexo $C^{s}$ de dimensão $s$ \} através da identificação

$$
\psi=\left(\phi_{1}, \ldots, \phi_{s}\right) \in T^{s} \leftrightarrow \mathbf{Z}=\left(z_{1}, \ldots, z_{s}\right) \in C^{s}
$$

com

$$
z_{j} \equiv e^{i \phi_{j}}, \quad j=1, \ldots, s
$$

O sistema livre consiste de $l-1$ rotatores descritos pelo ângulo $\boldsymbol{\alpha}$ com momento conjugado A, e um Pêndulo descrito pelo ângulo $\phi$ e momento conjugado I.

A energia de oscilação do Pêndulo é dada por

$$
P_{0}(I, \mathbf{A}, \phi)=\frac{1}{2} \frac{\mathbf{I}^{2}}{\mathbf{J}_{0}(A)}+g(\mathbf{A}) \mathbf{J}_{0}(\mathbf{A})(\cos \phi-1)
$$

onde $J_{0}(\mathbf{A})$ é um conveniente momento de inércia e $2 \pi(\mathbf{A})^{-1}$ é período característico das pequenas oscilações, ou, $g(\mathbf{A})$ é o expoente de Lyapunov de um ponto fixo instável. O Hamiltoniano (A.2) é chamado Hamiltoniano padrão do pêndulo.

Observação: O movimento dos rotatores não será afetado pelas pequenas oscilações do pêndulo.

Um exemplo de um Hamiltoniano completo

$$
h_{0}=\frac{1}{2} \frac{\mathbf{A}^{2}}{R}+P_{0}(I, \mathbf{A}, \phi)
$$


onde $R$ é um outro momento de inércia.

De forma geral, consideremos consideremos um Hamiltoniano independente de $\boldsymbol{\alpha}$ como:

$$
H_{0}(I, \mathbf{A}, \phi, \mu)=h(\mathbf{A}, \mu)+P(I, \mathbf{A}, \phi, \mu)
$$

onde $P$ é um Hamiltoniano real analítico dependendo de um parâmetro $\mu$ e que descreve um pêndulo no seguinte sentido: Lembremos que no Retrato de fase de um pêndulo, as linhas de isoenergia, no espaço $(I, \phi)$, com $P=E$ são curvas contínuas fechadas com propriedades topológicas que podem mudar quando E varia. As linhas que separam as regiões cobertas por curvas do mesmo tipo(curvas que não contem pontos de equilíbrio e que podem ser deformadas uma na outra)são chamadas separatrizes. As separatrizes contém ao menos um ponto de equilíbrio e no máximo um número finito deles. Suponhamos que $P$ dependa explicitamente de $(\mu, \mathbf{A})$. Com isso, o retrato de fase também dependerá de $(\mu, \mathbf{A})$. Queremos que, para todos os valores de $\mathbf{A}$ de nosso interesse, o pêndulo P tenha um ponto de equilíbrio instável linear $\left(I_{\mu}(\mathbf{A}), \phi_{\mu}(\mathbf{A})\right)$, que é o único ponto da correspondente separatriz. Além disso, requeremos também que $I_{\mu}(\mathbf{A}), \phi_{\mu}(\mathbf{A})$, juntos com os expoentes de Lyapunov $g(\mathbf{A}, \mu)$, dependam analiticamente de $\mathbf{A} e \mu$.

De fato, o que objetivamos é que numa mudança completa de $\mathbf{A}$ seu ponto fixo instável, que selecionamos para nossa análise, dependa analiticamente de $\mathbf{A}$ e $\mu$ e não se misturem com outros pontos fixos, enquanto $\mathbf{A}$ e $\mu$ variam. Tal ponto é dito ponto de equilíbrio instável selecionado de $P$.

Com estas considerações, dizemos que (A.4) descreve uma à priori instável reunião de rotatores assistidos em suas rotações pelo pêndulo livre com selecionado ponto de equilíbrio. Sem perda de generalidade, suponhamos que o tal ponto de equilíbrio seja $(I, \phi)=(0,0)$ e a energia associada a ele seja $P=0$.

De fato, usando a transformação canônica gerada pela função

$$
S \equiv\left(\phi-\phi_{\mu}\left(\mathbf{A}^{\prime}\right)\right) I^{\prime}+I_{\mu} \sin \left(\phi-\phi_{\mu}\left(\mathbf{A}^{\prime}\right)\right)+\boldsymbol{\alpha} \cdot \mathbf{A}^{\prime}
$$

ou seja, 


$$
\begin{aligned}
I=\frac{\partial S}{\partial \phi} & =I^{\prime}+I_{\mu} \cos \left(\phi-\phi_{\mu}(\mathbf{A})\right) \\
\mathbf{A} & =\mathbf{A}^{\prime} \\
\phi^{\prime}=\frac{\partial S}{\partial I^{\prime}} & =\phi-\phi_{\mu}\left(\mathbf{A}^{\prime}\right) \\
\boldsymbol{\alpha}^{\prime}=\frac{\partial S}{\partial \mathbf{A}^{\prime}} & =\boldsymbol{\alpha}-\left[I^{\prime}+I_{\mu}\left(\mathbf{A}^{\prime}\right) \cos \left(\phi-\phi_{\mu}\left(\mathbf{A}^{\prime}\right)\right)\right] \frac{\partial \phi_{\mu}}{\partial \mathbf{A}^{\prime}}+\frac{\partial I_{\mu}}{\partial \mathbf{A}^{\prime}} \sin \left(\phi-\phi_{\mu}\left(\mathbf{A}^{\prime}\right)\right)
\end{aligned}
$$

Podemos gerar um novo Hamiltoniano do tipo (A.4), que tem a $(I, \phi)=(0,0)$ como ponto de equilíbrio instável selecionado.

Além disso, se $P(\mathbf{A}, \mu) \equiv P(0, \mathbf{A}, 0, \mu)$ podemos sempre definir $P$ como

$$
P-P(\mathbf{A}, \mu)
$$

consequentemente, alterar $h$. Portanto, a hipótese de que $P(0, \mathbf{A}, 0, \mu)=0$ não é uma restrição.

Motivados pela descrição acima, faremos as seguintes hipóteses:

1. O Hamiltoniano não perturbado $H_{0}$ tem a forma (A.4) e energia $P$, do pêndulo tem a origem como pronto de equilíbrio instável selecionado, onde $P$ assume o valor $0, \forall$ A e $\mu$ no domínio de definição de $H_{0}$; o expoente de Lyapunov associado, $g(\mathbf{A}, \mu)$ :

$$
\left.g^{2} \equiv\left[\left(\partial_{I \phi}^{2} P\right)^{2}-\partial_{I}^{2} P \cdot \partial_{\phi}^{2} P\right]\right|_{(I, \phi)=(0,0)}
$$

é limitado longe de zero quando $(\mathbf{A}, \mu)$ varia em seu domínio de definição.

2. As funções $h$ e $P$ são analíticas em seus domínios definição. Assim, elas são holomórficas em suas variáveis num domínio complexo $S_{\rho^{\prime}, \rho, \xi^{\prime}, \xi, \bar{\mu}}$

$$
\begin{array}{r}
S_{\rho^{\prime}, \rho, \xi^{\prime}, \xi, \bar{\mu}}=\left\{I, \mathbf{A}, \zeta, \mathbf{z}, \mu|||I| \leq \rho^{\prime}, \text { e } \exists \mathbf{a} \in V\right. \text { para o qual } \\
\left|A_{i}-a_{i}\right| \leq \rho e e^{-\xi^{\prime}}<|\zeta|<e^{-\xi}, \\
\left.e^{-\xi^{\prime}}<\left|z_{j}\right|<e^{-\xi},|\mu| \leq \bar{\mu},\right\}
\end{array}
$$

com $z_{j} \equiv e^{i \alpha_{j}}$ e $\zeta=e^{i \phi}$

3. Condições de não degenerescência válidas em $S_{\rho^{\prime}, \rho, \xi^{\prime}, \xi, \bar{\mu}}$ :

$$
\begin{array}{r}
\operatorname{det}\left(\partial_{\mathbf{A}}^{2} h\right) \neq 0 \\
\operatorname{det}\left(\left.\partial_{I, \phi}^{2} P\right|_{(I, \phi)=(0,0)}\right) \neq 0 \\
\partial_{\mathbf{A}} h \cdot\left(\partial_{\mathbf{A}}^{2} h\right)^{-1} \cdot \partial_{\mathbf{A}} h
\end{array}
$$


Definição 6. Um Hamiltoniano que satisfaz as três hipóteses acima é dito Hamiltoniano regular anisócrono livre à priori instável.

Obs. Tal Hamiltoniano é dito instável à priori, porque a hipótese da instabilidade é claramente construída na definição de sistemas livres.

Obs. Tais Hamiltonianos são muito comuns no estudo de ressonâncias de sistemas anisócronos.

Como exemplo, considere um sistema com l graus de liberdade, com Hamiltoniano livre $h$, da forma $h(\mathbf{A}, B)$, nas coordenadas ação-ângulos $\mathbf{A}, B, \boldsymbol{\alpha}, \lambda$ tal que a equação da ressonância seja simplesmente

$$
\partial_{B}(\mathbf{A}, B)=0
$$

Suponha que $B=B(\mathbf{A})$ seja a superfície ressonante. Então se, $\epsilon f(\mathbf{A}, \boldsymbol{\alpha}, B, \lambda)$ é uma perturbação, podemos encontrar coordenadas canônicas $\left(\mathbf{A}^{\prime}, \boldsymbol{\alpha}^{\prime} I, \phi\right)$, com as quais podemos descrever o movimento que acontece próximo a ressonância e o Hamiltoniano, nestas coordenadas, tem a forma (A.4), onde

$$
h(\mathbf{A}, \mu)=\left[h\left(\mathbf{A}^{\prime}, I\right)+\epsilon \bar{f}\left(\mathbf{A}^{\prime}, I\right)+\epsilon G_{p}\left(\mathbf{A}^{\prime}, I, \phi, \epsilon\right)+\mathbb{O}\left(\epsilon^{2}\right)\right]+\epsilon^{p} f_{p}\left(\mathbf{A}^{\prime}, I, \boldsymbol{\alpha}^{\prime}, \phi, \epsilon\right)
$$

onde $G_{p}=\tilde{f}\left(I, \mathbf{A}^{\prime}, \phi\right)+\mathbb{O}(\epsilon)$ é a média da função $f-\bar{f}$ sob o ângulo $\boldsymbol{\alpha}$ apenas e $\bar{f}$ é a média de $f$ sob os ângulos $\boldsymbol{\alpha}$ e $\lambda ; p$ é pode ser fixado arbitrariamente e $\epsilon$ é a "força"da perturbação. É importante notar que, para valores de p relativamente grandes é difícil encontrar funções $G_{p}, f_{p}$ e um sistema de coordenadas no qual (A.10) vale e a região do espaço de fase em torno da superfície ressonante, onde as novas coordenadas podem ser usadas para descrever o movimento, se torna menor.

Consideremos o Hamiltoniano $H$ como uma perturbação de um Hamiltoniano $H_{0}$, onde $H_{0}$ é como na definição (6):

$$
H=H_{0}(I, \mathbf{A}, \phi, \mu)+\mu f(I, \mathbf{A}, \phi, \boldsymbol{\alpha}, \mu)
$$

conde $f$ é holomórfica no domínio $S_{\rho^{\prime}, \rho, \xi^{\prime}, \xi, \bar{\mu}}$ definido em (A.8).

No que segue nos referiremos com frequência a expansão em séries de Fourier de $f$, dada por: 


$$
f(I, \mathbf{A}, \phi, \boldsymbol{\alpha}, \mu)=\sum_{\mathbf{v} \in \mathbb{Z}^{l-1}} f_{\mathbf{V}}(I, \mathbf{A}, \phi, \boldsymbol{\alpha}, \mu) e^{i \mathbf{V} \cdot \boldsymbol{\alpha}}
$$

Problema da Difusão. Dados $\mathbf{A}_{1}, \mathbf{A}_{2}$ com

$$
H_{0}\left(0, \mathbf{A}_{\mathbf{1}}, 0,0\right)=H_{0}\left(0, \mathbf{A}_{\mathbf{2}}, 0,0\right) .
$$

Podemos encontrar para todo $\mu$ suficientemente pequeno, mas diferente de zero, valores iniciais próximos à $\left(0, \mathbf{A}_{\mathbf{1}}, 0\right)$ nas variáveis $(I, \mathbf{A}, \phi)$ que, em um certo tempo $t(\mu)$, evolui para valores próximos à $\left(0, \mathbf{A}_{\mathbf{2}}, 0\right)$ ? Mais precisamente, podemos realizar um deslocamento de ordem $\mathbb{O}(1)$ nas variáveis $\mathbf{A}$ com uma perturbação de ordem $\mu$ tão pequena quanto se queira?

\section{A.4 Sistemas Livres: Caminho de Difusão}

Consideremos um nivel de energia fixo $E$ e $\mu=0$. Seja

$$
\begin{aligned}
\gamma:\left[s_{1}, s_{2}\right] & \longrightarrow \mathbf{A} \\
s & \longmapsto \mathbf{A}_{s}
\end{aligned}
$$

uma curva analítica por partes ligando $\mathbf{A}^{1}=\mathbf{A}_{s 1} \grave{a} \mathbf{A}^{2}=\mathbf{A}_{s 2}$, tal que, usando a notação em (A.4), podemos encontrar constantes $\tau$ e $t$ para os quais temos:

1. $H_{0}\left(0, \mathbf{A}_{s}, 0,0\right)=h\left(\mathbf{A}_{s}, 0\right) \equiv E, s \in\left[s_{1}, s_{2}\right]$

2. Se $\boldsymbol{\omega}_{s}=\partial_{\mathbf{A}} h\left(\mathbf{A}_{s}, 0\right)$ e se conjunto

$$
\Sigma(C)=\left\{s \in\left[s_{1}, s_{2}\right] \mid C(s)<C\right\}
$$

onde

$$
C(s)=\sup _{\mathbf{v} \neq \mathbf{0}, \mathbf{v} \in \mathbb{Z}^{l-1}}\|\mathbf{v}\|^{-\tau} \cdot\left\|\mathbf{v} \cdot \boldsymbol{\omega}_{s}\right\|^{-1}
$$

então existe uma curva $\gamma$, que depende de uma constante $\bar{K}>0$ tal que:

$$
\frac{1}{\left(s_{2}-s_{1}\right)} \cdot \boldsymbol{m e d}(\Sigma(C)) \geq 1-\bar{K}(D C)^{-1 / t}
$$

Se $D$ é o máximo de $\left\|\partial_{\mathbf{A}} h(\mathbf{A}, 0)\right\|$ na vizinhança da curva $\gamma$.

Definição 7. Se $\gamma$ é uma curva que tem as propriedades 1 e 2 acima, ela é chamada de um caminho de difusão. 


\section{A.5 Difusão de Arnold}

Para um sistema com várias ressonância Para sistemas com três graus de liberdade, camadas ressonantes próximas a separatriz não são isoladas, uma da outra, pelas superfícies KAM. Para dois graus de liberdade, a superfície de KAM, de duas dimensões, divide à superfície de energia tridimensional, no espaço de fase, num conjunto de regiões separadas, limitadas pelas superfícies KAM, muitas delas como linhas isolando regiões de um plano, como na figura (A.1) para maiores detalhes ver Lichtenberg e Lieberman (1992).

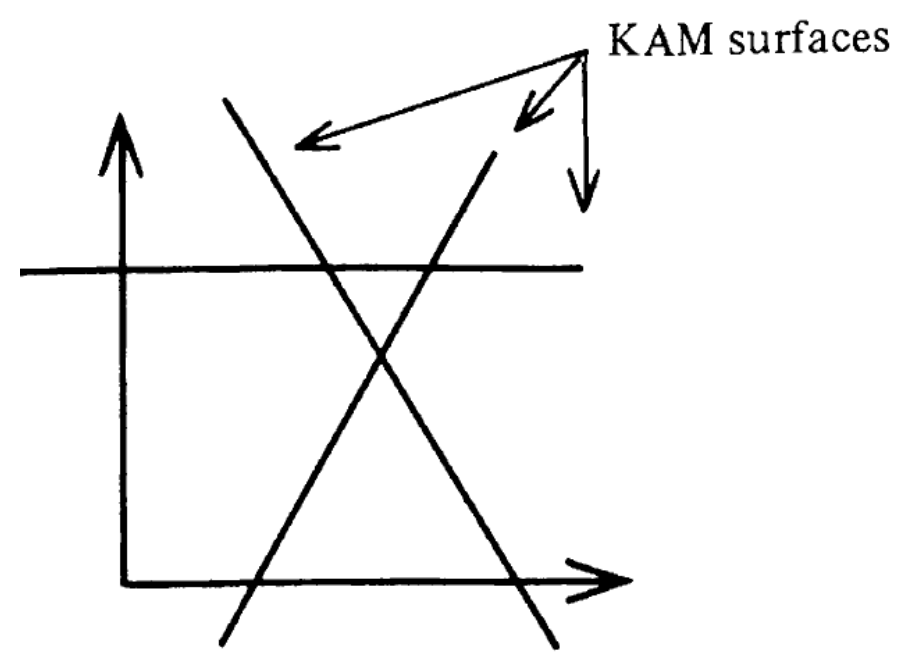

Figura A.1: Superfície de energia, representada por um plano, separada por retas em regiões isoladas, pelas superfícies KAM.

Para três graus de liberdade as superfícies KAM tridimensionais, não dividem a superfície de energia, agora de dimensão 5, em um conjunto de regiões separadas, analogamente como linhas não separam, um volume tridimensional em regiões distintas como na figura (A.2) Assim, de uma forma geral para $N>2$ graus de liberdade, as variedades $K A M$ de dimensão $N$ não divide a variedade de energia de dimensão $(2 N-1)$ em regiões distintas. Portanto, no caso genérico, todas as camadas estocásticas da variedade de energia no espaço de fase, estão conectadas numa complexa teia como na figura (A.3), chamada Teia de Arnold. Esta teia preenche todo espaço de fase intersectando-se ou situando-se perto de todos os pontos. Para condições iniciais dentro da teia, todo movimento estocástico seguinte, obrigatoriamente, intersectará todas as regiões finitas da superfície de energia no espaço de fase, mesmo no limite quando $\epsilon \rightarrow 0$. Este movimento é a Difusão de Arnold.

Do ponto de vista da pratica, duas questões relativas a difusão de Arnold são importantes: 


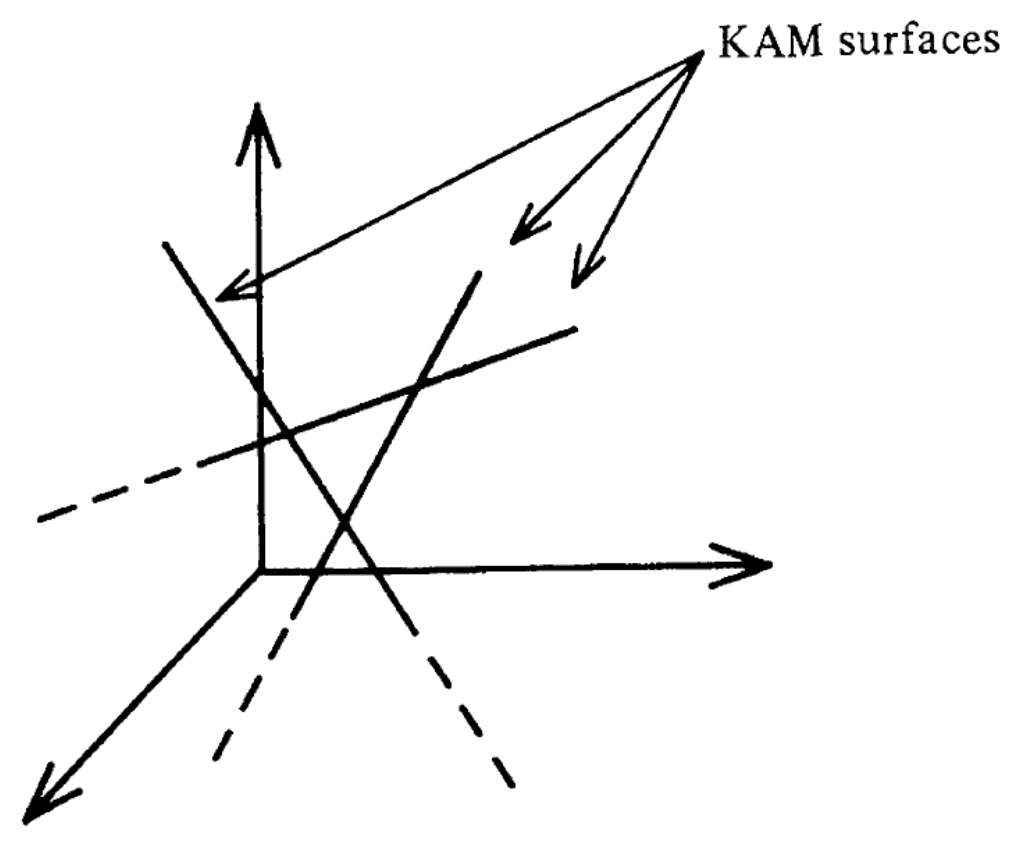

Figura A.2: Superfície de energia, representada pelo espaço, separadas por retas em regiões não isoladas, pelas superfícies KAM.

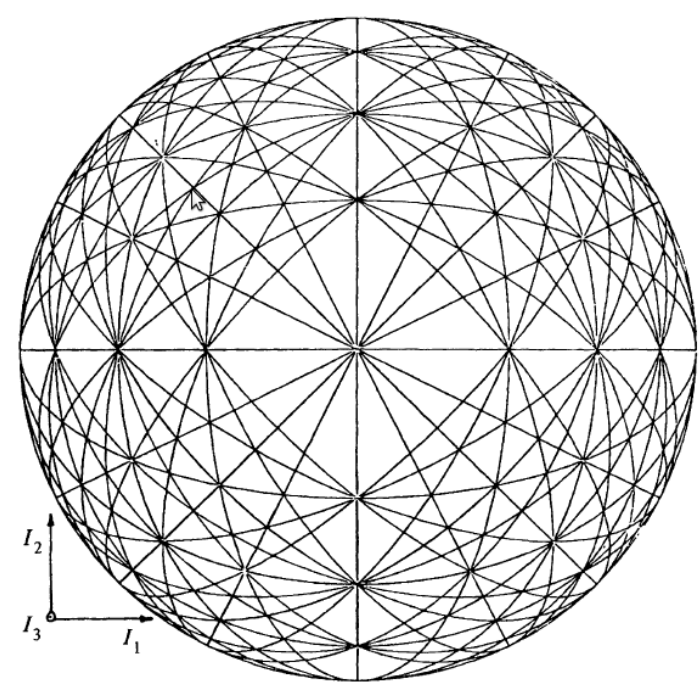

Figura A.3: Teia de Arnold para um Hamiltoniano de uma partícula livre.

1. Qual é a medida relativa das trajetórias estocásticas na região do espaço de fase de interesse?

2. Para uma dada condição inicial, quão rápido será difuso o sistema, ao longo dos fios finos da teia de Arnold? 


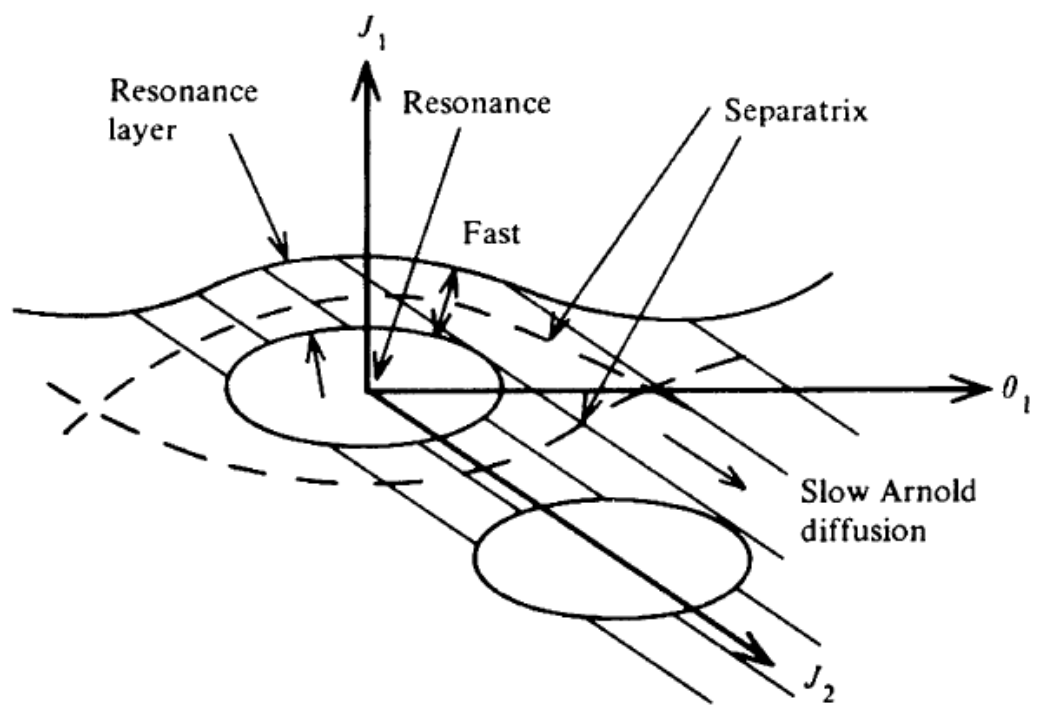

Figura A.4: Difusão ao longo e através da camada ressonante

\section{A.6 Sistemas Quase Integráveis e Estabilidade}

Consideremos um sistema Hamiltoniano com l graus de liberdade cuja função Hamiltoniana é

$$
H_{\epsilon}(\mathbf{I}, \phi)=h(\mathbf{I})+\epsilon f(\mathbf{I}, \phi)
$$

onde $(\mathbf{I}, \phi)=\left(I_{1}, \ldots, I_{l}, \phi_{1}, \ldots, \phi_{l}\right)$ pertencem ao espaço $V_{R} \times \mathbf{T}^{l}$ Dados $\nu_{1}, \ldots, \nu_{r} \in \mathbb{Z}^{l}$ L.I, seja $M=M\left(\nu_{1}, \ldots, \nu_{r}\right)$ o plano em $\mathbb{Z}^{l}$ gerado pelos vetores $\nu_{1}, \ldots, \nu_{r}$. Uma superfície ressonante, de ordem $r$, com o plano $M$ é a superfície

$$
\Sigma_{M}=\left\{\mathbf{I} \mid \mathbf{I} \in V_{R} ; \omega(\mathbf{I}) \cdot \nu_{j}=0, j=1, \ldots, r\right\}
$$

Sejam $r>0, N=\epsilon^{-r}$, com $\left\|\nu_{j}\right\| \leq N$, para $j=1, \ldots, r$. Neste caso dizemos que $M$ admite uma $N$-base.

Dada uma sequência $0<\lambda_{0}<\cdots \lambda_{l}$ e um plano $M \subset \mathbb{Z}^{l}$ admitindo uma $N$ - base, podemos definir a "região ressonante ou bloco ressonante" $B_{M}$ sendo uma vizinhança de $\Sigma_{M}$ consistindo de todos I's tais que, para ao menos uma $N$-base $\left\{\nu_{1}, \ldots, \nu_{r}\right\}$ de $M$, tem-se

$$
\left\|\omega(\mathbf{I}) \cdot \nu_{j}\right\|<\lambda_{r}, \quad j=1, \ldots, r
$$


e também, para $r<l$

$$
\|\omega(\mathbf{I}) \cdot \nu\| \geq \lambda_{r+1} \quad \forall \nu \text { nao pertencente a } M,\|\nu\| \leq N
$$

De maneira formal, denotando

$$
d_{M, N}(\mathbf{I})=\inf _{N-\text { Bases }} \max _{1 \leq j \leq r}\|\omega(\mathbf{I}) \cdot \nu\|
$$

tem-se

$$
B_{M}=\mathbf{I} \mid \mathbf{I} \in V_{R} ;\|\omega(\mathbf{I}) \cdot \nu\|>\lambda_{r+1}, \forall \nu \in M^{c},\|\nu\| \leq N ; d_{M, N}(\mathbf{I})<\lambda_{r}
$$

Note que, se $\lambda_{r+1}>2 \lambda_{r}$ o conjunto $U_{M}$ definido por

$$
U_{M}=\left\{\mathbf{I} \mid \mathbf{I} \in V_{R+1 / 2 \rho} ;\|\omega(\mathbf{I}) \cdot \nu\|>2 \lambda, \forall \nu \in M^{c},\|\nu\| \leq N\right\}
$$

contém $B_{M}$.

Definição 8. Uma função h é dita quase-convexa num conjunto $U$, se para qualquer $\mathbf{I} \in U$ $\operatorname{com} \omega(\mathbf{I}) \cdot \mathbf{v}=0$ e $h^{\prime \prime}(\mathbf{I}) \mathbf{v} \cdot \mathbf{v}=0$, então $\mathbf{v}=\mathbf{0}$

Proposição 5. Suponha que h, no Hamiltoniano (A.15), seja quase-convexa. Então:

1. Para qualquer $(\mathbf{I}(\mathbf{t}), \phi(t))$, com $\mathbf{I}(0) \in B_{M}$, tem-se

$$
\begin{array}{r}
(t) \in U_{M} \\
\|\mathbf{A}(t)-\mathbf{A}(0)\| \leq k \epsilon^{1 / 6} \\
\operatorname{para}|t|<T \equiv 1 / E\left(\frac{\epsilon}{\epsilon_{0}}\right)^{-3 / 4} e^{(1 / \epsilon)^{b} \xi(1 / 8)}
\end{array}
$$

2. Para cada plano $M$ existe um sistema de coordenadas canônicas $\left(\mathbf{I}^{\prime}, \phi^{\prime}\right)$ tal que $(\mathbf{I}, \phi)=\Psi_{M, \epsilon}\left(\mathbf{I}^{\prime}, \phi^{\prime}\right)$, $\Psi$ real analítica, tal que o Hamitoniano , nas novas coordenadas tem a seguinte forma

$$
H_{\epsilon}^{\prime}\left(\mathbf{I}^{\prime}, \phi^{\prime}\right)=h\left(\mathbf{I}^{\prime}\right)+\epsilon G\left(\mathbf{I}^{\prime}, \phi^{\prime}, \epsilon\right)+e^{-(1 / \epsilon)^{b} \xi(1 / 8)} f_{\infty}\left(\mathbf{I}^{\prime}, \phi^{\prime}, \epsilon\right)
$$

com

$$
G\left(\mathbf{I}^{\prime}, \phi^{\prime}, \epsilon\right)=\sum_{\nu \in M} G_{\nu}\left(\mathbf{I}^{\prime}, \epsilon\right) e^{i \nu \cdot \phi^{\prime}}
$$


onde

$$
G_{\nu}\left(\mathbf{I}^{\prime}, 0\right)=f_{\nu}\left(\mathbf{I}^{\prime}\right) \equiv \frac{1}{(2 \pi)^{l}} \int e^{-i \nu \cdot \phi} f\left(\mathbf{I}^{\prime}, \phi\right) d \phi
$$

denota as componentes de Fourier de $f$

Note que

$$
\dot{\mathbf{I}}^{\prime}=\frac{\partial H^{\prime}}{\partial \phi^{\prime}}=\epsilon \sum_{\nu \in M} i G_{\nu}\left(\mathbf{I}^{\prime}, \epsilon\right) e^{i \nu \cdot \phi^{\prime}} \nu .
$$

Ou seja, $\dot{\mathbf{I}}$ é uma combinação linear dos vetores $\nu$, logo o movimento de $\mathbf{I}^{\prime}$ está sobre um plano paralelo a $M$ passando pelo ponto $\mathbf{I}^{\prime}(0)$, que chamaremos de $\Pi_{M}\left(\mathbf{I}^{\prime}(0)\right)$,

Afirmação 2. A hipótese da quase convexidade nos diz que:

- (a) O plano $\Pi_{M}$ é transversal a superfície ressonante $\Sigma_{M}$

- (b) O Hamiltoniano não perturbado $h$, restrito ao plano $\Pi_{M}$, tem um máximo ou minimo, no ponto $\tilde{\mathbf{I}} \in \Pi_{M} \cap \Sigma_{M}$.

\section{Demonstração.}

(a) Suponha que existe um vetor $\mathbf{v} \in \Pi_{M}(\mathbf{I}(0))$,

$$
\mathbf{v}=\sum_{\nu \in M} c_{\nu} \nu
$$

tangente a $\Sigma_{M}$ em algum ponto $\tilde{\mathbf{I}} \in \Sigma_{M}$. Como $\omega(\mathbf{I}) \cdot \mathbf{v}=0$ em $\Sigma_{M}$, então $h^{\prime \prime}(\mathbf{I}) \mathbf{v}=\frac{\partial}{\partial I} \omega(\mathbf{I}) \cdot \mathbf{v}$ é ortogonal a $\Sigma_{M}$ para $\mathbf{I} \in \Sigma_{M}$, assim $h^{\prime \prime}(\tilde{\mathbf{I}}) \mathbf{v} \cdot \mathbf{v}=0$. Observe que, de acordo com a equação (A.25), também temos $\omega(\tilde{\mathbf{I}}) \cdot \mathbf{v}=0$. O que contraria a hipótese da quase convexidade.

(b) De fato, expandindo temos

$$
h(\tilde{\mathbf{I}}+\mathbf{v})=h(\tilde{\mathbf{I}})+\omega(\tilde{\mathbf{I}}) \cdot \mathbf{v}+\frac{1}{2} h^{\prime \prime}(\tilde{\mathbf{I}}) \mathbf{v} \cdot \mathbf{v}+\cdots
$$

Para $\mathbf{v} \in \Pi_{M}$ o segundo termo da expansão é igual a zero e o terceiro termo tem sinal definido, pela hipótese da quase convexidade. Digamos $\frac{1}{2} h^{\prime \prime}(\tilde{\mathbf{I}}) \mathbf{v} \cdot \mathbf{v}$ seja positivo, então $h(\tilde{\mathbf{I}}+\mathbf{v}) \geq h(\tilde{\mathbf{I}})$, logo $h$ tem um mínimo em $\tilde{\mathbf{I}}$. 


\section{A.7 A Conservação da Energia}

Como uma consequência das afirmações acima a conservação da energia garante o confinamento das ações. De fato, $h$ possuindo um extremo, então as superfícies de nível tem a forma de elipses concêntricas em torno de $\tilde{\mathbf{I}}$ sobre o plano $\Pi_{M}$. É importante ressaltar que pela proposição 2, a dinâmica do sistema Hamiltoniano (A.15), para tempos exponencialmente longos, é descrita em $U_{M}$ pelo Hamiltoniano

$$
h\left(\mathbf{I}^{\prime}\right)+\epsilon G\left(\mathbf{I}^{\prime}, \phi^{\prime}, \epsilon\right) .
$$

Assim, se a energia é conservada, $h$ tem uma oscilação limitada por $\epsilon$ durante o movimento. Além disso, o movimento das ações está confinado entre duas superfícies de nível próximas. 


\section{Referências Bibliográficas}

Arnold V., Instability of dynamical systems with several degrees of freedom., Sov. Math, 1964, vol. 5, p. 581-585

Beaugé C., Michtchenko T. A., Ferraz-Mello S., Planetary migration and extrasolar planets in the 2/1 mean-motion resonance, MNRAS, 2006, vol. 365, p. 1160

Cachucho F., Cincotta P. M., Ferraz-Mello S., Chirikov diffusion in the asteroidal threebody resonance (5, -2, -2), Celestial Mechanics and Dynamical Astronomy, 2010, vol. 108, p. 35

Cachucho da Silva, F. Difusão Lenta: Teorias e Aplicação À Ressonância 5-2-2 da Família de (490) veritas, São Paulo: Universidade de São Paulo, 2003, Tese de Doutorado, 174 p.

Chandrasekhar S., Stochastic Problems in Physics and Astronomy, Rev. Mod. Phys., 1943, vol. 15 , p. 1

Chirikov B. V., A universal instability of many-dimensional oscillator systems, Physics Reports, 1979, vol. 52, p. 263

Cincotta P. M., Arnold diffusion: an overview through dynamical astronomy, New Astronomy Reviews, 2002, vol. 46, p. 13

Ellis K. M., Murray C. D., The Disturbing Function in Solar System Dynamics, Icarus, 2000, vol. 147, p. 129

Fernandez S., Beauge C., Asteroids in the 1:3:2 commensurability. In IAU Colloq. 96: The Few Body Problem , vol. 140 of Astrophysics and Space Science Library, 1988, p. 131 
Ferraz-Mello S., A Symplectic Mapping Approach to the Study of the Stochasticity in Asteroidal Resonances, Celestial Mechanics and Dynamical Astronomy, 1996, vol. 65, p. 421

Ferraz-Mello S., Canonical Perturbation Theories: Degenerate Systems and Resonance. Springer, New York, 2007

Ferraz-Mello S., Beaugé C., Michtchenko T. A., Evolution of Migrating Planet Pairs in Resonance, Celestial Mechanics and Dynamical Astronomy, 2003, vol. 87, p. 99

Ferraz-Mello S., Klafke J. C., A model for the study of very-high-eccentricity asteroidal motion: the 3:1 resonance.. In Predictability, Stability, and Chaos in N-Body Dynamical Systems , 1991, p. 177

Gregory P. C., Bayesian exoplanet tests of a new method for MCMC sampling in highly correlated model parameter spaces, MNRAS, 2011, vol. 410, p. 94

Hadjidemetriou J. D., Asteroid motion near the 3:1 resonance, Celestial Mechanics and Dynamical Astronomy, 1993, vol. 56, p. 563

Hadjidemetriou J. D., Voyatzis G., The 1/1 resonance in extrasolar systems. Migration from planetary to satellite orbits, Celestial Mechanics and Dynamical Astronomy, 2011, vol. 111, p. 179

Hughes S., The Computations Of Tables Of Hansen Coefficients, Celestial Mechanics and Dynamical Astronomy, 1981, vol. 25, p. 101

Klafke J. C., Ferraz-Mello S., Michtchenko T., Very-High Librations at Some Higher-Order Resonances. In Chaos, Resonance, and Collective Dynamical Phenomena in the Solar System , vol. 152 of IAU Symposium, 1992, p. 153

Lega E., Guzzo M., Froeschlé C., A numerical study of the hyperbolic manifolds in a priori unstable systems. A comparison with Melnikov approximations, Celestial Mechanics and Dynamical Astronomy, 2010, vol. 107, p. 115

Lega E., Guzzo M., Froeschlé C., Detection of Arnold diffusion in Hamiltonian systems, Physica D: Nonlinear Phenomena, 2003, vol. 182, p. 179 
Lichtenberg A. J., Lieberman M. A., Regular and Chaotic Dynamics 2nd edn. No. 38 in Applied Mathematical Sciences, Springer-Verlag New York, NY, 1992

Mayor M., Bonfils X., Forveille T., Delfosse X., Udry S., Bertaux J.-L., Beust H., Bouchy F., Lovis C., Pepe F., Perrier C., Queloz D., Santos N. C., The HARPS search for southern extra-solar planets. XVIII. An Earth-mass planet in the GJ 581 planetary system, A\&A, 2009, vol. 507, p. 487

Michtchenko T., Beaugé C., Ferraz-Mello S., Stationary Orbits in Resonant Extrasolar Planetary Systems, Celestial Mechanics and Dynamical Astronomy, 2006, vol. 94, p. 411

Michtchenko T. A., Ferraz-Mello S., Resonant Structure of the Outer Solar System in the Neighborhood of the Planets, AJ, 2001, vol. 122, p. 474

Murray N., Holman M., Potter M., On the Origin of Chaos in the Asteroid Belt, AJ, 1998, vol. 116 , p. 2583

Nesvorný D., Morbidelli A., An Analytic Model of Three-Body Mean Motion Resonances, Celestial Mechanics and Dynamical Astronomy, 1998a, vol. 71, p. 243

Nesvorný D., Morbidelli A., Three-Body Mean Motion Resonances and the Chaotic Structure of the Asteroid Belt, AJ, 1998b, vol. 116, p. 3029

Roig F., Ferraz-Mello S., A symplectic mapping approach of the dynamics of the Hecuba gap, Planet. Space Sci., 1999, vol. 47, p. 653

S. Udry X. Bonfils X. Delfosse T. Forveille M. Mayor C. Perrier F. Bouchy C. Lovis F. Pepe D. Queloz J.-L. Bertaux The HARPS search for southern extra-solar planets, A\&A, 2007, vol. 469, p. L43

Tadeu dos Santos M., Silva G. G., Ferraz-Mello S., Michtchenko T. A., A new analysis of the GJ581 extrasolar planetary system, Celestial Mechanics and Dynamical Astronomy, 2012, vol. 113, p. 49

Tomé T. e Oliveira M., Dinâmica Estocástica e Irreversibilidade 1nd edn. No. 35 in Acadêmica, EDUSP São Paulo, SP, 2001 
Varvoglis H., Chaos, random walks and diffusion in Hamiltonian systems. In: Benest, D. e Lega, E.(eds), Hamiltonian systems and Fourier analysis, new prospects for gravitational dynamics. 1 nd. Nice: Cambridge Scientific Publishers, 2005, p. 247

Varvoglis H., Tsiganis K., Hadjidemetriou J., Stable Chaos and Kirkwood Gaps. In 5th Hellenic Astronomical Conference , 2001

Vogt S. S., Butler R. P., Haghighipour N., GJ 581 update: Additional evidence for a Super-Earth in the habitable zone, Astronomische Nachrichten, 2012, vol. 333, p. 561

Vogt S. S., Butler R. P., Rivera E. J., Haghighipour N., Henry G. W., Williamson M. H., The Lick-Carnegie Exoplanet Survey: A 3.1 Planet in the Habitable Zone of the Nearby M3V Star Gliese 581, ApJ, 2010, vol. 723, p. 954

Wisdom J., The origin of the Kirkwood gaps - A mapping for asteroidal motion near the 3/1 commensurability, AJ, 1982, vol. 87, p. 577

Wisdom J., Chaotic behavior and the origin of the 31 Kirkwood gap, Icarus, 1983, vol. 56, p. 51 